\title{
A Conceptual Design Methodology for Predicting the Aerodynamics of Upper Surface Blowing on Airfoils and Wings
}

\author{
by: \\ Ernest B. Keen \\ Thesis submitted to the faculty of \\ Virginia Polytechnic Institute \& State University \\ in partial fulfillment of the requirements for the degree of \\ Master of Science \\ in \\ Aerospace Engineering
}

APPROVED:

William H. Mason, Committee Chairman

\begin{tabular}{l}
\hline Joseph A. Schetz \\
\hline
\end{tabular}

Paul Gelhausen

November, 2004

Blacksburg, Virginia

Keywords: Upper Surface Blowing, USB, aerodynamics, conceptual design, jet-flap 


\title{
A Conceptual Design Methodology for Predicting the Aerodynamics of Upper Surface Blowing on Airfoils and Wings
}

by:

\author{
Ernest B. Keen
}

\begin{abstract}
(ABSTRACT)
One of the most promising powered-lift concepts is Upper Surface Blowing (USB), where the engines are placed above the wing and the engine exhaust jet becomes attached to the upper surface. The jet thrust can then be vectored by use of the trailing edge curvature since the jet flow tends to remain attached by the "Coanda Effect". Wind tunnel and flight-testing have shown USB aircraft to be capable of producing maximum lift coefficients near 10. They have the additional benefit of shielding the engine noise above the wing and away from the ground.

Given the potential gains from USB aircraft, one would expect that conceptual design methods exist for their development. This is not the case however. While relatively complex solutions are available, there is currently no adequate low-fidelity methodology for the conceptual and preliminary design of USB or USB/distributed propulsion aircraft. The focus of the current work is to provide such a methodology for conceptual design of USB aircraft. Based on limited experimental data, the new methodology is shown to compare well with wind tunnel data.

In this thesis we have described the new approach, correlated it with available 2-D data, and presented comparisons of our predictions with published USB data and an existing non-linear vortex lattice method. The current approach has been shown to produce good results over a broad range of propulsion system parameters, wing geometries, and flap deflections. In addition, the semi-analytical nature of the methodology will lend itself well to aircraft design programs/optimizers such as ACSYNT. These factors make the current method a useful tool for the design of USB and USB/distributed propulsion aircraft.
\end{abstract}




\section{Acknowledgments}

I would like first to thank my Lord, Jesus Christ, with whom everything is possible, and without whom I am nothing.

Also, I am more than grateful for the guidance and advise of my advisor, William Mason. His ideas and experience have saved me on many occasions, and he has taught me more about practical engineering than anyone I've been around. It has been a pleasure to be associated with him and his students.

To my family, Jack, Sheila, Jordan, I owe my thanks for years of love, support, open arms, and open ears. Their belief in me has pushed me to this point, and will drive me in the years to come.

In addition, I would like to thank my friends at AVID, LLC for their funding and support of this research, and the friendships I can't quantify. They have given me opportunities where there seemed to be none. I hope to represent them and their commitment to excellence well with this work.

Lastly, I would like to thank my friends, here at Virginia Tech, back home, and out in the wide,wide world. You've kept me sane, you've kept me laughing, and you've kept me going.... and this is just the beginning. 


\section{Table of Contents}

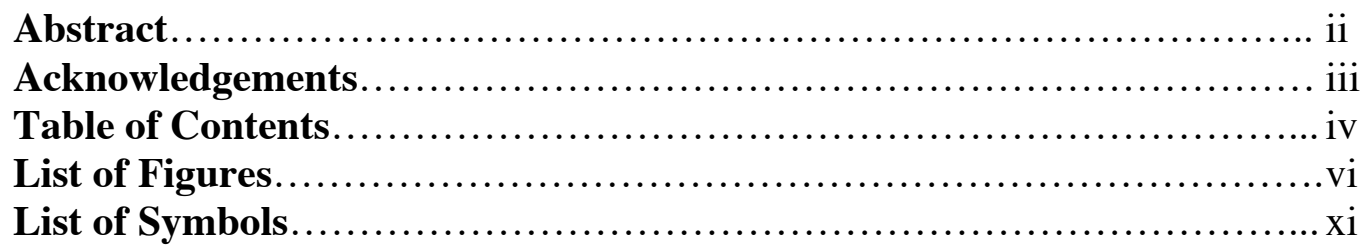

Chapter 1, Introduction......................................................... 1

Chapter 2, Physics of Upper Surface Blowing................................. 5

2.1 Basic flow around an airfoil with no blowing.................... 6

2.2 Effects of trailing edge blowing................................. 7

2.3 Effects of surface blowing .................................. 14

2.4 Breakdown of forces......................................... 17

2.5 Adverse effects................................................. 19

2.6 Three-Dimensional behavior.................................. 21

Chapter 3, Thrust/Drag Bookkeeping for USB Designs...................... 23

3.1 Losses and bookkeeping.................................... 24

3.2 Estimating losses............................................. 26

Chapter 4, Development of a 2-D analytical method........................... 32

4.1 Basis for method/overview.................................... 32

4.2 Spence's jet-flap theory......................................... 34

4.2.1 Lift Coefficient.................................... 34

4.2.2 Pitching moment coefficient........................ 37

4.3 Circular Streamline Theory ................................ 38

4.3.1 Derivation of CST for USB .......................... 40

4.3.2 Lift, Drag, and Pitching moment calculations............. 47

4.4 Drag estimation............................................... 49

4.5 Entrainment Effects............................................ 50

4.6 Total 2-D equations........................................... 51

4.6.1 Lift Coefficient.......................................... 51

4.6.2 Pitching Moment Coefficient........................... 52

4.6.3 Drag Coefficient................................... 52 
Chapter 5, Correlation of 2-D method with Experimental Data................. 53

5.1 Description of Published Experiments...............................53

5.1 .1 History and Overview..................................53

5.1.2 Experimental data...................................... 56

5.2 Empirical factors and their physical basis......................... 58

5.2.1 The "n" factor............................................ 59

5.2.2 The " $\eta_{\text {ent }}$ " factor............................................. 60

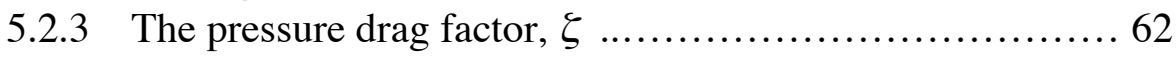

5.3 Presentation of 2-D correlation................................ 63

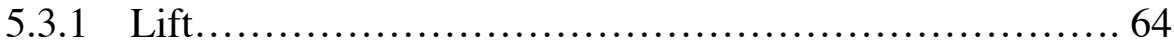

5.3.2 Pitching Moment.......................................67

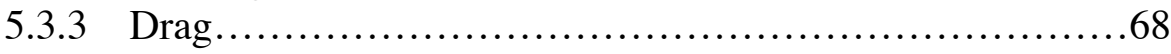

Chapter 6, Three-Dimensional USB Performance Prediction Method ......... 72

6.1 Overview of the Weissinger method.............................72

6.2 Using the new 2-D USB method with the Weissinger method ...... 75

6.2.1 The 2-D USB estimations.................................. 75

6.2.2 The High-Lift module.................................. 77

6.3 Limitations of method........................................ 79

6.3.1 Spanload Oscillation..................................... 80

6.3.2 Stall prediction........................................ 84

6.3.3 Nacelle and Fuselage effects.............................. 85

6.4 Lateral-Directional Characteristics.............................. 86

Chapter 7, Comparison with 3-D experimental results.......................... 88

7.1 NASA Experimental USB Reports............................ 89

7.1.1 Case 1: Small-scale, four-engine config. (TN 8061)......... 89

7.1.2 Case 2: Large-scale, twin-engine config. (TN 7526)........ 95

7.1.3 Case 3: Small-scale, four-engine config. (TN 7399)........ 104

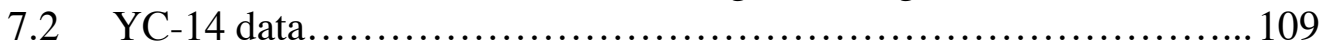

7.3 Brief Comparison with a Non-Linear VLM....................... 114

Chapter 8, Conclusions/recommendations...................................... 120

References................................................................122

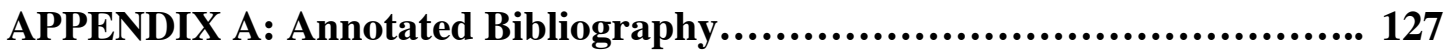

APPENDIX B: Sample of Data Generation for 2-D Calculations................. 142

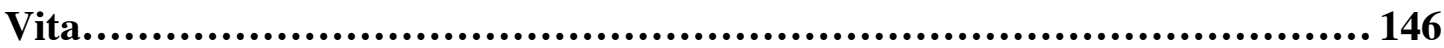




\section{List of Figures}

Figure Page

1-1 Examples of Research and Experimental USB aircraft................ 2

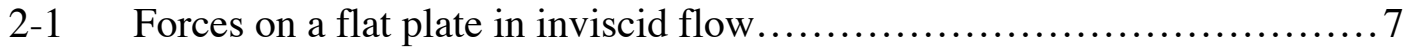

2-2 Sketch of blown section with control surfaces........................ 9

2-3 Pressure distribution on a jet-flapped airfoil from ref. $16 \ldots \ldots \ldots \ldots \ldots \ldots \ldots 12$

2-4 Sketch of the trailing edge of a jet-flapped airfoil...................... 12

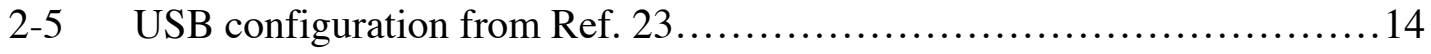

2-6 YC-14 USB nacelle/nozzle/flap system........................... 16

2-7 Sketch of forces for an idealized USB configuration.................. 18

3-1 Thrust-plus-drag polars for a four-engine USB configuration at $35 \mathrm{deg}$ flap deflection......................................... 26

3-2 Sketch of nozzle exhaust flow angles............................ 28

3-3 Comparison of experimental flow turning angle with empirical relation......................................................... 29

3-4 Typical turning efficiency range for USB flap systems................... 30

4-1 Schematic representation of the current method....................... 33

4-2 Agreement of $\mathrm{d} C_{L} / \mathrm{d} \delta_{f}$ using derived approximate function............... 36

4-3 Example of the velocity profile of a wall jet flowing over a convex surface with an external stream................................... 39

4-4 Streamlines and velocity profile produced by a point vortex............. 40

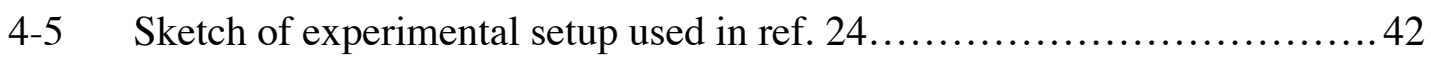

4-6 Comparison of CST with experimental data from ref $23 \ldots \ldots \ldots \ldots \ldots \ldots . \ldots 2$

4-7 Sketch of actual and composite velocity profiles for a wall jet............. 43

4-8 Comparison of CST with experimental data from ref $24 \ldots \ldots \ldots \ldots \ldots \ldots \ldots . \ldots 6$ 
4-9 Comparison of CST with static ground-test data from ref $8 \ldots \ldots \ldots \ldots \ldots . \ldots 46$

4-10 Comparison of CST with flight test data from ref $8 \ldots \ldots \ldots \ldots \ldots \ldots \ldots \ldots . \ldots \ldots$

4-11 Drawing of a USB surface discretized into small, linear segments......... 48

5-1 Propulsive wing section used in experiments of ref $44 \ldots \ldots \ldots \ldots \ldots \ldots \ldots \ldots$

5-2 Sample of $C_{l}$ vs. blowing coefficient from experiment....................57

5-3 Sample of $C_{m, c / 4}$ vs. $C_{l}$ from experiment............................ 57

5-4 Experimental data for pressure drag vs. blowing coefficient.............. 58

5-5 Pressure coefficients due to blowing effects........................ 60

5-6 2-D comparison of current method, Jet-flap theory, and experiment for $\alpha=-5$ degrees.............................................

5-7 2-D comparison of current method, Jet-flap theory, and experiment for $\alpha=0$ degrees.............................................. 65

5-8 2-D comparison of current method, Jet-flap theory, and experiment

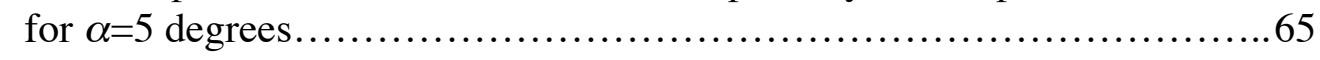

5-9 2-D comparison of current method, Jet-flap theory, and experiment for $\alpha=10$ degrees................................................. 66

5-10 2-D comparison of current method, Jet-flap theory, and experiment

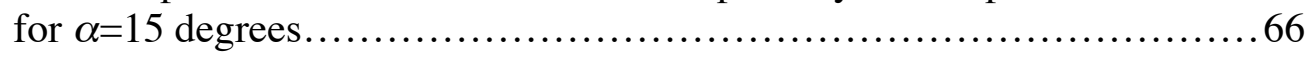

5-11 Comparison of $C_{m, c / 4}$ for current method predictions and experiment.......67

5-12 Comparison of $C_{m, c / 4}$ for current method predictions and experiment........68

5-13 Correlation of pressure drag predictions for $\alpha=0$ degrees................69

5-14 Correlation of pressure drag predictions for $\alpha=5$ degrees...............69

5-15 Correlation of pressure drag predictions for $\alpha=10$ degrees................ 70

5-16 Correlation of pressure drag predictions for $\alpha=15$ degrees...............70

6-1 Vortex system representation of a lifting surface $\ldots \ldots \ldots \ldots \ldots \ldots \ldots \ldots \ldots \ldots$ 
6-2 Sample output data from MATLAB 2-D USB code.................... 78

6-3 Planform view of wing used in experiments of ref $8 \ldots \ldots \ldots \ldots \ldots \ldots \ldots \ldots 1$

6-4 Effective angle of attack across wing semi-span at $\alpha=20 \mathrm{deg} . \ldots \ldots \ldots \ldots . . . .81$

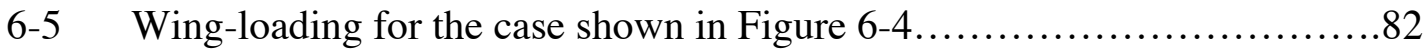

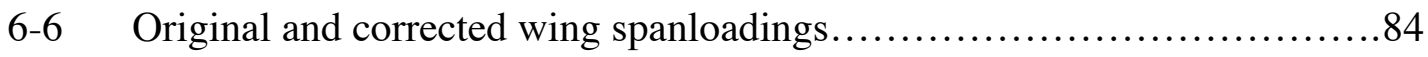

6-7 Sectional data regions across a wing for an engine-out case $\ldots \ldots \ldots \ldots \ldots . \ldots 6$

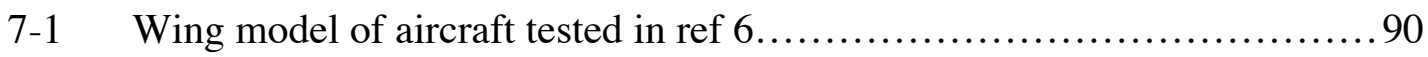

7-2 $C_{L}$ variation with alpha for 0 deg flaps at 2 thrust settings................ 91

7-3 Pitching moment vs. alpha for 0 deg flaps at 2 thrust settings............ 92

7-4 Thrust-plus-drag polar for $C_{T}=0.9,0$ deg flaps $\ldots \ldots \ldots \ldots \ldots \ldots \ldots \ldots \ldots . \ldots 3$

7-5 Thrust-plus-drag polar for $C_{T}=2.0,0$ deg flaps $\ldots \ldots \ldots \ldots \ldots \ldots \ldots \ldots \ldots \ldots$

7-6 $C_{L}$ variation with alpha for $35 \mathrm{deg}$ USB flaps at 3 thrust settings........... 94

7-7 Pitching moment about the C.G. for 35 deg USB flaps at 3 thrust settings................................................ 94

7-8 Thrust-plus-drag polars for $35 \mathrm{deg}$ USB flaps at 3 thrust settings.......... 95

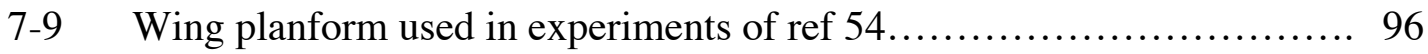

7-10 $C_{L}$ variation with alpha for 0 deg flaps at 2 thrust settings............... 97

7-11 $C_{L}$ variation with alpha for 20 deg flaps at 2 thrust settings............... 97

7-12 $C_{L}$ variation with alpha for 40 deg flaps at 2 thrust settings............... 98

7-13 $C_{L}$ variation with alpha for 60 deg flaps at 2 thrust settings............... 98

7-14 Example of induced drag correction for thrust-plus-drag polars............ 100

7-15 Thrust-plus-drag polars for 2 power settings at 0 deg flaps.............. 100

7-16 Thrust-plus-drag polars for 2 power settings at 20 deg flaps............... 101

7-17 Thrust-plus-drag polars for 2 power settings at 40 deg flaps............... 101 
7-18 Thrust-plus-drag polars for 2 power settings at 60 deg flaps...............102

7-19 Pitching moment about the aircraft C.G. vs. alpha for 0 deg flaps at 2 thrust settings 103

7-20 Pitching moment about the aircraft C.G. vs. alpha for 20 deg flaps at 2 thrust settings 103

7-21 Pitching moment about the aircraft C.G. vs. alpha for $40 \mathrm{deg}$ flaps at 2 thrust settings................................................ 104

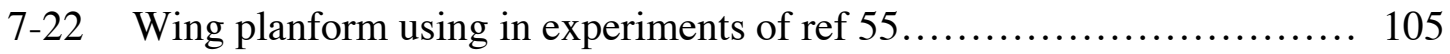

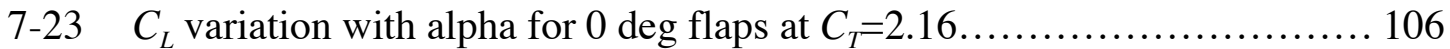

7-24 Pitching moment about the aircraft C.G. vs. alpha for 0 deg flaps

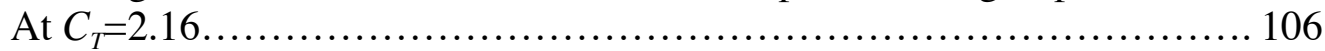

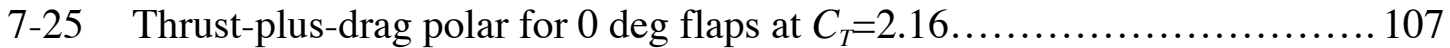

7-26 $C_{L}$ variation with alpha for $35 \mathrm{deg}$ flaps at 2 thrust settings............... 108

7-27 Thrust-plus-drag polars for 35 deg flaps at 2 thrust settings.............. 108

7-28 Pitching moment about the aircraft C.G. vs. alpha for $35 \mathrm{deg}$ flaps

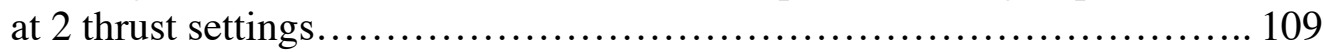

7-29 Wing planform for the Boeing YC-14.......................... 110

7-30 Upper surface profiles for various flap deflections.....................111

7-31 $C_{L}$ variation with alpha for takeoff configuration at 2 thrust settings....... 112

7-32 Thrust-plus-drag polars for takeoff configuration at 2 thrust settings........ 112

7-33 $C_{L}$ variation with alpha for landing configuration.................... 113

7-34 Thrust-plus-drag polar for landing configuration........................ 114

7-35 $C_{L}$ predictions from VLM and current method for 45 deg deflected radius flap at 2 thrust settings 
7-36 Pitching moments about the C.G. for 45 deg flaps at 2 thrust settings...... 117

7-37 Thrust-plus-drag polars for 45 deg flaps at 2 thrust settings.............. 117

7-38 Comparison of non-linear VLM to CST surface pressures ................ 118 


\section{List of Symbols}

$b \quad-\quad$ wing span (ft)

$b / 2 \quad-\quad$ semi-span (ft)

$b_{U S B} \quad-\quad$ span of upper surface blowing (ft)

c - local chord of airfoil (ft)

$c_{f} \quad-\quad$ chord of trailing edge flap (ft)

$C_{j}, C_{\mu}-\quad$ sectional blowing coefficient $/$ momentum flux coefficient $=\frac{\dot{m} V_{e x i t}}{q c b_{U S B}}$

$C_{l} \quad-\quad$ sectional lift coefficient

$C_{L} \quad-\quad$ wing lift coefficient

$C_{L \alpha} \quad$ - lift curve slope

$C_{d} \quad-\quad$ sectional drag coefficient

$C_{d p} \quad-\quad$ sectional pressure drag coefficient

$C_{D i} \quad$ - $\quad$ induced drag coefficient

$C_{D} \quad-\quad$ total drag coefficient

$C_{m} \quad-\quad$ sectional pitching moment coefficient, about quarter-chord

$C_{M, c g} \quad$ - wing pitching moment coefficient about aircraft C.G. location

$C_{T} \quad-\quad$ total thrust coefficient of aircraft $=\frac{\text { Total Thrust }}{q S}$

CG - center of gravity of aircraft

$e \quad-\quad$ span efficiency factor

$E \quad-\quad$ ratio of flap chord to local airfoil chord $=\frac{c_{f}}{c}$

$h \quad$ - $\quad$ height of nozzle exit (ft)

$\dot{\mathbf{m}} \quad$ - $\quad$ engine mass flow rate (slug/s)

$p_{\infty} \quad-\quad$ freestream static pressure $\left(\mathrm{lb} / \mathrm{ft}^{2}\right)$

$p \quad$ - local static pressure $\left(\mathrm{lb} / \mathrm{ft}^{2}\right)$

$q_{\infty} \quad$ - $\quad$ freestream dynamic pressure $\left(\mathrm{lb} / \mathrm{ft}^{2}\right)$

$q_{j e t} \quad$ - dynamic pressure of jet $\left(\mathrm{lb} / \mathrm{ft}^{2}\right)$

$\mathbf{q}_{\mathrm{i}} \quad$ - $\quad$ 2-D vector from airfoil quarter chord to midpoint of $\mathrm{i}^{\text {th }}$ panel

$R \quad$ - $\quad$ radius of curvature $(\mathrm{ft})$

$R_{\text {ref }} \quad$ - reference radial location (ft) 


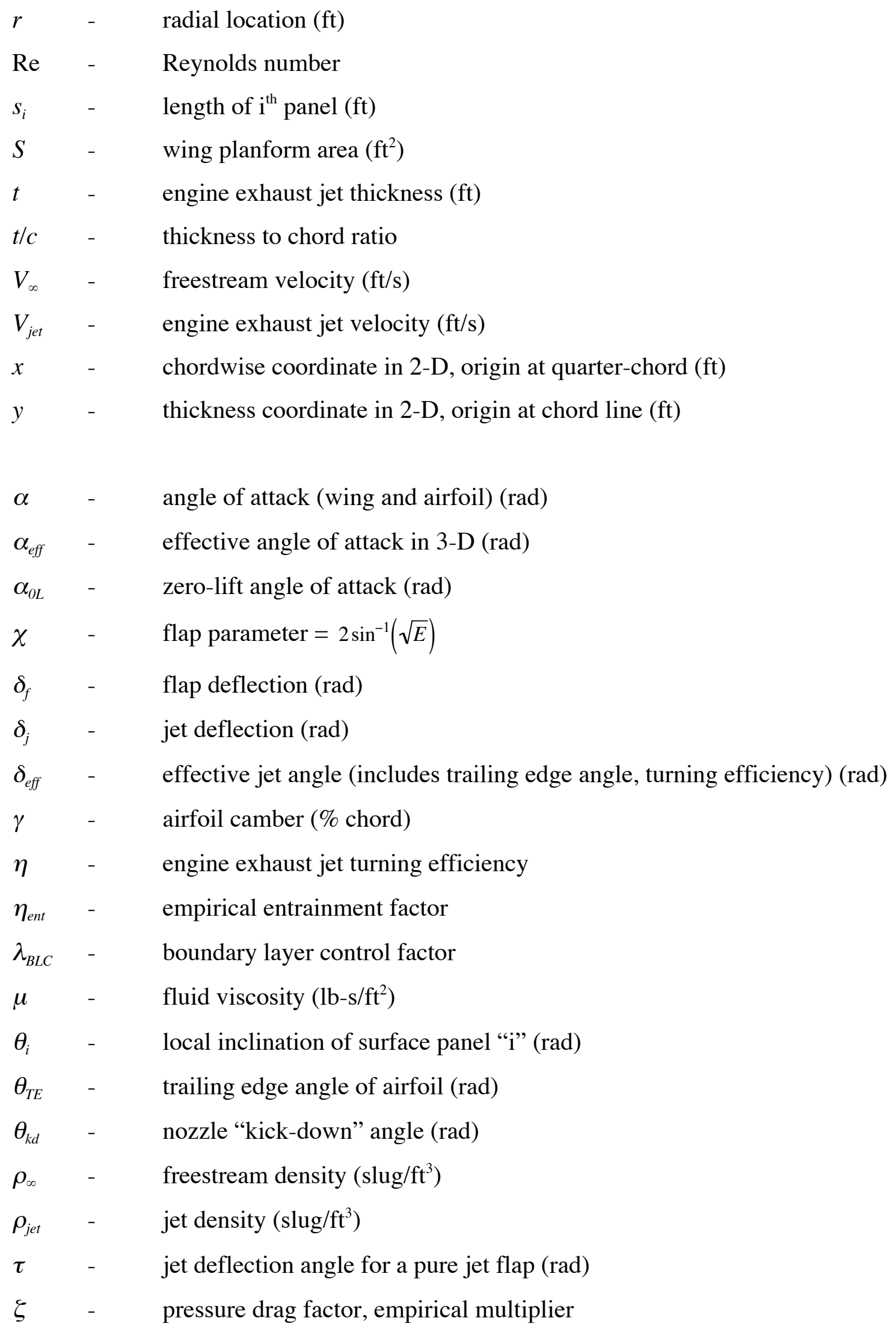




\section{Chapter 1: Introduction}

As modern aircraft have grown in size and complexity to become more cost efficient for operators, so too has the infrastructure needed to accommodate them. Programs such as the Airbus A380, which will carry over 800 passengers but only operate out of 60 airports worldwide ${ }^{(1)}$, will do little to relieve this trend. This has placed huge demands on the current "hub-and-spoke" airport system. With a limited throughput on major runways, huge delays are often experienced. Such was the recent case at Chicago's O'Hare airport where delays reached critical limits, reducing the on-time arrivals in the whole air system by $15 \%{ }^{(2)}$. Hundreds of millions of dollars are lost due to air traffic delays while adjacent "stub" runways go unused and smaller regional airports operate at small fractions of their capacity.

Why is the air system consistently pushed to its limits? Because there are no aircraft in service that can meet the performance demands associated with short-field operations. Clearly, there is a need for powered-lift aircraft that can demonstrate exceptional takeoff and landing performance. While the need for Short Takeoff and Landing (STOL) is 
becoming apparent in civil aviation, it is already becoming a mode of operation for military branches such as the Navy and Marines. In addition, programs such as NASA's Small Aircraft Transportation System (SATS) ${ }^{(3)}$ and Runway Independent Aircraft $(\mathrm{RIA})^{(4)}$ are implicitly dependent on the development of aircraft with breakthrough highlift performance.

One such powered-lift option is Upper Surface Blowing (USB), where the engines are placed above the wing and the exhaust jet becomes attached to the upper surface. The jet can then be vectored by use of trailing edge curvature since the jet tends to remain attached to the surface by the "Coanda Effect". Wind tunnel and flight testing have shown USB aircraft to be capable of producing maximum lift coefficients near $10^{(5),(6),(7)}$. They have the additional benefit of shielding the engine noise above the wing and away from the ground ${ }^{(8)}$. This technology has been successfully demonstrated in the past on experimental and research aircraft. Figure 1-1 shows some examples. ${ }^{(9),(10)}$

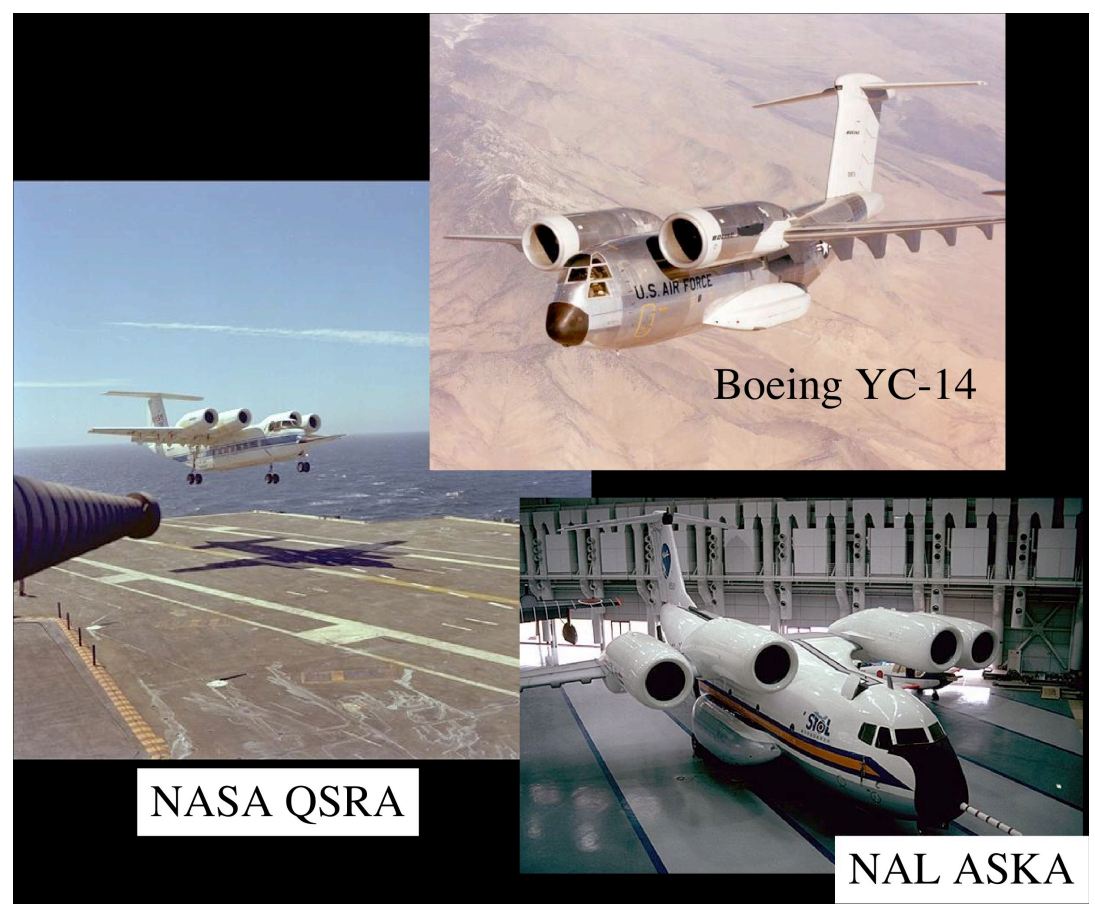

Figure 1-1: Examples of Research and Experimental USB aircraft 
Given the potential performance gains from USB aircraft, one would expect that conceptual design methods exist for their development. However, this is not the case. There is currently no low-fidelity methodology for the conceptual and preliminary design of USB or USB/distributed propulsion aircraft. This can discourage a conceptual design from making the transition to powered-lift. It has been stated that " $90 \%$ of the cost of a product is committed during the first $10 \%$ of the design cycle". ${ }^{(11)}$ It is easy to see the importance of providing good estimations of performance at early design stages. Given the inherent sensitivity of a USB design to high-lift performance, good estimations can mean big savings in the design process.

The current state-of-the-art for 3-D USB predictions are zonal methods based on coupling the Navier-Stokes equations with a potential flow panel method ${ }^{(12)}$. However, this is a complicated, high-fidelity model and cannot reasonably be implemented until the detail design phase. Low-fidelity models have changed very little from those established in the 1970s. Inviscid solutions using panel methods can be quite useful but require more geometry detail, typically require an iterative solution for the jet boundaries, and cannot predict drag. The most successful panel method was produced by Mendenhall ${ }^{(13)}$ using a non-linear vortex lattice formulation. A comparison of this method to the proposed methodology is presented in this work. The classic "Jet-Flap" theory given by Spence ${ }^{(14)}$ is easy to use but, as will be shown in the chapters that follow, is inadequate for USB predictions.

The purpose of the current work is to provide a low-fidelity performance prediction methodology for Upper Surface Blown wings suited to use in the conceptual design phase. This methodology will be demonstrated to have appropriate accuracy for early 
design phases. It is also fast, easy to use, and robust enough to handle nearly all USB configurations. Its ease of use will allow it to be integrated readily into existing aircraft design programs. Table 1-1 shows a comparison of various methods for USB performance calculations.

Table 1-1: Comparison of USB prediction methods

\begin{tabular}{|c|c|c|c|c|}
\hline & Low-fidelity? & Accurate? & Fast? & $\begin{array}{c}\text { Easy to Use / Easy to } \\
\text { integrate? }\end{array}$ \\
\hline $\begin{array}{c}\text { Coupled N-S / } \\
\text { potential flow }\end{array}$ & & & & \\
\hline Panel methods & & & & \\
\hline Jet-Flap theory & & & & \\
\hline Proposed method & & & & \\
\hline
\end{tabular}

The purpose of this work is to introduce a new approach for analyzing the problem, to correlate it with 2-D data, and then extend it to 3-D performance predictions. The focus is directed towards providing the methodology for low-fidelity USB performance predictions. In addition, this work contains a description of a thrust-drag bookkeeping method for USB designs. Also included is an annotated bibliography of a large number of papers on the subject. The work contained in this thesis will be valuable for engineers pursuing design concepts incorporating USB and USB/distributed-propulsion. 


\section{Chapter 2: Physics of Upper Surface Blowing}

The changes induced in a flowfield when blowing is introduced can be dramatic. The various effects have been documented to different degrees by previous researchers. However, a broad, qualitative explanation of the total process has yet to be given. This is due primarily to two things. First, there is a relatively small base of experimental data upon which to draw conclusions. This is further complicated by the fact that most experimental data is "configuration dependent", meaning that generally a specific aircraft configuration was tested. Thus, the basic physics can sometimes become obscured. Second, this area of research hasn't received much attention for some time.

It is the goal of this chapter to examine the effects of blowing on the flowfield around an airfoil, with our particular interest being upper surface blowing. Some of the phenomena involved are well understood, while others are not. In the latter cases, possible explanations are offered with their basis in aerodynamic theory and published experimental data. 


\section{1 - Basic Flow around an Airfoil with no blowing}

Consider an airfoil immersed in an inviscid, uniform, steady flow. The body will generate a circulation value specified by the enforcement of the Kutta condition at the trailing edge. The Kutta-Joukowski theorem:

$$
L=\rho U \Gamma
$$

says that the lift is uniquely determined by the freestream conditions and the circulation. Therefore, the greater the circulation, the greater the lift generated. The appropriate value for the circulation is determined by the Kutta condition, since potential flow gives an infinite number of possible solutions. The Kutta condition simply states that at a finite angle trailing edge there must be a stagnation point, or for a cusped trailing edge, the upper and lower velocities must be parallel and equal.

Equation (2-1) requires that the lift force be perpendicular to the freestream regardless of the orientation of the airfoil. Thus, the drag for inviscid analysis must be zero. This phenomenon is explained by Mason ${ }^{(15)}$ as being a result of the leading-edge suction force. This force is a result of the rapid expansion the flow experiences around the leading edge. It becomes more pronounced as the stagnation point moves and it produces a forward force parallel to the chord line. Consider Figure 2-1 from $\operatorname{Mason}^{(15)}$ showing a flat plate airfoil at a given angle of attack in an inviscid, attached flow. 


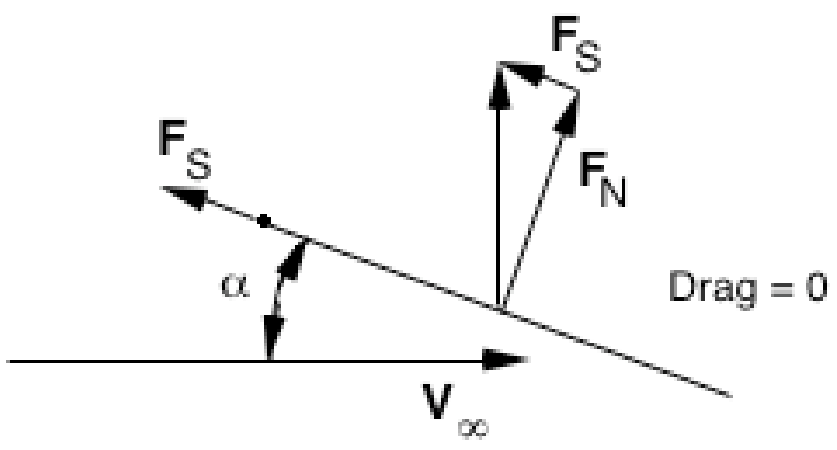

Figure 2-1: Forces on a flat plate in inviscid flow

Thus, any component of the lift (which acts normal to $\mathbf{V}_{\infty}$ ) parallel to the chord-line must be equal and opposite the leading edge suction force.

The addition of leading or trailing edge flaps will increase an airfoil's lift performance. Trailing edge flaps change the effective camber of the airfoil. The circulation is thus increased, and extra lift is gained. Leading edge flaps (or slats) primarily affect the maximum lift that can be achieved. These devices "soften" the rapid leading edge expansion that is present when an airfoil is operating at high circulation. In so doing, they allow the airfoil to go to higher angles of attack before stall. These devices, and the art of obtaining high-lift from mechanical devices, are explained thoroughly by A.M.O. Smith ${ }^{(16)}$.

\section{2 - Effects of Trailing Edge Blowing}

The benefits of introducing a high-energy jet somewhere in a wing flowfield have been recognized for some time. Smith ${ }^{(16)}$ traces the use of boundary layer control via surface blowing to 1913 . Korbacher ${ }^{(17)}$ says that the influence of the propeller slipstream on aircraft wings and control surfaces was documented by 1935 . The advent of the jet engine, with very high-energy exhaust flow and a ready supply of high-pressure air, 
opened a world of possibilities for powered lift. Methods for analyzing jet-flapped airfoils were developed by Spence ${ }^{(14)}$ in the mid 1950s. General analytical methods for wing-jet interaction of various basic configurations were then presented by $\operatorname{Lan}^{(18)}$ and Shollenberger ${ }^{(19)}$ in the early 1970s.

For this work, the use of the term "blowing" will refer to either blowing from the trailing edge or blowing across the upper surface of a wing or airfoil. There are certainly other types of blowing, but the fundamental interactions are similar. At this point it is also critical to establish our correlating parameter, the "blowing coefficient" or "jet momentum coefficient":

$$
C_{\mu}=C_{j}=\frac{\dot{m} V_{j e t}}{q_{\infty} c}
$$

where the numerator is the gross thrust of a propulsion system operating at or near design condition (jet static pressure equal to ambient pressure). This parameter is a measure of the strength of the jet.

A distinction should also be made between blowing for boundary layer control and for powered lift. Boundary layer control is the process of blowing to prevent boundary layer separation. It typically uses very small amounts of air. As we begin to use more air, the jet leaves the trailing edge with some small amount of momentum. At this point, call it $C_{\mu, \text { crit }}$, the boundary layer control has become powered lift. The increase in airfoil circulation due to the jet is called "super-circulation". There are two ways in which blowing will alter the performance of an airfoil:

1. Through the direct reaction of jet momentum leaving the trailing edge.

2. Through the change in airfoil circulation induced by the jet, i.e. through the changes induced in the surface pressures around the airfoil. 
The first is obvious, while the second, as we will show, can be complicated. It involves many separate effects that are dependent on a wide array of flow features.

We begin with a discussion of trailing edge blowing. If this jet can be directed downward, it is called a "jet-flap" and has been the subject of a great deal of analysis, most notably that done by Spence ${ }^{(14)}$. It is helpful to re-derive the Kutta-Joukowski theorem (Eq. 2-1) as it applies to these cases. Consider the situation of a self-propelled, 2D wing in a uniform, steady flow. Ignore body forces and assume that the propulsion system operates at the design condition. A representative sketch of the body and control volume are given in Figure 2-2:

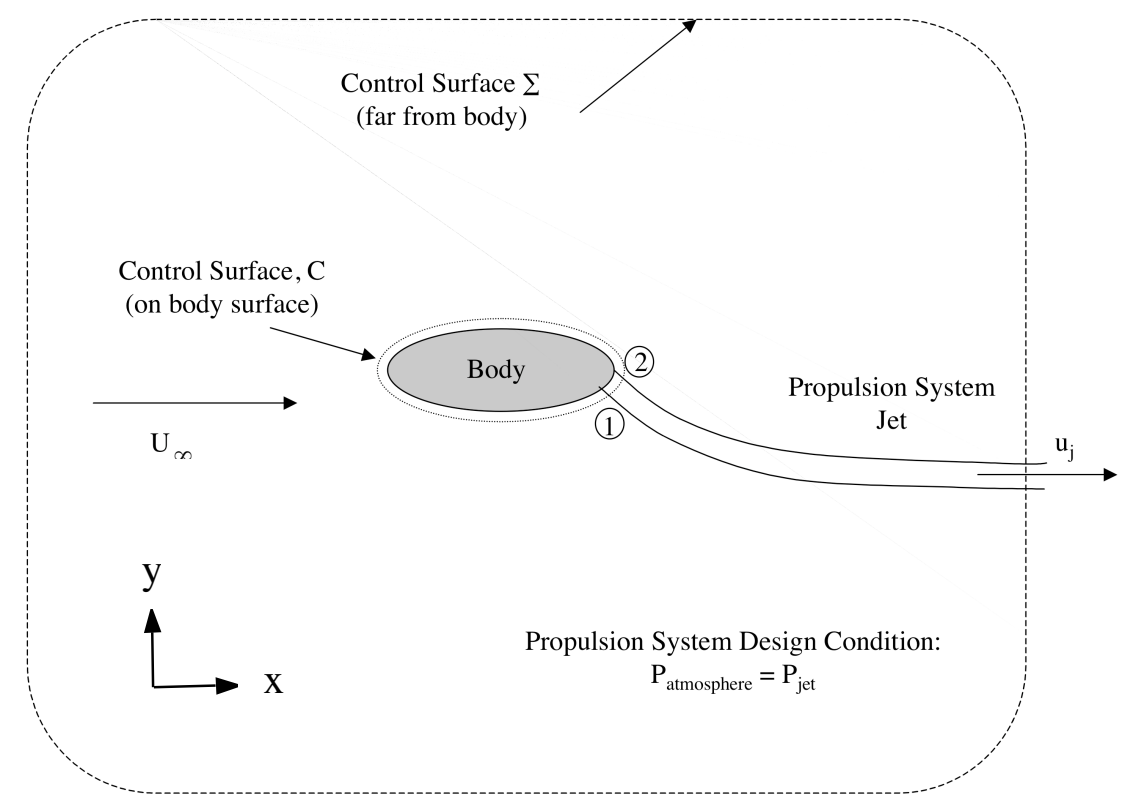

Figure 2-2: Sketch of blown section and control surfaces

For self-propelled bodies, forces are generated by surface pressures and the jet reaction. Following the general derivation from Karamcheti ${ }^{(20)}$, the force on this body is:

$$
\mathbf{F}=-\oint p \mathbf{n} d c-\dot{m}_{p}\left(u_{j}-U_{\infty}\right) \mathbf{i}
$$

where $p$ is the pressure, $d c$ is a differential length along a control surface, and $m_{p}$ is the mass flow rate per unit span. At this point, an expression can be substituted for the 
pressure (Karamcheti, eq 10.62) and the behavior of this new expression examined using the far-field control volume, $\Sigma$. Both of the control surfaces enclose the same body, making them irreducible, reconcilable circuits. In this case, it is known that the circulation around all such circuits has the same value. Thus, knowing the integral's value over the far-field control surface, the integral's value is known over any surface enclosing the body, including one which is right on the body surface. The force expression is found to be (see eq. 11.92 in Karamcheti):

$$
\mathbf{F}=-\rho_{\infty} U_{\infty} \times \oint(\mathbf{n} \times \mathbf{q}) d c-\dot{m}_{p}\left(u_{j}-U_{\infty}\right) \mathbf{i}
$$

where $\mathbf{q}$ is the "disturbance velocity", i.e. the difference between the total fluid velocity at a point and the freestream value.

Since $\mathbf{V}$ is the vector sum of $\mathbf{U}$ and $\mathbf{q}$, and the condition of no flow through the body must be satisfied over the majority of the surface, the following is assumed:

$$
\oint \mathbf{n} \times \mathbf{U}_{\infty} d c=0
$$

and,

$$
\oint \mathbf{n} \times \mathbf{q} d c=\oint \mathbf{n} \times \mathbf{V} d c
$$

The contour integral of the cross product of the normal and velocity vectors is easy to recognize as being the circulation around the airfoil. However, in this case, there are two discrete portions on our contour. The majority of the surface has the typical no-flow boundary condition, while a (typically) small portion has an outflow boundary condition and thus has a different velocity. Referring to Figure 2-2, break the contour integral into 2 pieces:

$$
\mathbf{F}=\left[\rho_{\infty} U_{\infty} \times \oint_{1 \rightarrow 2}(\mathbf{n} \times \mathbf{V}) d c\right]+\left[\rho_{\infty} U_{\infty} \times \oint_{2 \rightarrow 1}(\mathbf{n} \times \mathbf{V}) d c\right]-\dot{m}_{p}\left(u_{j}-U_{\infty}\right) \mathbf{i}
$$


The contour integral in the first term of equation (2-7) is approximately equal to the standard circulation value, $\Gamma$, although the presence of the jet will enforce a different trailing edge condition and thus a modified circulation, $\Gamma_{\text {mod }}$. The second contour integral can be evaluated since the velocity conditions at the jet surface are known. If the jet is undeflected, the normal and velocity vectors are parallel and this term becomes zero. If the jet is given a deflection angle, $\delta$, relative to the chord line this term becomes:

$$
\rho_{\infty} U_{\infty}\left(u_{j} t \sin \delta\right)
$$

where $t$ is the jet thickness, i.e. approximate contour length from 2 to 1 . This term represents the contribution of the reaction force to lift. It is important to note that for upper surface blown sections, the contour integral from 2 to 1 may also include significant portions where the jet runs parallel with the surface. Thus, the velocity vector is tangent to the surface and can alter the force in that fashion as well.

The total force on the body can then be written as:

$$
\mathbf{F}=\rho_{\infty} U_{\infty} \Gamma_{\bmod } \mathbf{k}+\rho_{\infty} U_{\infty}\left(u_{j} t \sin \delta\right) \mathbf{k}-\dot{m}_{p}\left(u_{j}-U_{\infty}\right) \mathbf{i}
$$

This is the analogy to the Kutta-Joukowski theorem for a blown airfoil. It simply states that the jet will modify the basic circulation (super-circulation) and any surface on which it acts, and it will produce a net thrust.

A derivation done by Siestrunck ${ }^{(21)}$ comes to an analogous expression:

$$
\mathbf{F}=\rho U \Gamma \mathbf{k}-\left(p_{j}+\rho_{j} V_{j}^{2}\right) \delta \mathbf{i}
$$

where $\Gamma$ is the total circulation of the wing-jet system, and the second term describes the reaction lift force. (Note $\delta$ in eqn. 2-10 denotes jet width)

While the induced flow is similar to that of a simple mechanical flap, it is important to understand where and why changes occur. In a subsonic flow, changing the trailing 
edge conditions will alter the entire flowfield. Figure 2-3 shows airfoil surface pressures with and without trailing edge blowing.

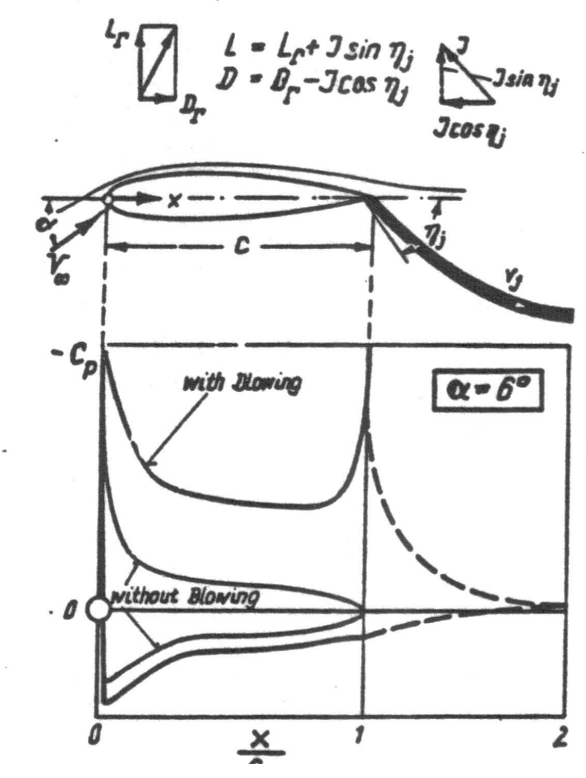

Figure 2-3: Pressure distribution on a jet-flapped airfoil from Ref 16

The presence of the jet has significantly altered the surface pressures. The curvature of the flow due to the presence of the jet induces a similar type loading as a mechanical flap. This explains a great deal of the change between the pressure distributions, but there is more to be seen. Consider the situation shown in Figure 2-4.

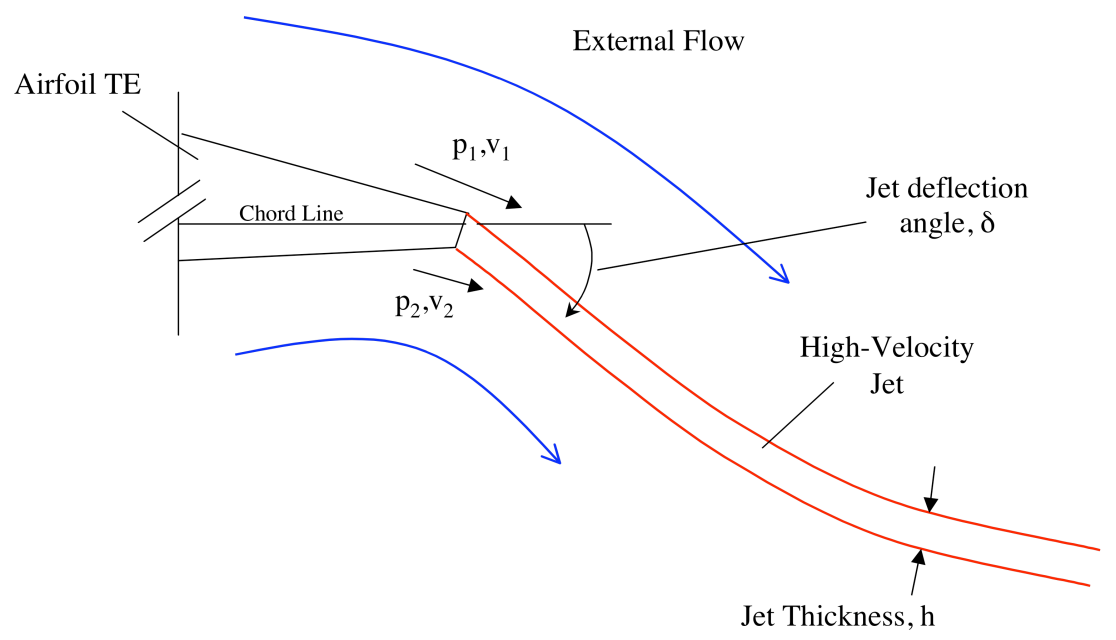

Figure 2-4: Sketch of the trailing edge of a jet-flap airfoil 
The curvature of the jet sheet allows it to support a pressure difference across it, and thus in a typical situation $p_{2}>p_{1}$ and by Bernoulli's equation, $v_{1}>v_{2}$. Having the jet sheet at the trailing edge effectively relaxes the classical Kutta condition, allowing the upper surface and lower surface to come to different velocities at the trailing edge. Siestrunck says, "The thin jet sheet plays the part of a regulator of the circulation as would the sharp trailing edge of a solid flap". Thus, the adverse pressure gradient is relieved or even changed to a favorable one on the aft portion of the upper surface. This is another increase in circulation. This effect could be seen even if the jet-flap angle were zero, as will be demonstrated in later chapters.

It is worthwhile to contrast this process with that seen in "circulation-control" airfoils, on which a significant amount of work has been done by Englar ${ }^{(22)}$. These airfoils use trailing edge blowing over a thick, rounded trailing edge. Very large increases in circulation are seen even though the jet doesn't play the same role as with jet-flaps or upper surface blowing. In these cases, the circulation increase comes directly from controlling the location of the airfoil's stagnation points. In the present case, circulation is gained by providing a different trailing edge condition. For this reason, higher $C_{\mu}$ values are required to achieve performance than needed for a circulation control wing.

The pressure difference across the jet plays another important role. The force it creates on the jet acts to turn the jet back into the direction of the freestream. Helmbold ${ }^{(23)}$ says that the turning force must be in equilibrium with the excess of centrifugal acceleration in the jet. Theoretically, at infinity downstream of the airfoil, the jet becomes parallel to the freestream regardless of its initial deflection. Since drag is zero in inviscid 
flow, the leading edge thrust and the drag component of the turning force are equal and opposite. Thus, in inviscid flow, it is assumed that we have full thrust recovery.

\section{3 - Effects of Surface Blowing}

The situation becomes more complex viscous effects are included. The following figure shows a drawing of a typical USB configuration including some of the viscous effects:

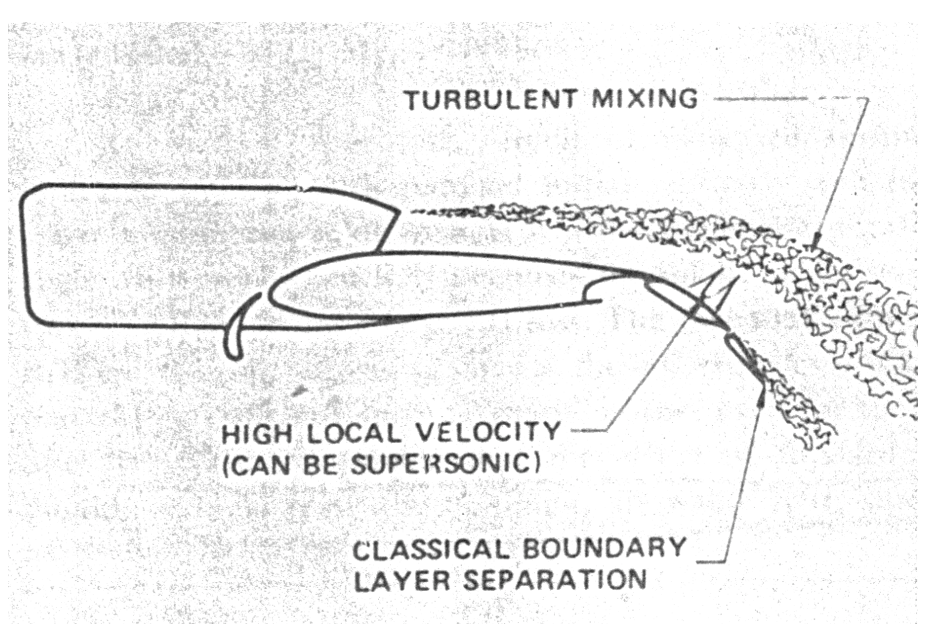

Figure 2-5: USB configuration from Davenport ${ }^{(24)}$, reprinted with AIAA permission Clearly shown are the viscous mixing layer and the classical boundary layer separation. What can't be illustrated is the effect of direct fluid entrainment, by which the jet induces pressure gradients forward of the nozzle that accelerate, or entrain, the fluid. It should also be noted that although it is required that the static pressure is continuous at the jet flow/external flow boundary, the jet is operating at a higher total pressure, $p_{o}$. Thus, some portion of the airfoil surface is effectively seeing an entirely different flow.

To better understand the effects of surface blowing, begin with a brief discussion of shear layers, turbulent wall jets, and the Coanda effect. Shear layers are discussed by Schetz ${ }^{(25)}$ and refer to viscous regions between moving fluids. In the case of a single fluid 
with regions moving at different velocities, a layer of fluid is present between the flows in which the velocity profile transitions smoothly. Similar to a boundary layer, this region grows as momentum is diffused.

Turbulent wall jets have been studied for quite some time due to their many practical applications. Of particular interest here are turbulent wall jets flowing over convex surfaces. Launder and Rodi ${ }^{(26)}$ provide a very good description of the phenomena involved. Directly related to this subject is the Coanda effect, which is the remarkable ability of a wall jet to remain attached to the adjacent curved surface even when the surface subtends a large angle. The turbulent wall jet basically consists of an inner region where the velocity behaves like a turbulent boundary layer, and a much larger outer region that is similar to a shear layer.

According to Launder and Rodi, the Coanda effect is caused by momentum transfer between the inner and outer regions. Essentially, the convex streamlines intensify turbulent mixing in the outer region and reduce it in the inner region. This increased outer layer mixing is a cause for the increased rate of jet growth over a curved surface as compared to a planar one. The process of "selective amplification" of turbulent mixing increases the importance of turbulent diffusion in the jet.

Consider now the case where a jet engine exhausts tangentially at some point on the upper surface, instead of at the trailing edge. (The issue of aft flaps will be discussed later) As the relatively thick jet proceeds over the curved airfoil, it behaves similarly to a wall jet over a curved surface, but we have the additional complication of a non-uniform external flow. The wall jet will induce lower pressures over the wetted surface. It will also experience viscous losses due to increased mixing as it turns across the surface and 
due to usual boundary layer effects. Thus, there is a reduction in the momentum flux (thrust) exiting the trailing edge.

In addition, there are entrainment effects. In properly designed cases these effects can become quite large. This is the idea behind thrust augmenters ${ }^{(27)}$, where entrainment effects effectively replace an actuator disk as the source of generating a pressure differential. In the present case, the effects are secondary and have not been studied significantly. The viscous interaction between the jet and the external flow can relieve adverse pressure gradients upstream of the nozzle exit. This can provide a significant increase in airfoil circulation.

Thus, basic viscous interactions can greatly complicate the problem. Although the momentum flux leaving the trailing edge is less than would exist if the jet were located at the trailing edge, a significant increase in circulation can be gained by surface blowing. However, one must also take into account the losses that these viscous processes introduce. An outline for thrust/drag bookkeeping is given in the next chapter.

The effects of trailing edge flaps arise naturally from the ideas just discussed. Figure 2-6 is a sectional view of the YC-14's propulsion system/USB flap.

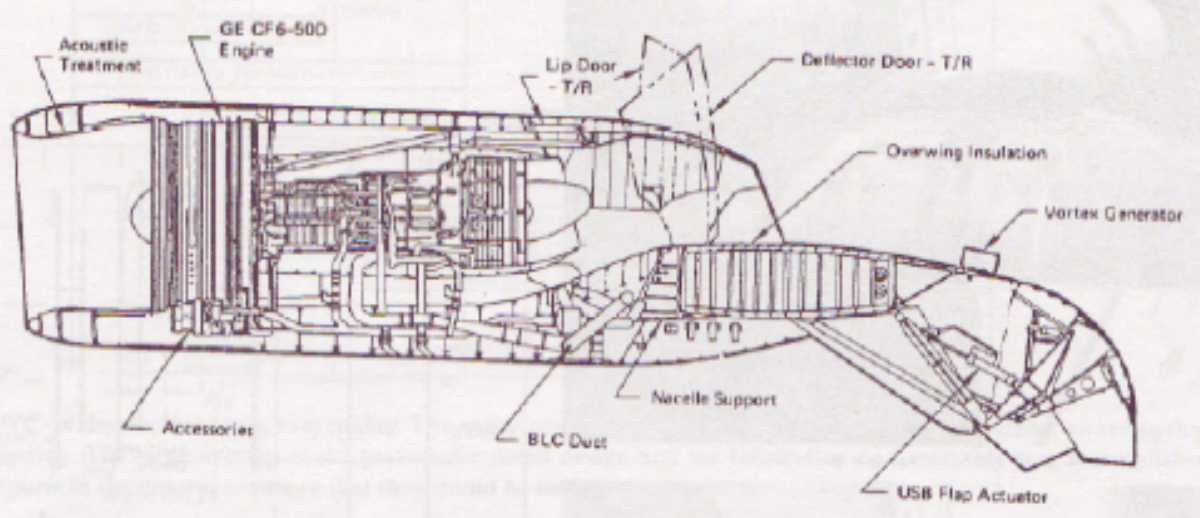

Figure 2-6: YC-14 USB nacelle/nozzle/flap system from Wimpress ${ }^{(5)}$, reprinted with AIAA permission 
Notice that the elements of the flap system move and orient themselves in such a way as to provide a continuous, curved surface for the jet. The primary parameters are the final trailing edge angle and the curvature of the flap surface. Contrast this design to the multielement systems discussed in Reference 15 where element gap and overlap are the primary parameters. The large curvatures of deflected flaps follow the same rules as described above, however the viscous processes become more pronounced with flap deflection.

As the curvature becomes large, the jet is turned through a larger angle. The viscous shear/mixing layer grows large and can eventually breakdown the Coanda effect and cause the jet to separate from the surface. The large curvatures also lead to lower surface pressures on the flap. This provides the characteristic increases in lift force, but also provides a drag force as the surface normal vector rotates away from vertical. Some of this drag force is never realized as the leading edge suction effect increases with circulation, but it is clear that USB flaps can produce large amounts of drag.

\section{4 - Breakdown of Forces}

Consider the sketch shown in Figure 2-7 of an idealized USB configuration. No account is made for a propulsion system inlet. 


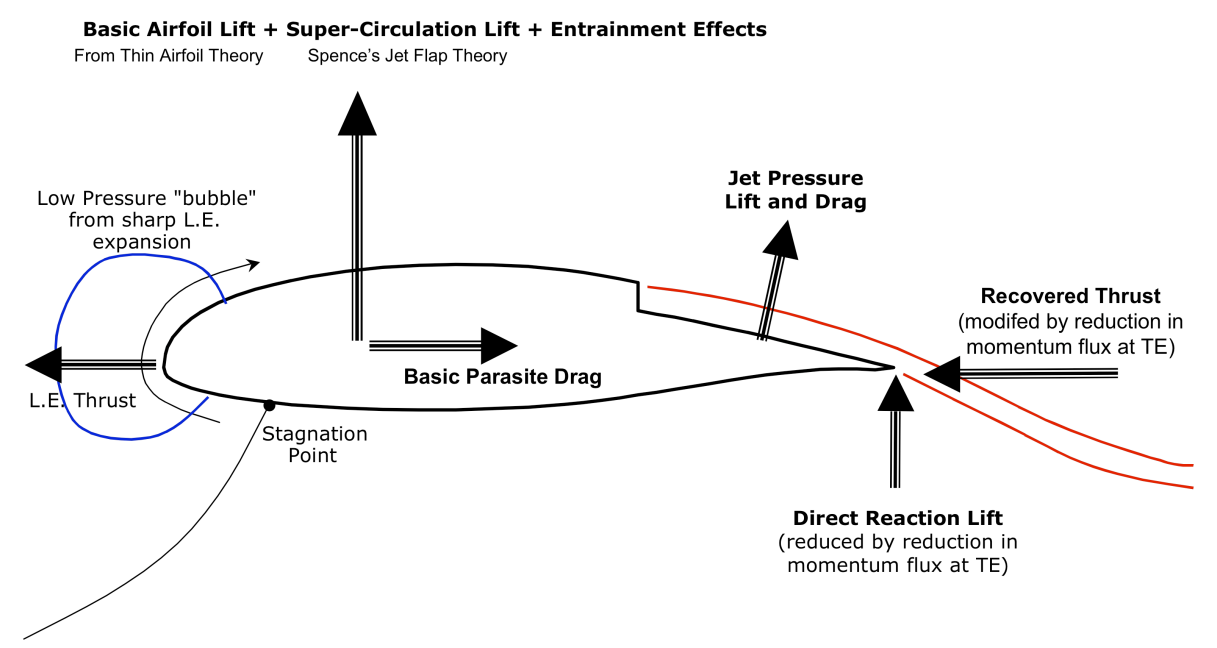

Figure 2-7: Sketch of forces for an idealized USB configuration

The main lift force is taken to act through the quarter chord. It is supplemented by a distributed force over the variable-length aft surface, and the direct reaction lift. Thus, the naming system used for lift is:

$$
\begin{array}{ll}
C_{l, \text { basic }}= & \text { basic, un-blown lift coefficient } \\
C_{l, j e t f l a p}= & \begin{array}{l}
\text { additional lift due to presence of a pressure discontinuity at the } \\
\text { trailing edge. }
\end{array} \\
C_{l, \text { pressure }}= & \begin{array}{l}
\text { lift from distributed pressure force on the aft surface and/or } \\
\text { USB flap system }
\end{array} \\
C_{l, \text { direct }}= & \quad \begin{array}{l}
\text { direct lift from jet momentum reaction } \\
\eta_{\text {ent }}=
\end{array} \quad \text { factor on the total lift due to entrainment effects }
\end{array}
$$

Naturally, in powered lift configurations, the drag force is very closely related to the thrust. Chapter 4 will discuss this in more detail. For now, the breakdown of horizontal forces experienced by the airfoil is:

$$
\begin{aligned}
& C_{d, \text { basic }}=\quad \begin{array}{l}
\text { drag from the basic airfoil section with no blowing, including } \\
\text { pressure drag and skin friction drag }
\end{array} \\
& C_{d, \text { pressure }}=\quad \begin{array}{l}
\text { drag from the distributed pressure force on the aft surface and/or } \\
\text { USB flap system }
\end{array} \\
& C_{T, \text { recovered }}=\quad \text { the thrust recovered infinitely downstream in a real case }
\end{aligned}
$$




$$
C_{T, L E}=\text { the suction force at the leading edge }
$$

Once the lift and drag forces are understood, the pitching moment for the USB section becomes much clearer. It has been well documented that upper surface blowing causes large, nose-down pitching moments. Looking at Figure 2-7, it is easy to see why. Any airfoil modification that produces large chord loadings near the trailing edge will have large nose-down pitching moments. An example of this is adding "aft-camber" to an airfoil. Upper surface blowing does significantly modify the aft chord loading, and it experiences the reaction moment from the jet leaving the trailing edge. The breakdown for the pitching moment is very similar to that of the lift:

$$
\begin{aligned}
& C_{m, b a s i c}=\quad \begin{array}{l}
\text { basic moment coefficient } \\
C_{m, \text { jet flap }}=
\end{array} \begin{array}{l}
\text { additional moment due to presence of pressure discontinuity at the } \\
\text { trailing edge. }
\end{array} \\
& C_{m, \text { pressure }}=\quad \begin{array}{l}
\text { moment from distributed pressure force on the aft surface and/or } \\
\text { USB flap system }
\end{array} \\
& C_{m, \text { direct }}=\quad \text { direct moment from jet momentum reaction at the TE }
\end{aligned}
$$

\section{5 - Adverse Effects}

The purpose of this section is to describe the processes that occur in a USB design that can decrease the performance. A large amount of experimental work has been done to find factors that contribute to these processes and what can be done to minimize them. The primary concern relates to the jet's ability to turn efficiently over the flap. Also of concern is the behavior of the leading edge when the airfoil is at high values of supercirculation. As described by Phelps ${ }^{(28)}$, the low speed performance appears to be mainly 
dependent upon the jet turning angle and turning efficiency and on adequate leading edge treatment to prevent premature flow separation.

When referring to a thick jet's ability to follow a curved surface, two important parameters must be defined clearly. First, define the turning angle, $\delta$, as the angle through which the exhaust jet actually turns. Second, define the turning efficiency, $\eta_{\text {turn }}$, as the measure of how much of the static thrust is achieved at a given turning angle. In other words, given a certain jet deflection, how much of the static axial thrust is successfully turned through the angle?

The turning angle is essentially a reflection of the curvature achieved in the mixing/shear layer. A great deal of mixing means that the jet boundary is growing thicker and the curvature is reduced. This means that the jet will not have turned through the desired trailing edge angle. A study from the $\mathrm{NADC}^{(29)}$ contains a great deal of collected and summarized turning data. The turning angle is primarily affected by:

1. The exhaust flow angle relative to the surface (this is referred to as the "kickdown" angle). A high nozzle kick-down angle will force the exhaust jet to be thinner.

2. The ratio of jet thickness to turning radius. A thin jet will turn much more efficiently than a thick jet, making low values of this ratio desirable.

3. Nozzle design. This is a broad category that includes both the internal design and external design

4. Boundary layer condition over the turning surface

Clearly, the design of the nozzle to achieve a thinner jet with minimal losses is crucial for upper surface blowing.

The turning efficiency is closely tied to the turning angle. Essentially, the jet begins losing total pressure due to the mixing and turning process. Portions of the jet's energy are being absorbed by viscous processes in the boundary layer and in the mixing/shear layer. As shown in the experimental data of Ref 28, turning efficiencies can approach 100 
percent for small flap deflections. However, for large flap deflections, the turning efficiency can drop to about 80 percent. This is still a favorable efficiency compared to some other powered lift designs such as externally blown flaps ${ }^{(30)}$.

The other concern noted above is that of leading edge flow separation. Referring again to Figure 2-7, note that the stagnation point has been shifted aft on the lower surface due to the high values of circulation. This induces the rapid leading edge expansion shown. However, if this expansion becomes too severe, the flow may separate at the leading edge. The correction used in all practical designs has been to incorporate leading edge flaps or slats to soften this expansion and permit higher values of circulation. This does slightly diminish the lift coefficient at a given configuration, but permits a higher maximum lift coefficient. Another suggested solution by Englar ${ }^{(22)}$ is to incorporate leading edge tangential blowing (boundary layer control). This would prevent separation and actually augment the pressure distribution.

\section{6 - Three-Dimensional Behavior}

While the analytical methods developed in this work are primarily for two dimensions, it is useful to discuss the three-dimensional behavior of USB configurations. As expected, the 3-D case cannot perform as well as the 2-D case. This is partially due to the fact that nearly all USB designs (and certainly all those built to this point) can only blow over limited portions of the wingspan. Nozzles designed to spread the exhaust flow laterally are desirable, but this spreading can incur losses if not done properly. For this discussion, only partial-span blowing designs are considered. 
As described by Phelps ${ }^{(28)}$ and demonstrated in other configuration data, upper surface blowing exhibits a "localized" flow behavior. This means that there is very little lateral spreading of the jet. Contrast this with the behavior of externally blown flaps where the jet impinges on the lower surface of the flap and spreads spanwise. In a USB configuration, the jet influence is confined to a localized region by the formation of vortices on the edges of the jet sheet.

Perhaps the most significant decrease in performance comes from induced drag. As described by Mason in Reference 15, the induced drag is a function of the spanloading. The localized nature of USB leads to relatively sharp changes in the spanloading, which in turn can lead to higher values for the induced drag. While this is offset by the enormous gains in lift performance, it can still be an issue.

The full three-dimensional problem has been studied using modified panel methods $\left(\right.$ Narain $\left.^{(31)}\right)$, zonal methods (Roberts $\left.{ }^{(12)}\right)$, and wing-wake vortex lattice methods (Mendenhall $\left.{ }^{(13)}\right)$. However, these methods can be costly to set up for a given configuration. For example, the panel methods must be determined for the configuration including special paneling schemes for the nacelle and flap surface. In addition, an iterative scheme must be used to determine the jet position given the specific propulsion conditions. The zonal method, while it has the potential to be a very accurate method, is very costly to set up due to the fact that a very complex grid must be developed for even a basic problem. The vortex lattice method was more successful, but still was hard to use and often significantly over-predicted the wing lift performance. 


\section{Chapter 3: Thrust/Drag Bookkeeping for USB Designs}

The closely coupled interactions between the aerodynamics and propulsion in powered-lift configurations can be a major problem area for a design. The idea of integrating the propulsion system into the wing has been around since the advent of jet propulsion. Creating efficient integrated designs is the essence of multidisciplinary design optimization (MDO). The design of efficient wing/nacelle combinations is the first step toward fully integrated propulsion/aerodynamic design. Powered-lift aircraft will never achieve their full potential without an integrated design approach.

There are two main purposes of this chapter. The first is to present the different areas where losses should be accounted for. This will draw on the discussion of Chapter 2 regarding the flow physics involved. A possible "bookkeeping" system for powered-lift is also suggested. The second purpose is an attempt to establish simple empirical methods for estimating these losses. While no one way is absolutely correct, we can reduce confusion by addressing these areas and being consistent. 


\section{1 - Losses and Bookkeeping}

Following Rooney ${ }^{(32)}$, for a steady flight state a balance exists between the installed propulsive force and the drag:

$$
F_{I P F}=D
$$

This installed propulsive force includes throttle dependent forces. In the case of USB, this is a little more vague. It is desirable to eliminate as much throttle dependence as possible from the drag polars, however, unlike conventional configurations the lift is primarily throttle dependent. The following losses dominate USB aero/propulsion integration:

1. Nozzle losses - These losses come from the need to get a high aspect ratio "thin" jet from an annular engine flow.

2. Scrubbing losses - "Scrubbing" refers to the action of the turbulent jet on an adjacent surface. This is typically the upper wing/flap surface but can include the fuselage.

3. Mixing/flow turning losses - These losses are possibly the hardest to study analytically. This is because they are essentially losses due to turbulent momentum transfer with both the external free-stream flow and the boundary layer.

4. Thrust Recovery - Thrust recovery refers to the ability of a deflected jet to return parallel to the free-stream velocity.

A basic look at these processes shows that they are all throttle dependent. However, some of them may contribute to the lift and pitching moment. The primary example is the process of scrubbing, which gives an additional form drag, while providing significant contributions to lift and pitching moment. This problem was identified by Bowers ${ }^{(33)}$ for the Quiet Short-haul Research Aircraft (QSRA). Based on this argument, it is suggested that any mechanism that contributes significantly to both lift and drag will be included in the polar even though it may be strongly throttle dependent. 
Thus, the installed propulsive force would consist of:

$$
F_{I P F}=T_{\text {ref }}+\Delta T_{\text {nozzle }}+\Delta T_{\text {turning }}+\Delta T_{\text {downstream }}^{\text {recovery }}
$$

where $T_{r e f}$ is the thrust including any cruise nozzle penalties, $\Delta T_{\text {nozzle }}$ represents any losses due to changing the nozzle shape (such as increasing aspect ratio for high-lift purposes), $\Delta T_{\text {turning }}$ represents losses associated with flow mixing, and the last term represents any losses due to incomplete thrust recovery. These are throttle-dependent, but they do not contribute to lift/pitching moment.

The drag force is:

$$
D=D_{\text {base }}+\Delta D_{\text {scrubbing }}+D_{\text {induced }}
$$

where $D_{\text {base }}$ consists of the normal sources of aerodynamic drag, $\Delta D_{\text {scrubbing }}$ is the additional friction/form drag due to scrubbing, and $D_{\text {induced }}$ represents the induced drag from circulation shed into the wake. While the scrubbing term is clearly throttledependent, it is also a source of lift/pitching moment. Note that "ram" drag is not accounted for in this analysis.

This form of bookkeeping is somewhat different than some seen in other papers, where the drag polars reflect the whole "Thrust+Drag" term. There are advantages to viewing the polars this way. First, for a given throttle setting, it shows where a limit exists for level flight (when thrust plus drag becomes positive). Second, since one is basically looking at "excess thrust" or "excess drag", the flight path angle is easy to determine. Thus, for a given climb angle/approach angle and a given power setting, the lift coefficient necessary is found from the drag polar plot. Figure 3-1 shows drag polars taken from Sleeman $\&$ Holwheg ${ }^{(6)}$ for a USB aircraft with $35^{\circ}$ flap deflection. 


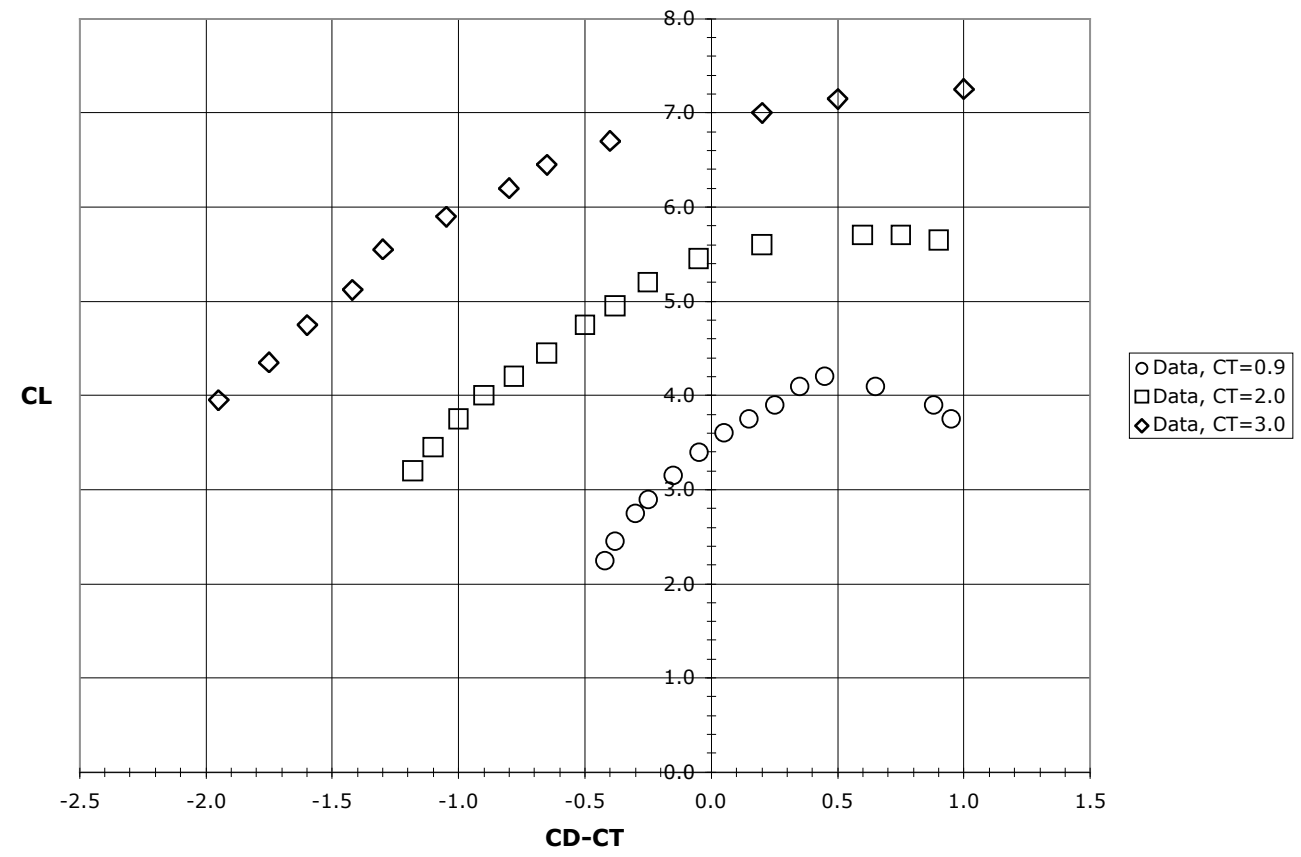

Figure 3-1: Drag Polars for a four-engine USB configuration at 35 deg flaps

However, the plots can be misleading. They give no impression of how the drag itself is changing with increasing power setting. In a similar fashion, the thrust losses are implicit in the "thrust+drag" term. The aerodynamic drag can be obtained from these plots only when the thrust and associated thrust losses are known so they can be removed. In addition, most design codes accept input of the aerodynamic drag only, so the thrustplus-drag values would have to be separated manually before they would be usable. For these reasons, we will keep the two quantities separated as much as possible using the procedures presented above. When presenting comparisons, the familiar thrust-plus-drag format will be used, but only after the individual calculated quantities are combined. Thus, the individual contributions are made clear.

\section{2 - Estimating Losses}

This section deals with finding ways to estimate the losses discussed above for a given configuration and propulsion state. These estimates draw heavily on published 
experimental data, from which empirical relations can be developed. If data for a specific configuration is available, it will supercede these relations.

First, look at losses developed by the nozzle. Depending on the nozzle design, the magnitude of these losses can vary significantly. Jet shape is crucial to USB performance, but nozzle exit area must correspond to the engine flow condition. Previous designs have been forced to incorporate nozzle geometry-changing schemes to satisfy different conditions. These losses are counted against the propulsion system. In the case of the YC14 , nozzle effects were treated as losses to allow the "reference" thrust of the engine to be that of the engine by itself. Some aircraft design software has internal routines for estimating nozzle losses, but these losses will not be estimated as a part of this work.

The next category is scrubbing losses. These include the skin friction and induced surface pressures. As mentioned before, since these tie most closely to the aerodynamic performance, these effects will be accounted for by aerodynamics and will not affect propulsion. The skin friction will be discussed more thoroughly in Chapter 4, as will a method for predicting the induced surface pressures. There can be significant contributions to the lift, drag, and pitching moment from these local pressures.

We can now discuss the turning losses, which come from the reduction of curvature the flow experiences due to mixing. Completely understanding the process causing these losses could require extensive numerical analysis. Fortunately, since these losses are the main factor in how well a jet turns over a Coanda flap, there is a lot of test data upon which to base an empirical approximation ${ }^{(29),(34),(35)}$. The turning angle was defined previously as the actual angle through which the exhaust jet is turned compared to the 
flap angle. Figure 3-2 demonstrates the nozzle kick-down angle, $\theta_{k d}$, which is a driving factor in turning angle efficiency.

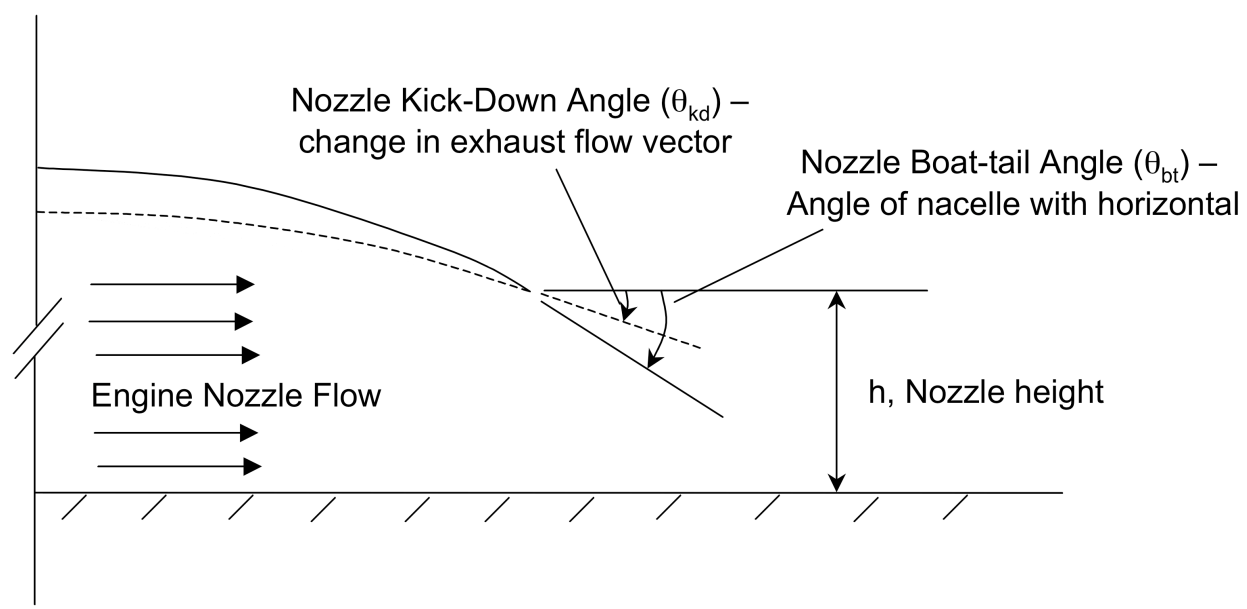

Figure 3-2: Sketch of nozzle exhaust flow angles

Based on results presented in Reference 29, the achievable flow turning angle is a strong function of the kick-down angle and the ratio of jet thickness to flap curvature, $h / R$. For typical height-to-radius ratios of 0.3 or less, the flow turning angle can be approximated using:

$$
\delta_{j}=\delta_{f}\left[1-\exp \left(\left(-10+29.3(h / R)-\left(0.567(h / R)\left(\theta_{k d}(\mathrm{deg})\right)\right]\right.\right.\right.
$$

Clearly, better jet turning comes from having a thinner jet. Figure 3-3 shows experimental data for $h / R=0.3$ along with the empirical relation of equation 3-4. It should be noted that cases having larger $h / R$ ratios would require more empirical development, and equation 3-4 should not be used. 


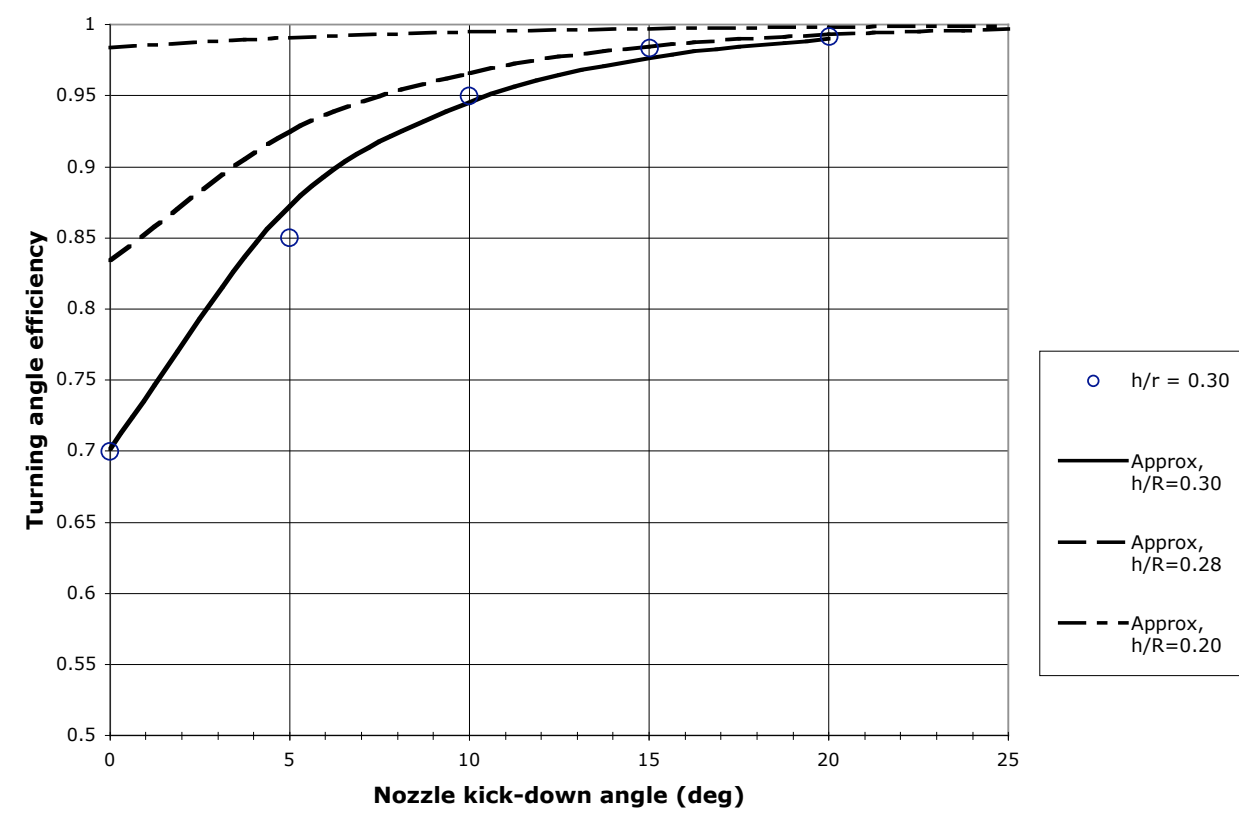

Figure 3-3: Comparison of experimental flow turning angle with empirical relation Similarly for turning efficiency, define:

$$
\eta_{\text {turn }}=\frac{T_{t e}}{T_{r e f}}=f\left(\delta_{j}\right)
$$

where $T_{t e}$ is the thrust magnitude at the trailing edge of the flap and $T_{r e f}$ is the thrust of the engine with cruise nozzle losses included. All experimental data show similar results for efficiency between 80 to $100 \%$ depending on deflection angle. This is quite high compared to some other powered-lift methods. Figure 3-4 demonstrates the typical range for USB turning efficiency data presented in a format adopted by most researchers in powered-lift. 


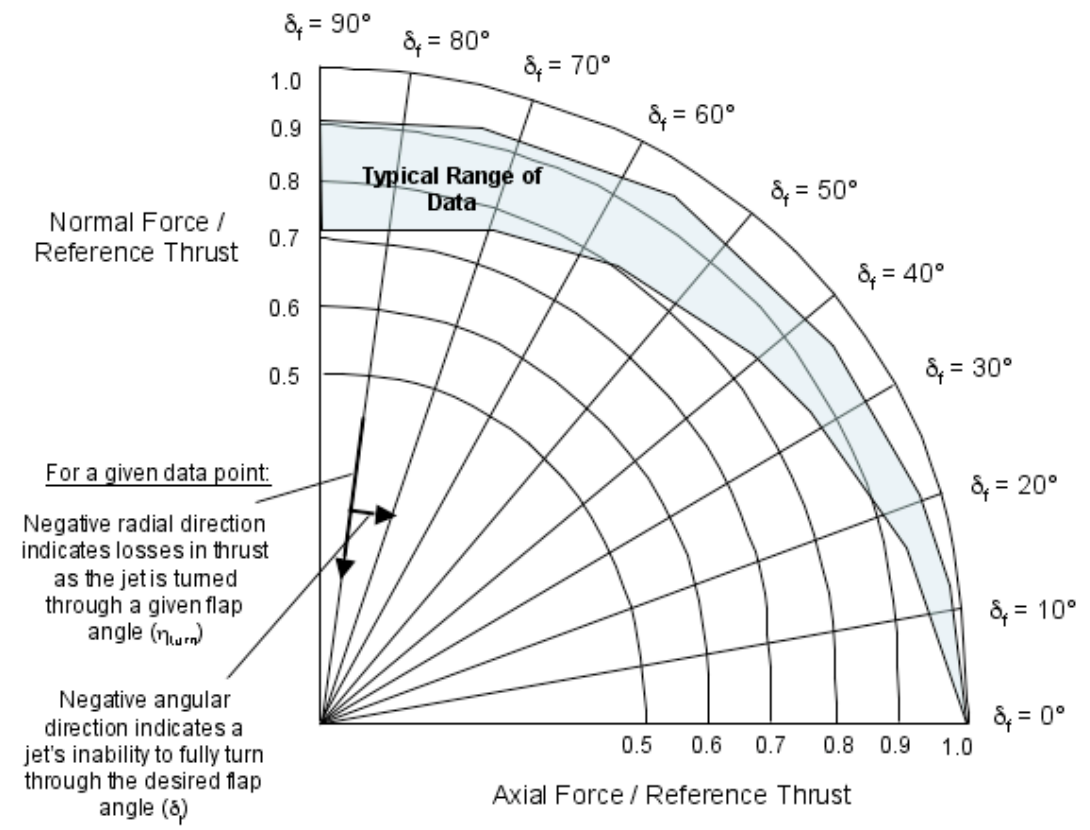

Figure 3-4: Typical turning efficiency range for USB flap systems

Based on results presented in reference 29 , a good linear empirical fit is:

$$
\eta_{\text {turn }}=1-0.1385 \delta_{j}(\mathrm{rad})
$$

It is also worth noting the effect of vortex generators on jet angle and turning efficiency.

These devices have been used on the YC-14 and ASKA, and their deployment produces a $10 \%$ increase in jet turning angle at a cost of 3-5\% of the turning efficiency. These losses should be counted against the propulsion system, since they make the most sense in that context.

Lastly, consider thrust recovery. In essence, thrust recovery means that the engine can be used to give lift without affecting thrust. Unfortunately, there will always be adverse effects on the resultant thrust due to the deflection. Flight test data from Boeing lists thrust recovery values ranging from 75 to $100 \%$ with varying USB flap deflections. This would also be counted against the propulsion system. A simple recovery factor can be 
applied to the thrust at the trailing edge. For flap deflections less than $\sim 40$ degrees, it will be assumed that there is a full thrust recovery. For deflections greater than 40 degrees, a recovery factor of 0.95 should be sufficient to approximate this effect. In most cases, assuming complete thrust recovery would alter results very little. 


\section{Chapter 4: Development of a Two-Dimensional Analytical Method}

The development of a methodology for USB calculations in two dimensions is the heart of the current work. The need for these types of calculations was described in Chapter 1. It was also shown that to be effective in the early design stages of an aircraft, any methodology should be fast, robust, and cost effective. In this chapter, we will discuss the basis for the method, explain it's constituent pieces, and introduce correlation factors. Chapter 5 will then present results of the method with published experimental data.

\section{1 - Basis for method / Overview}

Since the goal is to provide performance calculations early in the design process, the use of Computational Fluid Dynamics was effectively ruled out due to the amount of time needed to obtain CFD solutions. This is especially true when a configuration may be changing very quickly early in the design process. Thus, more basic inviscid methods were used. Viscous effects can then be approximated through correlation factors or empirical relations. For preliminary and conceptual design this is the general standard. 
Based on a survey of the literature available for upper surface blowing, and poweredlift in general (see Appendix A for an annotated bibliography), it was clear that classic jet-flap theory was inadequate for upper surface blown configurations. However, studies have been published by Hough ${ }^{(36)}$ showing that the "jet-flap analogy" can be very useful in predicting lift and pitching moment increments due to changes in geometry or flight state. As confirmed by Hough, Gainer ${ }^{(37)}$, and Lan ${ }^{(18)}$, it is expected that jet-flap theory under-predicts the total lift and pitching moment because it does not take into account the interaction between the wing and the jet, nor the general viscous nature of the jet flow.

Knowing this, if the surface pressures for those areas wetted by the jet can somehow be predicted, the total forces can be approximated much better. It was found here that Circular Streamline Theory (CST) provided a good approximation and was very easy to use. Its derivation will be shown later in this chapter. In addition, empirical relations were developed to approximate viscous losses and beneficial effects from entrainment. Figure 4-1 demonstrates the general method.

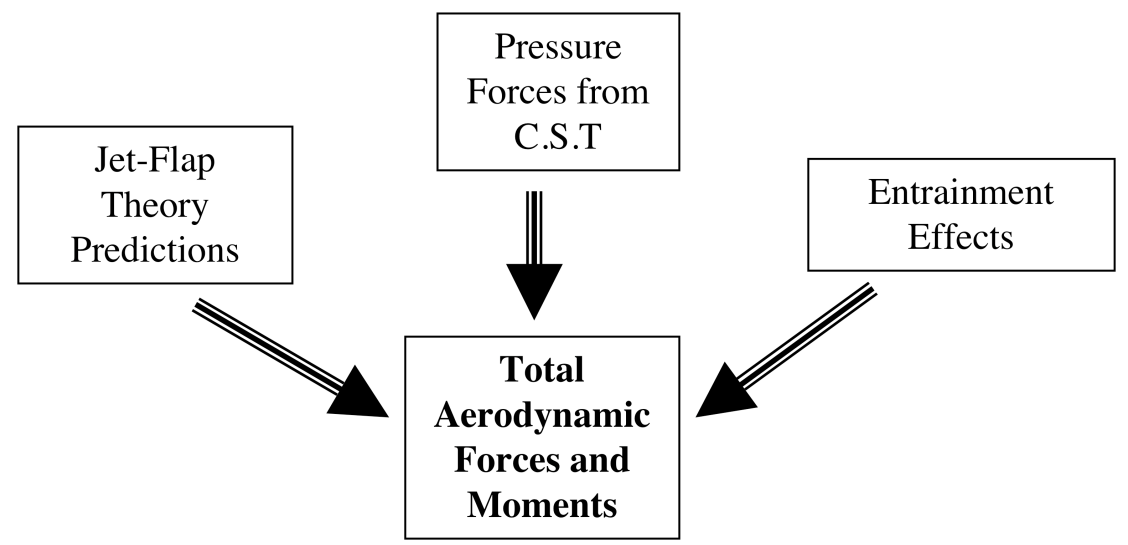

Figure 4-1: Schematic representation of current method

The following sections will deal with these individual components in formulating expressions for the lift, drag, and pitching moment of an upper surface blown airfoil. Finally, the complete 2-D equations will be presented. 


\section{2 - Spence's Jet-Flap Theory}

Classical Jet-Flap theory refers to the development by Spence ${ }^{(14),(38)}$ in the mid 1950s. His original work was for inviscid, incompressible flow past a thin, 2-D airfoil at moderate incidence with a thin jet exhausting from the trailing edge. It was later extended to include cases where the jet enters the flow tangentially along a deflected flap. The appeal of these methods comes from their success in analyzing jet-flap wings and from the simplicity of the method. For complete details of the analysis, the reader may refer to Spence's papers. A brief outline is given below.

Assume that both the main flow and jet flow are irrotational. Thus, similar to thinairfoil theory, a solution for a given flow can be obtained by constructing an equivalent problem involving fundamental singularities and imposing appropriate boundary conditions. The jet is modeled as being bounded by vortex sheets. There is no flow across the sheets, and the tangential velocity across the sheet is continuous. The jet's effect on the external flow is then the same as a vortex sheet whose strength distribution is governed by jet sheet's curvature and momentum flux.

Assuming that the jet sheet returns to the undisturbed freestream direction, Spence obtained an integro-differential equation for the problem. The solution was expressed using a Fourier series, together with a function that has the correct singular behavior at the trailing edge. Spence then formulated a 9-term interpolation to the series using a computer for values of the momentum flux coefficient, $C_{j}$, from 0.01 to 10 .

\subsection{1 - Lift Coefficient}

The lift coefficient is given as:

$$
C_{l}=2 \pi \alpha+2 \pi B_{o} \alpha+4 \pi A_{o} \tau
$$


where $\alpha$ is the angle of attack and $\tau$ is the jet deflection angle relative to the chord line. The coefficients $A_{o}$ and $B_{o}$ are the leading Fourier coefficients associated with jet deflection and angle of attack. A curve fit based on $C_{\mu}$ was given as:

$$
\begin{aligned}
& A_{o}=0.2817 C_{\mu}^{1 / 2}+0.0259 C_{\mu}+0.0124 C_{\mu}^{3 / 2} \\
& B_{o}=0.0917 C_{\mu}^{1 / 2}+0.0880 C_{\mu}+0.0041 C_{\mu}^{3 / 2}
\end{aligned}
$$

When a partial chord trailing edge flap is added, the equations must be modified slightly. Note that jet-flap theory does not account for interactions of the jet with the flap surface. The jet is essentially treated as coming from the deflected flap's trailing edge, and the flow along the surface provides the benefit of eliminating any flow separation. The sectional lift coefficient is given by:

$$
C_{l}=2 \pi \alpha+4 \pi B_{o} \alpha+2\left(\chi+\sin \chi+2 \pi D_{o}\right) \delta_{f}
$$

where $B_{o}$ is identical to that above. The variable, $\chi$, is defined as:

$$
\chi=2 \sin ^{-1}(\sqrt{E})
$$

where $E$ is the ratio of flap chord to airfoil chord. Implicit is the assumption that the jet angle is the same as the flap deflection angle, $\delta_{f}$.

Spence did not provide similar curve fits for $D_{o}$, since it is a complicated function of both $C_{\mu}$ and $E$. He simply presented the values graphically using families of curves for the different values of $E$. Based on these graphs, a multi-variable interpolated function was constructed for $D_{o}$ as part of the current work using non-linear regression analysis. The function obtained is:

$$
D_{o}=A_{o}+\frac{-1.931 E^{1 / 4}}{4 \pi}\left(C_{\mu}^{\left(-0.9621 E^{2}+0.5785 E+0.1639\right)}\right)
$$


Clearly, $A_{o}$ is the limit as $E$ goes to zero, i.e., as the flap becomes small the performance becomes more like a pure jet-flap. Figure 4-2 shows data points taken from Spence ${ }^{(38)}$ along with the interpolating function given in equation (4-6).

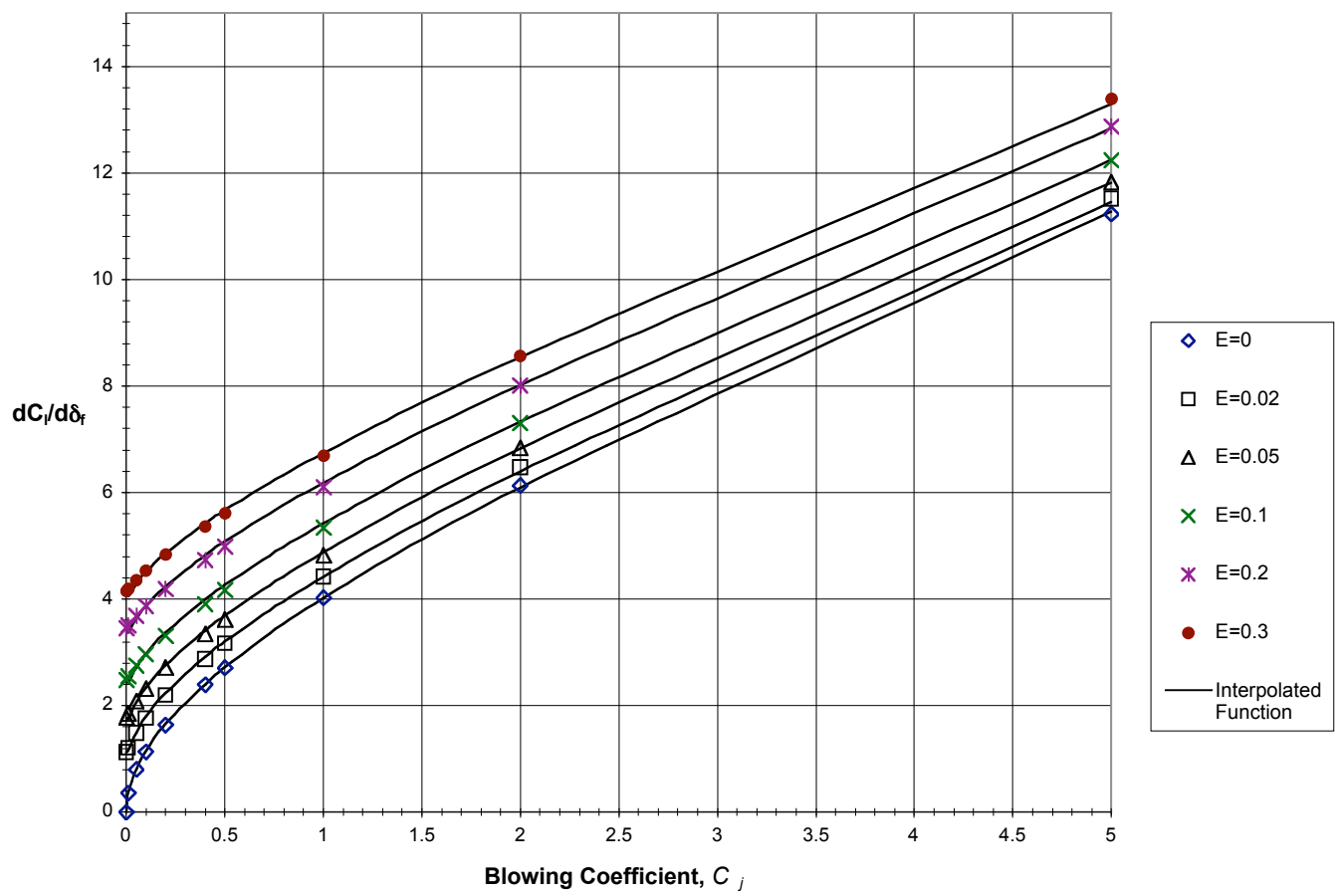

Figure 4-2: Agreement of $\mathrm{dC}_{\mathrm{l}} / \mathrm{d}_{\mathrm{f}}$ using derived approximate function (4-6)

The basic jet flap theory originally developed by Spence was extended by W. H. Davis $^{(39)}$ at Grumman Aerospace in 1979 to include camber effects. The study was limited to thin airfoils with parabolic camber. Note that this extension was originally done only for a pure jet flap. The sectional lift coefficient is given by:

$$
C_{l}=2 \pi \alpha+4 \pi B_{o} \alpha+4 \pi A_{o} \tau+4 \pi \gamma+4 \pi C_{o} \gamma
$$

where $\gamma$ is the airfoil camber as a percent of the chord, and $\tau$ is now referenced from the non-zero trailing edge angle. A curve fit for $C_{o}$ using similar powers of $C_{\mu}$ is :

$$
C_{o}=0.0600 C_{\mu}^{1 / 2}+0.4499 C_{\mu}-0.0922 C_{\mu}^{3 / 2}
$$


As clearly shown from comparing equations (4-1) and (4-7), the camber effect is purely additive via the last two terms of equation (4-7). This is not surprising, since this is a linear analysis thus superposition of solutions is valid. The first of the camber terms expresses the change in lift coefficient purely due to camber, the second (involving $\mathrm{C}_{\mathrm{o}}$ ) is dependent on the momentum coefficient. Combining the effects of a partial-chord flap with airfoil camber, the following expression is obtained for the lift coefficient:

$$
C_{l}=2 \pi \alpha+4 \pi B_{o} \alpha+2\left(\chi+\sin \chi+2 \pi D_{o}\right) \delta_{f}+4 \pi \gamma\left(1+C_{o}\right)
$$

\subsection{2 - Pitching Moment Coefficient}

In the case of a pure jet flap, the pitching moment is expected to be influenced by the blowing coefficient, $C_{\mu}$, through both the angle of attack, $\alpha$, and the jet deflection angle,

$\tau$. The general form of the pitching moment about the leading edge is:

$$
C_{m, l e}=\left(-\frac{1}{2} \pi+E_{o}\right) \alpha+F_{o} \tau
$$

where $E_{o}$ and $F_{o}$ are coefficients that are functions of the blowing coefficient, very similar to the coefficients defined in the lift calculations. For the case of no blowing, the standard thin airfoil result is recovered. Good curve fits using data from Spence are:

$$
\begin{aligned}
& E_{o}=-0.3057 C_{j}^{1 / 2}-0.2466 C_{j}+0.0406 C_{j}^{3 / 2} \\
& F_{o}=-1.5868 C_{j}^{1 / 2}-0.6945 C_{j}-0.0437 C_{j}^{3 / 2}
\end{aligned}
$$

In the case of a trailing edge flap with a jet, the pitching moment's angle of attack relationship will remain the same, but the change due to the aft flap must be accounted for. A superposition of the classical flap contribution and the additional effects of the jet deflection leads to:

$$
C_{m, l e}=\left(-\frac{1}{2} \pi+E_{o}\right) \alpha-\left(\frac{1}{2} \chi+\sin (\chi)+\frac{1}{4} \sin (2 \chi)\right) \delta_{f}+G_{o} \delta_{f}
$$


where $G_{o}$ is a coefficient dependent on both $C_{j}$ and flap chord.

Finding a functional relation for $G_{o}$ was difficult due to the limited data provided by Spence. In addition, since he only provided pitching moment data up to a blowing coefficient of 5, one cannot use curve fits that significantly deviate for high blowing coefficients (such as high-order polynomials). It was originally hoped that a single functional relationship could be established for any flap chord, but that proved difficult. In addition, it would be largely unnecessary since the overwhelming majority of designs use flap chord ratios of 0.20 to 0.35 . Thus, the easiest approach for any practical design was to simply establish a good fit for $\mathrm{G}_{\mathrm{o}}$ based on Spence's data at a flap chord of 0.30:

$$
G_{o}=-0.3318 C_{\mu}^{1 / 2}-1.0332 C_{\mu}+0.0842 C_{\mu}^{3 / 2}
$$

At this point, the moment can be transferred to the quarter chord location as:

$$
C_{m, 0.25 c}=\frac{1}{4} C_{l}+C_{m, l e}
$$

This completes the current use of the jet-flap theory. Since it is based on 2-D thin airfoil theory (note that all the previous equations reduce to the classic formulas when there is no blowing) no drag force can be predicted from the theory. Drag will be discussed in detail later in this chapter.

\section{3 - Circular Streamline Theory}

Having a well-documented, analytical method for the jet-flap is a key step for powered-lift designs. However, as mentioned previously, the basis of classical jet-flap analysis is inviscid, thin-airfoil theory. The flow of the jet over the surface is not accounted for and this does introduce discrepancies into the analysis. A method should be devised to approximate the surface pressures induced on the curved surface wetted by 
the jet. The most precise way of doing this would include a complete analysis of a walljet flowing over a convex surface in the presence of a non-uniform external flow field. Such an analysis is not possible analytically, though some approximate work was done by Roberts $^{(39)}$.

Since only an approximation is needed, a simpler approach is proposed. We theorize that the nearly circular attached flow streamlines produced by coanda effect can be represented by potential flow, since that flowfield has circular streamlines and satisfies the boundary condition on the circular surface. A typical wall-jet velocity profile is shown in Figure 4-3.

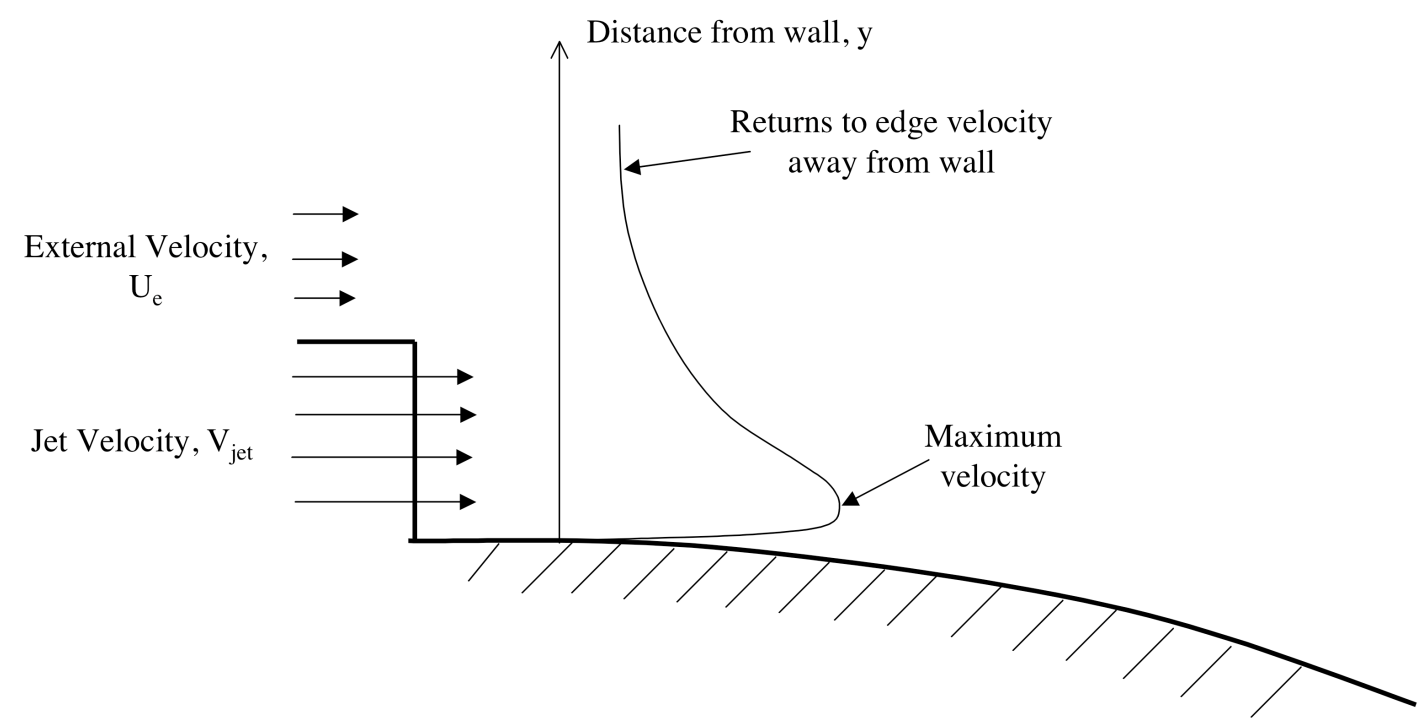

Figure 4-3: Example of a wall jet velocity profile

The decay of velocity in the outer jet layer looks like it could be modeled with a potential flow vortex. Consider the illustration given in Figure 4-4 showing the potential flow streamlines and velocity field induced by a point vortex. 


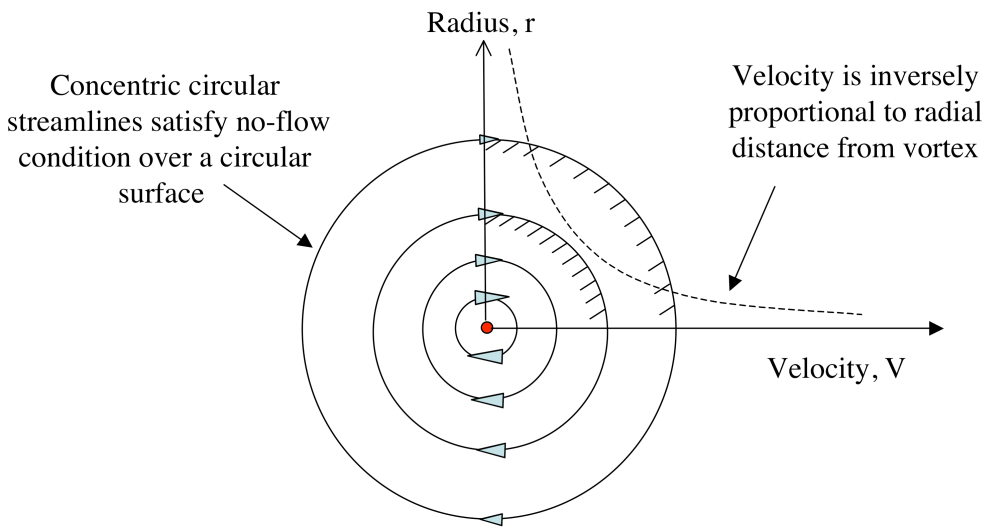

Figure 4-4: Streamlines and velocity profile produced by a point vortex

The thin boundary layer will contribute very little to the surface pressure (pressure is assumed constant across the boundary layer). This method would be relatively simple and could be applied without solving for the velocity of the entire flowfield. To check this model, figures will be presented showing comparisons with published surface pressure data from Davenport ${ }^{(24)}$ and $\operatorname{Sussman}^{(8)}$. Davenport, in fact, did include a small amount of work with Circular Streamline Theory in his analysis and demonstrated that circular streamline theory can provide reasonable estimates of the surface pressure.

\subsection{1 - Derivation of Circular Streamline Theory for USB}

First look at the jet in an ambient atmosphere case, i.e. a jet over a curved surface with no external freestream velocity. Starting with Bernoulli's equation for incompressible flow,

$$
p_{o}-p=1 / 2 \rho_{j e t} V^{2}
$$

where the velocity, $V$, is proportional to $1 / r$ for a vortex singularity. Writing the velocity in terms of this vortex strength, $\Gamma_{\mathrm{PV}}$, and then taking the derivative: 


$$
\begin{aligned}
& p_{o}-p=1 / 2 \rho_{j e t}\left(\frac{\Gamma_{P V}^{2}}{4 \pi^{2} r^{2}}\right) \\
& \frac{d p}{d r}=\rho_{j e t}\left(\frac{\Gamma_{P V}^{2}}{4 \pi^{2} r^{3}}\right)
\end{aligned}
$$

This ODE is integrable, so integrate $p$ from $p_{\text {wall }}$ to $p$, and $r$ from $R$ to $R+t$ where $t$ is the thickness of the jet. This gives:

$$
p-p_{\text {wall }}=\frac{-\rho_{j e t} \Gamma_{P V}^{2}}{2\left(4 \pi^{2}\right)}\left[\frac{1}{(R+t)^{2}}-\frac{1}{R^{2}}\right]
$$

Since $p$ at the edge of the jet is equal to the local static pressure, which is $p_{\infty}$, the LHS can be re-arranged to give the familiar quantity, $\Delta p$. The important remaining part is determining the appropriate value of the vortex strength, $\Gamma_{\mathrm{PV}}$. This quantity will be determined by satisfying the maximum jet velocity (which is known) at a given radius. Refer to this arbitrary location as the "reference" radius, $R_{r e f}$. Again, writing the vortex strength in terms of $V_{\text {exit }}$ and $r$ gives:

$$
\Delta p=\frac{\rho_{j e t} R_{r e f}^{2} V_{e x i t}^{2}}{2}\left[\frac{1}{(R+t)^{2}}-\frac{1}{R^{2}}\right]
$$

and for $C p$ based on the jet dynamic pressure this leads to:

$$
C_{p}=R_{r e f}^{2}\left[\frac{1}{(R+t)^{2}}-\frac{1}{R^{2}}\right]
$$

The approximation is clearly dependent on the radius used to find $\Gamma_{\mathrm{PV}}$. It is useful to examine this development compared to experimental surface pressures of wall jets flowing over curved surfaces. Figure 4-5 shows an experimental setup used by Davenport $^{(24)}$ to measure the surface pressures induced over a curved USB flap. 


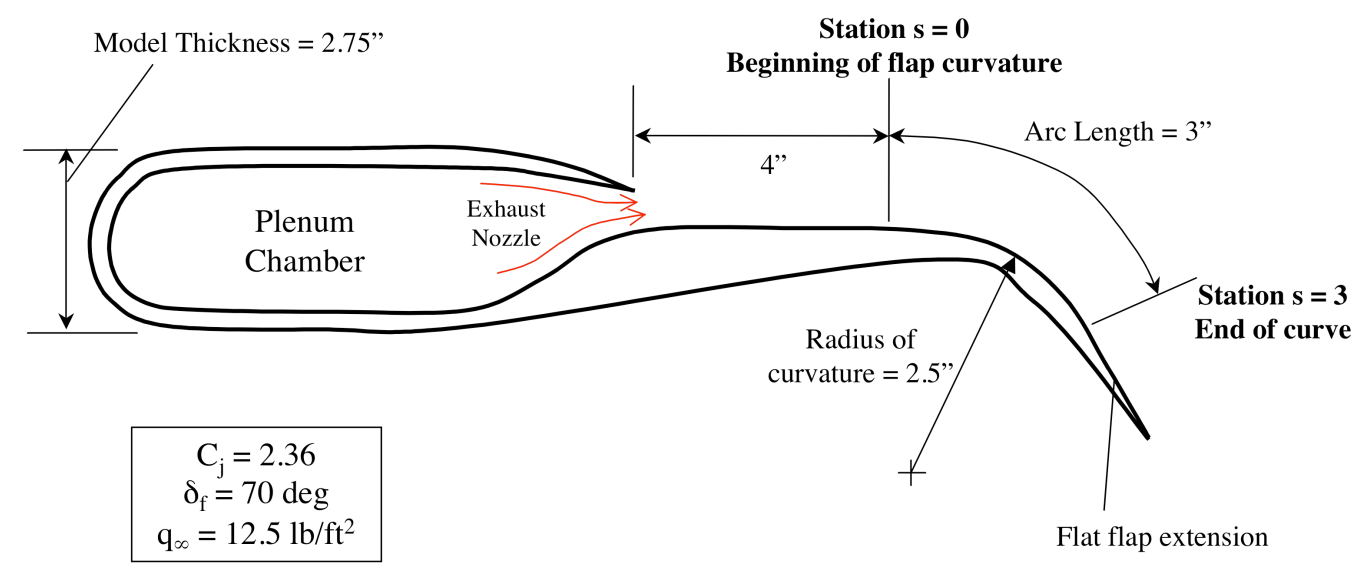

Figure 4-5: Sketch of experimental setup used in Ref. 24

Using the experimental data provided in reference 24, circular streamline theory predictions of equation (4-20) can be compared with different values of $R_{r e f}$. Note that $y_{m}$ refers to the distance from the wall where the maximum velocity occurs in the profile.

Figure 4-6 shows these comparisons.

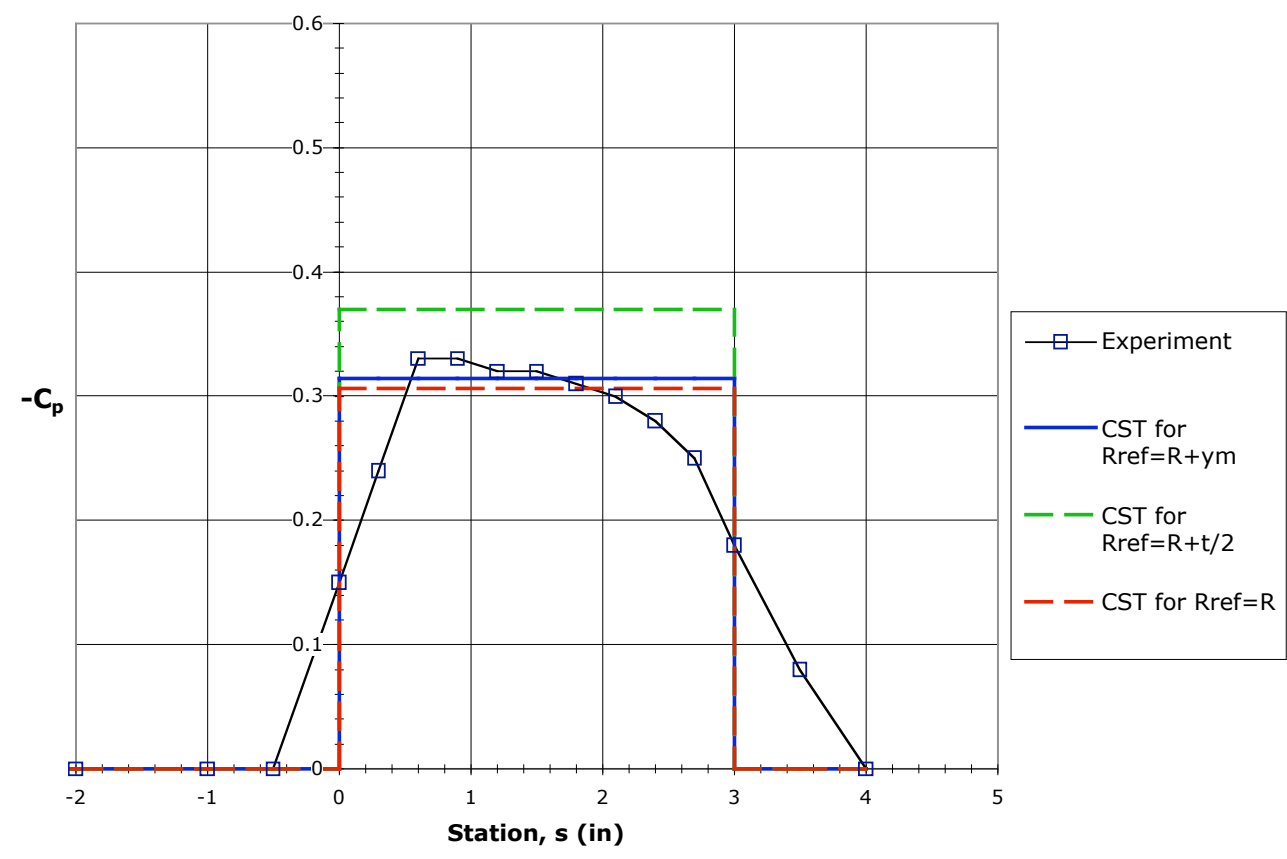

Figure 4-6: Comparison of CST with data from Ref 24 (no external flow)

Looking at the area under the curves, using $R_{r e f}$ of $R+y_{m}$ appears to be the best choice for an ambient atmosphere case, estimating the area to within 3.5\%. However, all of the 
curves approximate the area within $10 \%$ of the actual value, meaning that any choice for $R_{r e f}$ less than or equal to the jet half-width should be sufficient. This is important since an analytical expression for $y_{m}$ will not be available.

Getting a good approximation for the performance with an external stream is clearly more important for USB applications, but unfortunately it is also more complicated. The performance can be significantly affected by altering the external flow (even though the jet dynamic pressure may be $\sim 100$ times that of the free stream). The presence of the external flow changes the jet velocity profiles and behavior. As discussed in Chapter 2, the pressure field outside the jet will produce acceleration of the jet flow around the surface (to jet velocities significantly greater than the exit velocity)

To model the jet at any given surface station, introduce a uniform velocity potential to go with our point vortex as illustrated in Figure 4-7.

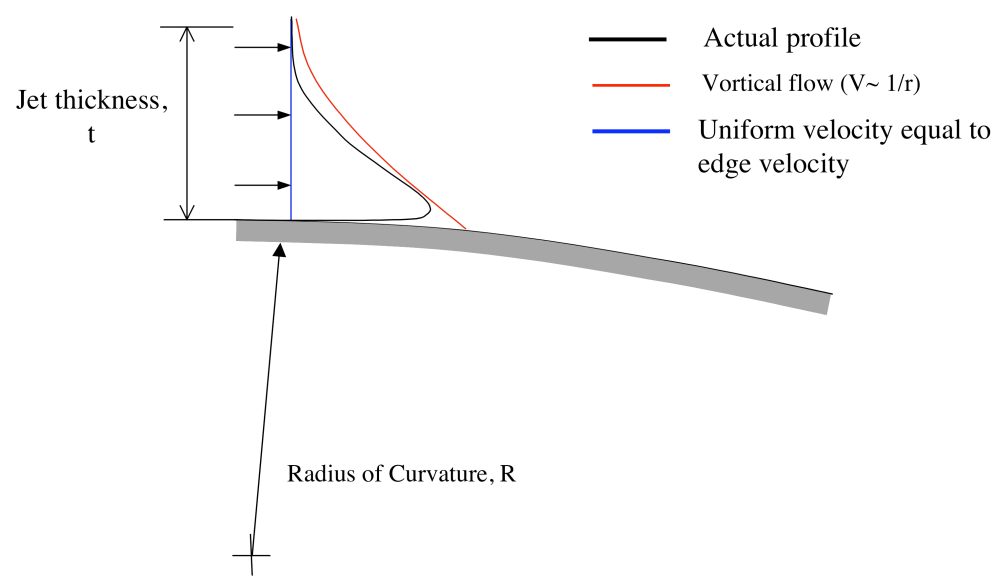

Figure 4-7: Sketch of jet velocity profiles with an external flow

From potential flow theory, the velocity is then:

$$
V=U_{e}(x)-\frac{\Gamma_{P V}}{2 \pi r}
$$

Following the same procedure as before: 


$$
\frac{d p}{d r}=\rho_{j e t}\left(\frac{\Gamma_{P V}^{2}}{4 \pi^{2} r^{3}}-\frac{\Gamma_{P V} U_{e}}{2 \pi r^{2}}\right)
$$

which is integrable. Integrating and re-arranging gives,

$$
p_{w}-p_{\text {localstatic }}=\frac{\rho_{j e t} \Gamma_{P V}^{2}}{2\left(4 \pi^{2}\right)}\left(\frac{1}{(R+t)^{2}}-\frac{1}{R^{2}}\right)-\frac{\rho_{j e t} \Gamma_{P V} U_{e}}{2 \pi}\left(\frac{1}{R+t}-\frac{1}{R}\right)
$$

Before starting to determine the vortex strength, $\Gamma_{\mathrm{PV}}$, note a few things that will have a large effect on the results. First, there is no way to accurately describe the acceleration of the jet due to the external pressure field. Tied to that, the external edge velocity, $U_{e}$, can change substantially throughout the flowfield. Thus, the profiles are not self-similar. (This condition is important to note because self-similarity would allow analytic models such as Roberts ${ }^{{ }^{(40)}}$ to be used to predict the wall jet behavior and induced surface pressures) We can find the edge condition at the jet exit however. For now, assume that the jet maximum velocity is at some average condition over the curve, say $n V_{\text {exit }}$, where $n$ is a scalar of $\mathrm{O}(1)$ which must somehow be tied to the external pressure field.

Using the definition of $\Gamma_{\mathrm{PV}}$ solved for $V_{\text {exit }}$ at $R_{\text {ref }}$ :

$$
\begin{aligned}
p_{w}-p_{\text {static }} & =\frac{\rho_{\text {jet }}}{2} R_{\text {ref }}^{2}\left(U_{e}-n V_{\text {exit }}\right)^{2}\left(\frac{1}{(R+t)^{2}}-\frac{1}{R^{2}}\right) \\
& -\rho_{\text {jet }} R_{\text {ref }} U_{e}\left(U_{e}-n V_{\text {exit }}\right)\left(\frac{1}{R+t}-\frac{1}{R}\right)
\end{aligned}
$$

Re-arranging this to get a delta pressure on the LHS, then normalizing by the jet dynamic pressure to get a pressure coefficient, we obtain the final 2 equations: 


$$
\begin{aligned}
& \Delta p=\left(p_{\text {local }}-p_{\infty}\right)+\frac{\rho_{\text {static }}}{2} R_{\text {ref }}^{2}\left(U_{e}-n V_{\text {exit }}\right)^{2}\left(\frac{1}{(R+t)^{2}}-\frac{1}{R^{2}}\right) \\
& -\rho_{j e t} R_{r e f} U_{e}\left(U_{e}-n V_{e x i t}\right)\left(\frac{1}{R+t}-\frac{1}{R}\right) \\
& C_{p}=\left(\frac{q_{\infty}}{q_{\text {jet }}}\right) C_{p, e d g e}+R_{r e f}^{2}\left(\frac{U_{e}}{V_{\text {exit }}}-n\right)^{2}\left(\frac{1}{(R+t)^{2}}-\frac{1}{R^{2}}\right) \\
& -2 R_{\text {ref }}\left(\left(\frac{U_{e}}{V_{\text {exit }}}\right)^{2}-n \frac{U_{e}}{V_{\text {exit }}}\right)\left(\frac{1}{R+t}-\frac{1}{R}\right)
\end{aligned}
$$

Note that equations (4-26) and (4-27) reduce to equations (4-20) and (4-21), respectively, when the freestream velocity goes to zero.

Predictions from these equations can be compared with Davenport's data. To establish a comparison, estimate the local static pressure/local edge velocity from the thickness of his experimental model, shown in Figure 4-5. For a $q_{\infty}$ of $12.5 \mathrm{lb} / \mathrm{ft}^{2}$, this edge velocity is around $125.6 \mathrm{ft} / \mathrm{s}$. Take the reference radius, $R_{\text {ref }}$, to be $R+t / 2$ since this seems to correlate the data well (see Fig 4-5), and choose $n$ to be 1.25. This means that the jet is accelerated by about $25 \%$ around the curve by the external pressure field. This number was used based on experimental velocity profiles taken from Davenport. Any other numbers remain identical from previous computations and were taken directly from his paper. The pressure along the curve is shown in Figure 4-7. Note that the surface pressures are approximated well, and better agreement could be expected with better information about the jet conditions. 


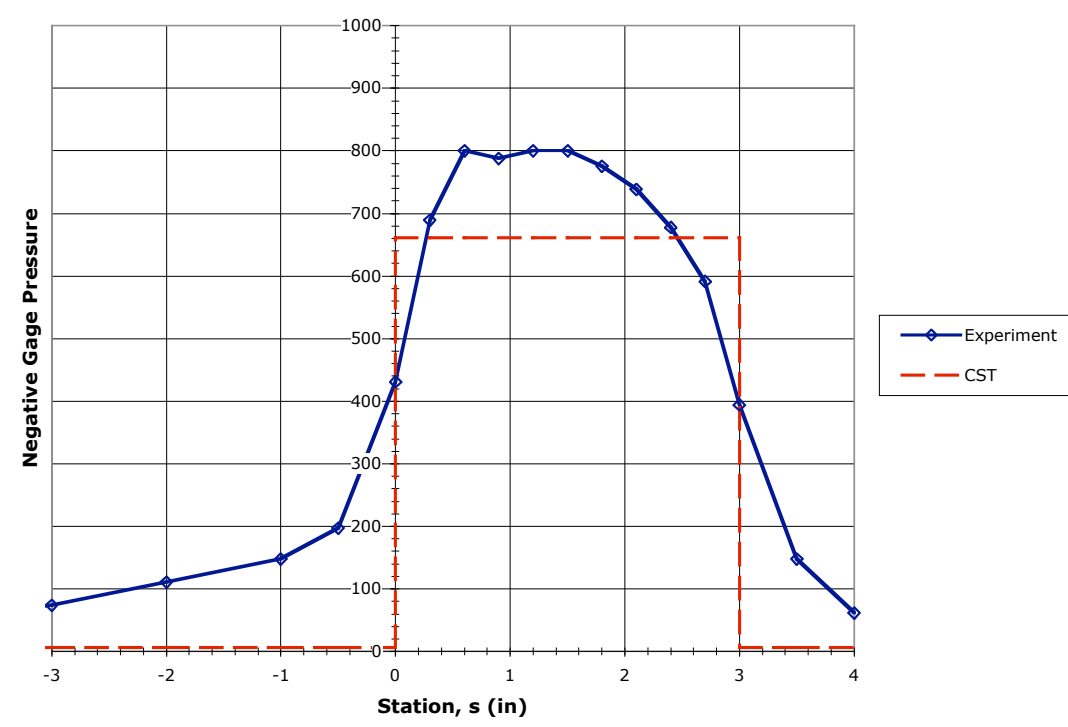

Figure 4-8: Comparison of CST with data from Ref 23 with an external flow

Comparison was also done with data published by Sussman ${ }^{(8)}$ from the YC-14 flight test program. Although the geometry data isn't presented well in this paper, some data can be inferred from knowledge of the YC-14. Figure 4-9 shows the ground test data gathered and the predictions from the Circular Streamline Theory. Although the geometry is roughly estimated, the results are encouraging.

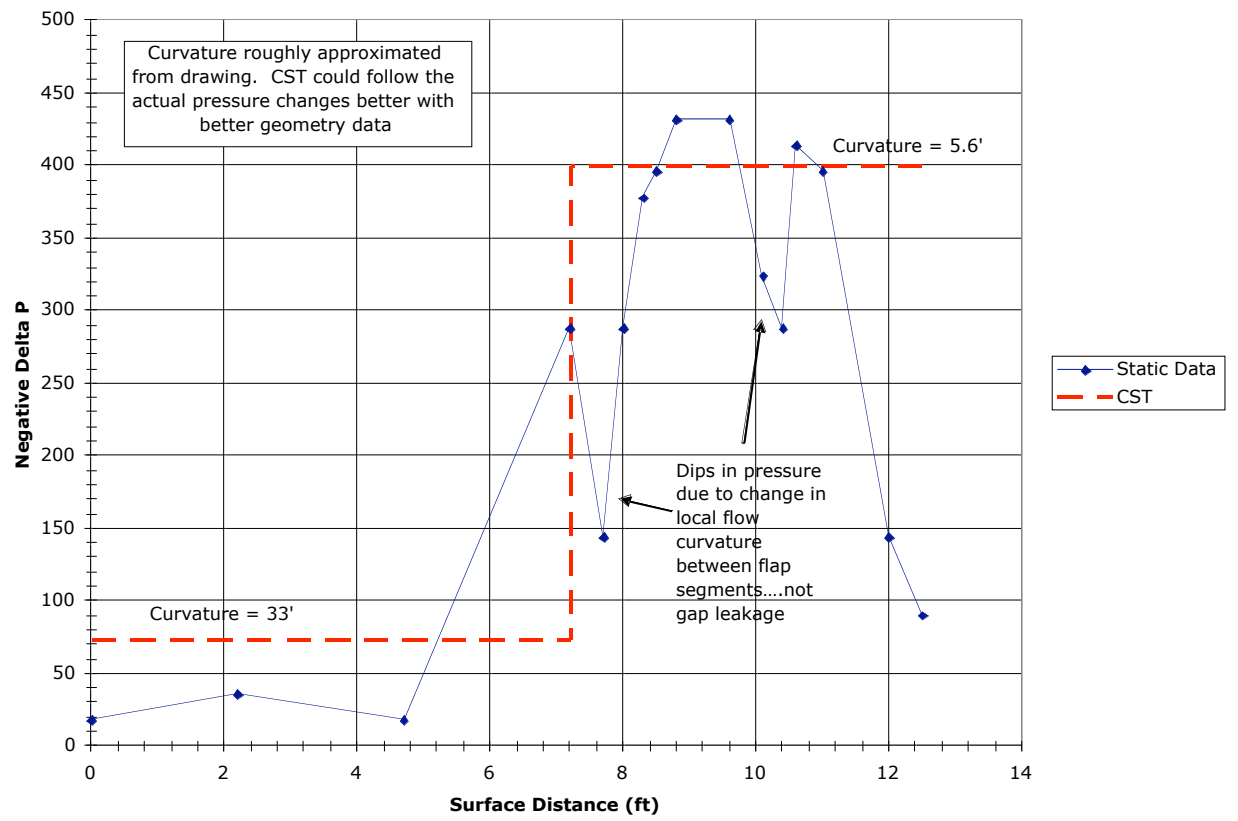

Figure 4-9: Comparison of CST with static test data from Ref. 8 
Pressure data was also taken on the USB flap surface along the engine centerline during flight. For an airspeed of $210 \mathrm{ft} / \mathrm{s}$, jet exit velocity of $1200 \mathrm{ft} / \mathrm{s}$, and a nozzle height of $1.5 \mathrm{ft}$, Figure 4-10 was generated using equation (4-26).

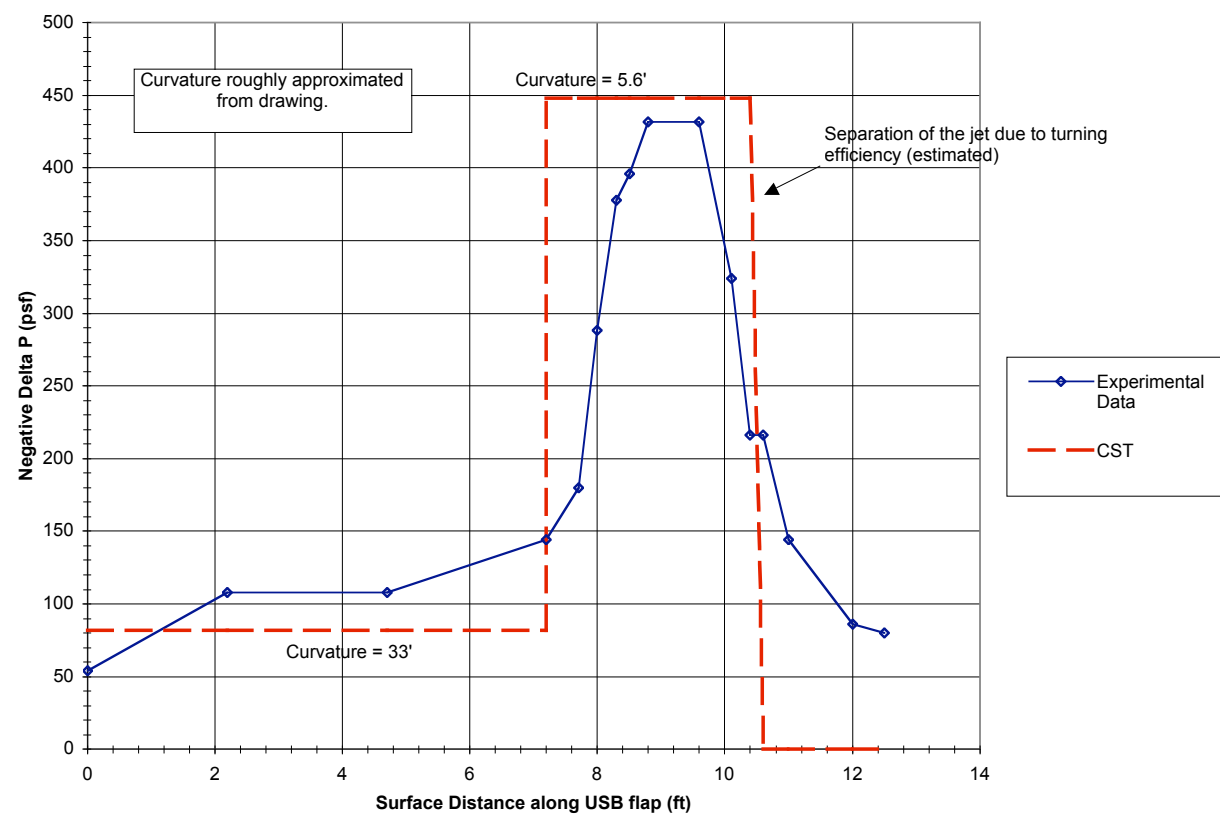

Figure 4-10: Comparison of CST with flight test data from Ref. 8

\subsection{2 - Lift, Drag, and Pitching Moment calculations}

To calculate forces due to these pressures, either continuous functions for the surface geometry must be established or the surface must be discretized into "N" small segments over which the pressure is assumed constant. Since we will be dealing with many calculations and the surface geometry can change, the latter option is more useful. A representation of this idea is shown in Figure 4-11: 


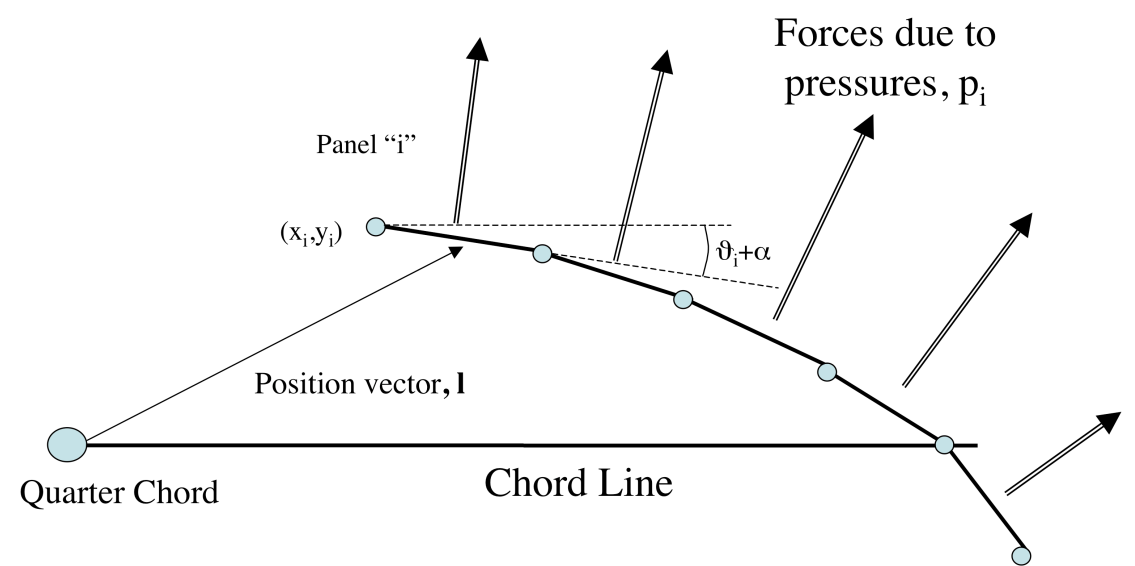

Figure 4-11: Drawing of a USB surface discretized into small segments

Each segment is picked to be the same length, but it will have a different orientation relative to the aerodynamic center. The pressure that develops over a given panel is directly related to the local radius of curvature. The local radius can be found from calculus using finite differencing of the panel coordinates.

The direction of the pressure force is the surface normal vector, given as:

$$
\mathbf{N}_{i}=\cos \theta_{i} \mathbf{i}+\sin \theta_{i} \mathbf{j}
$$

It follows then that the force in the $x$ and $y$ directions for each panel consist of the induced pressure on the panel times the panel length, $\mathrm{s}_{\mathrm{i}}$ :

$$
\begin{aligned}
& f_{x, i}=p_{i} s_{i} \cos \theta_{i} \\
& f_{y, i}=p_{i} s_{i} \sin \theta_{i}
\end{aligned}
$$

The total force in the $x$ or $y$ direction is then the sum of forces developed on each panel. Taking the summation and normalizing to a 2-D coefficient gives a lift and drag increment due to the induced surface pressure:

$$
\begin{aligned}
\Delta C_{l} & =\left(\frac{1}{q_{\infty} c}\right) \sum_{i=1}^{N} p_{i} s_{i} \cos \theta_{i} \\
\Delta C_{d} & =\left(\frac{1}{q_{\infty} c}\right) \sum_{i=1}^{N} p_{i} s_{i} \sin \theta_{i}
\end{aligned}
$$


Similarly, the moment about the aerodynamic center created by this pressure is simply the cross product of the force vector on the panel and the position vector, $\mathbf{l}$.

$$
\Delta C_{m, a c}=\frac{1}{q_{\infty} c^{2}}(\mathbf{F} \times \mathbf{l})
$$

Taking the equivalent force to act at the midpoint of each panel, $\left(\mathrm{x}_{\mathrm{m}, \mathrm{i}}, \mathrm{y}_{\mathrm{m}, \mathrm{i}}\right)$ and the aerodynamic center to be at approximately the quarter-chord, the position vector becomes:

$$
\mathbf{l}_{i}=\left(x_{m, i}-0.25 c\right) \mathbf{i}+\left(y_{m, i}\right) \mathbf{j}
$$

The increment in pitching moment then becomes:

$$
\Delta C_{m, 0.25 c}=\frac{1}{q_{\infty} c^{2}} \sum_{i=1}^{N} p_{i} s_{i}\left(y_{m, i} \sin \left(\theta_{i}\right)-\left(x_{m, i}-0.25 c\right) \cos \left(\theta_{i}\right)\right)
$$

Thus the lift, drag, and pitching moment increments due to induced surface pressures can be approximated given the surface geometry and the flow conditions.

\section{4 - Drag Estimation}

In two-dimensions, the drag sources present for a subsonic airfoil are the skin friction drag and the pressure drag. Pressure drag for a typical airfoil comes from separated flow, which is usually not an issue when blowing is used. As seen in the previous section, however, there is a pressure force from the jet that acts as drag. The remaining component is skin friction, which can be estimated in the standard manner using a form factors approach with empirical data.

The skin friction is accounted for using a form factors approach similar to that presented by Mason ${ }^{(15)}$ and Torenbeek ${ }^{(41)}$. In this case the portion of the surface wetted by 
the jet has a different skin friction coefficient. The friction drag coefficient (part of $\mathrm{C}_{\mathrm{do}}$ ) is:

$$
C_{d}=\left(C_{F, f_{s}}\left(\frac{S_{f s}}{S_{r e f}}\right)+C_{F, j e t}\left(\frac{S_{j e t}}{S_{r e f}}\right)\right) F F
$$

where " $f s$ " denotes normal flow and "jet" denotes the area exposed to the exhaust, and $F F$ represents the form factor of our airfoil. The skin friction coefficients are given by the Prandtl-Schlichting formula:

$$
C_{F}=\frac{0.455}{\left(\log _{10}\left(\operatorname{Re}_{c}\right)\right)^{2.58}}
$$

The form factor used is:

$$
F F=1+1.8(t / c)+50(t / c)^{4}
$$

For the pressure drag, a relation was established in equation (4-30) above. However, as described in Chapter 2, not all of this drag will be realized. In fact, a large portion of it can and will be balanced by leading edge suction. A simple multiplier can be applied to equation (4-30) in that respect. Call this multiplier the "pressure drag factor" and denote it by the variable, $\zeta$. The determination of the magnitude of this multiplier is discussed in the next chapter. Note that for the real-world situation, there can also exist a significant form drag with no blowing. This quantity is not accounted for in this method.

\section{5 - Entrainment Effects}

The effects of entrainment are more difficult to quantify. At this point, there is no established method for incorporating these types of effects for upper surface blown configurations. The goal of this work is to provide enough empirical approximations of these effects to correlate existing data. A set of experiments designed to measure 
entrainment effects within the framework of the current prediction method would be the next logical step, but that has not been done here.

Basically, the lift augmentation produced by direct fluid entrainment from the jet is needed. For small amounts of blowing, we should also account for the positive lift and drag effects produced by boundary layer control. Following the breakdown shown in Chapter 2, a multiplicative factor is used on the lift to account for entrainment effects. Call this factor, $\eta_{e n t}$. A full discussion of the development of this and other factors is given in the following chapter. While the choice of these factors can be subjective and/or sensitive to configuration, it will be shown that good correlation can be obtained via relatively simple empirical relations.

\section{6 - Total Two-Dimensional Equations for Lift, Drag, and Pitching Moment}

Having developed the constituent pieces of the method, we can now present the total equations for 2-D upper surface blowing performance characteristics. These equations require only the specification of the jet exhaust conditions, nozzle parameters, surface geometry, and freestream conditions. They provide estimations of the sectional lift, drag, and pitching moment coefficients.

\subsection{1 - Lift Coefficient}

The 2-D lift coefficient is given by a combination of equations (4-9) and (4-29) and the entrainment factor described above:

$$
C_{l}=\left(1+\eta_{\text {ent }}\right)\left(\begin{array}{c}
\left(2 \pi \alpha+4 \pi B_{o} \alpha+2\left(\chi+\sin \chi+2 \pi D_{o}\right) \delta_{f}+4 \pi \gamma\left(1+C_{o}\right)\right) \\
+\left(\frac{1}{q_{\infty} c}\right) \sum_{i=1}^{N} p_{i} s_{i} \cos \theta_{i}
\end{array}\right)
$$


where all the quantities have been defined previously. The functional dependence can be written as:

$$
C_{l}=f\left(\alpha, \gamma, E, \delta_{f}, C_{\mu}, \operatorname{Re}_{c}, \operatorname{Re}_{j e t}, \text { nozzle geometry }\right)
$$

where $\operatorname{Re}_{c}$ and $\mathrm{Re}_{\text {jet }}$ are the Reynolds numbers of the freestream and jet flow respectively.

\subsection{2 - Pitching Moment Coefficient}

The 2-D pitching moment coefficient is based on a combination of equations (4-

13),(4-15), and (4-34) above.

$$
\begin{aligned}
C_{m, 0.25 c}= & \left(\frac{1}{4} C_{l, \text { JetFlap }}+\left(-\frac{1}{2} \pi \alpha+E_{o} \alpha\right)-\left(\frac{1}{2} \chi+\sin \chi+\frac{1}{4} \sin (2 \chi)\right) \delta_{f}+G_{o} \delta_{f}\right)+ \\
& \frac{1}{q_{\infty} c^{2}} \sum_{i=1}^{N} p_{i} s_{i}\left(y_{m, i} \sin \left(\theta_{i}\right)-\left(x_{m, i}-0.25 c\right) \cos \left(\theta_{i}\right)\right)
\end{aligned}
$$

This equation has the same functional dependence as the lift coefficient.

\subsection{3 - Drag Coefficient}

The 2-D drag coefficient is given by a combination of the equations (4-31), (4-35) and the pressure drag factor.

$$
C_{d}=\left(C_{F, f s}\left(\frac{S_{f s}}{S_{r e f}}\right)+C_{F, j e t}\left(\frac{S_{j e t}}{S_{r e f}}\right)\right) * F F+\left(\zeta\left(\frac{1}{q_{\infty} c} \sum_{i=1}^{N} p_{i} s_{i} \sin \theta_{i}\right)\right)
$$

where, again, $S$ represents a surface length around the airfoil. The functional dependence for the drag coefficient is very similar to the lift and pitching moment coefficients but includes a dependence on the airfoil thickness, $t / c$. 


\section{Chapter 5: Correlation of 2-D method with Experimental Data}

This chapter documents the correlation of the methodology outlined in the previous chapter with published experimental data for 2-D USB configurations. The data used was primarily taken from a series of experiments performed at the University of Texas at Arlington ${ }^{(42),(43),(44)}$. These experiments employed a modified, symmetric NACA airfoil with a propulsive nozzle located on the aft portion of the upper surface. The lift and pitching moments were recorded for various angles of attack and blowing coefficients. The development of empirical relations for various factors is shown, and these factors are seen to provide good correlation over a physically realistic range of blowing coefficients.

\section{1 - Description of Published Experiments}

\subsection{1 - History and Overview}

The propulsive wing concept was an ongoing research activity at the Aerodynamics Research Center at the University of Texas at Arlington during the 1980s. These projects were overseen by Dr. Don Wilson, with primary research being done by Roberts ${ }^{(42)}$, 
Pernice $^{(43)}$, and Jeon ${ }^{(44)}$. The main purpose of this work was to provide two-dimensional force and moment data for a propulsive wing system. A small-scale wing was built which used compressed air exhausting over the aft portion of the upper surface to simulate the engine flow. All researchers used modified NACA sections, and all were symmetric with the exception of Roberts ${ }^{(42)}$. Figure 5-1 shows the test article:

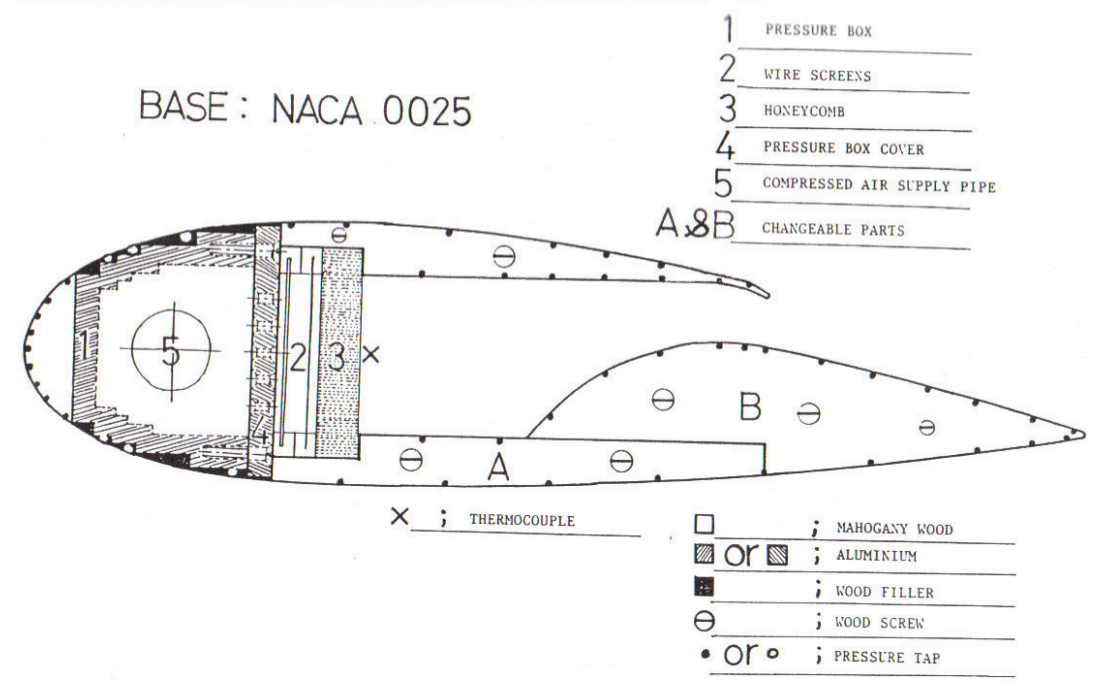

Figure 5-1: Propulsive wing section used in experiments by Jeon ${ }^{(44)}$

Pressure taps were located at the mid-span of the wing spaced around the full contour of the section. Measurements were taken to ensure that the flow was sufficiently twodimensional at this location, given the small span $(b<1 \mathrm{ft})$ of the models involved. Quadrature was used to get force and moment measurements from the pressure data. Jeon also attempted to make drag measurements from the wake rake data. Since the forces and moments were found from an integration of surface pressures, the jet reaction lift is not included in the test data, nor is the skin friction drag. These omissions were accounted for in the current calculations. 
In addition to collecting force and moment data, much of the research involved the search for correlation parameters for USB configurations. The goal was to find a parameter or parameter grouping which correlated the lift coefficient linearly. It was found that the velocity ratio between the jet and the external stream at the jet exit provided a roughly linear correlation. However, this ratio is not very useful in a design sense since the external velocity requires some work to obtain. The study of this type of correlation parameter did, however, bring about a very useful study of the entrainment ability of the exhaust jet. This information was helpful in developing empirical relations in the current study.

The blowing coefficient, $C_{j}$, used in this work has a non-linear relationship with the lift, as does the net thrust coefficient. (Recall that for a 2-D case, the blowing coefficient is equivalent to the gross thrust coefficient) The non-linearity of $C_{l}$ with $C_{j}$ is evident in the equations from jet-flap theory. In addition, Pernice ${ }^{(43)}$ demonstrated that the blowing coefficient provides a poor correlation of the data in cases where the jet velocity and freestream velocity are roughly the same magnitude. However, such cases represent infeasible designs because this condition indicates the propulsive system has zero net thrust. The blowing coefficient provides a familiar, useful tie to the propulsion system making it the most efficient correlation parameter for conceptual design despite the nonlinearity.

One drawback to the use of these experiments is that no work was done incorporating deflected trailing edge flaps. It is expected that the presence of the flaps (if properly contoured) would greatly increase the force and pitching moments. Flaps essentially provide an increase in trailing edge flow angle and aft surface curvature. Their presence 
would also alter the external flow. However, it is believed that if good correlation can be achieved with the current experimental data, then the effects of a deflected flap can be estimated using basic flow principles. Flap effects were accounted for in all 3-D results presented later in this work.

\subsection{2 - Experimental Data}

Two-dimensional force and pitching moment data were recorded over a wide range of blowing coefficients, angles of attack, and dynamic pressures. The early experiments done by Roberts ${ }^{(42)}$ were less complete than the later experiments by Pernice ${ }^{(43)}$ and $\mathrm{Jeon}^{(44)}$, thus the latter data was used much more for correlation. Drag data was presented by each researcher, but very little discussion or analysis was presented.

At this point, it is necessary to define what data was used to correlate the current method. Consider again the 2-D blowing coefficient:

$$
C_{j}=\frac{\dot{m} V_{e x i t}}{q_{\infty} c}
$$

where as with any 2-D coefficient, it is taken as "per unit span". Substituting the equation for dynamic pressure and equating the mass flow to the product of density, exit area, and exit velocity gives:

$$
C_{j}=\frac{\rho A_{\text {exit }} V_{\text {exit }}^{2}}{1 / 2 \rho V_{\infty}^{2} c} \propto\left(\frac{V_{\text {exit }}}{V_{\infty}}\right)^{2}
$$

Thus the blowing coefficient is proportional to the ratio of the exit velocity to the freestream velocity. Since most designs will employ turbofan engines, and the flight regime for high-lift USB operation is around 0.0-0.2 Mach, the vast majority of design 
blowing coefficients will be in the range of 0 to $\sim 10$. For this reason, the upper limit of the current correlation was set to be $C_{j}=10$ to match the upper limit of jet-flap theory.

The following plots from Pernice and Jeon show compiled data over the relevant range of blowing coefficients. The drag data is from Jeon only, since the previous data demonstrated correct trends but with unreasonable magnitudes (which was pointed out by the authors). The lift coefficient for two angles of attack is shown in Figure 5-2.

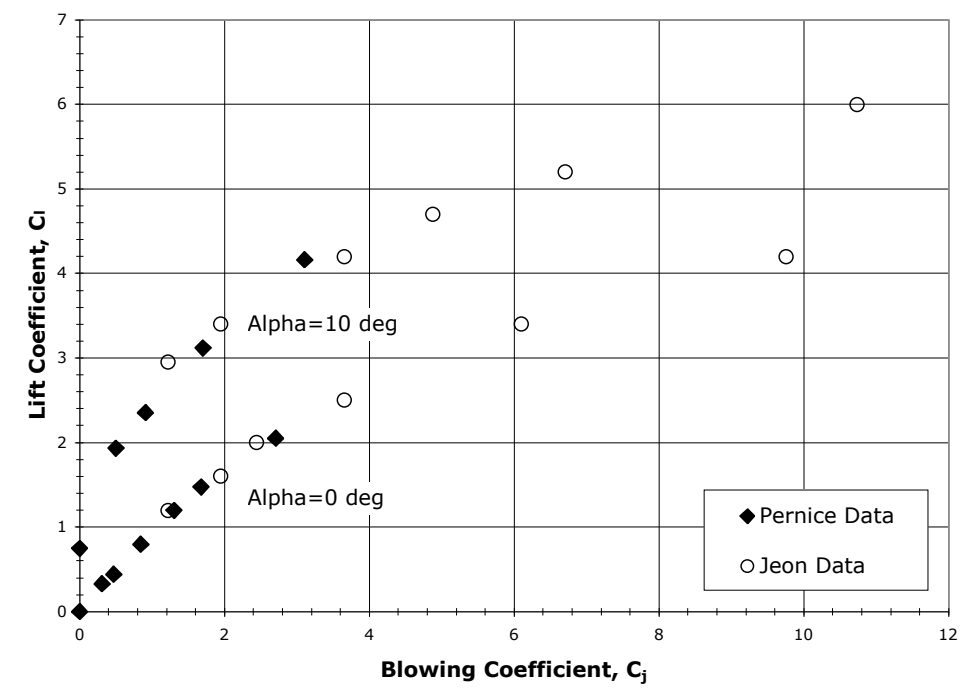

Figure 5-2: Sample of $C_{l}$ vs. blowing coefficient data from Pernice and Jeon And similarly, Figure 5-3 shows the pitching moment for two angles of attack:

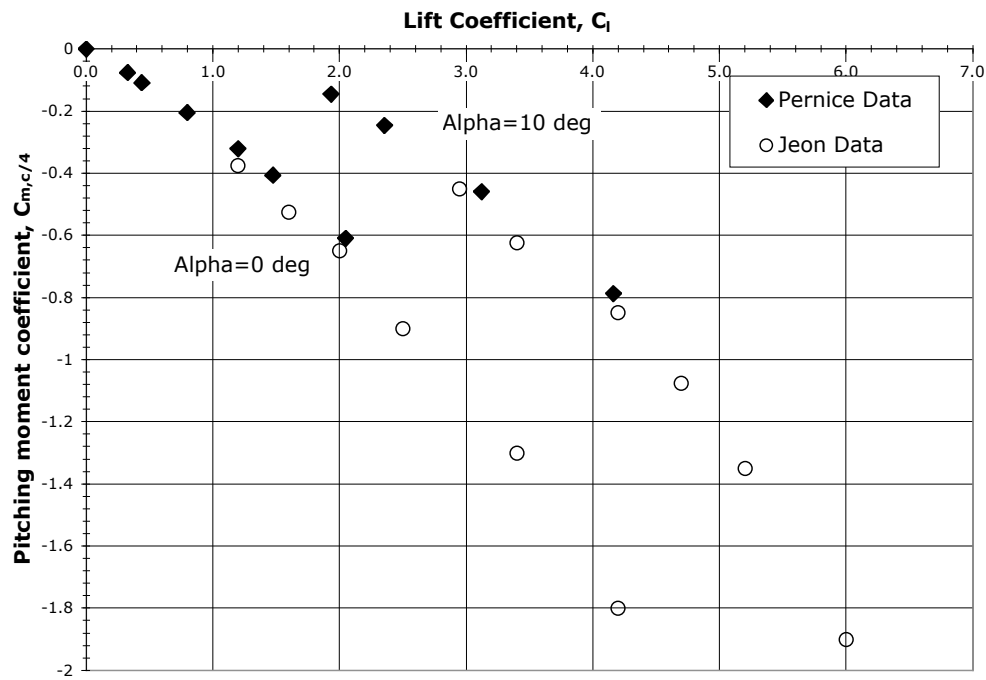

Figure 5-3: Sample of $C_{m, c / 4}$ vs. $C_{l}$ data from Pernice and Jeon 
Lastly, the drag data from Jeon is seen in Figure 5-4. Note that the lines are shown just to keep the data sets separate. Again, due to experimental limitations, these values represent only the pressure drag and not the skin friction drag.

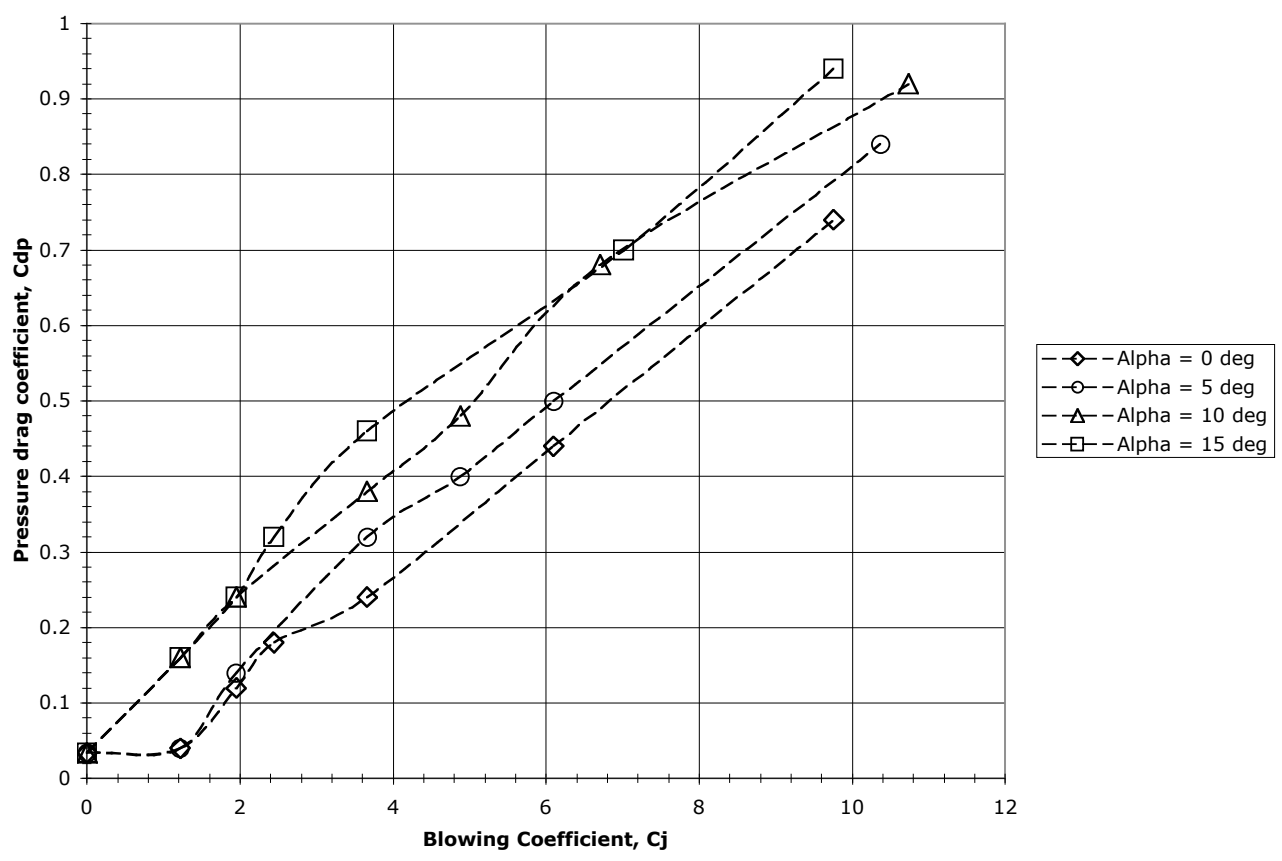

Figure 5-4: Test data for pressure drag vs. blowing coefficient from Jeon

\section{2 - Empirical factors and their physical basis}

There were three primary factors introduced through the derivation of equations in the previous chapter. These factors need to be developed to help match this method to the test data, and they should be based on established theory or published test data. The following developments are not, nor are they intended to be, complete or exact for all USB configurations. Additional experimental work could provide more detailed relations. The current ones are intended to show that relatively simple relations to approximate the physical processes can correlate the present method well. 


\subsection{1 - The $n$ factor}

This factor comes from the Circular Streamline Theory. Recall that there are low pressures directly induced by the curved wall jet flowing over the aft surface. An approximate equation for these pressures was derived in Chapter 4, equation (4-26). It is shown again here, ignoring the relatively small difference between freestream pressure and the local static near the jet exit:

$$
p=\frac{\rho_{\text {jet }}}{2} R_{r e f}^{2}\left(U_{e}-n V_{\text {exit }}\right)^{2}\left(\frac{1}{(R+t)^{2}}-\frac{1}{R^{2}}\right)-\rho_{\text {jet }} R_{r e f} U_{e}\left(U_{e}-n V_{\text {exit }}\right)\left(\frac{1}{R+t}-\frac{1}{R}\right)
$$

Here $n$ is a "jet acceleration" factor. Essentially, this factor relates the behavior of the jet and the freestream conditions. This factor allows slight adjustment of the exit condition in an average sense to account for the external flow.

Davenport ${ }^{(24)}$ says, "A considerable increase in jet velocity over the value at the nozzle exit is seen as the jet rounds a corner, especially at forward speeds where the jet is expanding into the low pressure region created by the outside flow field". The only data he presented showed a maximum increase of about 25 to $30 \%$ in jet velocity with a deflected flap. One can expect that this factor is a strong function of angle of attack and flap deflection. This factor will increase the force by decreasing the aft pressure, but it should not be allowed to become large, since this would make the pitching moments become too large.

With this in mind, a simple linear relation is suggested for the " $n$ " factor:

$$
n=1.0+\left(\frac{\alpha(\mathrm{deg})}{100}\right)+\left(\frac{\delta_{f}(\mathrm{deg})}{500}\right)
$$


which corresponds to values around 1 to 1.45 in the typical operational range of a USB configuration. Note also that this allows $n$ to be negative for negative angles of attack. This would correspond to the external pressure field slowing down the jet flow.

\subsection{2 - The $\eta_{\text {ent }}$ factor}

This factor is a measure of the effects of direct fluid entrainment. It is harder to quantify, but has been studied. Pernice ${ }^{(43)}$ deals with this in some detail. The highvelocity jet will entrain fluid creating a favorable pressure gradient (or greatly relieve an adverse gradient) forward of the nozzle. Pernice indicates that for a $100 \mathrm{ft} / \mathrm{s}$ increase in jet velocity, the fluid just forward of the exit can increase by $\sim 15 \mathrm{ft} / \mathrm{s}$ with all other variables held constant. Thus, the pressure distribution is improved and lift is gained. Figure 5-5, similar to those presented by Korbacher ${ }^{(17)}$ and Gainer ${ }^{(37)}$, demonstrates this qualitatively:

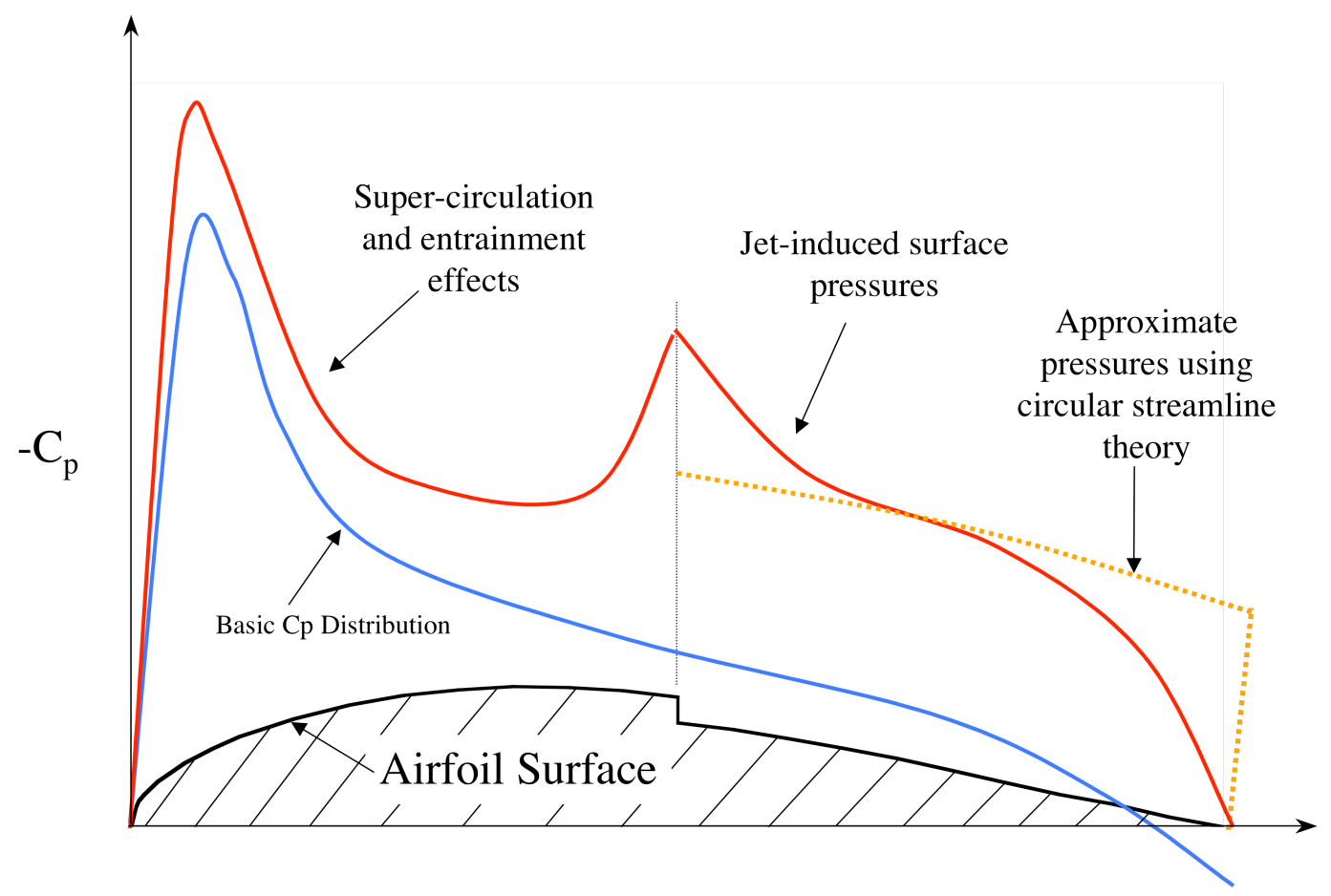

Figure 5-5: Pressure Coefficients due to blowing effects 
It is expected that this effect is primarily a function of the blowing coefficient. Recall from Chapter 4 that the entrainment factor modifies the total lift coefficient as:

$$
C_{l}=\left(1+\eta_{e n t}\right)\left(C_{l, \text { JetFlap }}+C_{l, \text { Jet Pressure }}\right)
$$

In general, (and certainly for this data) this effect will not contribute significantly to the pitching moment. Based upon the fact that Pernice found a linear relation between the effective velocity ratio $\left(\mathrm{V}_{\text {jet,exit }} / \mathrm{V}_{\text {external,exit }}\right)$ and the lift, the following function is initially suggested:

$$
\eta_{e n t}=0.05 C_{j}
$$

However, this factor can also be used to account for boundary layer control effects (BLC). These effects were described by Korbacher ${ }^{(17)}$. Essentially, these are the strong initial effects of blowing. For small blowing coefficients, the primary effect is to relieve adverse pressure gradients and eliminate separation. This is the steep increase in lift at small blowing coefficients seen in the sample data of Figure 5-2. Also, as the angle of attack increases, this initial slope increases. Consider the BLC effect to be allowing the airfoil to reach its potential since adverse gradients/separation become more prevalent for an airfoil with increasing angle of attack. The curves in Figure 5-2 could be approximately described by 2 linear functions. This idea is supported by work done by Roberts $^{(42)}$, who suggested a piecewise-linear fit to the data with the slopes/intercepts being determined empirically.

From a physical standpoint and also a programming standpoint, it makes sense to roll this effect into the "entrainment factor". For a small region of low blowing coefficients (say $C_{j}<0.8$ ), a multiplicative factor, say $\lambda_{B L C}$, is added to $\eta_{e n t}$ to reflect the sharp increase in lift capability. Thus, equation (5-6) becomes: 


$$
\eta_{e n t}=\left(0.05 C_{j}\right)\left(\lambda_{B L C}\right)
$$

Beyond these low blowing coefficients, the entrainment factor importance should decrease in a continuous fashion. This decrease corresponds to the decrease in slope for higher angles of attack.

The following linear function of angle of attack appears to be a reasonable empirical relationship for the BLC factor:

$$
\lambda_{B L C}=1+(0.4 \alpha)
$$

A good empirical fit for the variation of the entrainment factor beyond the initial region was found to be:

$$
\frac{d \eta_{\text {ent }}}{d C_{j}} \approx(-0.062-0.0008 \alpha(\mathrm{deg})) C_{j}
$$

for $C_{j}>0.8$. Thus, the entrainment factor is now a function of both angle of attack and blowing coefficient, as suggested by the experimental data. Since the expression in (5-9) is integratable, a composite expression for the empirical relations we've established for the entrainment factor is:

$$
\begin{array}{ll}
\eta_{\text {ent }}=\left(0.05 C_{j}\right)(1+0.4 \alpha) & \text { for } C_{j} \leq 0.8 \\
\left.\eta_{\text {ent }} \approx \eta_{\text {ent }}\right|_{C_{j}=0.8}+(0.0248+0.00032 \alpha)-(0.062+0.0008 \alpha) C_{j} & \text { for } 0.8<C_{j}<10
\end{array}
$$

\subsection{3 - The pressure drag factor, $\zeta$}

The pressure drag factor is simply the fraction of the pressure drag that remains unbalanced with the leading edge suction force. Unfortunately, there is so little 2-D data upon which to base this factor that only a simple empirical relationship can be implied. Consider again the pressure drag data of Figure 5-4. Note that for higher blowing 
coefficients, the rate of increase in the pressure drag is roughly equivalent for all angles of attack.

For smaller blowing coefficients, the lesser angles of attack actually experience a drag decrease with blowing. This goes back to the BLC concept. A typical USB configuration (see Figure 5-1) will have a large form drag penalty when no blowing is used. Thus, when blowing begins, it "cleans up" the flow separation and produces improved drag values. This effect is eventually over-shadowed by the high pressures induced on the aft surface and drag begins to increase.

To avoid over-complication, the drag bucket caused by BLC will not be modeled. This is because not enough information is available to fully describe it. This omission will only move the resulting approximations to the conservative side in that the drag for low blowing coefficients will be slightly over-predicted. The pressure drag factor, $\zeta$, can be modeled in several ways. One way is to create a linear function of $C_{j}$ to describe the fraction. A second way is to simply assign the factor to a constant fractional value across the range of blowing coefficients. For the former, a reasonable fit to the data is:

$$
\zeta=\left(-0.06875 C_{j}\right)+0.9875
$$

and for the latter, reasonable values could range from 0.3 to 0.5 . In other words, 30 to $50 \%$ of the aft pressure force is being realized as drag.

\section{3 - Presentation of 2-D Correlation}

The following plots show a comparison of the current USB methodology and the classical jet-flap theory with the published data described above. The current method consists of equations (4-38) through (4-40) of Chapter 4, coupled with the empirical 
relations developed in this chapter. To perform the calculations, the aft surface geometry of the airfoil shown in Figure 5-1 was created, with the surface curvature and trailing edge angle obtained from XFOIL ${ }^{(45)}$. Freestream and exit conditions were given in the published data. The experiments were assumed to take place at sea level standard atmospheric conditions. Models for the exit velocity and mass flow rate as functions of $C_{j}$ were created to match given propulsion data, so exit conditions could be simulated accurately.

\subsection{1 - Lift Coefficient}

The lift coefficient is of primary importance, since it is the crux of a USB design. Figures 5-6 through 5-10 show the sectional lift coefficient over the range $0 \leq C_{j} \leq 10$ for various angles of attack. For reference, the prediction of jet-flap theory by itself is also presented.

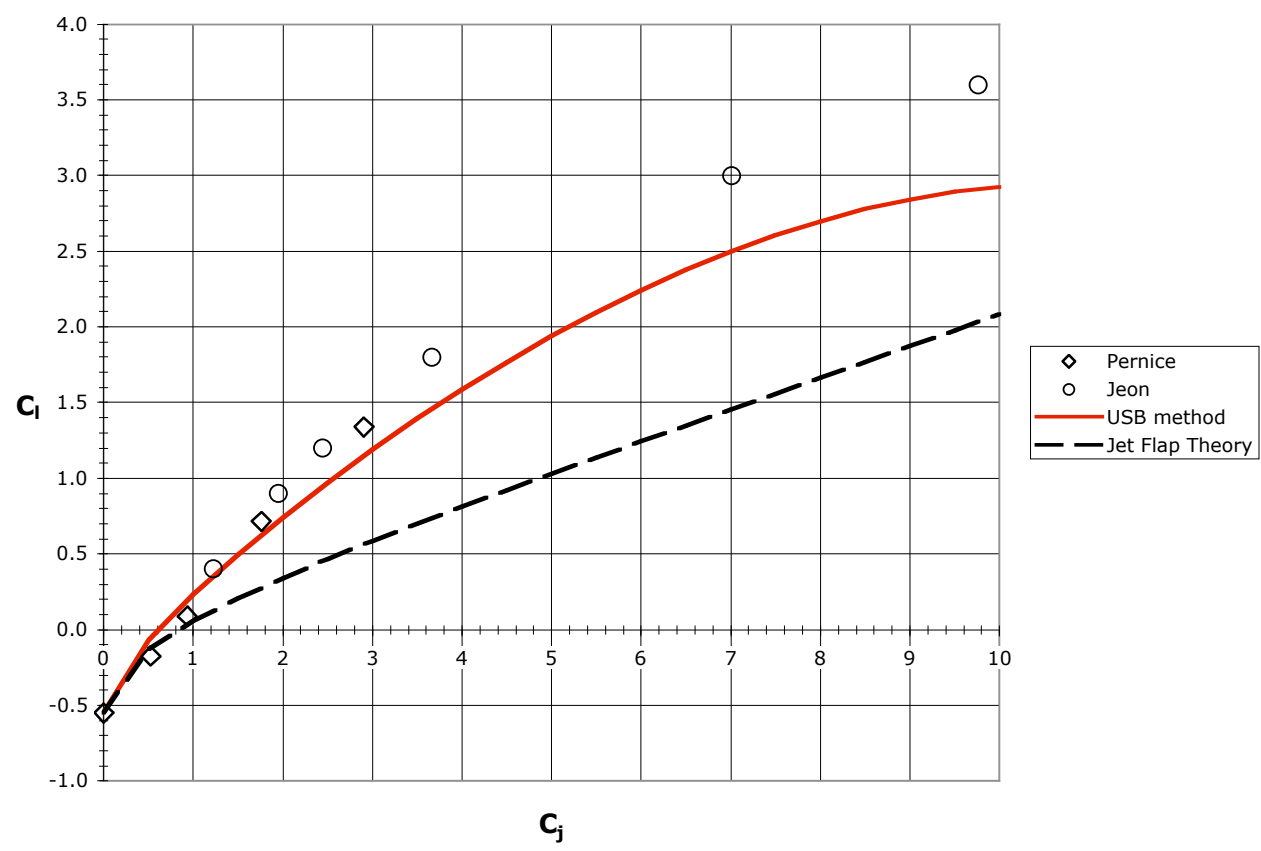

Figure 5-6: 2-D Comparison of current method, jet-flap theory, and experiment for $\alpha=-5 \mathrm{deg}$ 


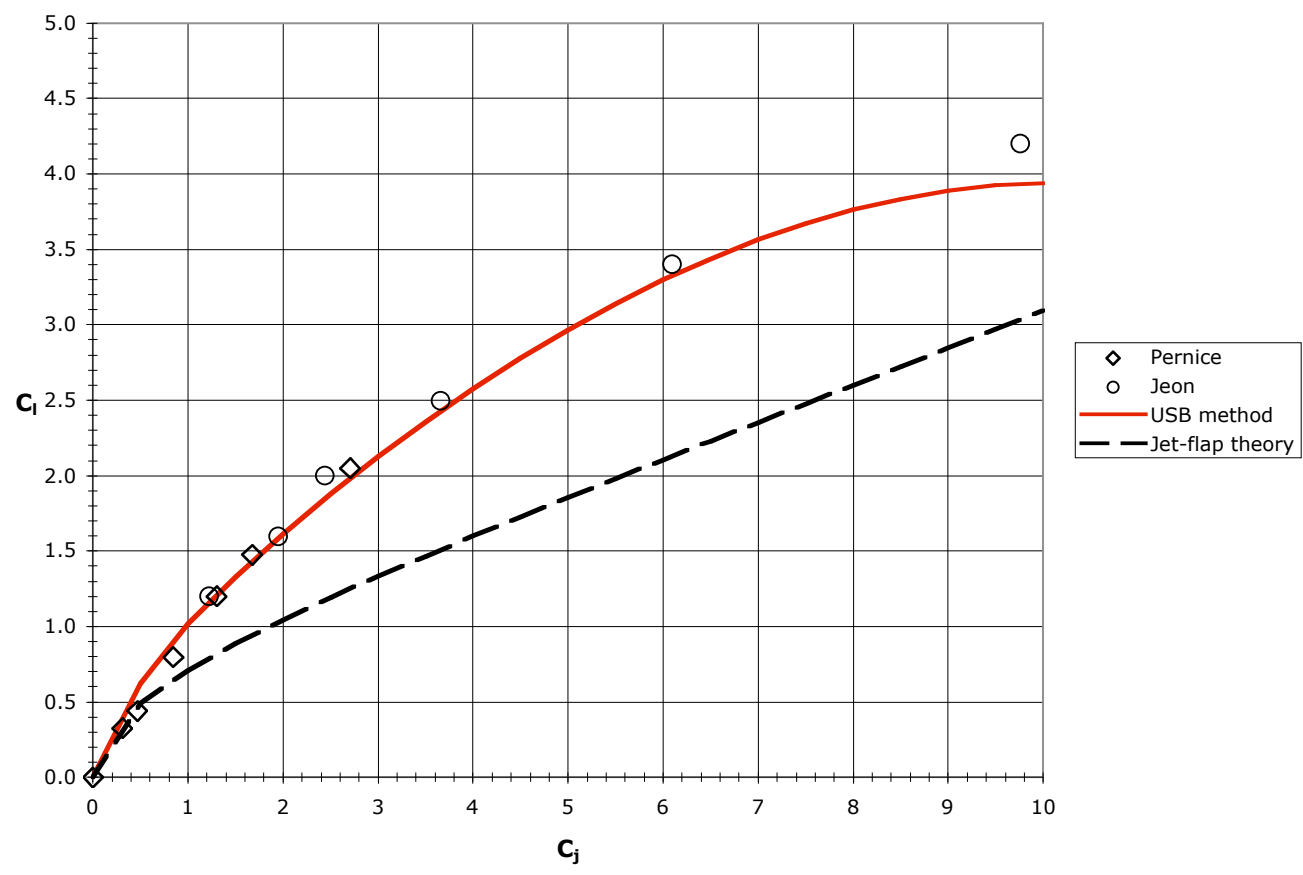

Figure 5-7: 2-D Comparison of current method, jet-flap theory, and experiment for $\alpha=0 \mathrm{deg}$

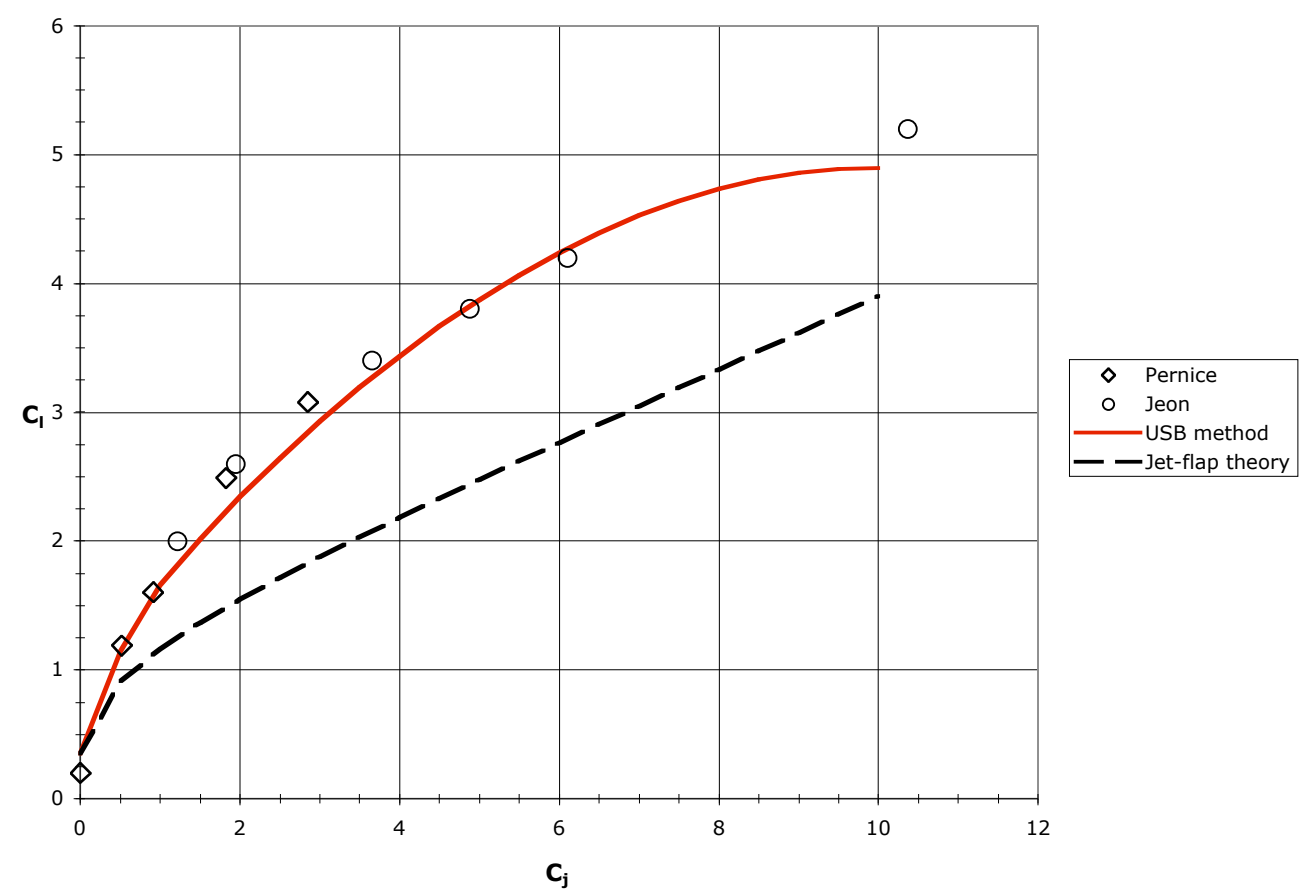

Figure 5-8: 2-D Comparison of current method, jet-flap theory, and experiment for $\alpha=5 \mathrm{deg}$ 


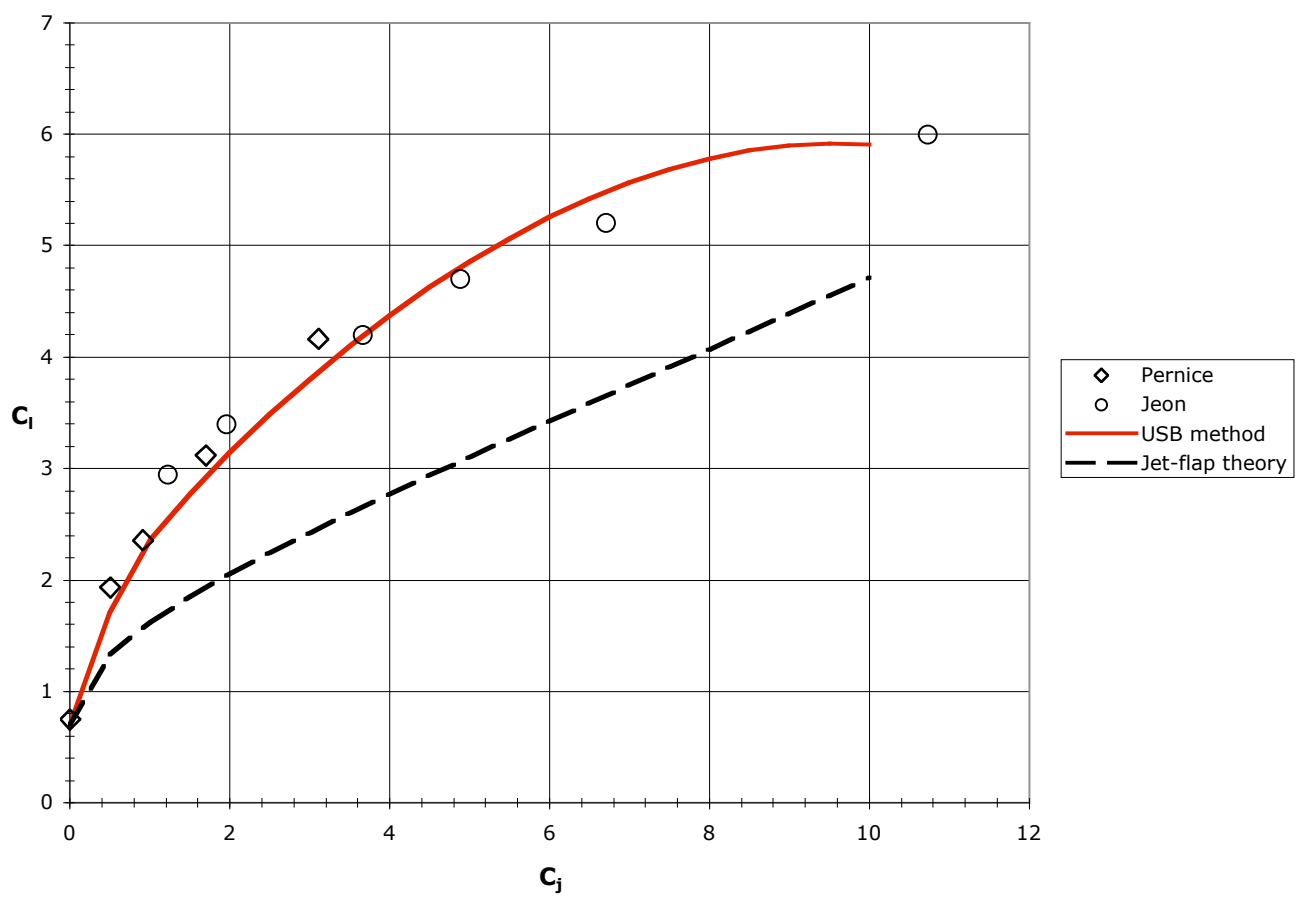

Figure 5-9: 2-D Comparison of current method, jet-flap theory, and experiment for $\alpha=10 \mathrm{deg}$

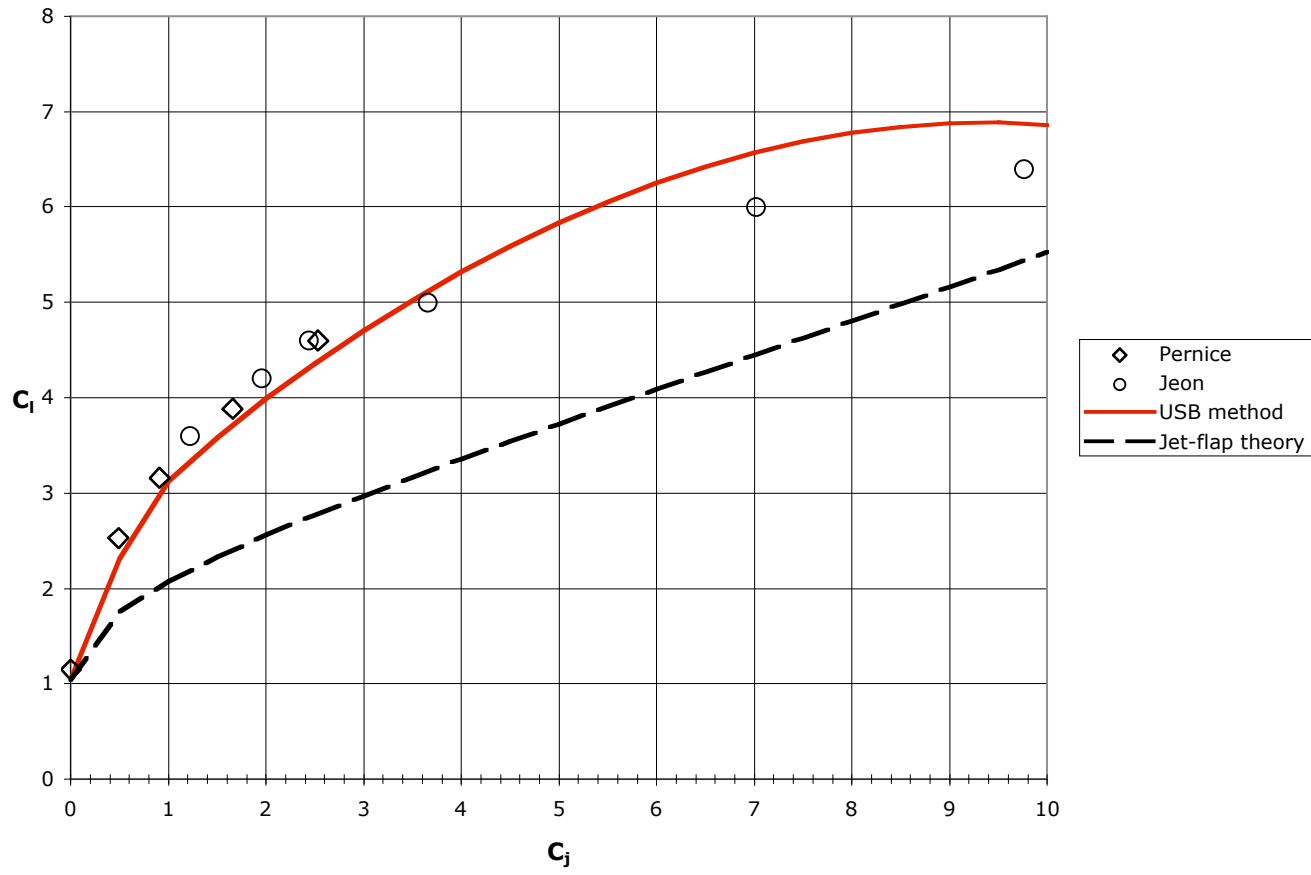

Figure 5-10: 2-D Comparison of current method, jet-flap theory, and experiment for $\alpha=15 \mathrm{deg}$ 
The results in the preceding figures show that good correlation with the experimental data can be achieved over the range of angle of attack and blowing coefficients. The inadequacy of the basic jet-flap theory is obvious throughout.

\subsection{2 - Pitching Moment Coefficient}

The pitching moment is also very important to a USB design, since these configurations can experience very large nose-down pitching moments. Since the direct reaction lift is not included, the direct reaction moment was also removed from the calculations. Figures 5-11 and 5-12 show the sectional pitching moment about the quarter-chord against the sectional lift coefficient.

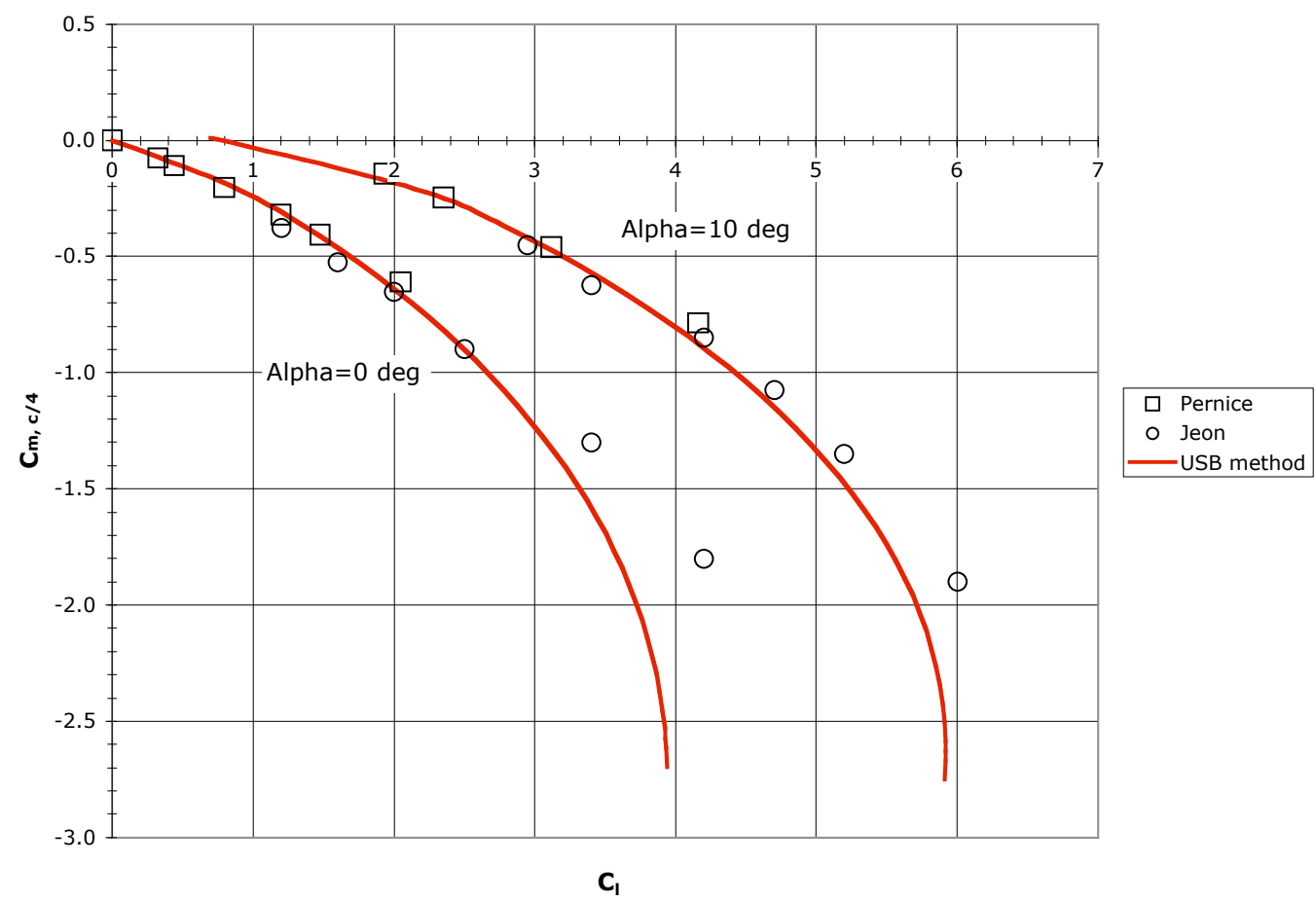

Figure 5-11: Comparison of $C_{m, c / 4}$ for current method predictions and experimental data from Ref. 43 and 44 


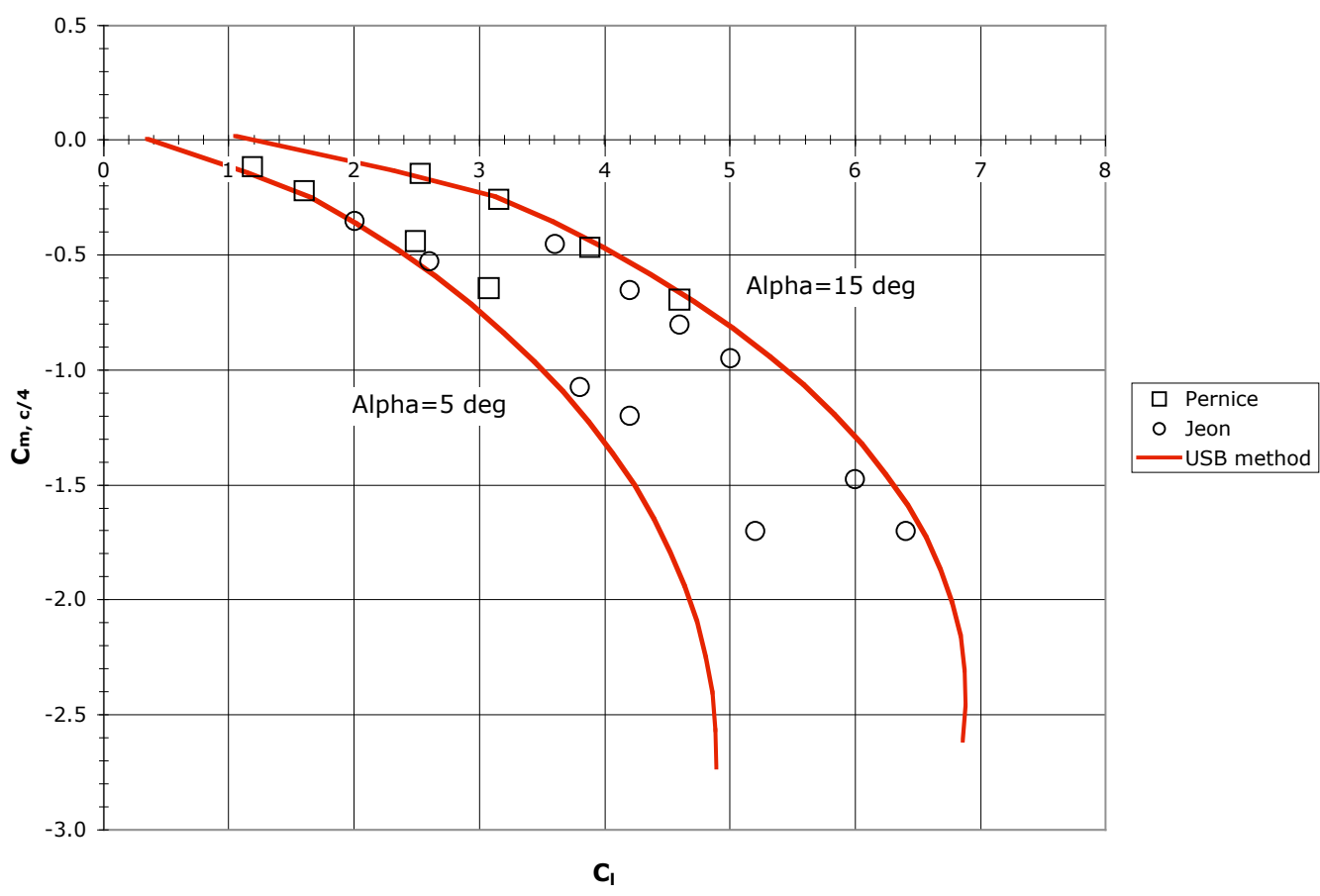

Figure 5-12: Comparison of $C_{m, c / 4}$ for current method predictions and experimental data from Ref. 43 and 44

Again, these figures show a reasonable correlation with the experimental data. It is worth noting that the current method significantly over-predicts the nose-down pitching moment for large blowing coefficients. Over the more typical, moderate range of blowing coefficients, however, the method is correlated quite well.

\subsection{3 - Drag Coefficient}

The correlation of the pressure drag is very important but is a more subjective process, as described above. The skin friction drag was ignored in the following calculations. In addition, the "zero-blowing" drag coefficient value of 0.034 was added to all values calculated. This brings the experimental and calculated drag values at $C_{j}=0$ into agreement. The pressure drag factor was modeled using equation (5-10). Figures 
5-13 through 5-16 show the sectional pressure drag coefficient with varying angle of attack and blowing coefficient.

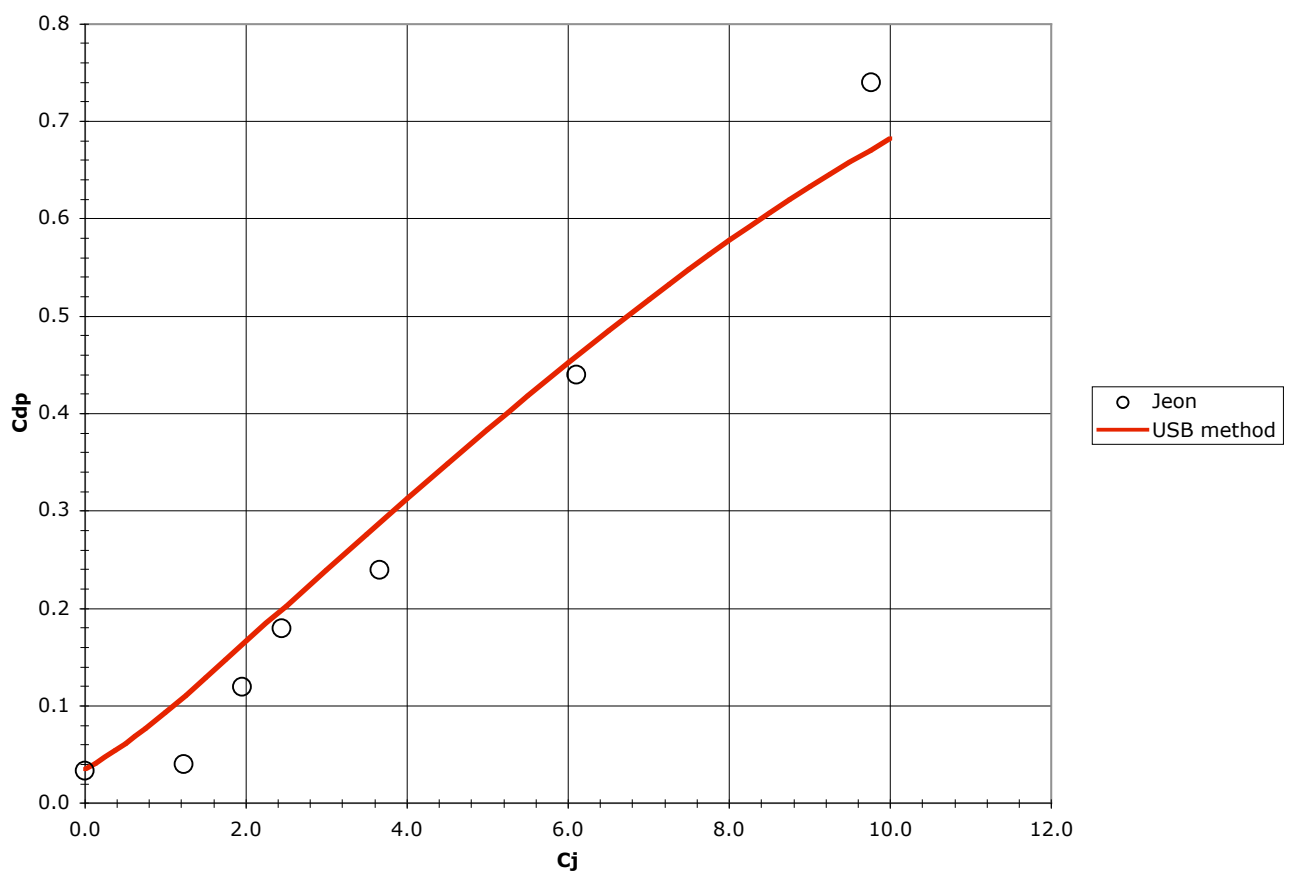

Figure 5-13: Correlation of pressure drag predictions for $\alpha=0$ deg

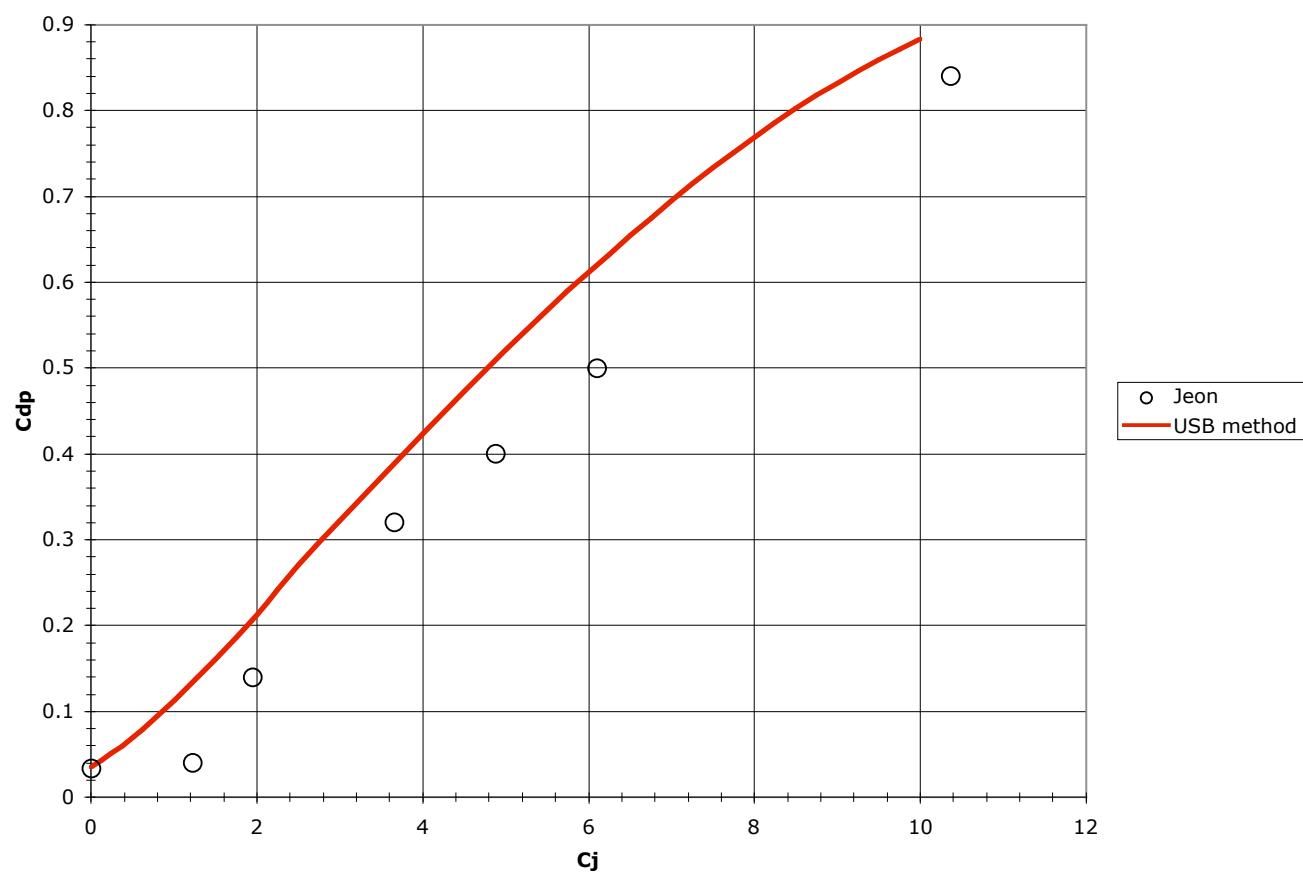

Figure 5-14: Correlation of pressure drag predictions for $\alpha=5 \mathrm{deg}$ 


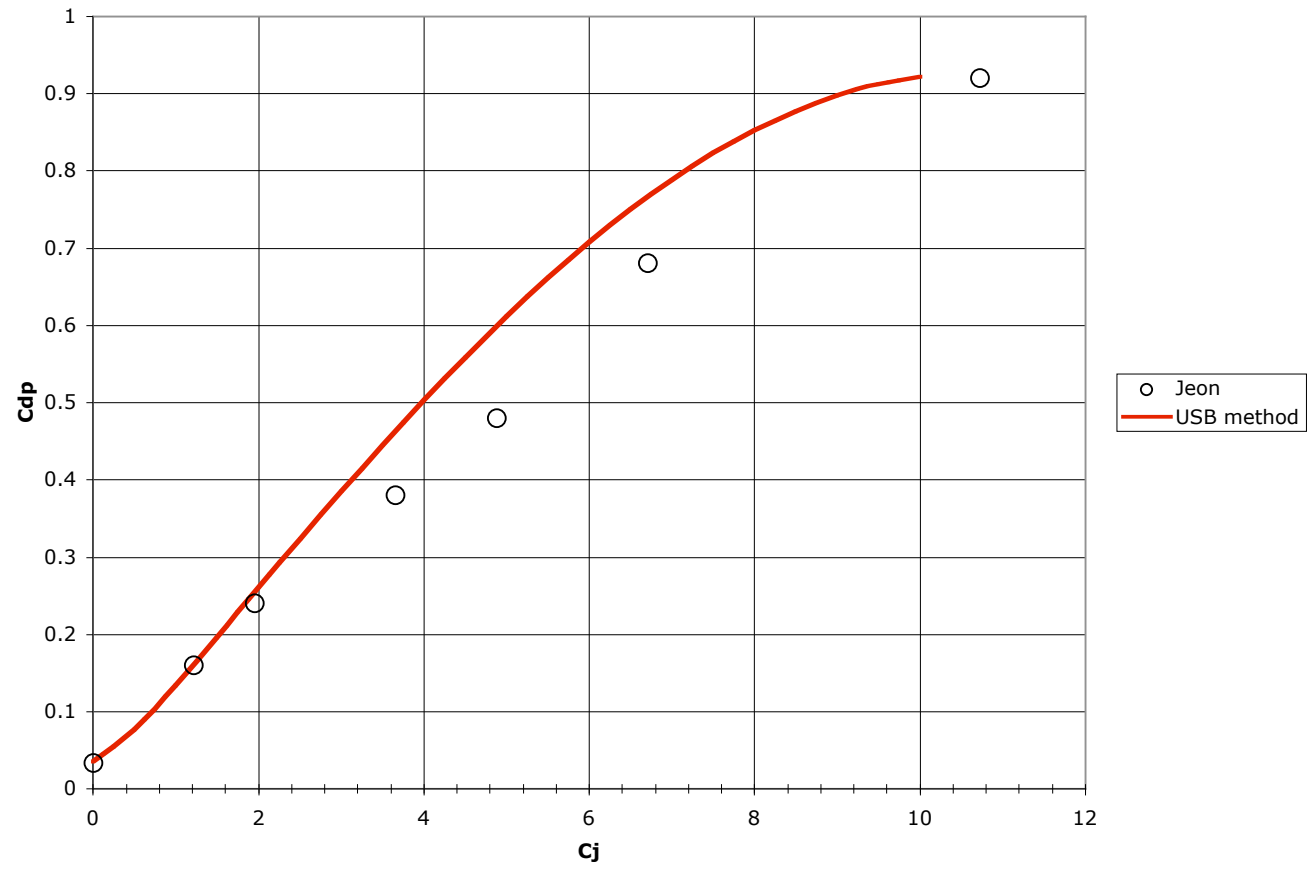

Figure 5-15: Correlation of pressure drag predictions for $\alpha=10 \mathrm{deg}$

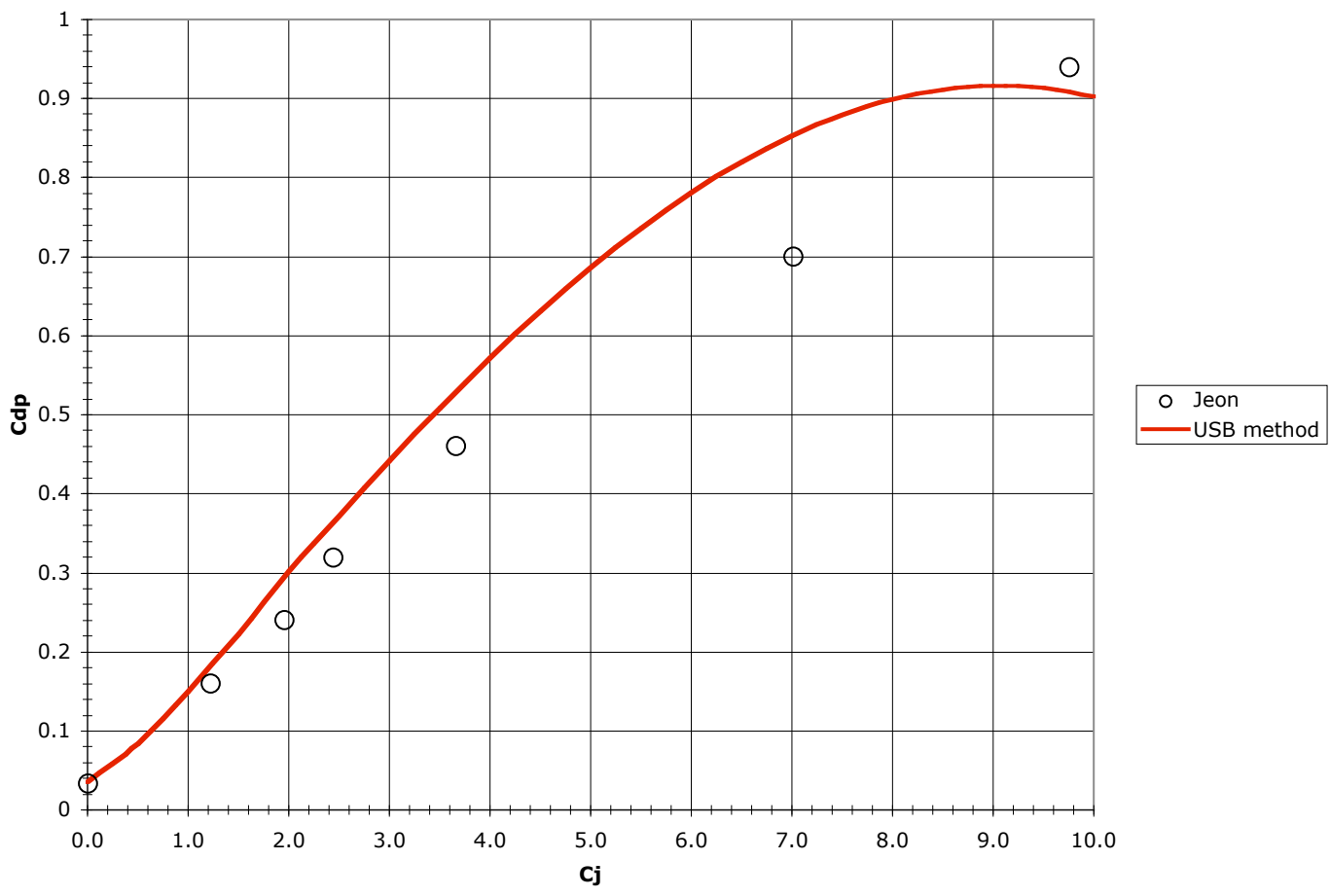

Figure 5-16: Correlation of pressure drag predictions for $\alpha=15 \mathrm{deg}$ 
While the correlation is not as good for the pressure drag coefficients, this is not unexpected. Again, since the drag is slightly over-predicted across the board, this makes the estimate conservative. 


\section{Chapter 6: Three-Dimensional USB Performance Prediction Method}

This chapter describes the method for estimating 3-D aerodynamic characteristics using the 2-D approximate method developed in the previous chapters. The estimated 2-D values are used directly in an implementation of the Weissinger lifting line method $^{(46)}$. The fundamentals of this method will be discussed along with its limitations. In addition, the process of going from 2-D to 3-D will be discussed for a general USB configuration. Finally, the additional potential of this method to provide information about engine-out conditions will be examined.

\section{1 - Overview of Weissinger Method}

For the basic calculation of the aerodynamic performance of a wing at low speed, two primary approaches are available. This first is "lifting line" theory and the second is the "vortex lattice" method. The Weissinger method can be thought of as a cross between these two in that it uses a distribution on bound vortices along the quarter-chord of the wing, but it segments the wing into chordwise "strips" or panels. Flow tangency is then 
imposed at specific control points along the wing. This method allows the coupling of sectional airfoil data with inviscid finite-wing theory. It is valid for wings of arbitrary shape, including sweep, and can handle multiple lifting surfaces. The code used for this work is a pre-existing FORTRAN implementation of a modified Weissinger method (referred to in this work as the "High-Lift Module"), that was developed by a series of researchers at the University of California at Davis. The methodology is based on theory originally developed by Weissinger ${ }^{(46)}$, and later extended by Campbell ${ }^{(47)}$ and Blackwell ${ }^{(48)}$. A more complete description of this code and the validation work done on it can be found in work by Paris ${ }^{(49)}$, Vander Kam ${ }^{(50)}$, and Roberts ${ }^{(51)}$.

The high-lift module computes the load distribution in subsonic, compressible flow for arbitrary lifting surfaces. The lifting surface is described by a system of rectangular horseshoe vortices placed along the quarter chord line. For typical symmetric situations, only half of the configuration is explicitly modeled, but asymmetric configurations require the full planform. Figure 6-1 demonstrates a planform view of the latter case.

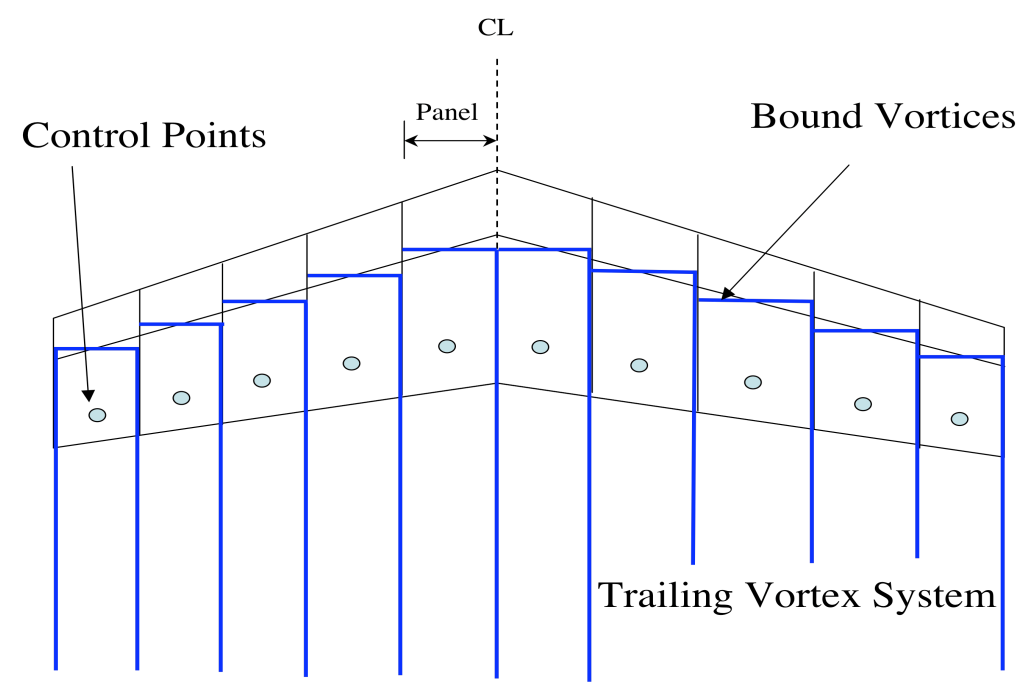

Figure 6-1: Vortex system representation of a lifting surface 
The load distribution is calculated by solving for the $N$ unknown vortex strengths while imposing flow tangency conditions at the $N$ control points. This is very similar to a vortex lattice method, but here there is only one chordwise panel.

Although the high-lift module reduces the lifting surfaces to "strips" of flat plates, various airfoil sections can be modeled. This includes any basic single-element airfoil, high-lift airfoils, and in the present case, blown airfoils. The modeling of different airfoils is accomplished by controlling the location of the control point and the effective incidence of the panel. The location of the control point can be shifted from the standard $0.75 \mathrm{c}$ to reflect the appropriate lift-curve slope, and the panel incidence can be shifted to reflect the zero-lift angle of attack of the section. Thus, portions of the wing with flaps will be treated differently than an unflapped portion based on the difference in the corresponding 2-D polars. The high-lift module also has the capability of taking viscous 2-D polars and predicting wing stall. This feature was not utilized in this work for reasons that will be described in later sections.

Once the $N$-dimensional linear system has been solved, the total aerodynamic coefficients can be calculated. The lift coefficient, $C_{L}$, is integrated directly from the calculated load distribution. The drag coefficient, $C_{D}$, is composed of the induced drag and parasite drag. The former is calculated using a Trefftz plane approach. The parasite drag is essentially the drag value specified by the sectional data and can be integrated along the span to get a total coefficient. The pitching moment coefficient, $C_{M}$, is calculated about a specified reference line (generally taken to be the aircraft center of gravity) by integrating the sectional pitching moments across the span. The sectional moments are transferred to the reference line by the following equation from Ref 49: 


$$
C_{m, x_{r e f}^{\prime}}=C_{m, x_{r e f}}+\left(C_{l} \cos \alpha+C_{d} \sin \alpha\right)\left(\frac{x_{r e f}^{\prime}}{c}-\frac{x_{r e f}}{c}\right)
$$

where $x_{\text {ref }}$ is the local quarter-chord location, and the primed value is the C.G. line.

\section{2 - Using the new 2-D USB methodology with the Weissinger Method}

Since the interest here is in having the capability to perform parametric design studies, it is important to outline how to go from a general description of the aircraft and its flight state to the aerodynamic characteristics predictions for the wing. A more detailed description is offered in Appendix B. A long-term goal of this research is to have this process entirely automated and built into conceptual design software. This would allow key variables for USB (such as nozzle geometry, flap size, or blowing span) to be used as design variables in optimization routines. The key to V/STOL or ESTOL design is the accurate incorporation of powered-lift characteristics in the earliest stages of design.

\subsection{1 - The 2-D USB estimations}

These calculations were described in Chapters 4 and 5. Starting with a given wing geometry and propulsion system layout, one must then add a flight state, meaning a specified airspeed, altitude, and flap setting. Typically the flight state will be one where USB operation is crucial, such as takeoff, landing, or "engine-out" conditions. One may also be interested in flight states such as cruise or maneuver points. Appendix B offers a complete example of generating the necessary data for the 2-D equations.

From the conditions given above, nearly all the necessary information is provided to carry out the 2-D calculations. However, a crucial distinction in the way the momentum (or blowing) coefficient, $C_{\mu}$ (or $C_{j}$ ) is determined must be made. Recall that the 
momentum coefficient was defined as the momentum flux of the jet leaving the trailing edge per unit span. In a pure 2-D sense of an infinite-span, blown wing the previous definition is again:

$$
C_{j}=\frac{\dot{m} V_{\text {exit }}}{q_{\infty} c} \approx \frac{\text { Gross Thrust }}{q_{\infty} c}
$$

However, the momentum flux per unit span in realistic cases must be tied to the actual span of the wing that is wetted by the jet exhaust. In other words, the 2-D blowing coefficient is dependent on the spanwise distribution of the jet thrust (momentum flux). The greater the span of blowing, the smaller the momentum flux per unit span. Ignoring the inevitable non-uniform distribution caused by spreading a round axial jet into a high aspect ratio sheet, the following revised definition was used:

$$
C_{j}=\frac{\dot{m} V_{e x i t}}{q_{\infty} c b_{U S B}}
$$

where $b_{U S B}$ is the span of the wing that experiences a momentum flux leaving the trailing edge. Clearly,

$$
\lim _{b_{U S B} \rightarrow b} C_{j}=\text { Gross Thrust Coefficient }
$$

for a rectangular wing. This blown span can be specified directly or used in conjunction with a nozzle design process. For example, a given exhaust spread angle may be specified and the resulting blown span calculated.

In discussing the blown span of a wing, it is important to mention another flow feature that is seldom discussed in literature, but clearly will affect results. The phenomenon of entrainment in a two-dimensional sense (and empirical approximations of these effects) was discussed in Chapter 5. However, entrainment effects naturally extend to three-dimensions. Portions of the wing adjacent to the exhaust jet will experience 
accelerated flow. The spreading angle of the jet is also increased with increasing blowing coefficient. This effect was described in the experimental work by Smith and Phelps ${ }^{(54)}$, who reported that at high blowing coefficients, the blown span was increased by over $10 \%$ and resulted in lift increments of up 6\% for the highest flap deflections. This effect is not well documented and is modeled in the current work only through providing small increases in $b_{U S B}$ for high blowing coefficients.

Once this input data has been determined, the calculations of Chapter 4 are performed to produce 2-D lift, drag, and pitching moment coefficients. As part of the current work, a MATLAB program was written to do these calculations. To demonstrate, a sample of the output data from the MATLAB code is shown in Figure 6-2 on the next page for an arbitrary geometry and flight state. The first portion of the output is merely an echo of the input data, and the table at the end represents the tabular format needed for the high-lift module.

\subsection{2 - The High-Lift Module}

Begin with the description of some of the input parameters for the high-lift module. Files using namelist input are required to define the geometry of the wing and the control parameters of the code. A complete description of these parameters is given in documentation accompanying the code. For the majority of cases run in this work, the number of vortices used along the semispan was selected to be 61 , as suggested by documentation accompanying the high-lift module. This number was large enough to have good convergence but was small enough for the code to run quickly. It is also very important to select the "non-planar wake" solution method since for powered-lift we clearly have a non-planar wake. 


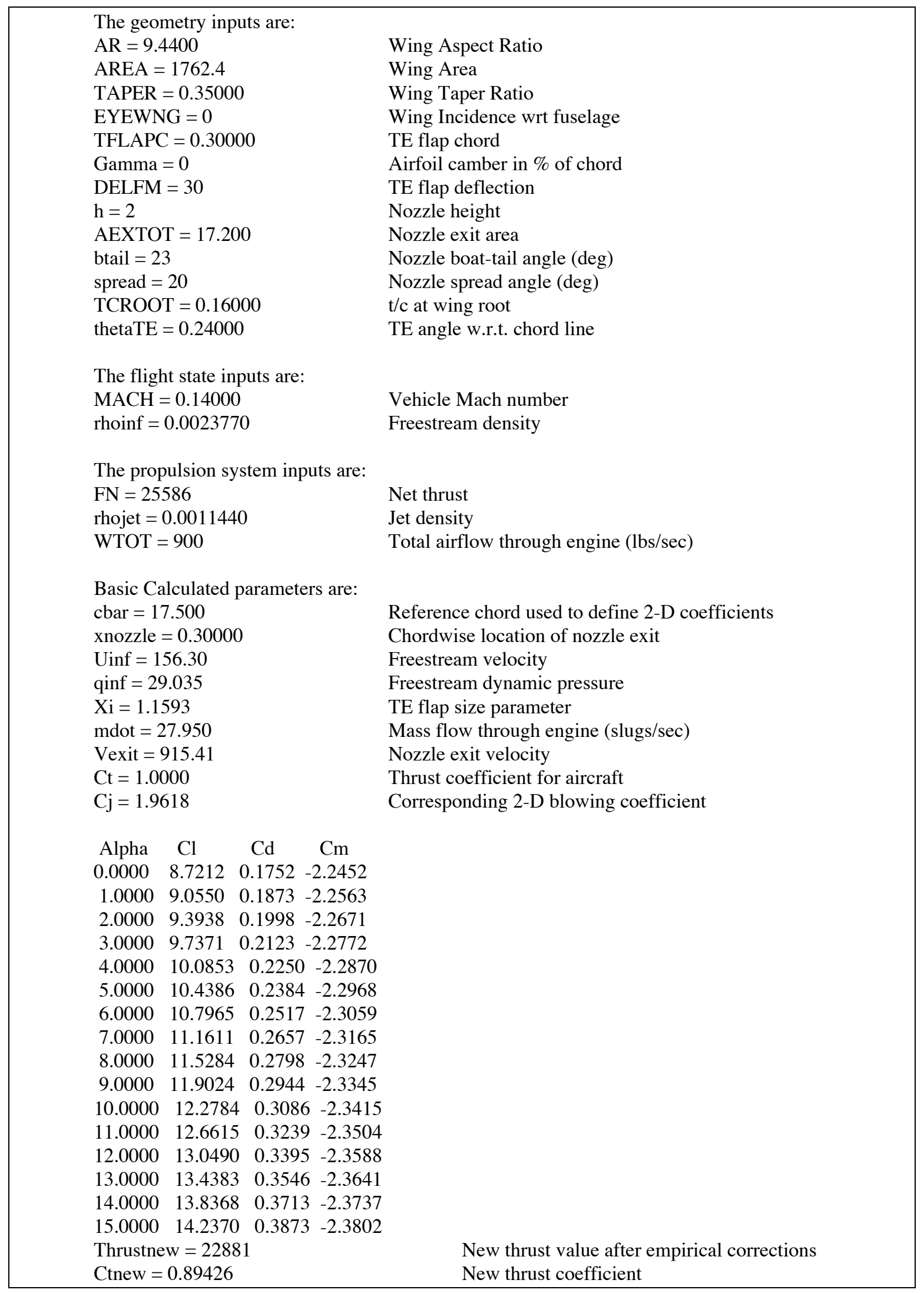

Figure 6-2: Sample output data from MATLAB 2-D USB code 
A separate file allows the user to specify the airfoil sectional data for different portions of the span. For any given spanwise location, the relevant polar is taken to be a linear interpolation between the 2-D data defined at stations bracketing the point. So, entering duplicate data at consecutive spanwise points means that all stations between those points will use identical polar data. As described above, the polars for USB sections are provided by the MATLAB code and are used directly in the high-lift module.

Non-blown sectional data was taken from various sources. Unfortunately, sectional polars for multi-element airfoil sections are not readily available. To model this type of data, lift increments were calculated using empirical methods like those from Raymer ${ }^{(52)}$. Although this introduces some error into the calculations, the magnitude of the error is small due to the relative magnitude of the polars between the blown and un-blown sections. Basic unblown airfoil data was generally obtained from Abbott and Von Doenhoff $^{(53)}$.

\section{3 - Limitations of Method}

The limitations of the 2-D USB calculations have been discussed previously. These limitations are primarily due to the breakdown of empirical approximations within the method. They may also correspond to the inability to completely model the interactions between the jet and the external flow. Thus, we can expect any approximation to deteriorate with very large blowing coefficients and/or very large flap deflections. Another fundamental consideration is that the methodology described in this work is for inviscid flow. Although viscous corrections are being made, the calculations will never 
predict flow separation of the blown sections. This is not a serious flaw for these sections however, as the jet flow will indeed prevent separation up to very high angles of attack.

There are also limitations imposed by the High-Lift module. This program was originally developed to handle modern, non-blown high lift systems. For example the complex multi-element airfoils used in large commercial transports. However, the lift coefficients developed by these sections are still considerably smaller than those dealt with in powered-lift designs. Thus, the methodology and its programming are often pushed to the limits of their present capabilities. It is, however, beyond the scope of the current work to address these limitations. Checks were made of a broad range of output to ensure that output was physically realistic, and if problems were found, to ensure that they were accounted for.

\subsection{1 - Spanload Oscillation}

One main source of discrepancies that appeared relates to the numerics involved in the very rapid change in the wing loading between blown and un-blown sections.

Typically the inboard section will be operating at much higher loadings that the outboard section. The deflected wake portion was chosen to coincide with the portion of the wing being blown. In this situation, there is a sharp change in the sectional data between the inboard and outboard sections. Figure 6-3 shows a relevant case taken from experimental work done by Sleeman and Hohlweg ${ }^{(6)}$. Note that $\eta$ is defined as the y-location/semispan. 


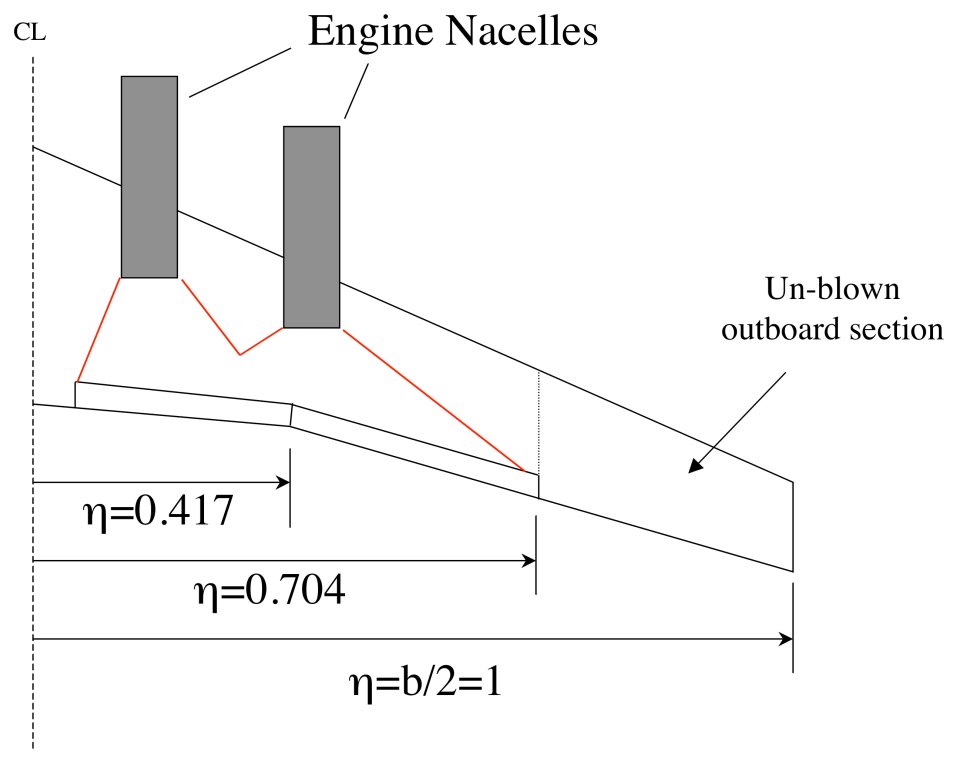

Figure 6-3: Planform view of wing used in experiments of Ref 6

In this case, the blown section/non-planar wake section was set from the wing root to $\eta=0.704$, and the outboard section was a basic NACA 6-series airfoil. Thus, there was a significant change in the relevant 2-D polar information between strips inboard of 0.704 and those outboard of that station. Figure 6-4 shows the effective angle of attack across the span for an aircraft angle of attack of $20 \mathrm{deg}$.

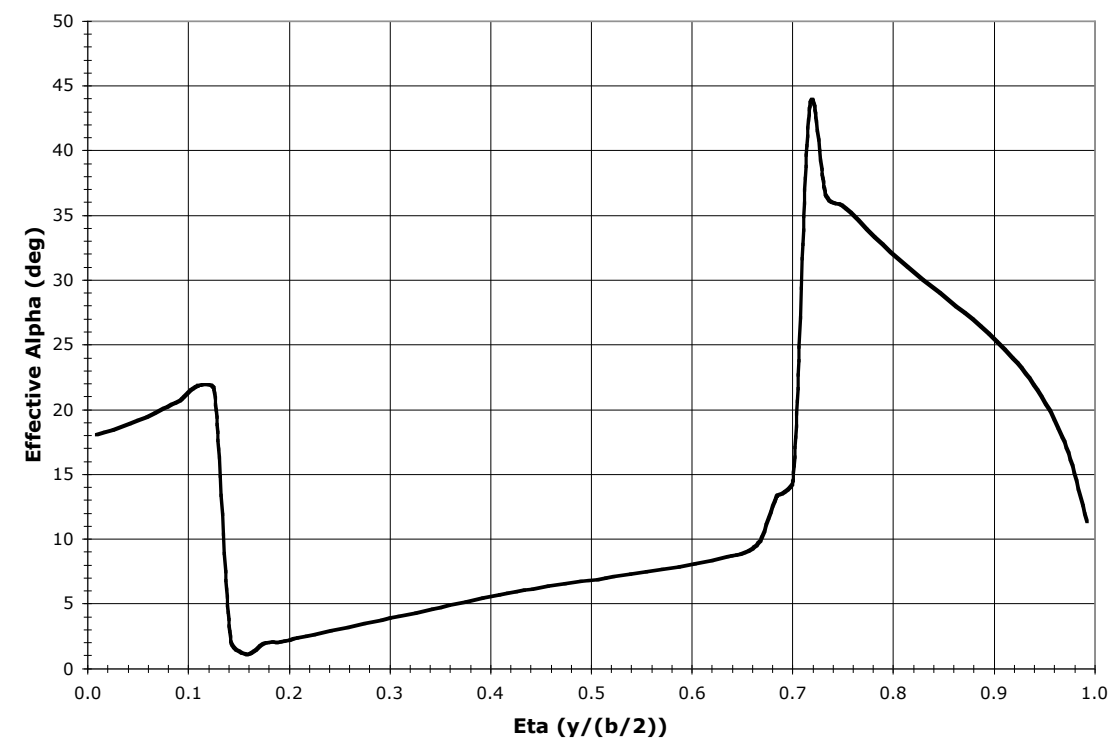

Figure 6-4: Effective angle of attack across wing semi-span 
As Figure 6-4 demonstrates, there are large jumps in effective angle of attack between the blown and un-blown sections. This is particularly true when we go from a non-planar wake to a planar wake area, where we see a change of about 35 deg within about $5 \%$ of the span. The resulting loading (given here as the local lift coefficient times the local chord) is shown in Figure 6-5.

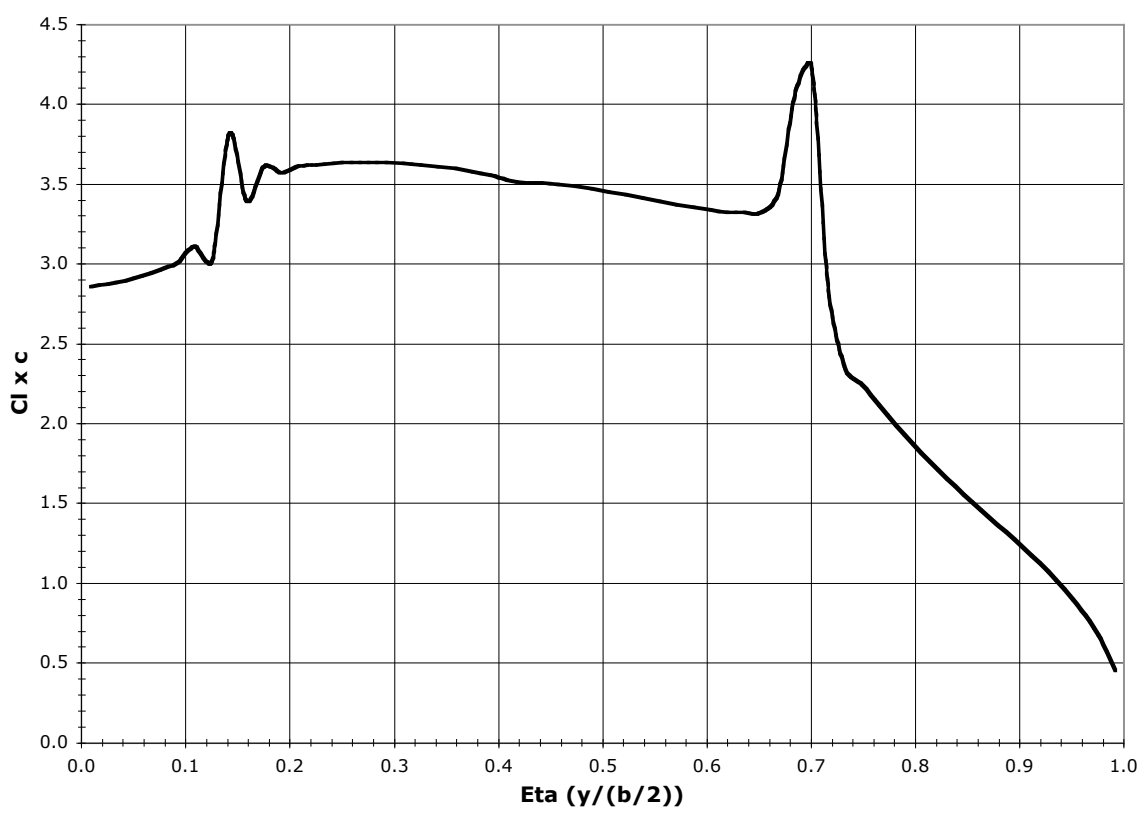

Figure 6-5: Wing loading for the case shown in Figure 6-4

The oscillations in regions of sharp variation of effective alpha are clear. While the inboard oscillations aren't good, it's the outboard spike that is more important due to its relative magnitude. In fact, this problem becomes more severe for cases with higher wing loadings. For example, in a twin-engine configuration, the oscillations are greater in magnitude. As described in Chapter 2, a vortex is present at the edge of the jet sheet. We certainly do expect a sharp decrease in the wing loading at this point, but the oscillating behavior is not physically realistic.

The use of a manually imposed "buffer region" was investigated where the 2-D polars were allowed to decrease linearly between the sections. While this did reduce the 
magnitude of these oscillations, it did not eliminate them. We hypothesize that the reason for the oscillations is because the method is being forced to extrapolate the data well beyond it's input range. This fact, along with going from a non-planar wake to a planar wake region, is responsible for the large spike experienced around $\eta=0.70$.

The significance of these oscillations lies primarily in the determination of induced $\operatorname{drag}, C_{D i}$. While lift is also affected, the oscillations tend to cancel one another out leaving the lift largely unchanged. Based on a Trefftz-plane derivation by Mason ${ }^{(15)}$, the induced drag of a wing can be given by:

$$
D_{i}=\frac{-\rho_{\infty}}{4 \pi} \int_{-b / 2}^{b / 2} \int_{-b / 2}^{b / 2} \frac{d \Gamma\left(y_{1}\right)}{d y} \frac{d \Gamma\left(y_{2}\right)}{d y} \ln \left(y_{1}-y_{2}\right) d y_{1} d y_{2}
$$

We see that the induced drag is directly tied to the changes in the spanloading. Sharp breaks or oscillations would mean that the induced drag becomes large. As will be shown in the following chapter, for situations involving high wing loadings, this oscillation did indeed dramatically increase the induced drag calculated.

The most obvious method to correct the excessive induced drag predictions is to manually remove the oscillations from the spanloading. This procedure was investigated for a representative case from Smith and Phelps ${ }^{(54)}$ with $\delta_{f}=20^{\circ}$ and $\alpha=25^{\circ}$. The highlift module output for this case was $e=0.364$. Figure $6-6$ shows the original output spanload and the modified spanload with the oscillations removed: 


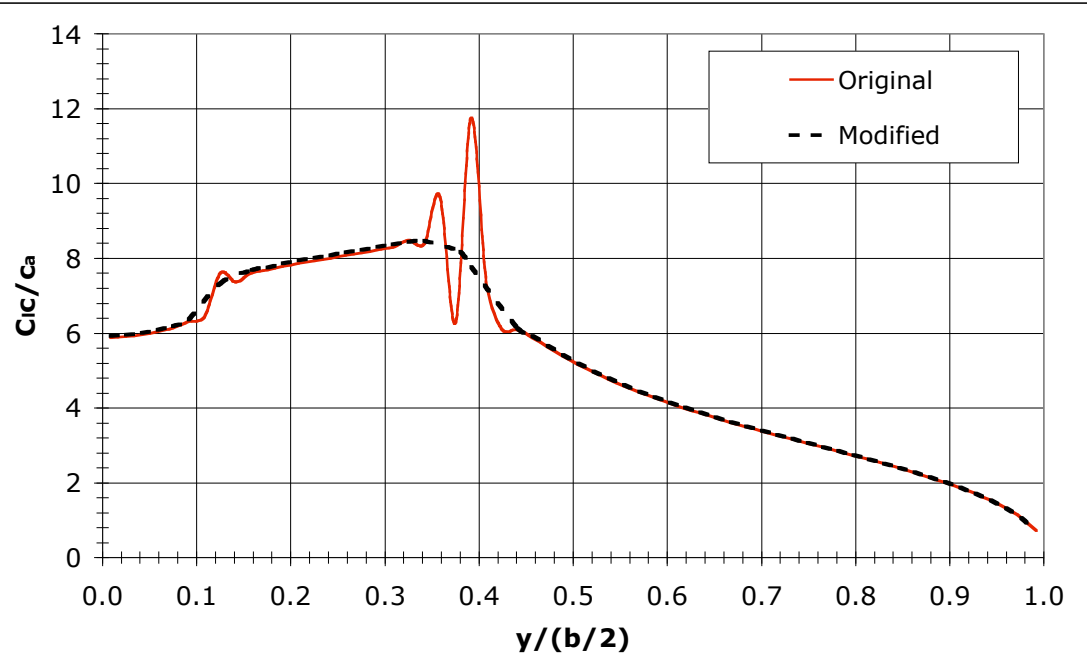

Figure 6-6: Original and corrected wing spanloadings

Using an induced drag calculation where the spanload is modeled using fourier series, the span efficiency factor was computed for each case. It was found that the original oscillating spanload produced $e=0.359$, while the modified loading produced $e=0.74$. These results agree well with the predictions of a non-linear regression code written for this work that will be discussed in the next chapter. Because manually smoothing the spanloading in each case would require excessive time and effort, the non-linear regression method to obtain $e$ (and, importantly, the vertex of the drag polar) is preferred in correcting this problem.

\subsection{2 - Stall Predictions}

As mentioned previously, the High-Lift module has the capability to predict wing stall and thus $C_{L, \max }$. Since experimental 2-D polars can be used, the loss of lift can be modeled and a peak value of the lift coefficient will be reached. In the event an airfoil is pushed beyond stall, the program can apply basic post-stall behaviors to help the solution converge. This basically consists of adding a linear portion to the post-stall region ${ }^{(51)}$. Since the blown sections do not show stall behavior until large angles of attack, the un- 
blown sections of the wing may stall without the aircraft experiencing the dramatic loss of lift of a conventional design. The "stall" event for a USB aircraft configuration is more appropriately viewed as the point at which the outboard control surfaces stall.

In the previous section it was demonstrated that wing sections adjacent to blown sections experience a large upwash and are placed at a much larger effective angle of attack. If viscous data is input, significant portions of the unblown section may be well within the post-stall region. Numerically, if the post-stall corrections are not used within the code, it will not converge to a solution. If post-stall corrections are used, the quality of the output is highly dependent on the nature of the corrections. For this reason, only unstalled 2-D polars were input, with the assumption being that sufficient leading edge protection was present to delay stall behavior.

\subsection{3 - Nacelle and Fuselage Effects}

The High-Lift module was developed to accurately predict wing performance. As such, only limited work was done on incorporating effects from other aircraft components. There are currently some routines for estimating fuselage effects. They consist of modifying the upwash field near the wing/fuselage junction. Since these modifications have not been completely validated, this option was not used in any calculations in the current work.

The engine nacelles are another component that can significantly contribute to the aerodynamics. No force or pitching moment corrections are currently in place to address the presence of the engine nacelles. The two main areas where this modeling would be useful are in the additional friction drag they incur and the variations in $C_{M, \alpha}$ they can 
produce. For cases with swept wings and long nacelles, the force produced on the duct can produce a large pitching moment. Nacelle effects were modeled by Mendenhall ${ }^{(13)}$ in his vortex lattice method using slender body theory to predict the lift force and center of pressure. A similar approach could easily be used for the current method.

\section{4 - Lateral-Directional Characteristics}

The ability to enter information about the entire aircraft wingspan can make the highlift module useful in other ways. The engine-out case will always be an active constraint for powered-lift designs. An aircraft built for very low stall speeds with high wing loading and lift coefficients may be completely uncontrollable with one engine inoperative. In previous USB designs, a significant amount of research was done to help minimize the effects of losing an engine. The most successful was the use of BLC on the dead engine side and opening the USB flaps to function like multi-element flaps. Both of these features could be modeled. Figure 6-7 shows how this could be done.

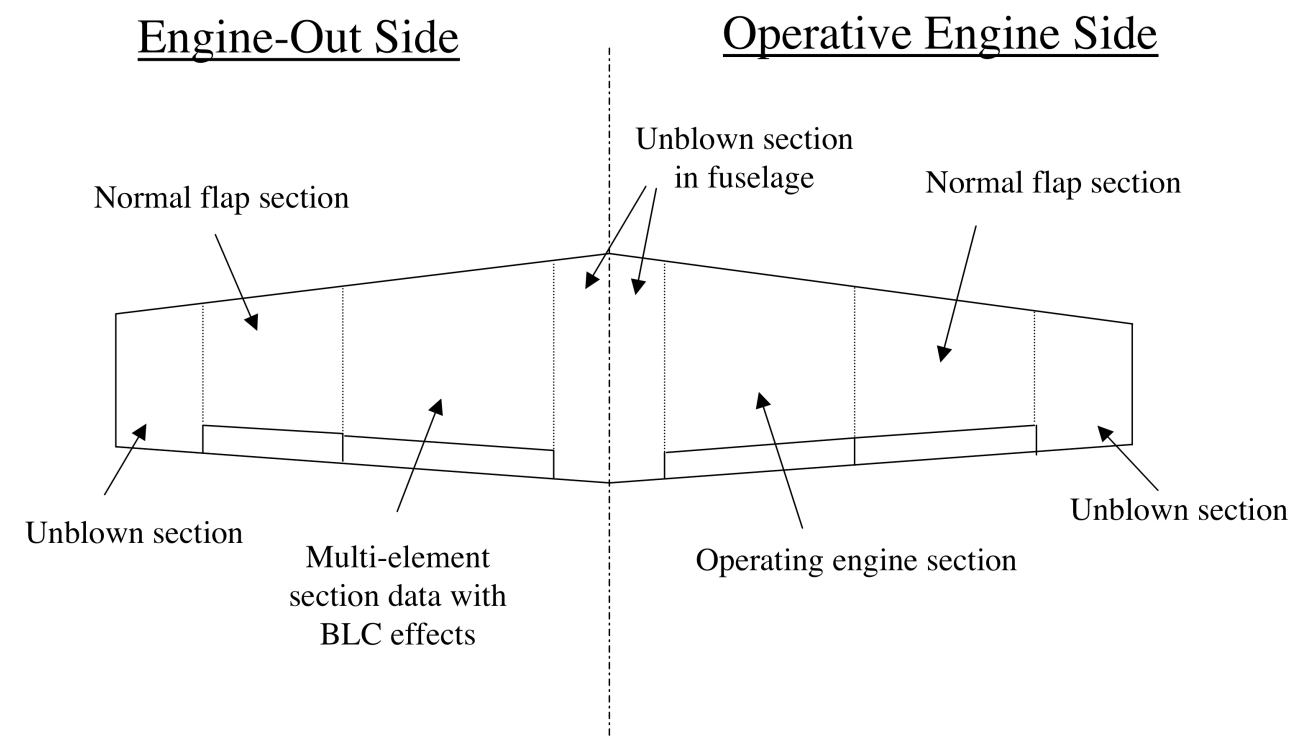

Figure 6-7: Sectional data regions across a wing for an engine-out case 
Once we know the $C_{N}, C_{l}$, and $C_{Y}$ contribution from the wing, the control authority to maintain a given heading or orientation can be found from the equations of motion. This process would work equally well for estimating the forces and moments we can generate to assist in maneuvers. It should be noted that the ability of the current method to operate in this aspect is crucial to facilitate the design decision process in the early design stages. 


\section{Chapter 7: Comparison with 3-D Experimental Results}

This chapter presents direct comparisons of the new USB methodology with experimental data. This data was obtained from a variety of sources, with the primary sources being Technical Notes published by NASA. The wind tunnel models include a wide range of scales and configurations. Because of this, individual cases had to be modeled differently to approximate the flap system and propulsion conditions appropriately. Any corrections or adjustments made to capture other influences will also be discussed. It was found that the current method typically approximated the magnitude of aerodynamic coefficients within $10 \%$ and also captured derivative information $\left(C_{L \alpha}\right.$, $C_{m \alpha}$ ) very well over a broad range of inputs.

We also present a short comparison of the new USB methodology with an engineering method produced by Mendenhall and Spangler ${ }^{(13)}$. This method is based on a non-linear vortex lattice method for USB aircraft. The authors also performed comparisons with NASA Technical Note data sets. It was found that the current methodology compares very well with the non-linear VLM. 


\section{1 - NASA Experimental USB Reports}

This series of publications ${ }^{(6),(54),(55)}$ was based on work done in the NASA Langley fullscale tunnel between 1973 and 1975. Three of the studies were selected for comparison with the current method based on the large amount of data presented and the wide range of aircraft configurations tested. These configurations include twin vs. four-engine models, varying spans of blowing, combinations of USB and slotted high-lift devices, and very different planform areas. Table 7-1 provides a comparison of the three configurations used in our comparisons:

Table 7-1: Parameters for three USB wind-tunnel models

\begin{tabular}{|c|c|c|c|}
\hline \multirow[t]{2}{*}{ Parameter } & \multicolumn{3}{|c|}{ Value used in calculations } \\
\hline & TN 8061 & TN 7526 & TN 7399 \\
\hline Aspect Ratio & 7.48 & 3.90 & 7.0 \\
\hline Wing Area & $5.669 \mathrm{ft}^{2}$ & $89.74 \mathrm{ft}^{2}$ & $4.86 \mathrm{ft}^{2}$ \\
\hline Wing Taper & 0.247 & 0.73 & 0.391 \\
\hline $\begin{array}{l}\text { TE flap chord ratio } \\
\left(c_{\mathrm{f}} / \mathrm{c}\right)\end{array}$ & 0.35 & 0.30 & 0.30 \\
\hline $\begin{array}{l}\text { Avg. chord for USB } \\
\text { section }\end{array}$ & $0.951 \mathrm{ft}$ & $5.5 \mathrm{ft}$ & $1.2 \mathrm{ft}$ \\
\hline Airfoil section type & supercritcal & NACA $64{ }_{2} \mathrm{~A} 215$ & NACA 4415 \\
\hline Airfoil TE angle & $10.3 \mathrm{deg}$ & $12 \mathrm{deg}$ & $14.3 \mathrm{deg}$ \\
\hline Nozzle exit area & $0.0495 \mathrm{ft}^{2}$ & $1.667 \mathrm{ft}^{2}$ & $0.0417 \mathrm{ft}^{2}$ \\
\hline Nozzle height & $0.091 \mathrm{ft}$ & $0.646 \mathrm{ft}$ & $0.10 \mathrm{ft}$ \\
\hline $\mathrm{b}_{\mathrm{USB}}$ & $1.8 \mathrm{ft}$ & $7.4 \mathrm{ft}$ & $1.547 \mathrm{ft}$ \\
\hline t/c@wing root & 0.093 & 0.15 & 0.15 \\
\hline $\begin{array}{l}\text { Spanwise location of } \\
\text { engine(s) }\end{array}$ & $0.255 \mathrm{~b} / 2,0.418 \mathrm{~b} / 2$ & $0.259 \mathrm{~b} / 2$ & $\begin{array}{l}0.27 \mathrm{~b} / 2,0.423 \\
\mathrm{~b} / 2\end{array}$ \\
\hline Test Mach number & 0.104 & 0.03 & 0.047 \\
\hline $\begin{array}{l}\text { Test dynamic } \\
\text { pressure }\end{array}$ & $16 \mathrm{lb} / \mathrm{ft}^{2}$ & $1.65 \mathrm{lb} / \mathrm{ft}^{2}$ & $3.31 \mathrm{lb} / \mathrm{ft}^{2}$ \\
\hline
\end{tabular}

The following sections will look at each of these cases in detail.

\subsection{1 - Case 1: Small-scale, Four-engine configuration (TM D-8061)}

This work was performed by Sleeman and Hohlweg( ${ }^{(6)}$. It was a low-speed investigation of the powered-lift aerodynamic performance of a four-engine USB transport. The model had swept wings with rectangular exhaust nozzles. More detailed 
parameters were given in Table 7-1, and Figure 7-1 shows a sketch of the aircraft wing planform:

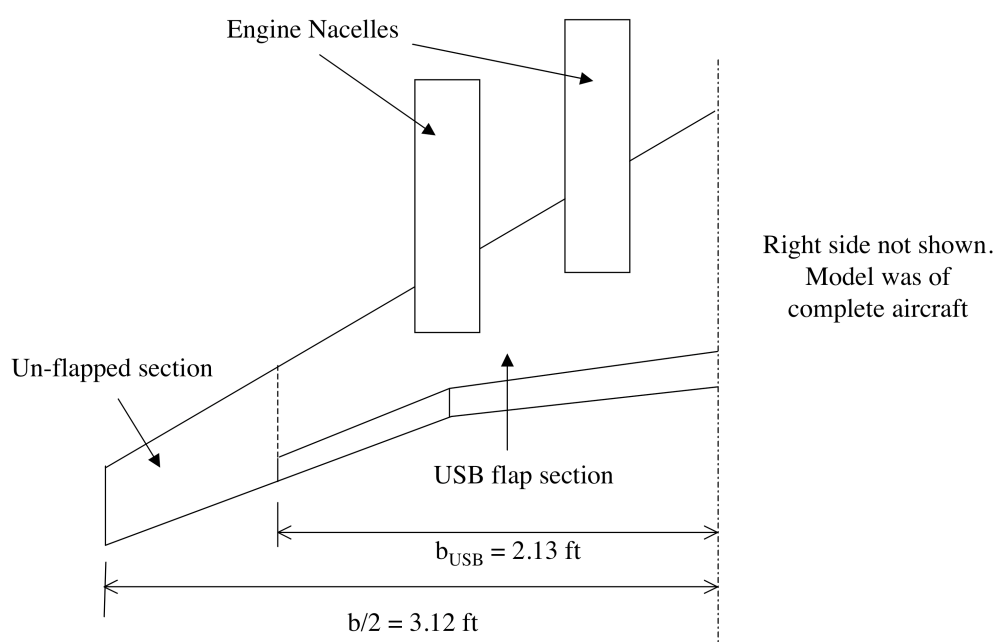

Figure 7-1: Wing model of aircraft tested in Ref. 6

The total thrust coefficient, $C_{T}$, was used as the correlating parameter in the study. It was defined as:

$$
C_{T}=\frac{\text { Total thrust }}{q S}
$$

This was used to find the thrust from each engine at a given throttle setting. This thrust was used directly to find the corresponding blowing coefficient per unit span, $C_{j}$. However, since the Circular Streamline Theory requires the a value for the jet exit velocity, this thrust value was also used to approximate the exit velocity and mass flow through the engines simulator by assuming it was operating at the design condition. As an example, consider the $C_{T}=2.0$ case. Using equation (7-1) the total thrust is $181.4 \mathrm{lbs}$, and thus each engine produces $45.35 \mathrm{lbs}$ thrust. As described in section 6.2.1, the blowing coefficient was defined as:

$$
C_{j}=\frac{\text { Engine thrust }}{q c b_{U S B}}
$$


In this case we have 2 engines per side blowing our USB flaps, which leads to a momentum flux per unit span of blowing of 3.31. This means that at $C_{T}=2.0$, the flow over portions of the wing wetted by the jet will perform similar to a 2-D airfoil operating at $C_{j}=3.31$.

Comparisons were made with experimental data at thrust coefficients of 0.9, 2.0, and 3.0 (where available) at flap settings of 0 and 35 degrees over a range of angle of attack from -5 to 25 degrees. Figures $7-2$ through $7-5$ show the results of the comparison at 0 deg angle of attack.

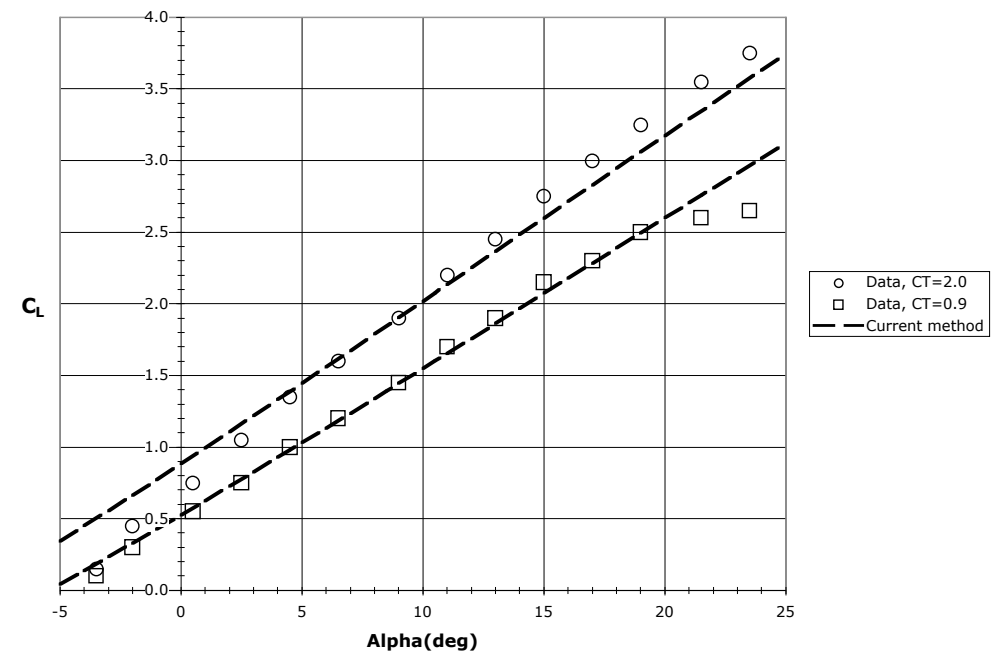

Figure 7-2: $C_{L}$ vs. Alpha for 0 deg flaps at 2 thrust settings

The calculations compare favorably with the experimental data, although we cannot predict the stall that is evident at around $\alpha=20 \mathrm{deg}$. It is also interesting to note in Figure 7-2 that for 0 deg flaps, it is easy to see how the increasing thrust coefficient produces a change in lift curve slope. This effect is present for any flap deflection, since it can be shown from equation (4-38) that $C_{L \alpha}$ is function of $C_{j}$. However, in this case the small changes in reaction lift and pressure lift with increasing $C_{j}$ show the slope increase more clearly (i.e. the zero-lift angle of attack, $\alpha_{O L}$, varies only slightly). This effect is 
present in the current method, but fails in this case to fully capture the $C_{L \alpha}$ increase. At a higher flap deflection, this effect will be harder to directly see since increases in blowing coefficient will produce large upward shifts in the lift curves (large changes in $\alpha_{O L}$ ).

Figure 7-3 shows a comparison of the aircraft pitching moment:

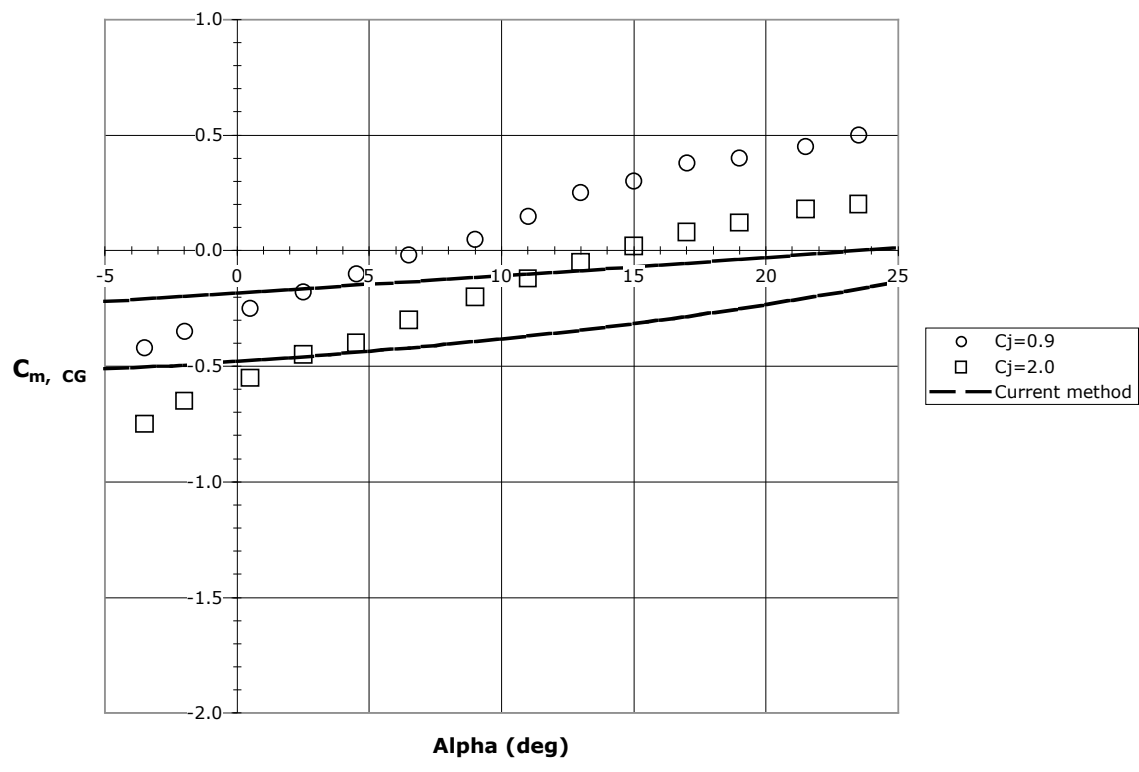

Figure 7-3: $C_{m}$ vs. Alpha for 0 deg flaps at 2 thrust settings

Figure 7-3 shows that the general magnitudes of the pitching moments agree, though the slope is clearly incorrect. We believe that this error in $C_{m \alpha}$ is due to the absence of calculations to account for the effects of the engine nacelles or ram drag. As the aircraft changes angle of attack, forces are generated on the nacelle and ram drag may become large. These forces are acting significantly forward of the C.G. location for a swept wing with long nacelles, making the "nose-up" characteristic more pronounced. Figures 7-4 and 7-5 show the aircraft "thrust-plus-drag" polars: 


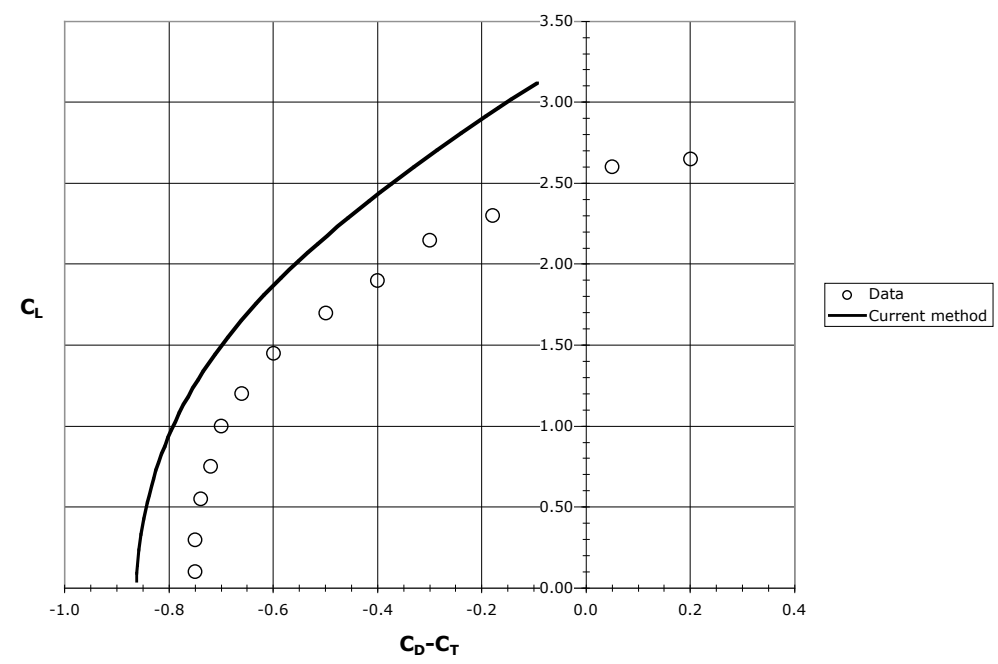

Figure 7-4: Thrust-plus-Drag polar for $C_{T}=0.9,0$ deg flaps

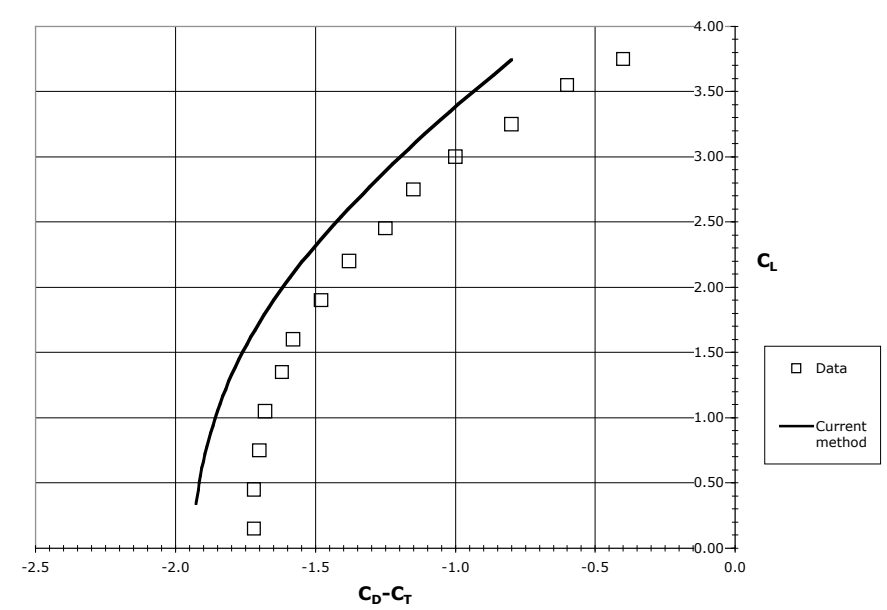

Figure 7-5: Thrust-plus-Drag polar for $C_{T}=2.0,0$ deg flaps

The thrust-plus-drag polars compare very favorably to the experimental data. While the magnitude is predicted to only about $15 \%$, the curve trends are predicted very well. This criteria is more important in a low-fidelity methods where the absolute magnitude is less critical than capturing how quantities change with variations in design variables.

Similar results were obtained when the USB flaps were deflected to $35 \mathrm{deg}$. In the analytical model, the flaps were modeled as a constant radius curve that incorporated the appropriate amount of chord extension for this flap system. The lift curve is presented below: 


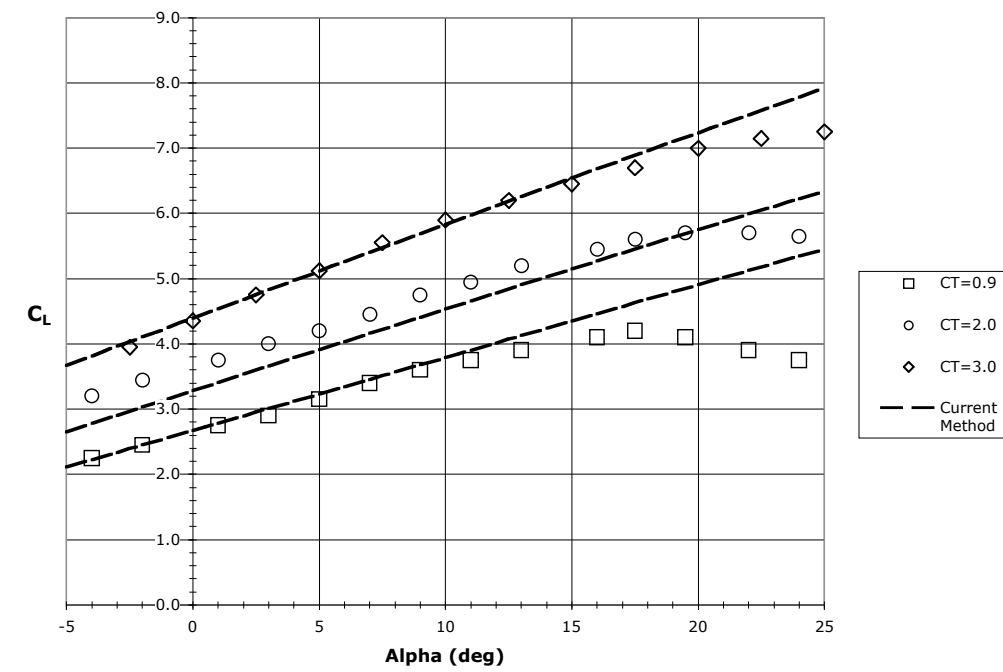

Figure 7-6: $C_{L}$ variation with Alpha for 35 deg USB flaps at 3 thrust settings

Note that with the flaps deflected the increase in thrust coefficient (and thus blowing coefficient) leads to more of a shift in the lift curve than an increase in $C_{L \alpha}$. The current method matches the data well. Figure 7-7 shows the pitching moment curves:

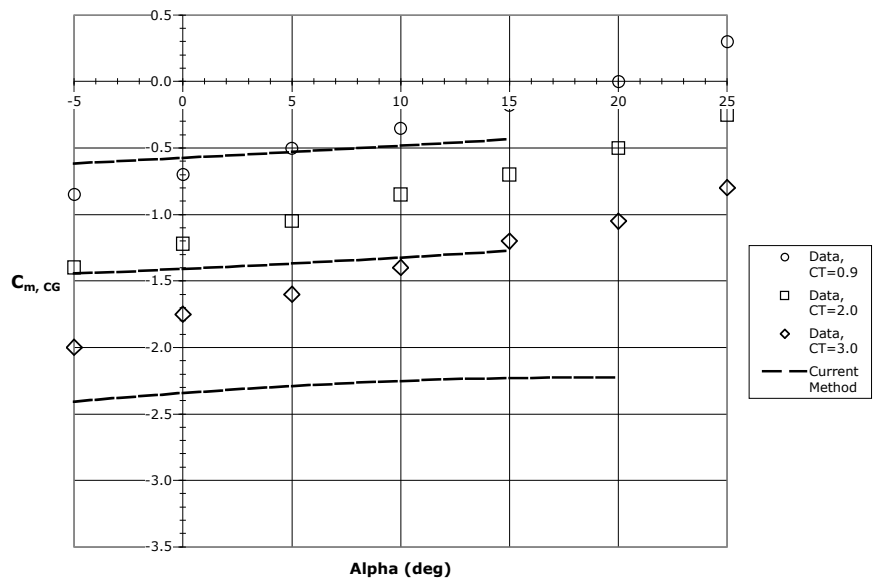

Figure 7-7: Pitching moment about the C.G. vs. Alpha for 35 deg USB flaps at 3 thrust settings

The thrust-plus-drag polars are shown in Figure 7-8. Note that the slopes are matched reasonably well while the magnitude starts to agree less with increasing thrust coefficient. Some discrepancy in the polar shape (the " $E$ " value) is expected due to the additional induced drag caused by the spanload oscillations. 


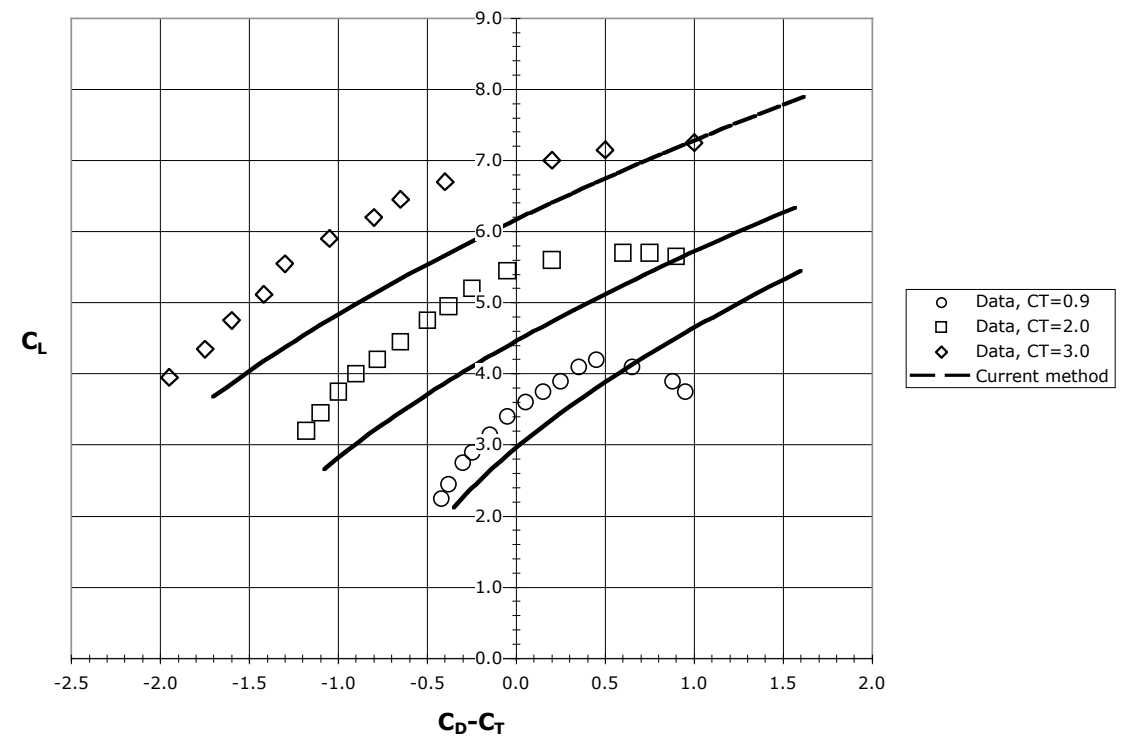

Figure 7-8: Thrust-plus-Drag polars for 35 deg flaps at 3 thrust settings

\subsection{2 - Case 2: Large-scale, Twin-engine configuration (TN D-7526)}

The second technical note considered was published by Smith and Phelps ${ }^{(54)}$. These experiments investigated the longitudinal aerodynamic characteristics of a large-scale semispan model with an unswept wing. The semispan model had 1 engine modeling a twin-engine aircraft configuration as opposed to the four-engine model described above. The flap system consisted of three parts: inboard USB flaps with a smooth, continuous surface, mid-span double-slotted flaps, and an essentially unflapped outboard section. Figure 7-9 illustrates the model, and detailed data was given previously in Table 7-1. 


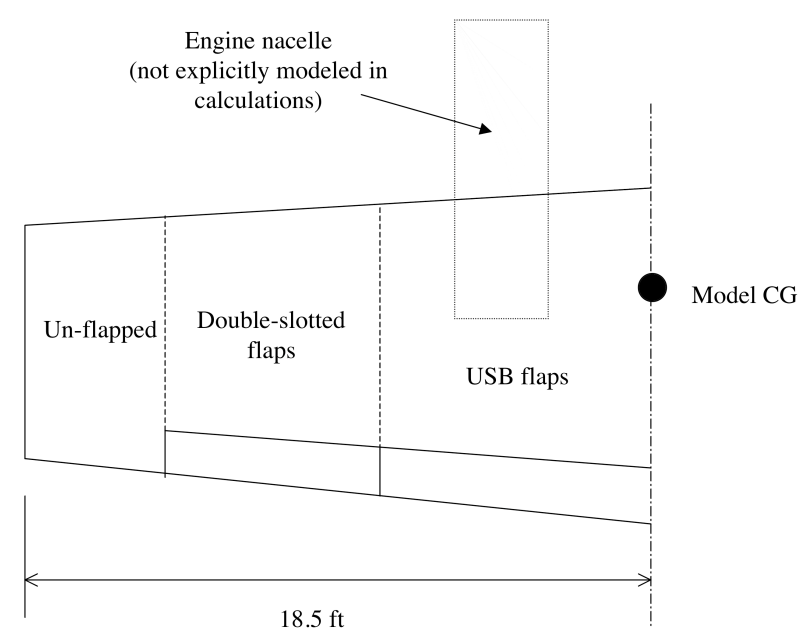

Figure 7-9: Wing planform used in experiments of Ref 54.

Once again, the correlating parameter used by the authors was the total thrust coefficient. Following equations (7-1) and (7-2), the corresponding 2-D blowing coefficient over the USB section will clearly be higher due to the smaller span of blowing. This trade-off between section $C_{j}$ and blown-span is of central importance in USB aircraft design. The propulsion system simulator used in the experiments consisted of 4 small fans in a common nacelle. These fans simulated a turbofan with a mixed-flow exhaust nozzle. All comparisons with the current method used data for a rectangular nozzle with an aspect ratio of 4 . The outboard sectional data and mid-span flapped sectional data were taken from Abbott and Von Doenhoff ${ }^{(53)}$. The flapped data was approximated under the assumption that the flaps add relatively constant increments, and that the leading edge is well protected from stall.

The current method was used to make predictions at total thrust coefficients of $C_{T}=1.0$ and 2.0 and flap settings (USB and double-slotted) of 0, 20, 40, and 60 degrees. Each configuration shows polars over an angle of attack range of 0 to 25 degrees. Although higher thrust coefficients were tested in the experiment, these thrust coefficients corresponded to equivalent 2 -D blowing coefficients that were in the upper range $\left(C_{j} \approx 9\right.$ - 
10) where the current analytical method begins to break down. Figures 7-10 through 713 show the lift curve comparisons for each flap setting.

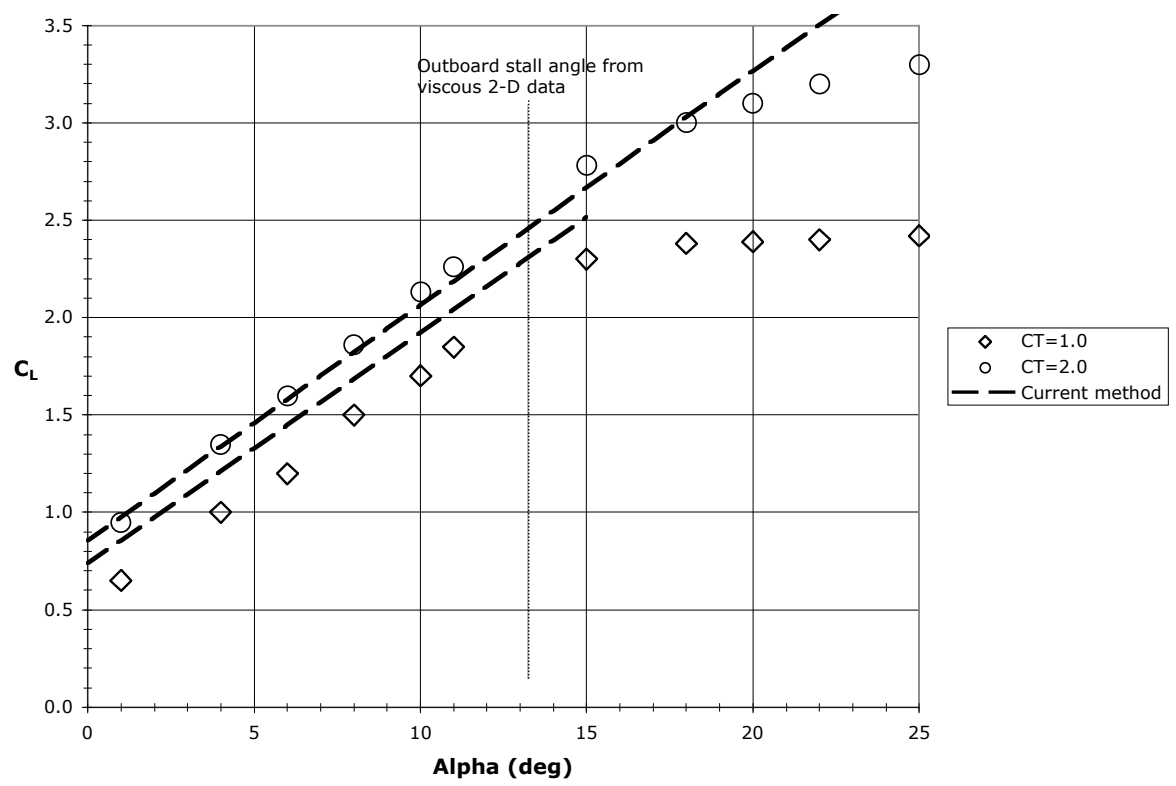

Figure 7-10: $C_{L}$ variation with Alpha for 0 deg flaps at 2 thrust settings

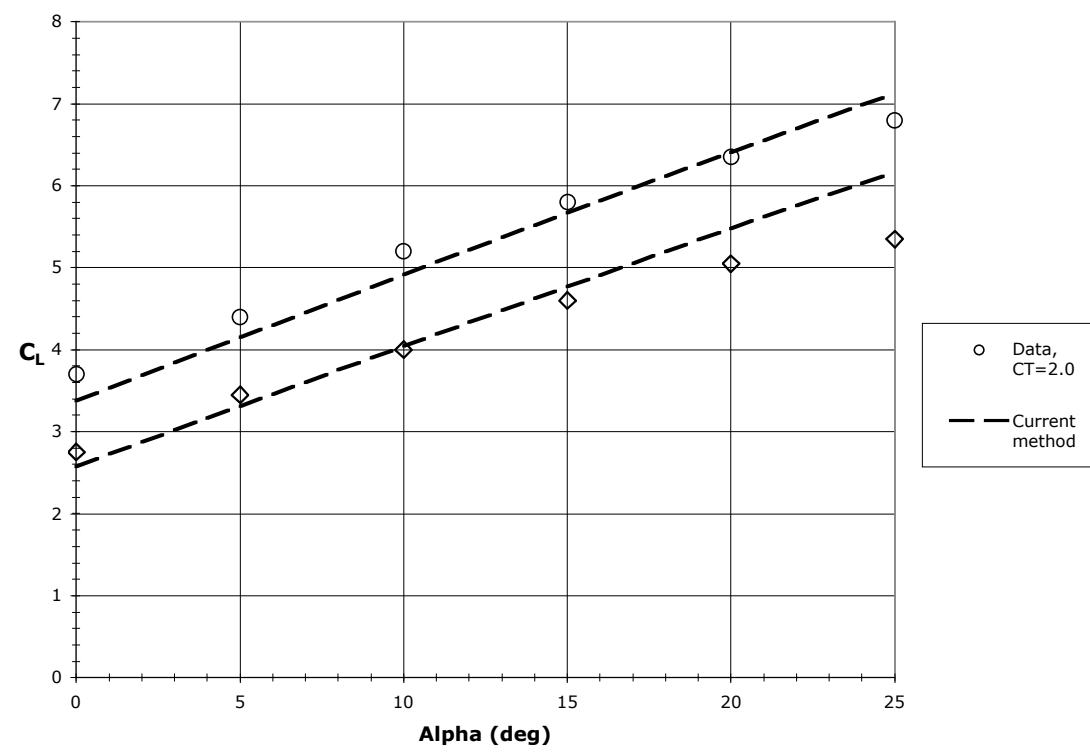

Figure 7-11: $C_{L}$ variation with Alpha for 20 deg flaps at 2 thrust settings 


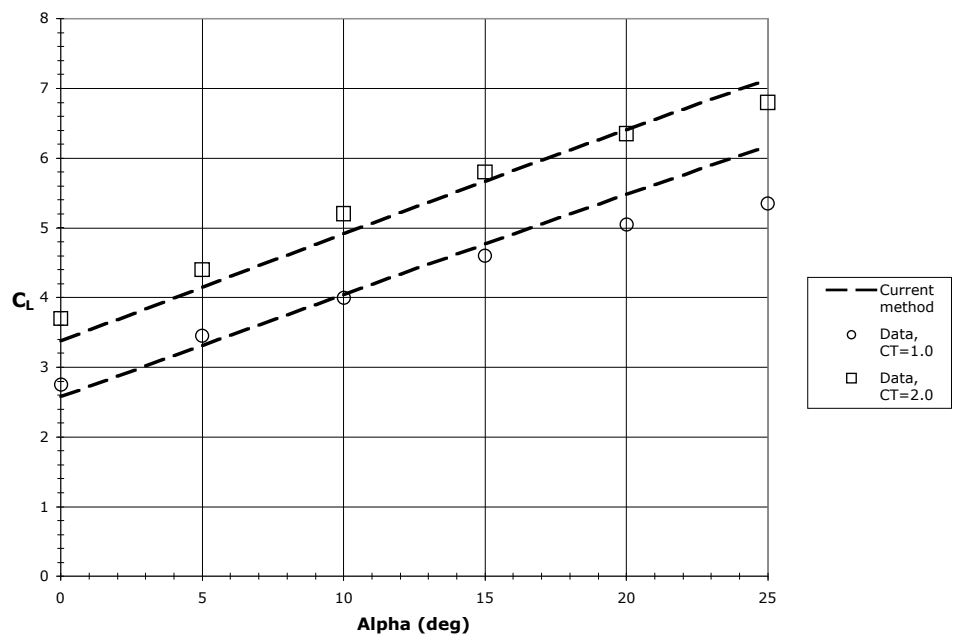

Figure 7-12: $C_{L}$ variation with Alpha for 40 deg flaps at 2 thrust settings

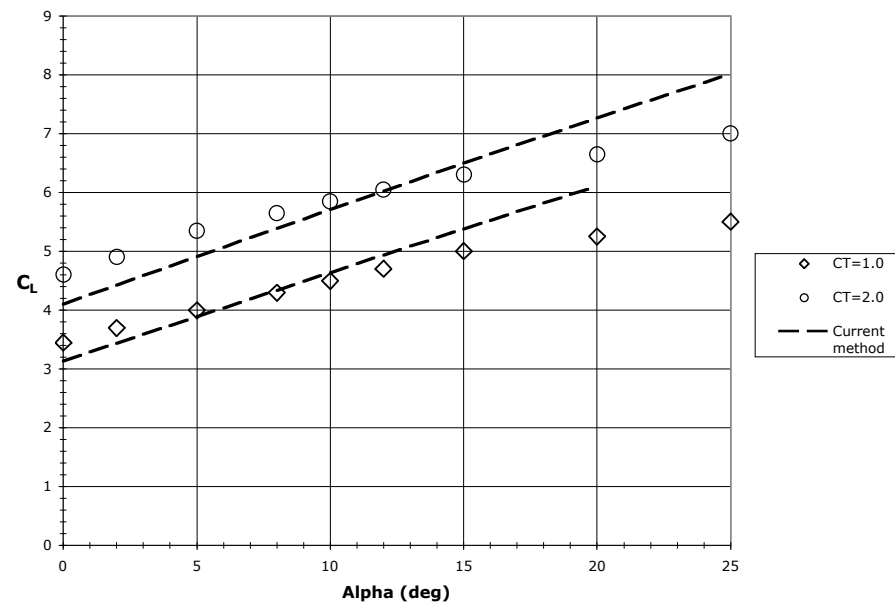

Figure 7-13: $C_{L}$ variation with Alpha for 60 deg flaps at 2 thrust settings

We see that the lift coefficients match the experimental data very well, with the unusual exception for $C_{T}=1.0$ at 0 deg flap deflection. Also in Figure 7-10, note that for small thrust coefficients, the stall angle of the wing without leading edge protection is governed by the stall angle of the outboard section. For large flap deflections the lift curve slopes begin to show more of a non-linear behavior, though the classic stall pattern is not seen. The current method captures the correct magnitude (within 10\% of experiment) and matches the lift curve slope at small angles of attack. 
Next, examine the drag coefficient predictions with experiment. As described in section 6.3, the high-lift module encounters numerical problems when dealing with rapid changes in the sectional properties across the span, and a twin-engine configuration will have such changes. To counter the additional induced drag that is predicted, a small study was done on the experimental data to determine the general values of the span efficiency factor, $e$. The parabolic form of the drag equation is:

$$
C_{D}=C_{D, M}+\left(\frac{1}{\pi \mathrm{AR} e}\right)\left(C_{L}-C_{L, M}\right)^{2}
$$

where $\mathrm{AR}$ is the aspect ratio and $C_{D, M}$ and $C_{L, M}$ are the drag and lift coefficients at the parabola vertex.

Using methods described by Recktenwald ${ }^{(56)}$, a short non-linear regression program was written in MATLAB as part of the current work. Experimental data sets were then input and the span efficiency factor determined. It was found that the magnitude of $e$ varied between about 0.50 and 0.70 with most cases being around 0.63 for the experimental cases. There also appeared to be an increase in the efficiency factor with increasing blowing coefficient. This effect was also described by Whitten ${ }^{(57)}$, who says regarding the drag with increasing power setting, “...an improvement in polar shape is obtained along with an increase in minimum drag". The efficiency factors output from the high-lift module were typically around $e=0.35$ and varied very little with power setting.

Using the non-linear regression program, values of $e, C_{D, M}$ and $C_{L, M}$ were obtained for the output drag polars, and then equation (7-3) was used with an improved value for $e$ to basically "reconstruct" a polar. The resulting drag polars had a more appropriate shape and much better agreement in magnitude. An example of this change is shown in Figure 
$7-14$, where the red line represents the values originally output by the high-lift module and the black line represents the corrected values.

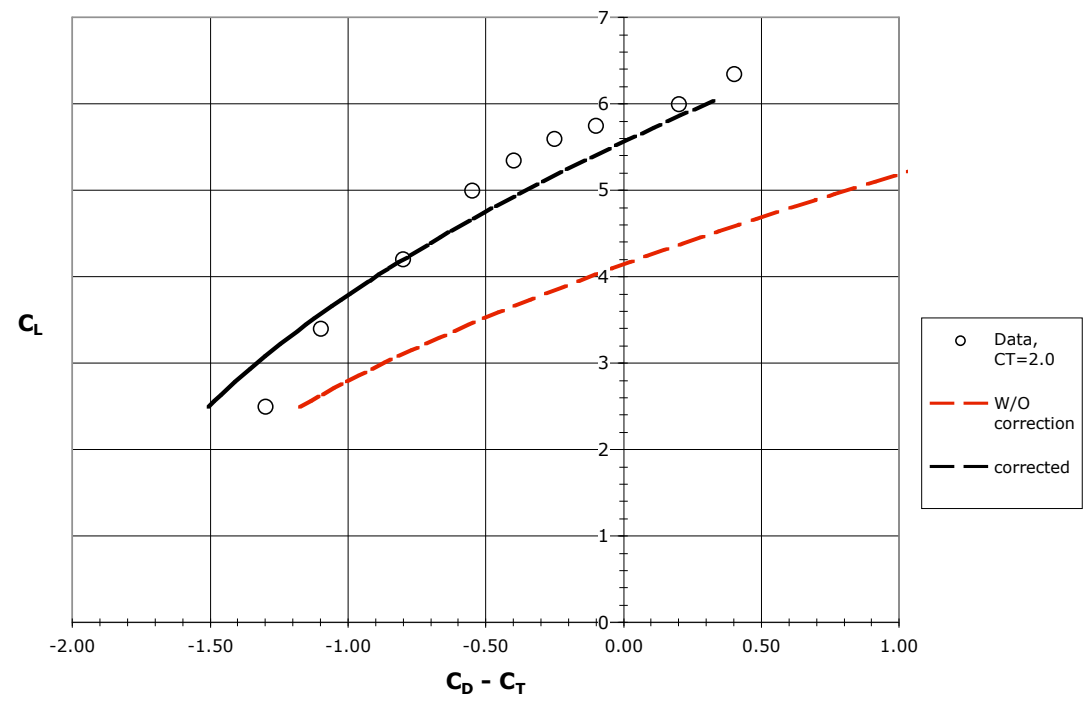

Figure 7-14: Example of induced drag correction for thrust-plus-drag polars

Figures 7-15 through 7-18 show the thrust-plus-drag polars for various flap settings:

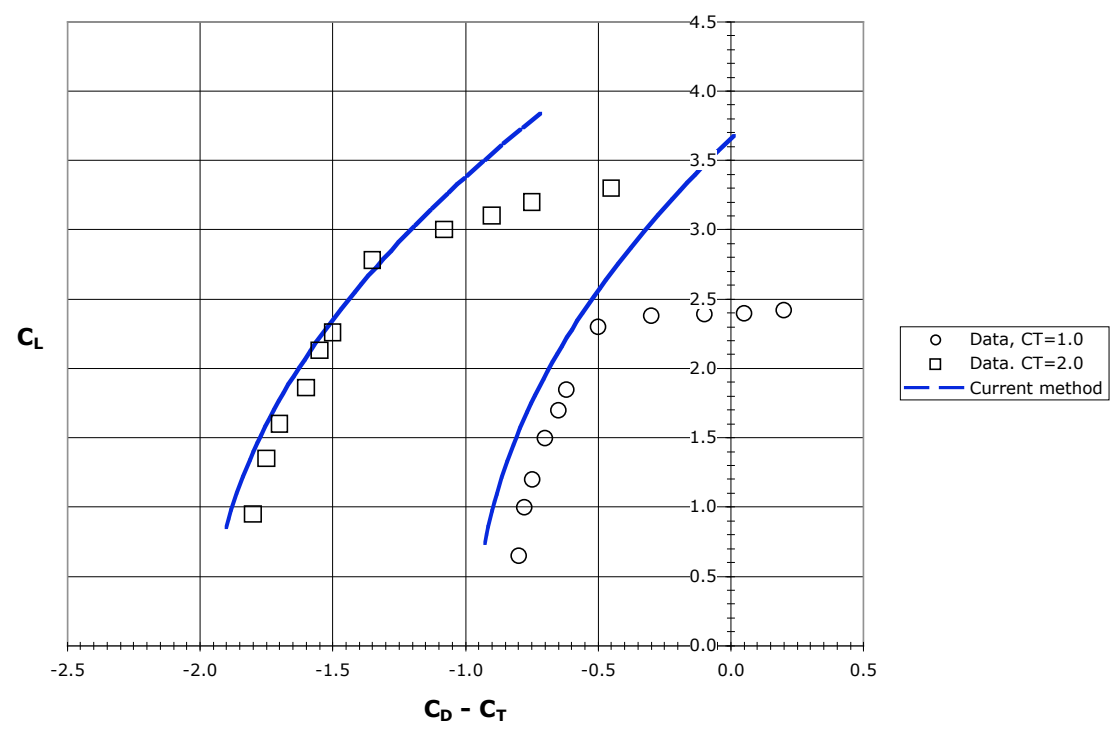

Figure 7-15: Thrust-plus-Drag polar for 2 power settings at 0 deg flaps 


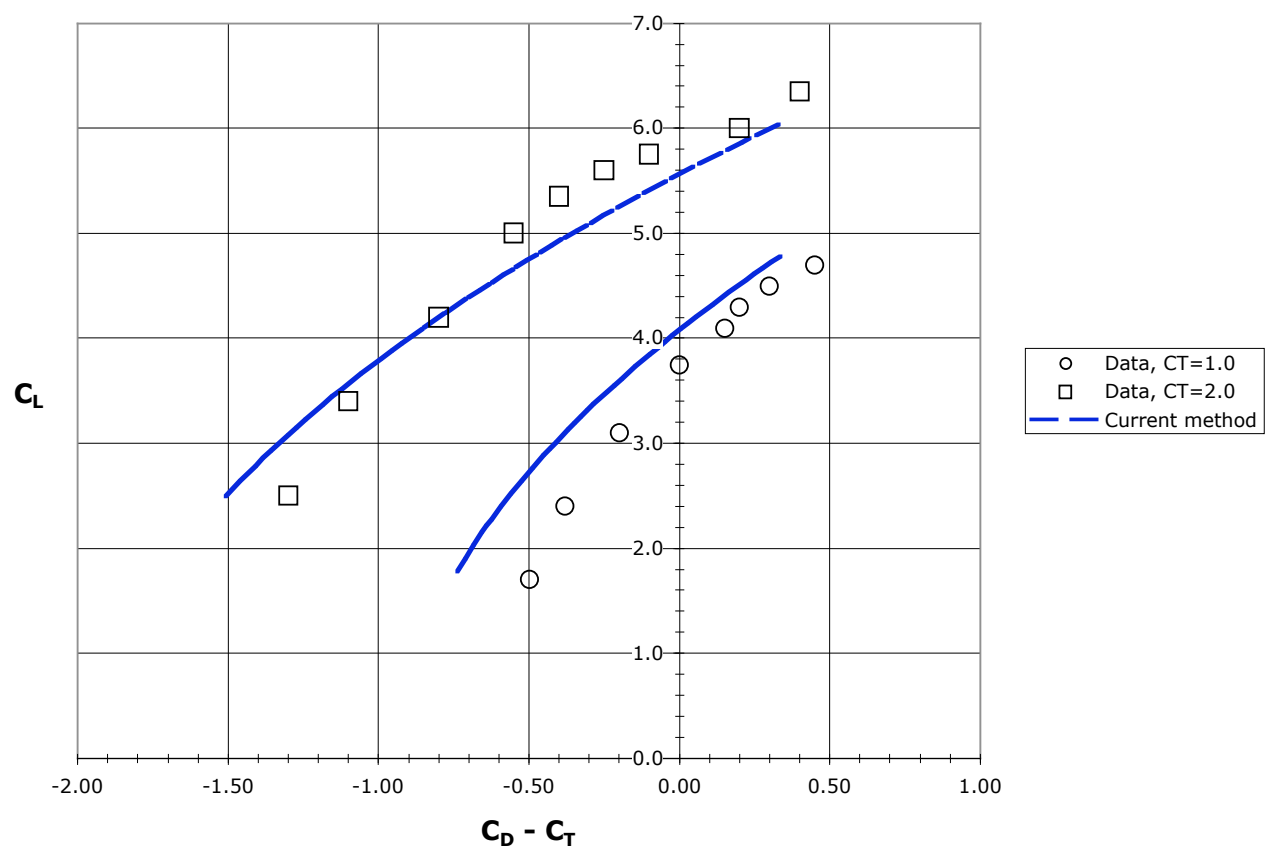

Figure 7-16: Thrust-plus-Drag polar for 2 power settings at 20 deg flaps

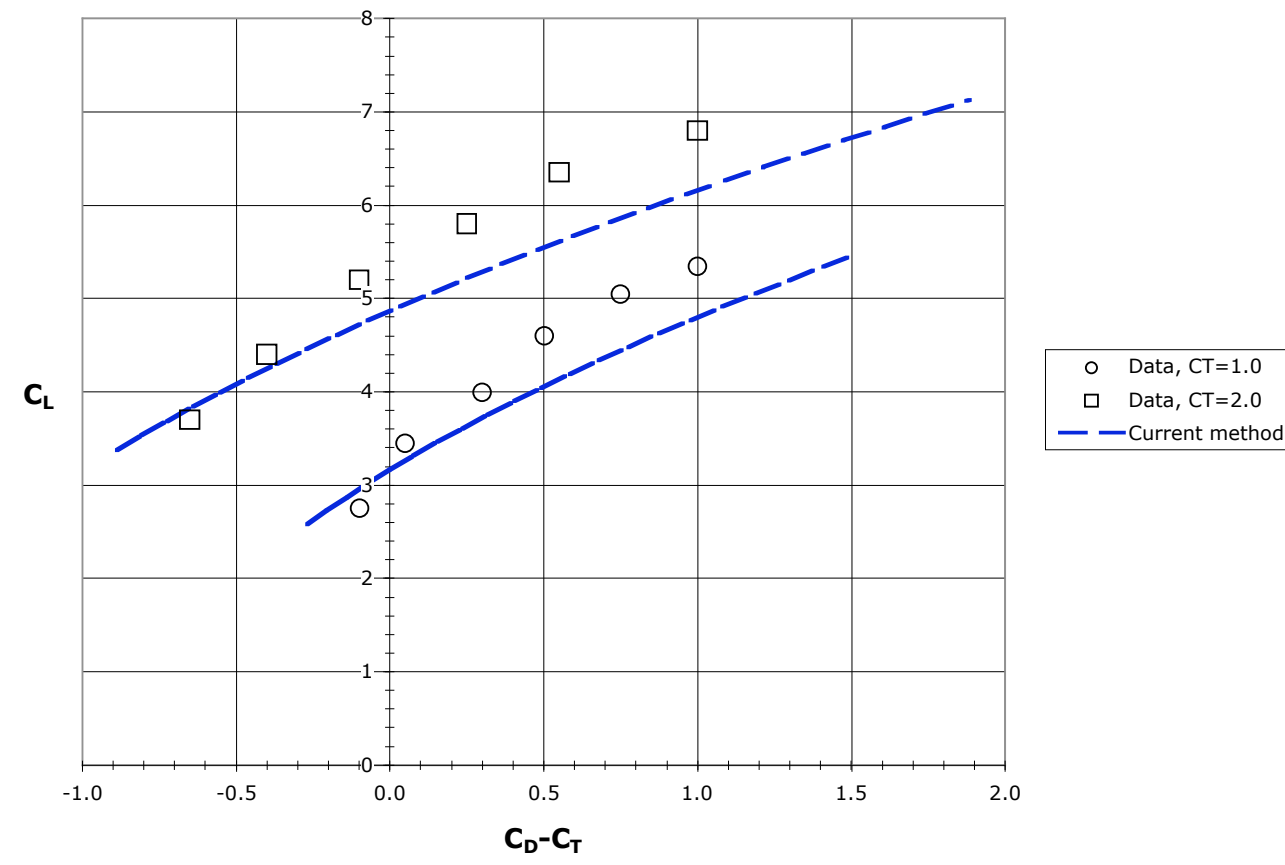

Figure 7-17: Thrust-plus-Drag polar for 2 power settings at 40 deg flaps 


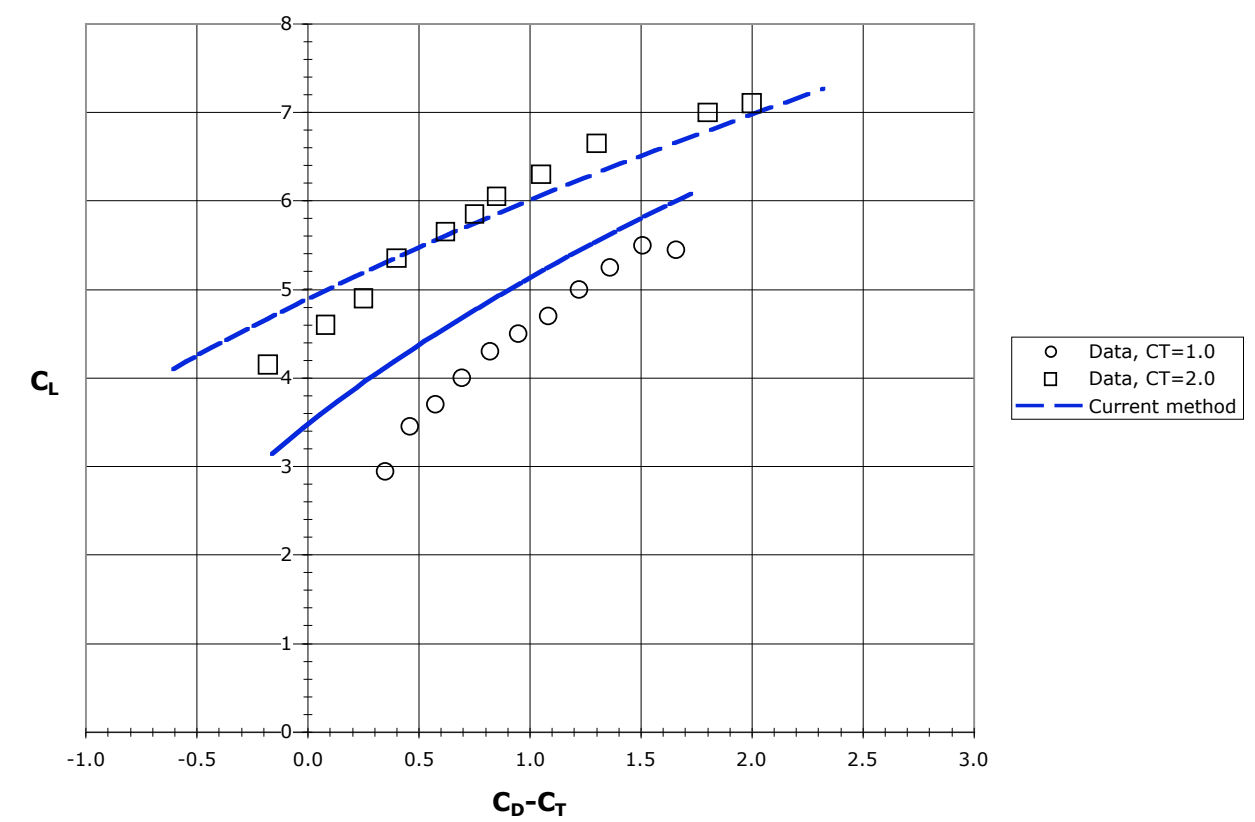

Figure 7-18: Thrust-plus-Drag polar for 2 power settings at 60 deg flaps

The previous figures show that the corrections provide a much more reasonable estimate for the drag coefficients. Such corrections would not be needed if the numerical problems encountered by the high-lift module were corrected. The drag predictions, as expected, begin to deviate from exact values as we go to high flap deflections. With a more accurate surface geometry model or more sophisticated estimates of leading edge suction values, we anticipate that the drag would be predicted more accurately.

Lastly, the pitching moment predictions are shown in Figures 7-19, 7-20, and 7-21 for flap settings of 0,20 , and 40 degrees respectively. 


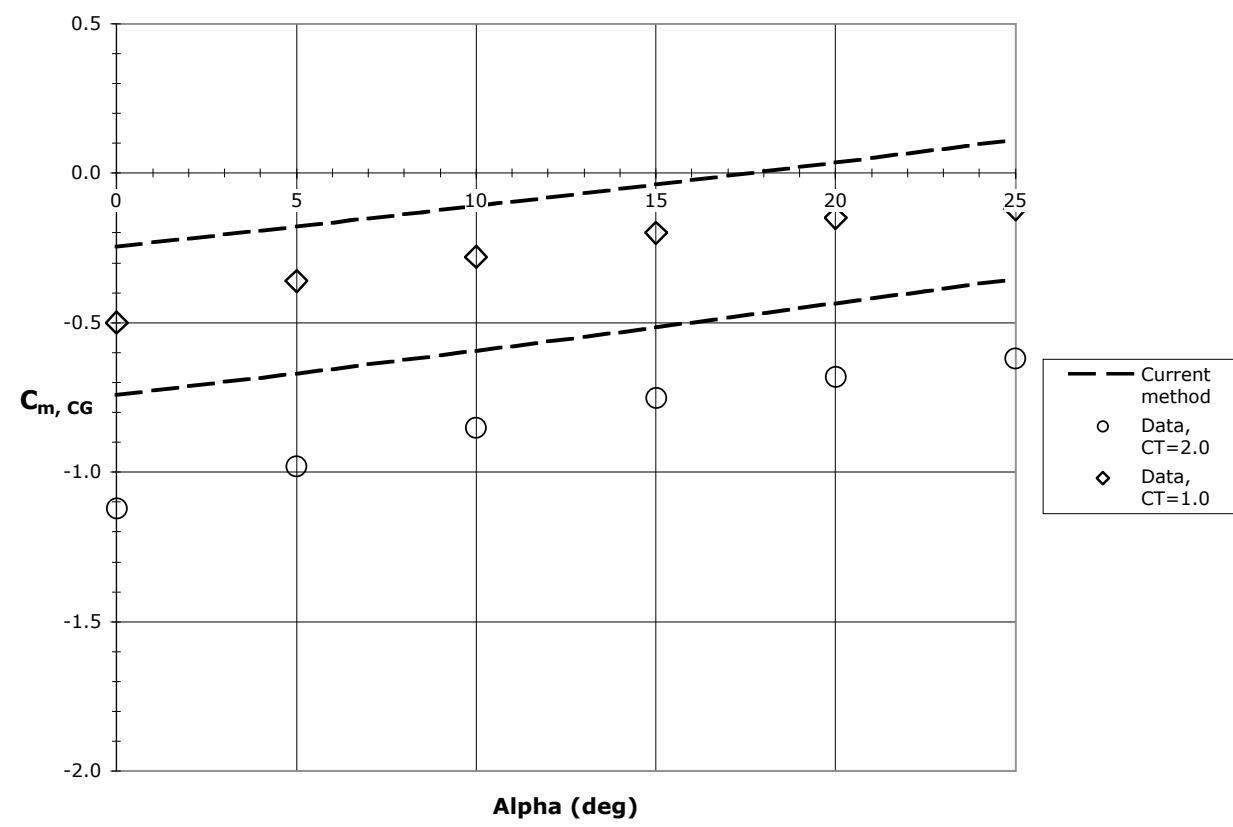

Figure 7-19: Pitching moment about the aircraft C.G. vs. Alpha for 0 deg flaps

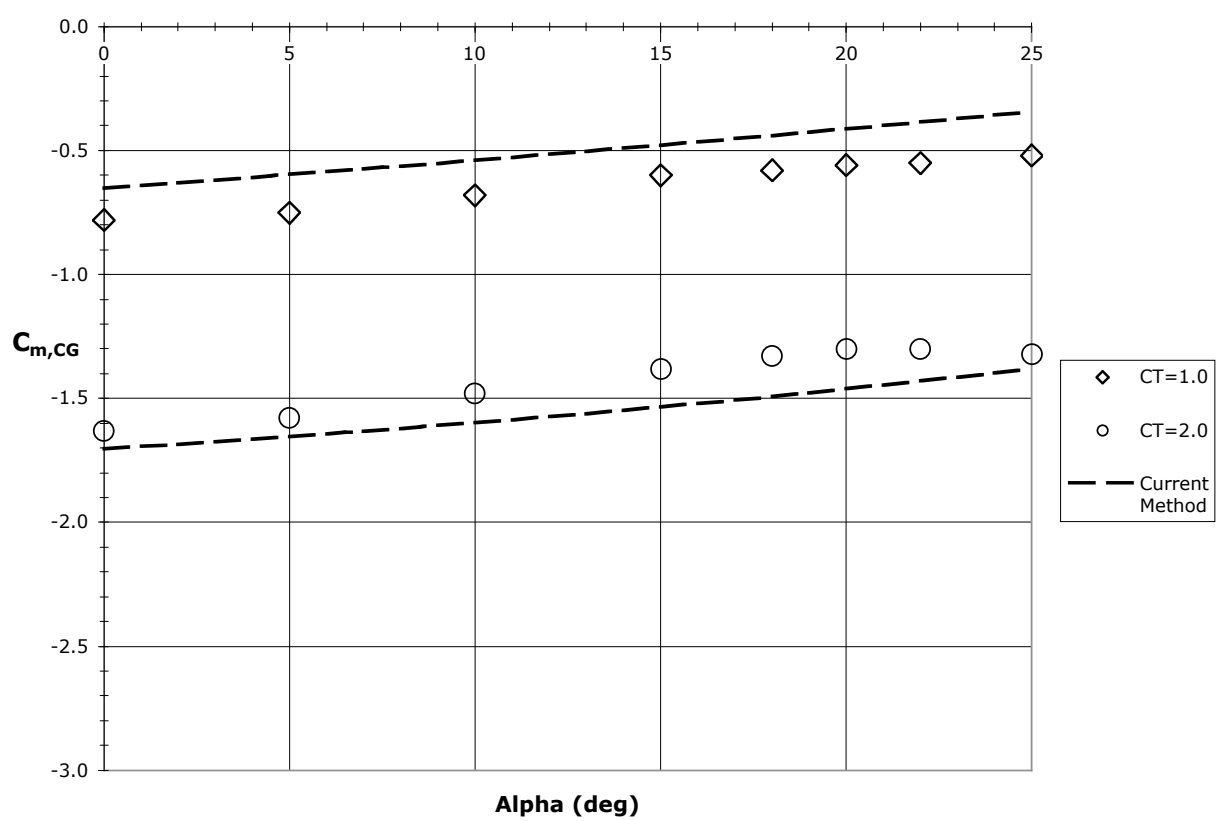

Figure 7-20: Pitching moment about the aircraft C.G. vs. Alpha for 20 deg flaps 


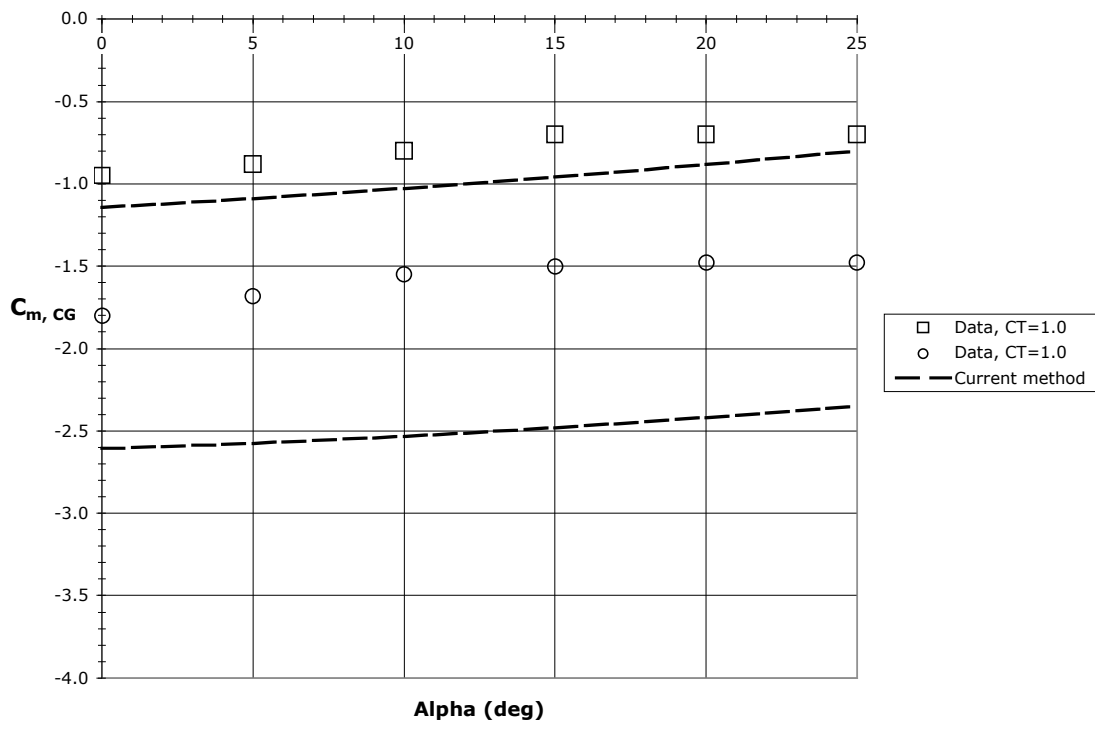

Figure 7-21: Pitching moment about the aircraft C.G. vs. Alpha for 40 deg flaps

In all cases the pitching moment slope about the aircraft C.G. is captured well.

Prediction of $C_{m o}$ is much more difficult and the current analysis begins to overpredict the pitching moment as flap deflection becomes large. It is important to note that the vertical location of the wing was not accounted for in the previous plots of pitching moment.

Due to the relatively high drag levels from USB, a high-wing configuration will get some "nose-down" pitching moment relief by the "nose-up" pitching moment caused by the drag on a high wing. However, since the internal drag calculations of the High-Lift module were inaccurate for this configuration (see above post-processing corrections), including the drag values in the pitching moment calculation was deemed impractical.

\subsection{3 - Case 3: Small-scale, Four-engine configuration (TN D-7399)}

This work done by Phelps and Smith ${ }^{(55)}$ in 1973 recorded the longitudinal and lateral aerodynamic characteristics of a small-scale, four-engine, swept-wing USB aircraft. The importance of this study was that it provided force and moment data for engine-out cases, which are important design points for powered-lift aircraft. The wing consisted of 
inboard USB flaps, which covered about half of span, and outboard double-slotted flaps. Figure 7-22 shows a sketch of the model's wing, and other details were given in Table $7-1$.

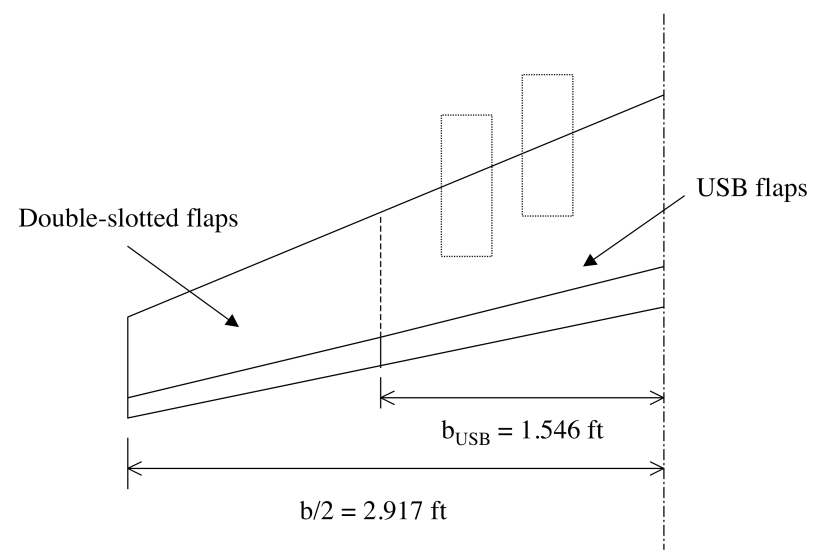

Figure 7-22: Wing planform used in Ref 55

The total thrust coefficient was the correlating parameter used in the study. Test points were taken at $C_{T}=1.08$ and 2.16 which correspond to blowing coefficients, $C_{j}=$ 1.805 and 3.621. The outboard flapped sectional data was again approximated using data from refs. 52 and 53. Comparisons were done for the thrust coefficients listed above at 0 and 35 deg flaps (USB and double-slotted) over an angle of attack range of -5 to 25 degrees.

Begin with the 0 deg flap case. Figures 7-23 through 7-25 show the lift, drag, and pitching moment coefficients for $C_{T}=2.16$. Note that the $C_{T}=1.08$ case was not run for this flap setting. 


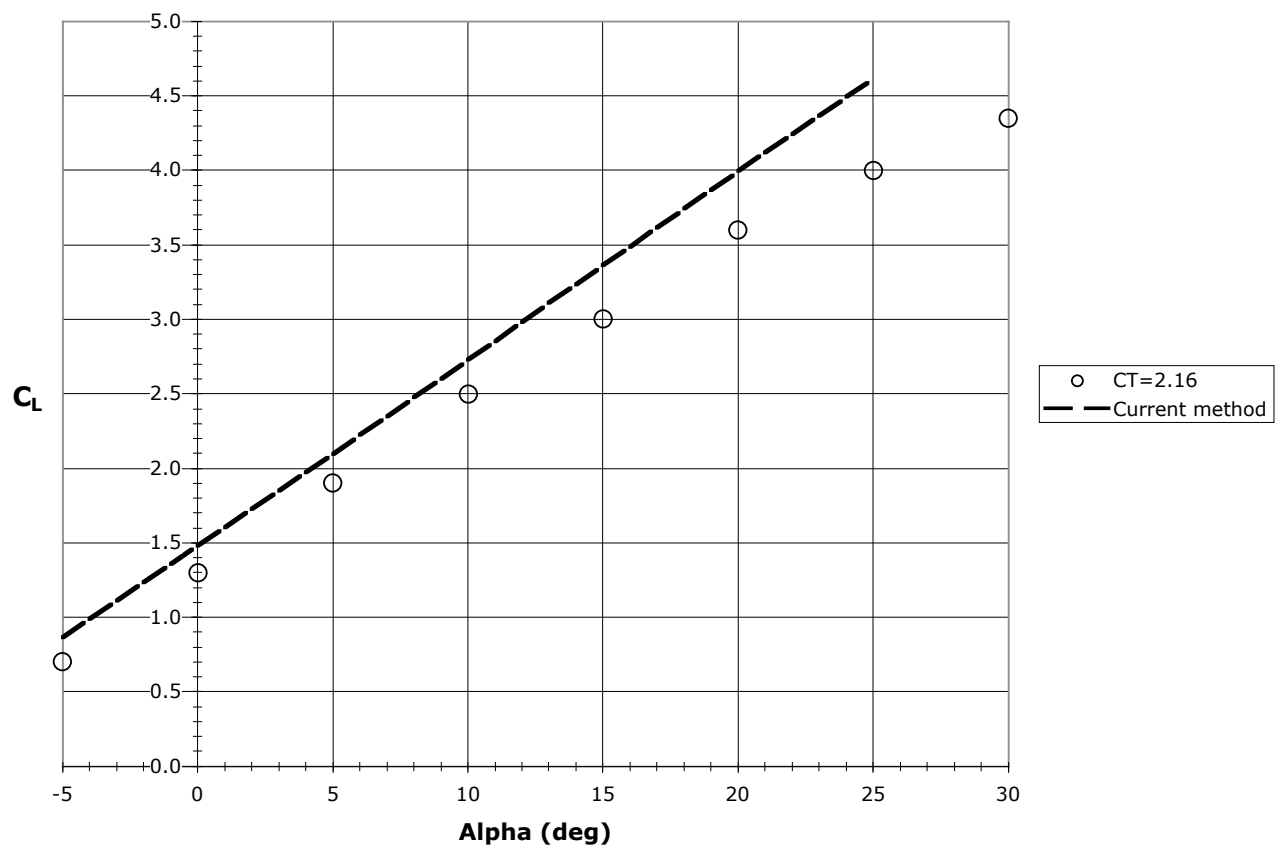

Figure 7-23: $C_{L}$ variation with Alpha for 0 deg flaps at $C_{T}=2.16$

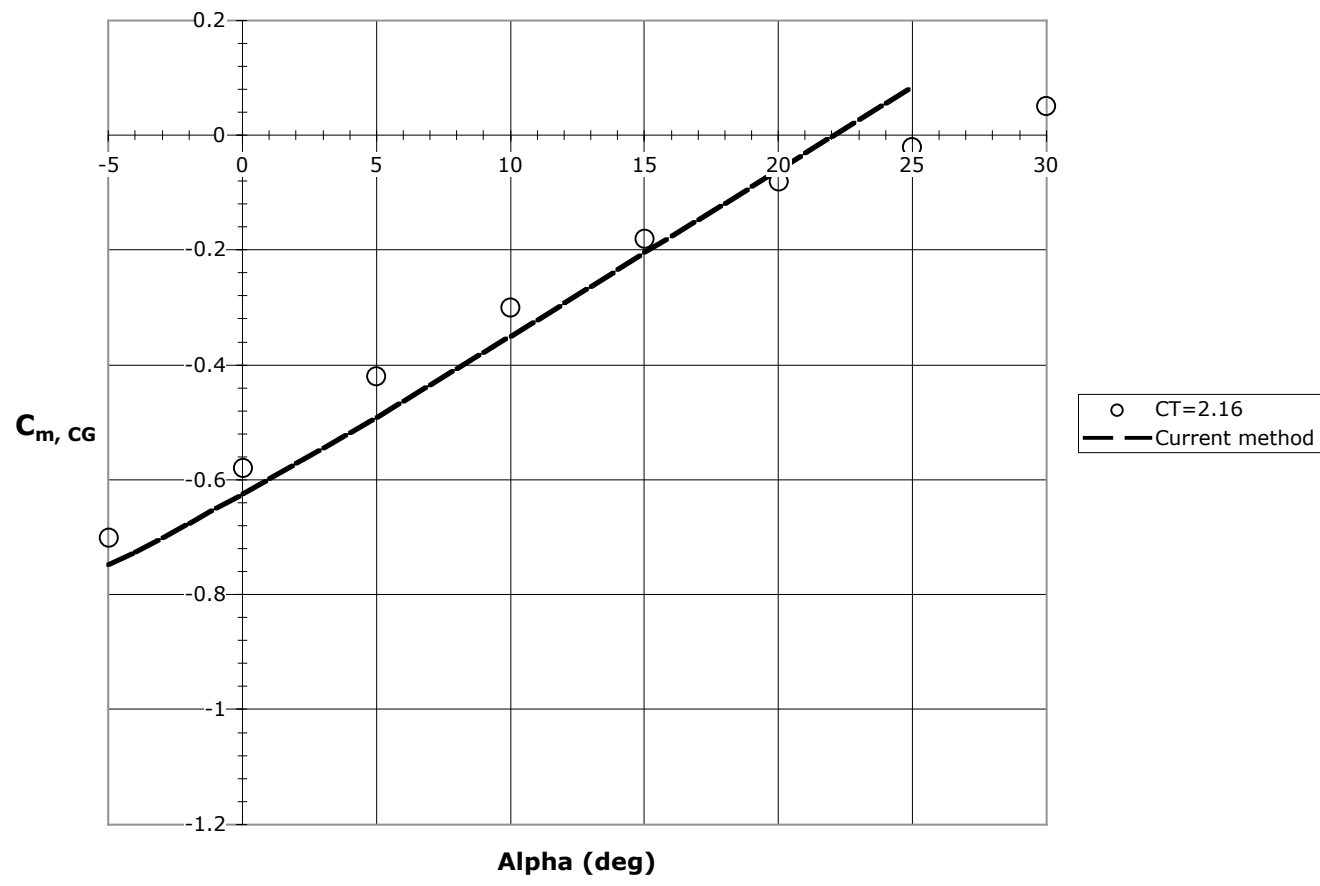

Figure 7-24: Pitching moment about the aircraft C.G. vs. Alpha for 0 deg flaps at $C_{T}=2.16$ 


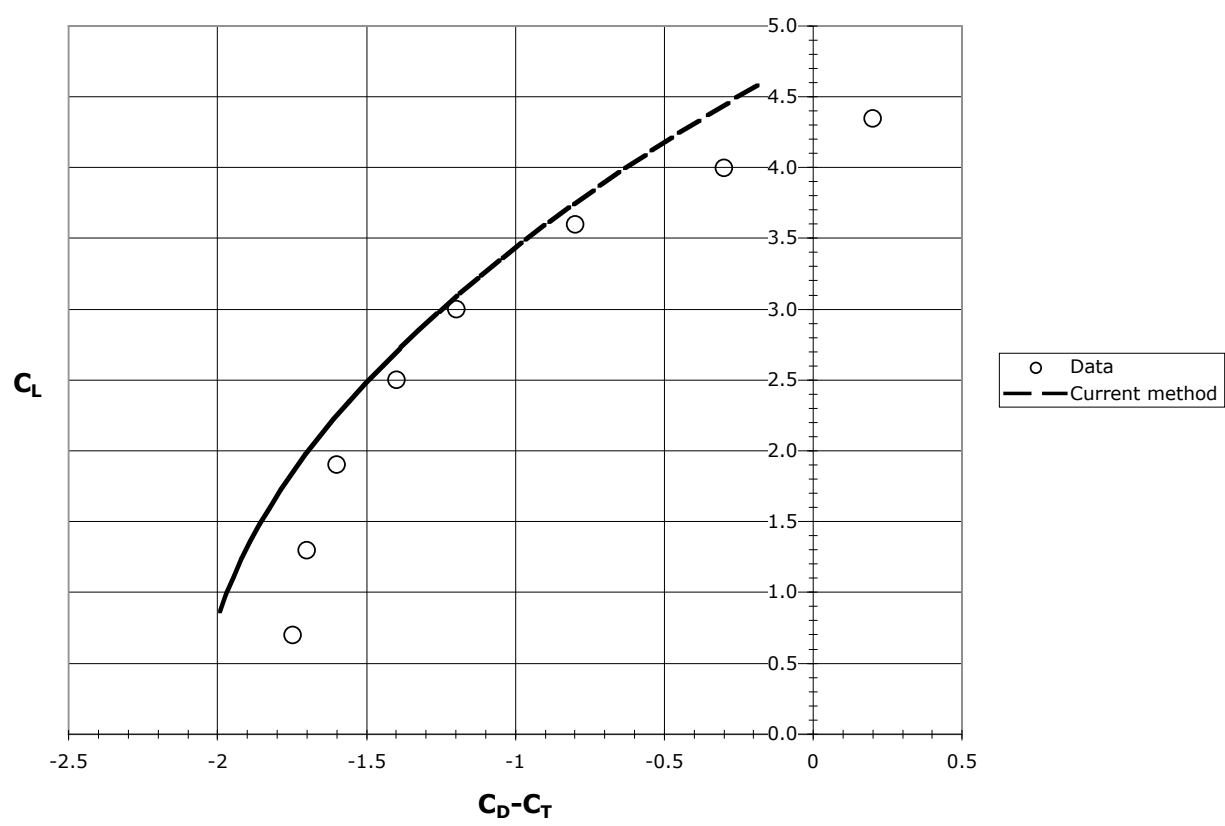

Figure 7-25: Thrust-plus-Drag polar for 0 deg flaps at $C_{T}=2.16$

From the previous figures, the current method clearly agrees well with the experimental data both in magnitude and slope $\left(C_{L \alpha}\right.$ and $\left.C_{m \alpha}\right)$. Also note that the drag polar required no corrections since the model used a 4-engine configuration, which spreads the USB loading over a greater portion of the wing. The output values of the span efficiency factor, $e$, were similar to the values obtained by non-linear regression of the experimental data.

Now examine the comparisons done with the trailing edge devices set to 35 degrees deflection. Figures 7-26 through 7-28 show the lift, drag, and pitching moment coefficients at different thrust settings. 


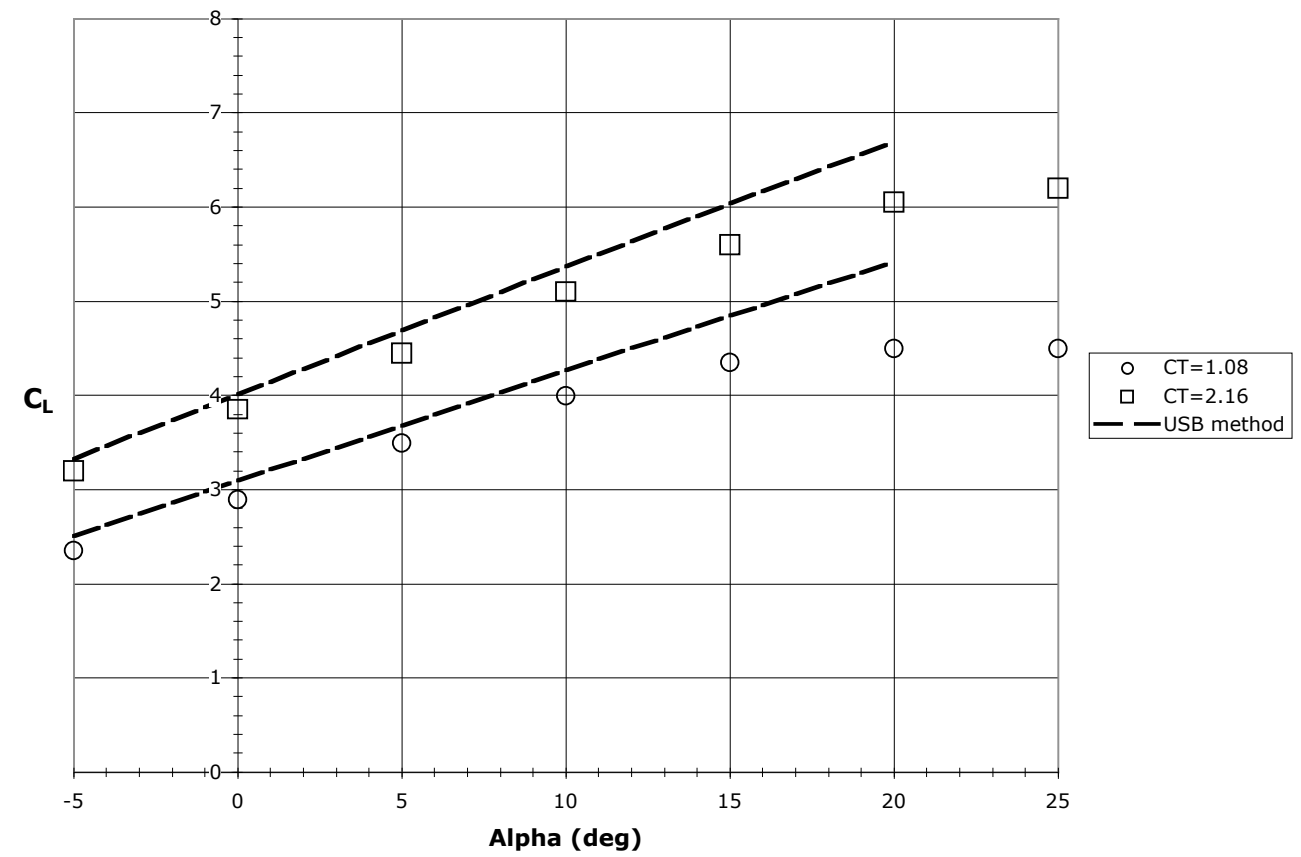

Figure 7-26: $C_{L}$ variation with Alpha for 35 deg flaps at 2 thrust settings

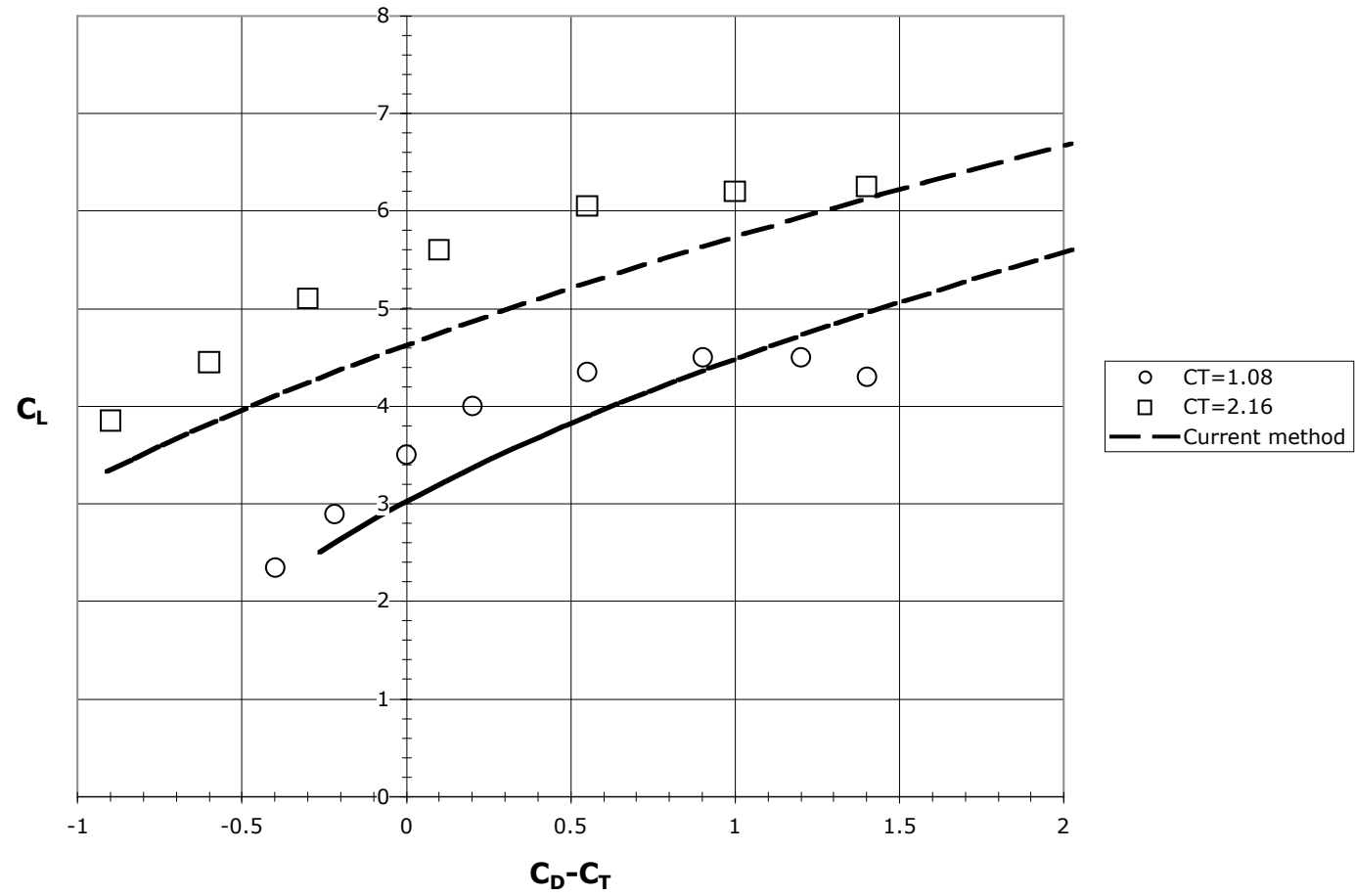

Figure 7-27: Thrust-plus-Drag polars for 35 deg flaps at 2 thrust settings 


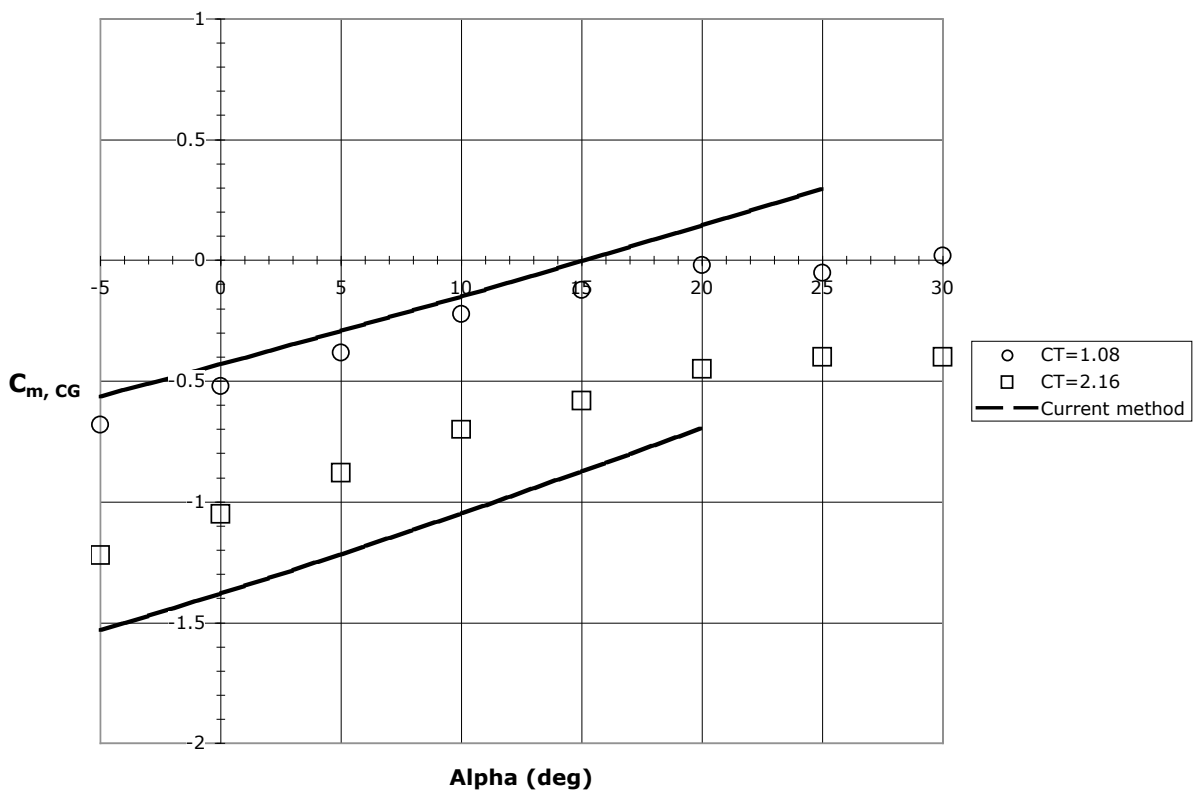

Figure 7-28: Pitching moment about the aircraft C.G. vs. Alpha for 35 deg flaps at 2 thrust settings

The lift magnitude and slope is in good agreement with experiment. While the pitching moment magnitude begins to be over-predicted for high thrust coefficients, $C_{m \alpha}$ is captured very well in the unstalled region. At the higher flap setting, the drag polars begin to lose some accuracy. This is believed to be due to the incomplete model of the flap geometry in the 2-D calculations, and the beginning of the spanload oscillation effects from the High-Lift module.

\subsection{YC-14 Data}

The Boeing YC-14 was an experimental aircraft built in 1976 by the Boeing Company to demonstrate the STOL capabilities associated with USB aircraft ${ }^{(5)}$. It is, to this day, one of the few successful flight demonstrators of USB technology. As such, a large amount of information is available about its wind tunnel testing and flight testing. Given the information about the wing layout, engine size and type, and flap geometry, a 
good model could be created for our analysis. This situation is more representative of the type and amount of information that will be available during the preliminary design phases.

The YC-14 was a high-wing, twin-engine aircraft with an unswept wing. The exhaust nozzles were carefully contoured to provide a relatively thin jet sheet without excessive nozzle losses. The wing consisted of an inboard USB flap, mid-span double-slotted flaps, and an outboard control section. Figure 7-29 shows a drawing of the wing planform.

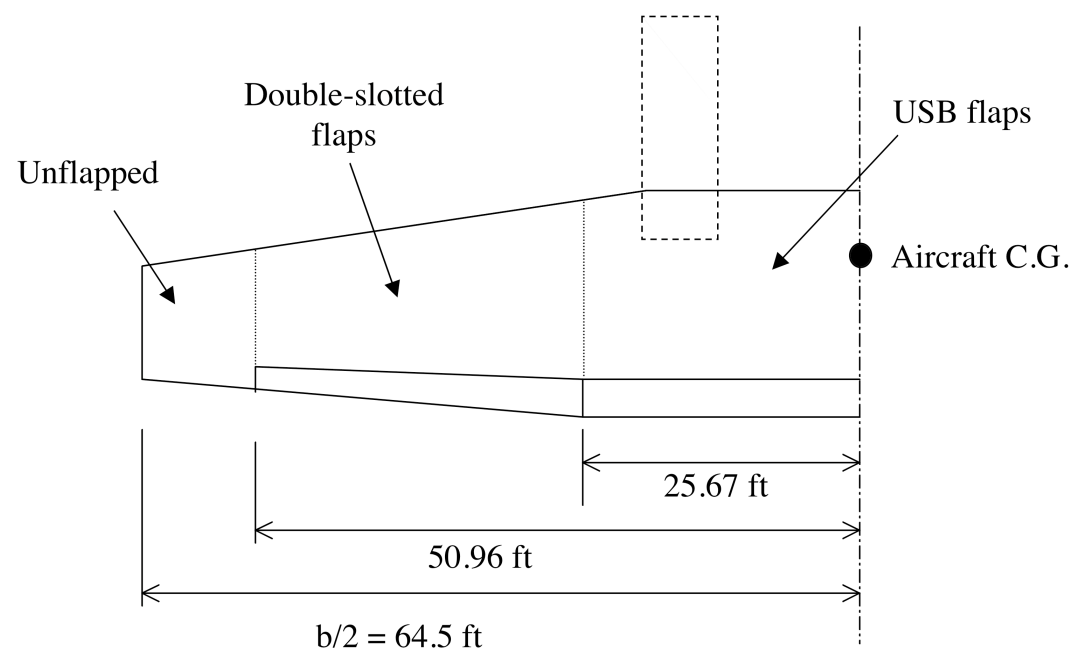

Figure 7-29: Wing planform for the Boeing YC-14

Table 7-2 lists other parameters used to perform calculations

Table 7-2: YC-14 data from Ref 5

\begin{tabular}{|l|l|}
\hline \multicolumn{1}{|c|}{ Parameter } & \multicolumn{1}{c|}{ Value used in calculations } \\
\hline Aspect Ratio & 9.44 \\
\hline Wing Area & $1762.4 \mathrm{ft}^{2}$ \\
\hline Wing Taper & 0.35 \\
\hline TE flap chord ratio $\left(\mathrm{c}_{\mathrm{f}} / \mathrm{c}\right)$ & 0.30 \\
\hline Airfoil section type & supercritical \\
\hline Avg. chord for USB section & $17.5 \mathrm{ft}$ \\
\hline Airfoil TE angle & $13.75 \mathrm{deg}$ \\
\hline Nozzle exit area & $17.2 \mathrm{ft}^{2}$ \\
\hline Nozzle height & $2.0 \mathrm{ft}$ \\
\hline $\mathrm{b}_{\text {USB }}$ & $25.67 \mathrm{ft}$ \\
\hline t/c @ wing root & 0.16 \\
\hline Spanwise location of engine & $0.208 \mathrm{~b} / 2$ \\
\hline Mach number for STOL & 0.08 \\
\hline Engine size and type & $(2) \mathrm{GE} \mathrm{CF6-50} \mathrm{(48,000} \mathrm{lbs} \mathrm{ea)}$ \\
\hline
\end{tabular}


Information about the exit conditions for the CF6 engines is readily available. The flap geometry was approximated using information from Ref. 7. Based on this information, the extension, deflection, and curvature of the aft portion of the USB sections could be simulated well. Figure 7-30 demonstrates the discretized surface (see section 4.3.2) for flap deflections of 0 to 60 degrees. The $x$ and $y$ axes are length scales, with the origin representing the $x$-location of the nozzle exit on the chord line.

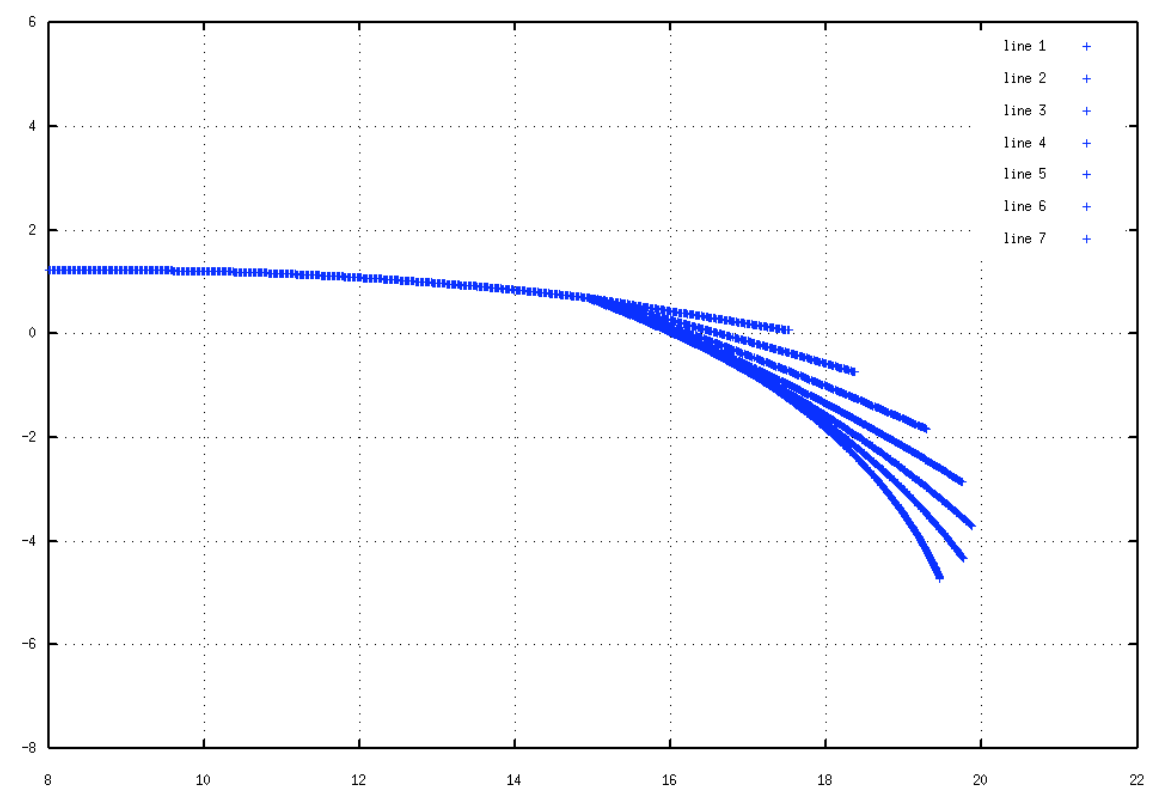

Figure 7-30: Upper surface profiles for various flap deflections

Data was given in reference 5 for the takeoff and landing configurations of the YC-

14. The takeoff configuration used $20 \mathrm{deg}$ USB flaps while the landing configuration used 60 deg USB flaps. Data was given for 2 different thrust settings in the 2 configurations over a range of alpha from -5 to 35 degrees. Figures 7-31 and 7-32 present the comparison of the experimental data with predictions using the current method for the takeoff configuration. 


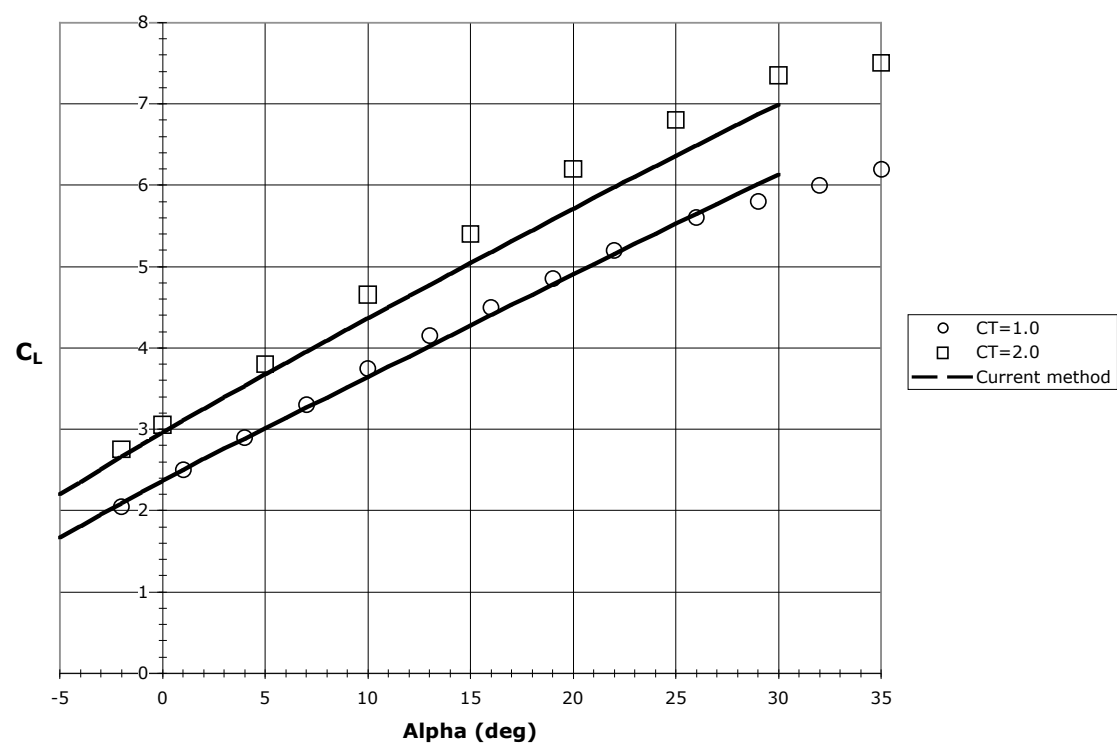

Figure 7-31: $C_{L}$ variation with Alpha for takeoff configuration at 2 thrust settings

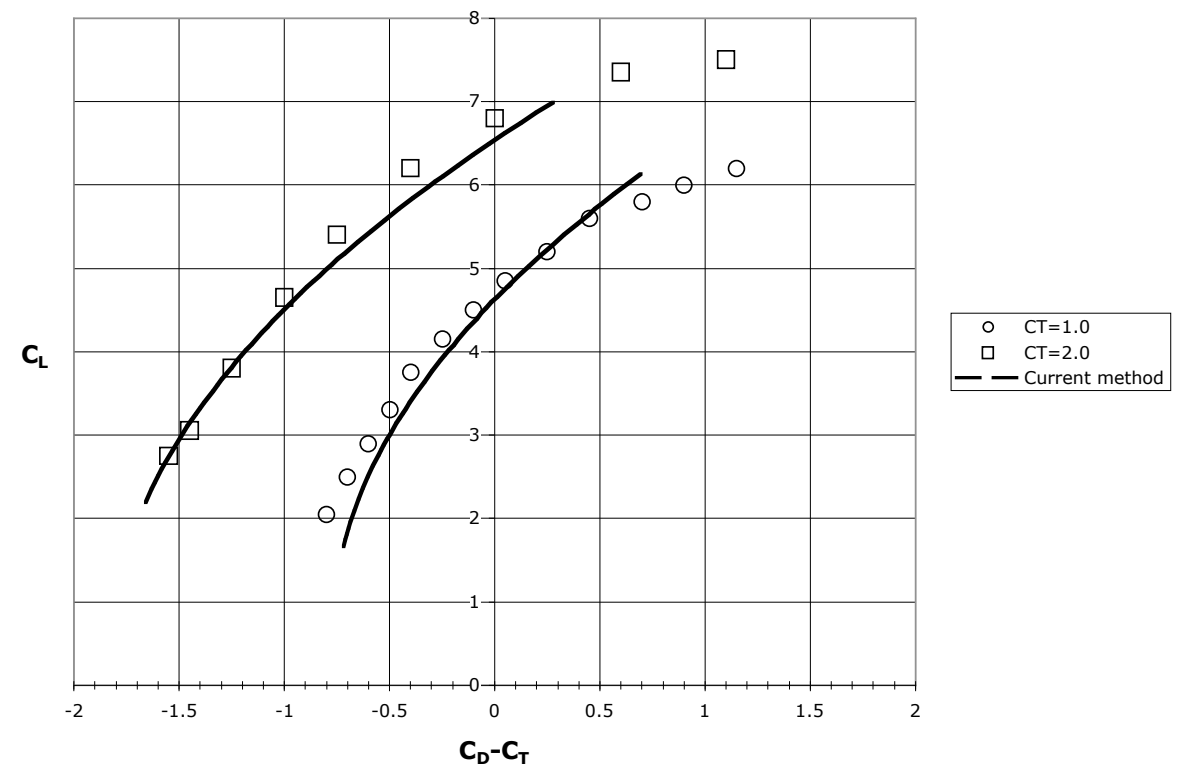

Figure 7-32: Thrust-plus-Drag polars for takeoff configuration at 2 thrust settings

The current method agrees with experimental data very well. No pitching moment data was provided (but we anticipate that these values would show similar agreement). It is also important to note that the drag values were again "corrected" to eliminate the extra induced drag. This was anticipated given the twin-engine configuration coupled with 
high blowing coefficients, and the polars were modified in the same manner as those in section 7.1.2.

The comparisons for the landing configuration are shown in Figures 7-33 and 7-34. The higher thrust setting was found to have relatively poor agreement with experimental data, and is not shown. It is believed that for these thrust settings the use of vortex generators on the flap surface was necessary to maintain turning performance. Vortex generators are small plates that can be used to introduce turbulence in the jet flow. This turbulence increases the turning angle but has an adverse effect on the aft pressures and turning efficiency. The effects of these generators is not modeled in the 2-D calculations, and are the likely cause of the discrepancy at $C_{T}=2.0$.

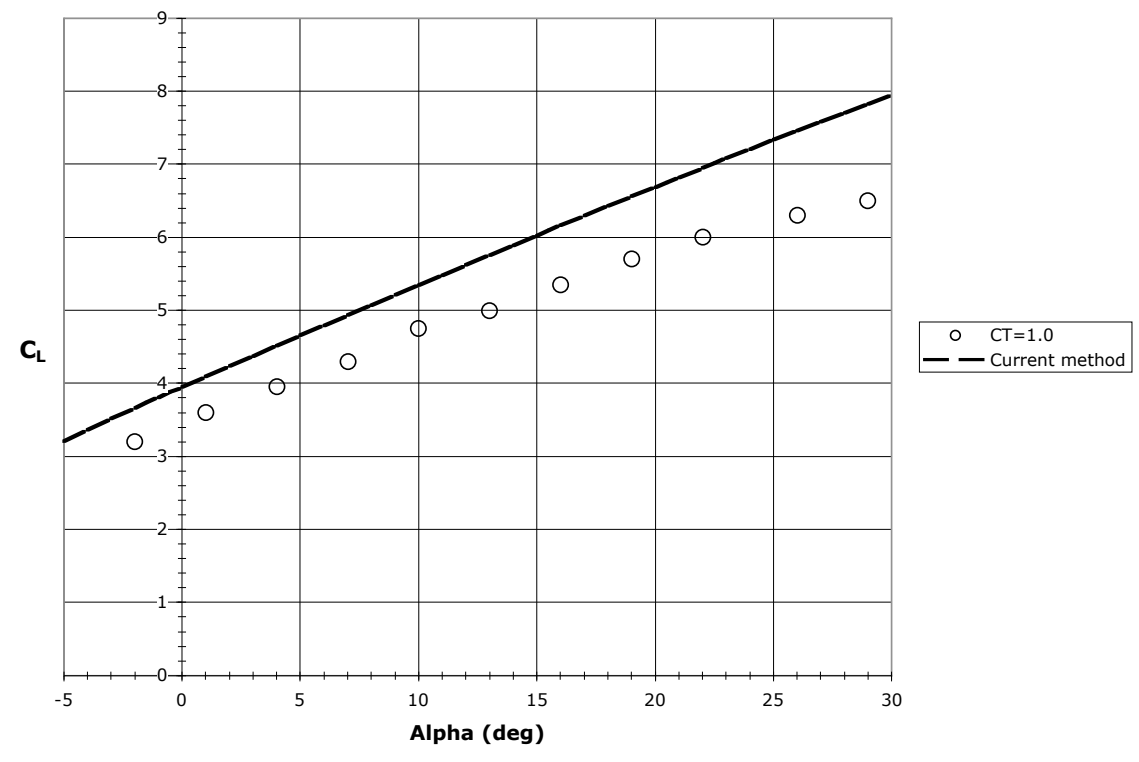

Figure 7-33: Lift coefficient variation with Alpha for landing configuration 


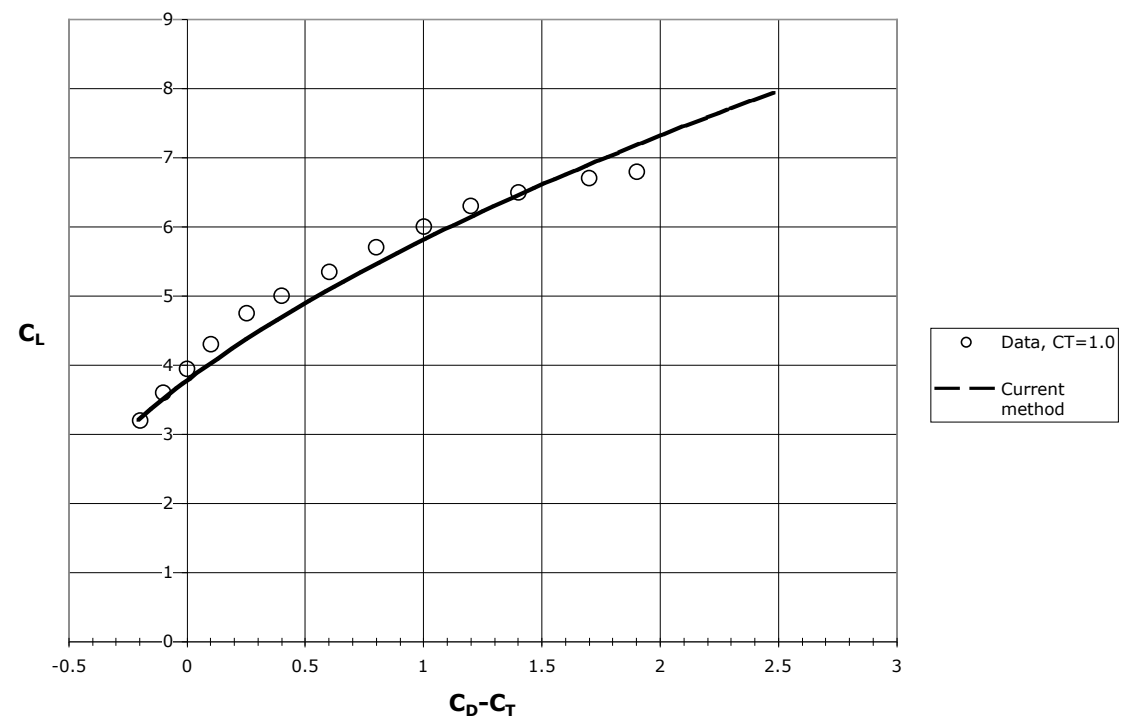

Figure 7-34: Thrust-plus-Drag polar for landing configuration

The lift and drag values for the lower thrust setting are found be in good agreement with experimental data. This fact reinforces the idea that accurate modeling of the propulsion system and flap system are key to obtaining good results.

\section{3 - Brief Comparison with a Non-Linear Vortex Lattice Method}

One of the most successful and comprehensive inviscid panel methods was developed by Mendenhall and Spangler ${ }^{(13)}$. Potential flow models of the lifting surfaces and jet wakes are combined to calculate induced interference on the wings and flaps. The wingflap model is a non-planar, non-linear, vortex lattice method. The multiple chordwise panels allow the curvature of the USB flap to be modeled as discrete planar portions, analogous to the method used in the current work for 2-D. The jet wake is modeled as a rectangular vortex ring distribution, whose strength is determined from engine exit conditions and empirical information on the flow in turbulent wall jets. 
The VLM method can calculate longitudinal effects in incompressible flow. It can handle any kind of wing geometry including dihedral, sweep, twist, and camber. The jet wake model is the key to the success of this method. By basing the jet boundaries and strength on empirical information, the need for complex wake models and iterative solutions for the wake position are eliminated. The superposition of the two potential flow models provides the aerodynamic loading of the wing-flap configuration. To capture the jet reaction force, extra considerations must be made. This force is modeled by distributing extra circulation on each panel beneath the jet such that when acted on by the freestream velocity, it produces a force equal to the reaction force.

Since the non-linear VLM was intended to "stand-alone" in predicting longitudinal aerodynamic characteristics, corrections were applied to account for the effects of leading-edge devices, nacelles, fuselage, and ram drag. Nacelle and fuselage effects were modeled using slender-body theory. No viscous drag corrections are included in this method, meaning that drag consists only of the induced drag, estimated ram drag, and the axial component of the force generated by the wing. Contributions of the estimated ram drag and engine thrust to pitching moment are included in the VLM method.

Comparisons of the VLM against NASA USB reports were presented in Ref. 13. The VLM appears to produce good results for $C_{L}$ and $C_{m}$ for unswept wings at moderate flap deflections and thrust coefficients. Fortunately, some of the comparisons presented in ref 13 overlap with comparisons done in the current work. This allows some direct comparisons between the methods. The report of Ref. 6 was used in both cases, although the flap geometry modeled in the VLM was slightly different. By accounting for the 
different flap geometry (now a constant radius sheet at the trailing edge) and for the loss in turning angle associated with the design, direct comparisons are possible.

Figure 7-35 shows a comparison of VLM, current method, and experimental data for the model of ref 6 (see Figure 7-1) at a flap deflection of 45 degrees:

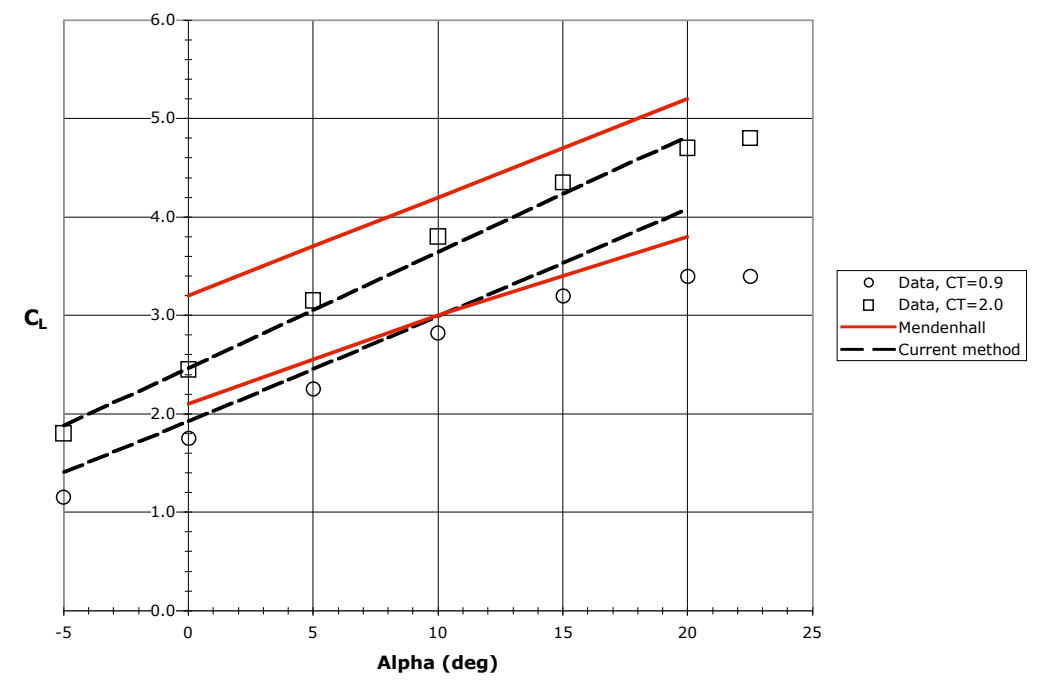

Figure 7-35: $C_{L}$ predictions from VLM and current method for 45 deg deflected radius flap at 2 thrust settings

Note that the VLM cannot account for the low turning angle achieved using a radius flap, a factor that is easily accounted for in the current method. For this configuration, the current method provides equivalent or better estimations of both the lift coefficient (always within $15 \%$ of experiment for unstalled region) and $C_{L \alpha}$. Similar to the proposed method, the VLM, based on potential flow, is incapable of predicting stall. Figure 7-36 shows a comparison of the pitching moments: 


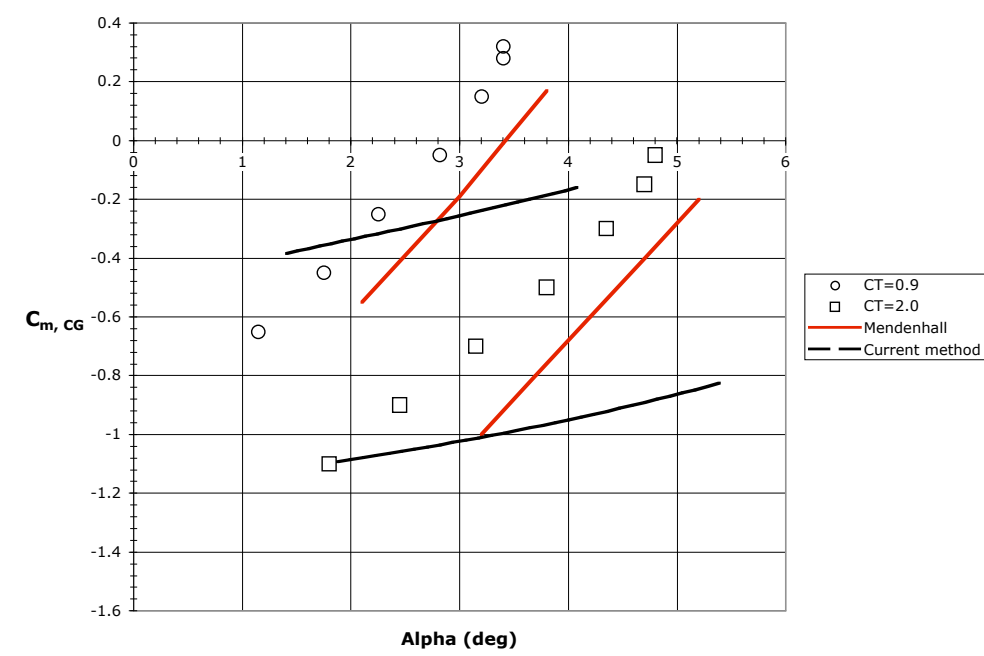

Figure 7-36: Pitching moments about the C.G. for 45 deg flaps at 2 thrust settings Notice that the pitching moment slopes $\left(\mathrm{d} C_{m} / \mathrm{d} C_{L}\right)$ are clearly not predicted well by the current method, while they are accurately predicted by the VLM. However, recall that this is the same aircraft model used in Figure 7-7, where the pitching moments slopes are also not predicted well by the current method. The VLM includes corrections for nacelle, fuselage, and leading-edge slats, while it is not in the scope of the current work to include such corrections. Figure 7-37 shows the drag predictions of the current method with experimental data at 45 degrees flaps.

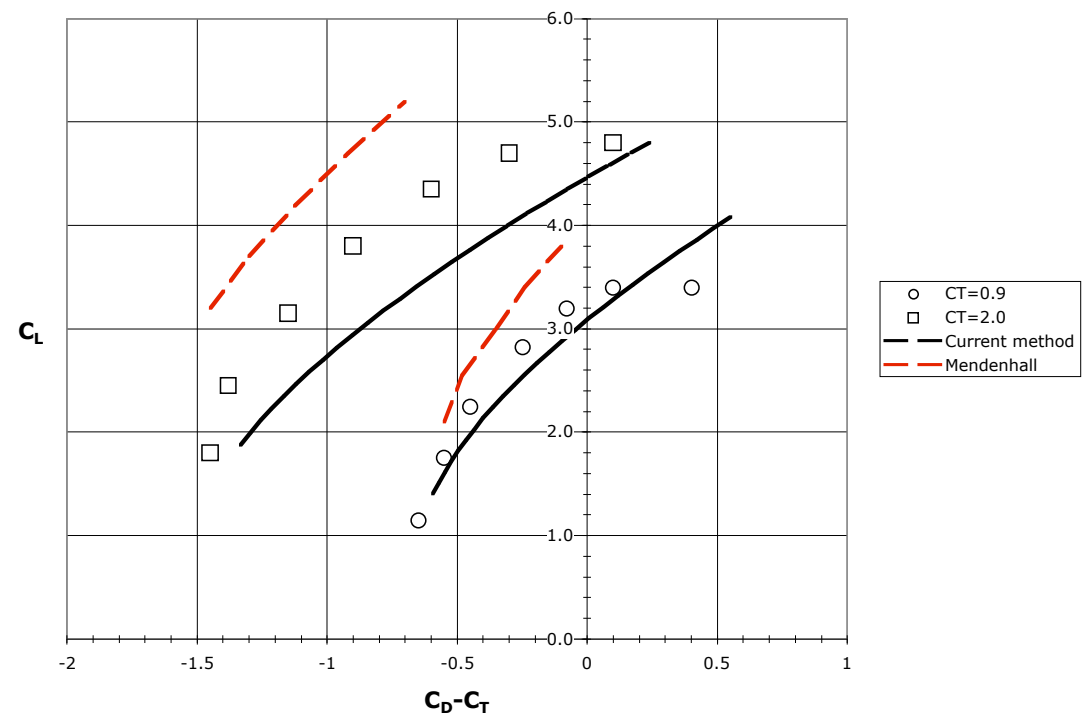

Figure 7-37: Thrust-plus-Drag polars for 45 deg radius flap at 2 thrust settings 
Also presented in Ref. 13 are comparisons of the chordwise pressure variation over a USB flap. These comparisons were made using data from Ref. 8, which was used in the current work to demonstrate the Circular Streamline Theory's effectiveness for USB flaps. It is found the CST provides better estimates of the surface pressures on the flap. Figure 7-38 demonstrates this:

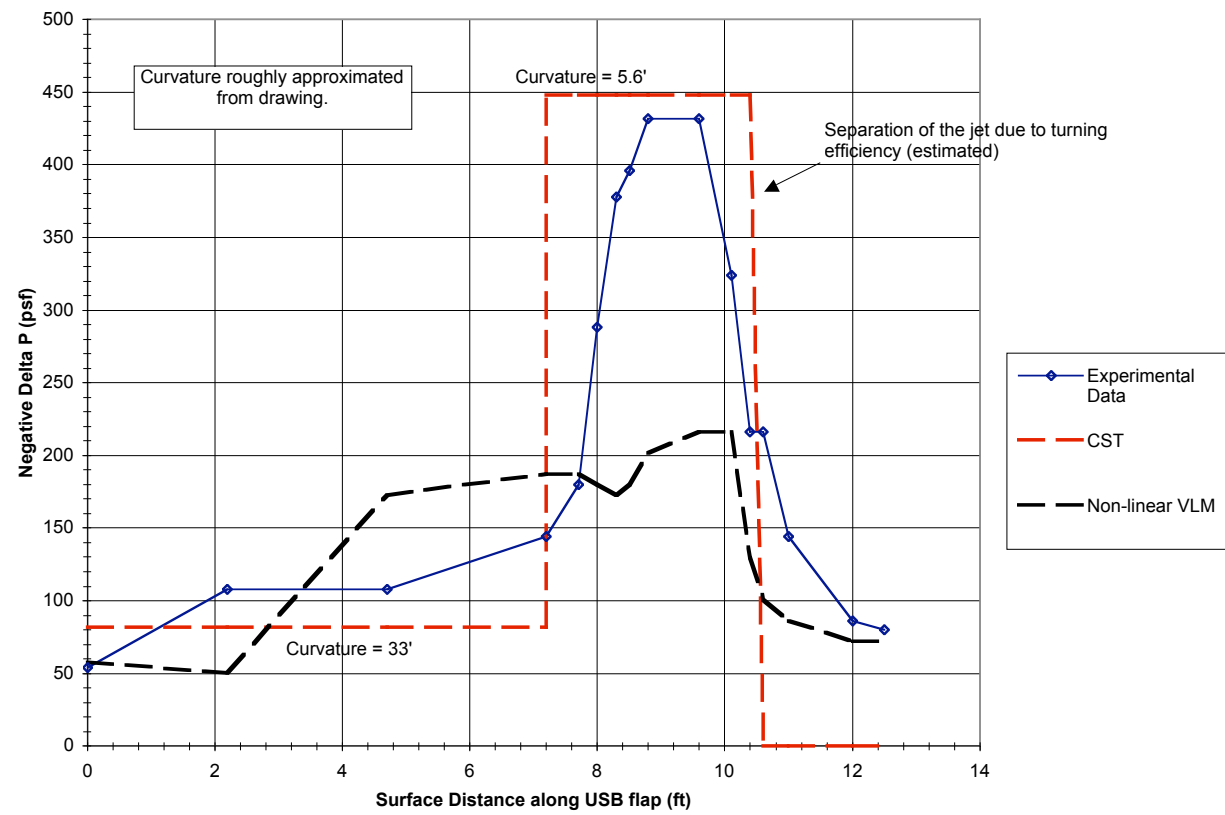

Figure 7-38: Comparison of the non-linear VLM to CST for prediction surface pressures over a USB flap

Note that CST also provides the important capability to provide empirical relations about the turning efficiency of the jet. Thus, the decrease in turning angle of the jet can be modeled well, while VLM can only model losses in thrust due to turning, not the actual angle.

In conclusion, it is shown that the current method compares favorably to a non-linear, vortex lattice method developed for USB. The accuracy of the current method is comparable to the VLM and has the additional benefit of predicting drag, which is crucial 
for USB designs. The weaknesses of the current method are typically shared with the VLM, such as the inability to predict stall and the need for empirical information about the jet. Without an iterative solution for the jet boundaries, the time required to run each method is likely comparable. While the current method would require the generation of new 2-D data for variations in thrust or flap setting, the VLM would require the recalculation of the influence coefficient matrix. Given the prospect for growth of the current method, we believe it is a more appropriate, robust tool for the conceptual design stages of USB aircraft. 


\section{Chapter 8: Conclusions and Recommendations}

The current work has introduced a methodology for the aerodynamic performance predictions of USB aircraft for use in the conceptual and preliminary design phases. We have described the new approach, correlated it with available 2-D data, and presented comparisons of predictions with published USB data. The current approach has been shown to produce good results over a broad range of propulsion system parameters, wing geometries, and flap deflections. In addition, the semi-analytical nature of the methodology will lend itself well to aircraft design programs/optimizers such as ACSYNT. These factors make the current method a useful tool for the design of USB and USB/distributed propulsion aircraft.

It is important to understand the limitations of the proposed methodology. These limitations stem from two main sources: 1). limited empirical relations developed using available 2-D data, and 2). numerical limitations within the modified Weissinger method implementation (high-lift module). The first source was anticipated, and is due to the limited amount of 2-D USB data available that is suitable for creating empirical relations. 
It is believed that the current predictions could be significantly enhanced by a dedicated experimental study of 2-D USB sections with trailing edge devices. Due to lack of 2-D data involving flaps, confidence in the predictions of the current method decrease with increasing flap angle. In addition, the method begins to break down for large blowing coefficients (see the correlations in Chapter 5).

The second limiting factor of the current method is the High-Lift module calculations. Chapter 6 offers a more detailed look at these problems. Breakdown and modification of this code is beyond the scope of the current work, and thus known problems were corrected by post-processing of the output. The usability of the current method would be greatly enhanced by additional work on the High-Lift module specifically directed towards modeling wings with powered-lift wings. Some ideas in this area have already been offered by Chattot ${ }^{(58)}$, and focus primarily on improving the convergence of the solution in cases where the effective angle of attack becomes large.

The current work has been shown to be a useful tool for predicting longitudinal aerodynamic characteristics of low-speed USB wings at low-to-moderate angles of attack. However, the possibilities for future work are abundant. In addition to windtunnel work on 2-D USB characteristics, providing the capability to predict the $\operatorname{stall} / C_{\text {Lmax }}$ of a USB wing would be very useful. Also, since engine-out performance is usually an active constraint on design, estimations of the lateral-directional characteristics are important. This capability would require only minor modifications to the High-Lift module. Future studies in these directions could significantly enhance the current method's relevance to USB aircraft design. 


\section{References}

1. http://www.cnn.com/2004/TRAVEL/07/28/ohare.delays.html

2. http://www.flightdailynews.com/farnborough2004/07 19/airtransport/mega.shtm

3. http://sats.larc.nasa.gov/main.html

4. Hange, Craig, “Trajectory Kinematics of a Simultaneous Non-Interfering Landing Approach, and the Impact on ESTOL Regional Transport Performance and Flight Control”, AIAA Paper 2003-6857, Nov., 2003.

5. Wimpress, John K., Newberry, Conrad F., The YC-14 STOL Prototype: Its Design, Development and Flight Test, AIAA Case Study, AIAA, Reston, 1998.

6. Sleeman, William C. Jr., Hohlweg, William C., "Low-Speed Wind Tunnel Investigation of a Four Engine Upper Surface Blown Model having a Swept Wing and Rectangular and D-Shaped Exhaust Nozzles”, NASA TN D-8061, Dec. 1975.

7. Riddle, D.W., et al, "Powered-Lift takeoff performance characteristics determined from flight test of the Quiet Short-Haul Research aircraft", AIAA 81-2409, AIAA Flight Testing Conference, Las Vegas, NV, Nov. 1981.

8. Sussman, M.B., Reed, J.B., O’Keefe, J.V., and Eldridge, W.M., "USB Environment Assessment Based on YC-14 Flight Test Measurements", AIAA Paper 77-0593, 1977.

9. http://www.globalsecurity.org/military/systems/aircraft/amst-pics.htm.

10. http://ails.arc.nasa.gov/Images/Aeronautics/AC80-0613-3.html

11. Davis, Paul, "Industrial Strength Optimization at Boeing", SIAM News, Vol. 29, No. 1, Jan/Feb, 1996.

12. Roberts, D.W., "A Zonal Method for Modeling Powered-Lift Aircraft Flow Fields”, NASA CR-177521, March, 1989.

13. Mendenhall, M.R., Spangler, S.B., "Calculation of the longitudinal aerodynamic characteristics of upper-surface-blown wing-flap configurations", NASA CR-3004, 1978.

14. Spence, D.A., "The Lift Coefficient of a Thin, Jet-Flapped Wing”, Proceedings of the Royal Soc., Series A, Vol. 238, pp. 46-68, Dec., 1956.

15. Mason, William, “Applied Computational Aerodynamics”, 1997 
16. Smith, A.M.O., "High-Lift Aerodynamics", Journal of Aircraft, Vol. 12, No. 6, pp. 501-530, 1975.

17. Korbacher, G.K., “Aerodynamics of Powered High-Lift Systems”, Annual Review of Fluid Mechanics, 1974.

18. Lan, C.E., Campbell, J.F., “A Wing-Jet Interaction Theory for USB configurations", Journal of Aircraft, Vol. 13, No. 9, 1976, pp. 718-726.

19. Shollenberger, C.A., "Analysis of the Interaction of Jets and Airfoils in Two Dimensions", Journal of Aircraft, Vol. 10, No. 5, 1973, pp. 267-273.

20. Karamcheti, K., Principles of Ideal-Fluid Aerodynamics, Wiley and Sons, 1966.

21. Siestrunck, R., "General Theory of the Jet Flap in Two-Dimensional Flow", Boundary Layer and Flow Control, Ed. G.V. Lachmann, Vol. 1, Pergamon Press, New York, 1961, pp. 342-362.

22. Englar, Robert J., "Circulation Control Pneumatic Aerodynamics: Blown Force and Moment Augmentation and Modification; Past, Present \& Future", AIAA Paper 2000-2541, 2000.

23. Helmbold, H.B., "Theory of the Finite-Span Blowing Wing”, Journal of the Aeronautical Sciences, Vol. 23, May 1957, pp. 339-370.

24. Davenport, F.J., and Hunt, D.N., "Deflection of a Thick Jet by a Convex Surface: A Practical Problem for Powered Lift", AIAA Paper 75-0167, 1975.

25. Schetz, J.A., Boundary Layer Analysis, Prentice Hall, Englewood Cliffs, NJ, 1993

26. Launder,B.E., and Rodi, W., "The Turbulent Wall Jet", Progress in Aerospace Science, Vol. 19, No. 2, 1983.

27. McCormick, Barnes W., Aerodynamics of V/STOL Flight, Dover Publications, New York, 1999.

28. Phelps, Arthur E., et al, "Summary of Low-Speed Aerodynamic Characteristics of Upper Surface Blown Jet-Flap Configurations", Powered Lift Aerodynamics and Acoustics, pp. 63-87, 1976.

29. Henderson, Campbell, and Walters, Marvin, "High-Lift STOL Aerodynamics / Stability and Control Techniques Assessment", Naval Air Development Center Report No. 81261-60, March, 1983.

30. Ryle, D.M. Jr., Braden, J.A., and Gibson, J.S., "Upper Surface Blowing Aerodynamic and Acoustic Characteristics", AIAA Paper 77-0608, 1977. 
31. Narain, J.P., "Viscous-Inviscid Simulation of Upper Surface Blown Configurations", AIAA Paper 84-2200, 1984.

32. Rooney, Eugene C., "Thrust-Drag Accounting Methodology", Thrust and Drag: It's Prediction and Verification, ed. Covert, Eugene E., Progress in Astronautics and Aeronautics, Vol. 98. AIAA, New York, pp. 29-45, 1985.

33. Bowers, Douglas and Tamplin, Gordon, "Throttle-Dependent Forces", Thrust and Drag: It's Prediction and Verification, Ed. Covert, Eugene E., Progress in Astronautics and Aeronautics, Vol. 98. AIAA, New York, pp. 207-279, 1985.

34. Wimpress, John K., "Upper Surface Blowing Technology as applied to the YC-14 Airplane", SAE paper 730916, 1973.

35. May, Fred W., and Bean, George E., "Aerodynamic Design of the Boeing YC-14 Advanced Medium STOL Transport”, AIAA Paper 75-1015, 1975.

36. Hough, G.R., "A Study of the Blown Flap/Jet Flap Analogy", AIAA Paper 79-0119, 1979.

37. Gainer, Thomas G., Yip, Long P., Vogler, Raymond D., "Comparison of Aerodynamic Theory and Experiment for Jet-Flap Wings" Powered-Lift Aerodynamics and Acoustics, NASA SP-405, pp. 103-118, 1976.

38. Spence, D.A., "The Lift on a Thin Aerofoil with a Jet-Augmented Flap", The Aeronautical Quarterly, August 1958, pp 287-299.

39. Davis, W.H. Jr., "Extension of Spence's Jet-Flap Theory for Thin Airfoils to Account for Camber Effects", Grumman Memo EG-ARDYN-79-08, 1979.

40. Roberts, L., "A Theory for Turbulent Curved Wall Jets”, AIAA Paper 87-0004, 1987.

41. Torenbeek, Egbert, Synthesis of Subsonic Airplane Design, Delft University Press, 1981.

42. Roberts, C.R., “An Experimental Investigation of a Powered Wing in TwoDimensions", M.S. Thesis, University of Texas at Arlington, 1984.

43. Pernice, Ciro, “An Experimental Study of a Two-Dimensional Propulsive Wing”, M.S. Thesis, University of Texas at Arlington, 1986.

44. Jeon, C.S., "Experimental Study of a Two-Dimensional Propulsive Wing in a Low-Speed Wind Tunnel”, M.S. Thesis, University of Texas at Arlington, 1990. 
45. Drela, M., "XFOIL: An Analysis and Design System for Low Reynolds Number Airfoils", Low Reynolds Number Aerodynamics, Lecture Notes in Engineering, Vol. 54, Springer-Verlage, June 1989.

46. Weissinger, J., "The Lift Distribution of Swept-Back Wings", NACA TM 1120, 1947.

47. Campbell, George S., "A Finite-Step Method for the Calculation of Span Loadings of Unusual Planforms", NACA RM L50L13, 1951.

48. Blackwell, James A., "A Finite-Step Method for Calculation of Theoretical Load Distributions for Arbitrary Lifting-Surface Arrangements at Subsonic Speeds", NASA TN D-5335, July, 1969.

49. Paris, John Kenneth Floyd, "Advancements in the Design Methodology for Multi-Element High-Lift Systems on Subsonic Civil Transport Aircraft", M.S. Thesis, University of California at Davis, 1996.

50. Vander Kam, Jeremy C., "Reduced-Order High-Fidelity Methodologies for the Design of Civil Transport High-Lift Systems", M.S. Thesis, University of California at Davis, 2000.

51. Roberts, Michael M., "Improvement and Validation of a Reduced-Order HighFidelity Methodology for Civil Transport Aircraft Design", M.S. Thesis, University of California at Davis, 2003.

52. Raymer, Daniel P., Aircraft Design: A Conceptual Approach, AIAA Education Series, 1992.

53. Abbott, Ira H., Von Doenhoff, Albert E., Theory of Wing Sections, Including a Summary of Airfoil Data, Dover Publications, New York, 1959.

54. Smith, Charles C., Phelps, Arthur E., and Copeland, W., "Wind-Tunnel Investigation of a Large-Scale Semispan Model with an Unswept Wing and an Upper Surface Blown Jet Flap", NASA TN D-7526, Feb., 1974.

55. Phelps, Arthur E., and Smith, Charles C., "Wind-Tunnel Investigation of an Upper Surface Blown Jet-Flap Powered-Lift Configuration”, NASA TN D-7399, Dec., 1973.

56. Recktenwald, Gerald, Numerical Methods with MATLAB, Prentice Hall, 2000.

57. Whitten, Perry D., "An Experimental Investigation of a Vectored-Engine-OverWing Powered-Lift Concept”, Technical Report AFFDL-TR-76-92, Dec., 1977. 
58. Chattot, Jean-Jacques, "Analysis and Design of Wings and Wing/Winglet Combinations at Low Speeds", AIAA Paper 2004-220, Jan., 2004. 


\section{Appendix A: Annotated Bibliography}

The purpose of this bibliography is to give the reader a sense of the previous work done on this subject. The body of work that follows was the main base of information for the development presented in this thesis. It will also hopefully serve as a reference tool for anyone seeking a particular topic concerning USB. The sources have been broken into main categories based on the nature of the work done. Within these groups, sources are listed alphabetically by author and then chronologically. The main categories are:

- Powered-Lift Theory

- Experimental Research

- Flight Test Research

- Inviscid/Panel Method Developments

- Computational Fluid Dynamics Developments

- Analytical Developments

This list is certainly not a complete list of work on the subject of Upper Surface Blowing, nor is it limited to sources cited in the report.

\section{A-1: Powered-Lift Theory}

- Henderson, Campbell, and Walters, Marvin, "High-Lift STOL Aerodynamics / Stability and Control Prediction Techniques Assessment", Naval Air Systems Command Report No. 81261-60, pp 45-62, March, 1983.

This is an excellent reference that offers a good description of USB in addition to $\mathrm{IBF}, \mathrm{EBF}, \mathrm{CCW}$, and vectored thrust. It combines the research of many sources into a comprehensive look at USB. The basic aerodynamics are described as being similar to IBF, with good correlation coming from the momentum flux coefficient and deflection angle. A significant performance difference results from changes in flow turning efficiency. This idea is defined, and the primary variables that effect it are discussed. Plots of turning angle and turning efficiency are presented against several geometric parameters.

The high-lift performance of USB configurations is then discussed and plots presented of typical lift and pitching moments. The lateral-directional characteristics are also discussed briefly. A potential flow solution method created by Mendenhall and Spangler (this work is summarized later in this appendix) is presented and the requirements/assumptions discussed. The method is a non-linear vortex lattice method that uses separate systems for the wing and engine wake to help capture the wing-wake interference effects. Similar limitations are faced in this method as in the current method. Complications are still present involving the unknown boundary and shape of the jet. Empirical relations must still be introduced to iteratively solve for these parameters. A comparison with experimental results is presented and shows that the agreement is 
generally similar to that produced in this thesis, though the lift performance is typically over-predicted.

- Helmbold, H.B., "Theory of the Finite-Span Blowing Wing", Journal of the Aeronautical Sciences, Vol. 23, May 1957, pp 339-370.

This paper focuses primarily on a three-dimensional wing with blowing, but also provides a very good discussion on the features of two-dimensional flow. Although jetflap theory was still in its early days when this paper was written, a qualitative knowledge of the flowfield was developing. The physical principle behind blowing is that lift is gained by the jet reaction force, and further lift is gained by "jet-induced" lift. This induced lift is a consequence of the jet sheet modifying the pressure distribution on the airfoil.

For a two-dimensional blown airfoil, a thin jet is blown down at some angle, $\delta$. The pressure discontinuity across the sheet serves to turn the sheet back parallel with the freestream direction. This is the source of our thrust recovery. The resultant of this pressure distribution over the jet can be viewed as a "turning force" which has a lift and drag component. The lift is our reaction lift. By the Kutta-Joukowski theorem, the net horizontal force must be zero, so our drag component is exactly balanced by the leadingedge suction force or leading-edge thrust. Since we've assumed inviscid flow, the lift is taken to be the sum of the reaction force, basic airfoil lift (without blowing), and the induced circulation lift.

The three-dimensional discussion highlights the effect a finite span has on blowing. The primary new effect is the presence of induced drag, which can be significant is these cases. In addition, there are losses of jet thrust and jet-induced lift. The loss of lift is a result of a decrease in curvature of the jet sheet.

- Korbacher, G.K., "Aerodynamics of Powered High-Lift Systems", Annual Review of Fluid Mechanics, 1974.

This paper presents a link between mechanical high-lift systems and powered high-lift systems. It offers useful analogies and a very thorough treatment of powered-lift aircraft. It begins with a broad look at the need for and the factors behind powered-lift designs for STOL or V/STOL aircraft. This includes field length considerations as well as range, noise, and cost factors. The different methods of using power to augment lift are then discussed, beginning naturally with boundary layer control. A good discussion of the jet-flap is presented. The change from BLC to powered-lift is also discussed. The remainder of the paper deals with other powered-lift concepts in turn. 
- Smith, A.M.O., "High-Lift Aerodynamics", Journal of Aircraft, Vol. 12, No. 6, pp 501-530, 1975.

This well-known paper is one of the most fundamental pieces of literature available on mechanical high-lift devices. Although the methodology used in portions of the paper is now outdated, much of the work centers on the theory for high-lift performance. The need for high-lift is presented along with a survey of high-lift devices and their capabilities. The majority of the paper deals with the ability of boundary layers to endure the pressure gradients that mechanical high-lift devices impose. An excellent discussion of multi-element flap systems is included, and shows the reasons why such favorable interference can occur between flap elements.

Only a small portion of the paper is devoted to power-augmented lift. His discussion is geared more towards the use of power to control the boundary layer, but these concepts have a broader application. It is determined that the use of suction to delay separation is only effective when small delays are needed. The use of tangential blowing is preferred. He describes tangential blowing as "making up for the momentum lost in the boundary layer". Tangential blowing leads to discussions of early circulation control airfoils. He notes that a "drag bucket" exists for blown airfoils operating at low blowing coefficient values where the problems of separation are alleviated and have residual thrust from the blowing. Smith also makes an excellent point, as shown by many other researchers, that if a trailing jet is deflected, it will carry a load. Thus it is possible to carry load all the way to the trailing edge of the airfoil and the pressure recovery problem is eliminated.

\section{A-2: Experimental Research}

- F.J. Davenport, D.N. Hunt, "Deflection of a Thick Jet by a Convex Surface: A Practical Problem for Powered Lift" AIAA Paper 75-167, January 20, 1975.

This paper describes work performed by the Boeing Company using a simplified USB configuration to examine factors driving performance. Since the jet turning efficiency of a thick wall jet is of primary importance, this effect was the focus of the study. Simultaneous experimental and analytical programs were conducted. Only 2-D flow was studied for simplicity, and based on the assumption that the planar-flow case probably represents an upper limit to jet turning performance.

A good statement of the fundamental problems faced by USB was included. It was found that the most powerful parameter governing jet turning by a convex surface (Coanda effect) is the ratio of jet depth to the radius of curvature. Three processes were identified that affect USB flow: 1. Mixing layers that reduce flow curvature and thus turning efficiency, 2. BL separation in the adverse pressure gradient between the flap knee and TE, and 3. local acceleration in the jet to supersonic speeds incurring momentum losses.

The experimental program used a small scale model capable of variations in TE radius of curvature and flap angle. (The "flap" consisted of a circular arc) The model 
also allowed for variations in jet thickness. Experiments conclude that the larger the ratio of radius of curvature to jet thickness, the greater the attainable jet deflection becomes. Measurements done of pressure coefficients on the surface show that compressibility effects also become important with increasing freestream dynamic pressure (this implies that USB flows are still quite sensitive to freestream dynamic pressure even though the jet dynamic pressure may be very much higher). Separation of the jet from the flap surface, in essence a decrease of the jet turning efficiency, was seen to occur earlier with increasing freestream dynamic pressure

The analytical model developed was to calculate the 2-D performance and flap surface pressures assuming incompressible, inviscid conditions. The solid wing boundary was represented as a bound vortex sheet, the boundaries of the jet were free vortex sheets, while the jet flow was modeled by a source partially enclosed by the folded back airfoil leading edge. The boundary conditions were no flow across any vortex sheet, and no pressure discontinuity across the free vortex sheets. These sheets were broken up into small line elements of linearly-varying vorticity, and a typical matrix system was solved for the sheet strengths.

The results of the potential flow model demonstrated good agreement for the wind-off condition. An important result was noted that potential flow, as opposed to mixing or other viscous effects, is accountable for much of the jet turning angle deficiencies. The wind-on conditions also agreed reasonably well for low jet Mach numbers. Naturally, as $M_{\text {jet }}$ increases, the potential flow model does not predict the flow well. It was concluded that USB flows must be understood with the presence of external freestream flows, and that the effects of compressibility should be included. Proper USB flap contouring is necessary to ensure good wind-on jet turning.

- Englar, Robert, J., "Investigation into and Application of the High Velocity Circulation Control Wall Jet for High Lift and Drag Generation on STOL Aircraft", AIAA Paper 74-0502, 1974.

The paper examined the basics of trailing edge circulation control to augment airfoil performance. Although it is primarily for a blunt trailing edge with tangential blowing, many of the principles apply to jet-flaps and consequently USB. It was clearly demonstrated that circulation control greatly increases lift and comes with associated increases in drag. The scale of momentum coefficients examined is approximately an order of magnitude lower than typical jet-flap coefficients. This is because without a sharp trailing edge, circulation control is easier to achieve (moving the rear stagnation point is easier). Also, the lift increase comes almost exclusively from circulation lift, since the jet reaction is very small.

The creation of additional profile drag is briefly addressed. As blowing increases, the profile drag is shown to increase. Englar describes this as the result of the jet actually being turned into the lower surface flow, and could also be from the "leading edge" type of flow behavior now present at the trailing edge. Several 2-D investigations were done with an airfoil that could go from a conventional sharp TE at cruise to a full, blunt TE circulation control airfoil using a clever flap design. The intermediate stages basically 
functioned as an internally blown flap. Much study was done on jet detachment, since this will greatly affect the location of the rear stagnation point.

Pressure coefficients around the airfoil were presented, demonstrating the classic "saddle-back" shape with low LE expansion pressures and even lower coanda surface pressures at the TE. Shear stress over the TE demonstrates that the jet very quickly transitions to a turbulent inner region. Results from 3-D testing were also included. For a flap deflection of $180 \mathrm{deg}$, the drag increases in a regular fashion with increasing blowing coefficient. At this condition, there is no thrust recovery due to the larger wake mentioned above. At a deflection of $45 \mathrm{deg}$, where the airfoil is functioning more like an internally blown flap, the drag is shown to decrease as blowing increases. This is because the jet efflux momentum begins to be recovered as thrust. Based on their calculations, up to $70 \%$ of the jet momentum was recovered as thrust. The balance of this momentum can be viewed as lost in jet turning or additional drag penalties with increasing blowing. (Theoretically we recover all the thrust)

- Gainer, Thomas G., Yip, Long P., Vogler, Raymond D., "Comparison of Aerodynamic Theory and Experiment for Jet-Flap Wings”, NASA SP-405, 1978.

Comparisons were made with extensive experiments performed at the Langley V/STOL wind tunnel. Two types of jet-flaps were tested: a pure jet-flap and jet-flap blowing from the knee of a small trailing edge flap (essentially internally blown flaps). Full and partial-span blowing was investigated for wing aspect ratios of 8.0 and 5.5 over a range of momentum coefficients from 0 to 4 . Forces and moments were measured using a strain-gage balance, and pressure data was recorded at 6 spanwise locations across the semispan. For the blown flap, measurements were made at $0,15,30,45$, and 60 degrees. Theoretical predictions were made using a Elementary Vortex Distribution program developed at McDonnell-Douglas, which is a basically an implementation of Spence's jet-flap theory. Some time was spent describing the program, but better programs are currently in use.

For the pure jet-flap, the lift, drag, and pitching moment were found to be in excellent agreement with theoretical predictions regardless of the span of blowing. The lone exception was for a blowing coefficient of zero, where the flow had a good deal of separation. The drag values were shown to be very high, but due primarily to induced drag. Since good agreement was seen with the theory, which assumed $100 \%$ leading edge suction, the model experienced full thrust recovery. The pressure distributions were also presented, and it was seen that the theory underpredicted the pressure coefficients. Thus, the total lift was in good agreement although the components (circulation lift, reaction lift) were different.

For the blown jet-flap, the theory consistently underpredicted the lift, drag, and pitching moment. These results lead to the (now proven correct) conclusion that there is a substantial interaction between the jet exhaust passing over the deflected flap that was not being accounted for by the theory. This effect is clearly seen in looking at the pressure coefficient distributions at a spanwise station with blowing. There is a significant suction area over the flap and it extends forward of the flap knee. 
- Hough, G.R., "A Study of the Blown Flap/Jet Flap Analogy”, AIAA Paper 79-0119, 1979.

A study was done to examine the validity of using jet flap analysis on more advanced propulsive lift systems. The systems studied were the jet-augmented flap (IBF), externally blown flaps (EBF), and upper surface blowing (USB). Analytical predictions were made using a modified lifting line and optimized vortex lattice methods. The "jet flap analogy" suggests that a blown flap system will behave aerodynamically like a jet flap system having the same spanwise distribution of jet momentum.

Experimental data was obtained from separate sources for each of the three lift systems mentioned above.

To carry out the comparison for a particular design, we need to know the trailing edge jet strength, its spanwise distribution, and jet deflection angle in addition to the basic wing geometry. While it is understood that simple jet flap theory cannot accurately predict the complete forces and moments, this study was done to demonstrate that jet flap theory can predict incremental changes in forces and moments due to changes in input parameters. Classic jet flap theory is unable to reflect the true magnitude of the forces and moments because wing and jet thickness effects aren't modeled, nor are the viscous interactions of the wing/jet. The nomenclature used in this paper is very similar to that used in this thesis where $C_{T}$ is the total jet momentum coefficient (basically the gross thrust coefficient), and $C_{\mu}$ is the local momentum coefficient (local 2-D coefficient).

It was found that jet flap theory does indeed approximate the changes in forces and moments well. While the magnitude of these quantities were consistently underpredicted, incremental changes due to changes in wing geometry, angle of attack, blowing coefficient, and engine configuration were predicted fairly well. The predictions were shown to be within $10 \%$ of the expected values for any given configuration.

- Pernice, Ciro., "An Experimental Study of a Two-Dimensional Propulsive Wing”, M.S. Thesis, University of Texas at Arlington, 1986.

This experimental work was done in the low-speed wind tunnel at U.T.A. A small model was built that had a two-dimensional nozzle located at the $70 \%$ chord location on the upper surface. The wing section was based on an NACA 0025 airfoil. Propulsive flows were simulated using compressed air. The primary data taken were pressure measurements around the surface at the mid-span location where the flow was verified to be two-dimensional. Force and moment calculations were attained by integration of these pressures. All coefficients were based upon the chord and a unit span, and thus are sectional coefficients.

Tests were performed at angles of attack of $-5,0,5,10$, and 15 degrees over a range of dynamic pressures $\left(1.0,5.0\right.$, and $\left.10.0 \mathrm{lb} / \mathrm{ft}^{2}\right)$ and jet momentum coefficients $(0.0$ - 3.0). Wake surveys were also obtained, but only used for qualitative analysis. Plots of $C_{l}, C_{m(c / 4)}$, and $C_{d}$ versus momentum coefficient are presented. Variations with angle of attack are also shown. A good discussion on the use of $C_{j}$ (jet momentum coefficient) is presented and it is determined that this factor is not suitable for low blowing cases or for 
cases where the external flow approaches the jet velocity. A more suitable parameter for correlation is the "net momentum coefficient" $\left(C_{f n}\right)$. However, within the range of typical designs, both parameters work well.

The direct entrainment of external fluid by the jet was also studied. It was shown the fluid upstream of the nozzle exit reached higher velocities as the blowing coefficient increased. Minimal discussion on the drag and pitching moment are included, though the pressure drag, $C_{d p}$, was deemed "unreasonably large". A key to this document's usefulness is the inclusion of the data sheets for each point presented. This allows accurate models to be built of the compressed air propulsion system and serves as an excellent check to analysis.

- Phelps III, A.E., Johnson, J.L, and Margason, R.J., "Summary of Low-Speed Aerodynamic Characteristics of Upper-Surface-Blown Jet-Flap Configurations", Powered Lift Aerodynamics and Acoustics, pp. 63-87, 1976.

This paper provided a very good overview of the important characteristics that determine USB performance, stability, and trim. The former is all that is reported here. Of primary importance was the notion that low-speed performance is mainly dependent of the jet turning angle and turning efficiency. This in turn requires the use of proper leading and trailing edge treatment to prevent unwanted flow separation.

Also shown is that USB configurations exhibit a fairly "localized" flow behavior. This means that the effect of the jet flow does not spread much in a spanwise direction. This localized flow field may provide a means through which 2-D sectional data can be used to approximate the overall wing performance. A plot of the spanwise distribution of normal force for a USB configuration indicated that the influence of the exhaust jet on the wing is contained is a region extending approximately 1.75 nozzle widths outboard of the nozzle.

The effects of Reynolds number on USB experimental data led to the conclusion that the performance characteristics are dominated by the exhaust jet rather than the freestream flow (recall that this is low speed flow however and compressibility effects aren't important). This improves the confidence with which small-scale data can be used. It was also observed that, in general, the nozzle geometries that produce good flow turning performance produce poor cruise performance characteristics.

The longitudinal and lateral-directional stability and trim were analyzed with the increased performance of the wing. As expected, with increased lifting characteristics on the wing, the tail volume required for trim increases dramatically. For lateral-directional analysis it was shown that wing swept introduced dynamic instabilities (dutch roll). Lastly, engine-out situations were considered. Naturally, this condition can be very problematic in USB configurations. Boundary Layer Control on the failed engine side was found to make conditions acceptable. (Note that BLC is a reasonable technology, especially since LE blowing could be used to eliminate premature LE separation due to supercirculation) 
- Roberts, Charles R., "An Experimental Investigation of a Powered Wing in Two Dimensions", M.S. Thesis, University of Texas at Arlington, 1984.

This work was the predecessor to the above experimental work by Pernice. As such, the two are quite similar in the design of experiments. A model was created using a NACA 4412 airfoil with compressed air exhausting over the upper surface at approximately $0.6 \mathrm{x} / \mathrm{c}$. Force and moment data were taken over a range of angle of attack from -5 to 15 degrees, and dynamic pressures of 1.0, 5.0, and $10.0 \mathrm{lb} / \mathrm{ft}^{2}$. Based on compressed air restrictions, blowing coefficients of only $0-2.8$ were studied.

The data produced, however, does not seem wholly consistent with later work. The lift coefficients were indicated to be reaching a maximum value within the range of blowing tested. The drag coefficients were also indicated to reach a maximum value. It was suggested by the author that these unexpected patterns were due to interference between the jet and wind tunnel walls.

- Ryle, D.M., Braden Jr., J.A., and Gibson, J.S., "Upper Surface Blowing Aerodynamic and Acoustic Characteristics", AIAA Paper 77-608, AIAA/NASA V/STOL Conference, June 1977.

This paper is basically a summary of exploratory work performed at Lockheed under NASA Langley contracts. The aerodynamic work was largely experimental work on parametric changes in USB configurations. However, some theoretical correlation was also performed. The following advantages over typical high-lift systems were given for USB configurations:

Very competitive High-Lift performance

Adaptive to Hybrid Systems

Mechanical/Structural Simplicity, and

Competitive Acoustical Performance

The following disadvantages were also outlined:

Inherently poor cruise configuration

Sensitivity of wing flow-field at cruise condition

Conflicting high/low speed geometries

Conflicting high lift/ acoustical performance

Extensive experimental data was taken for aerodynamic and acoustic characteristics with changes in geometry. The theoretical modeling was used to support the experimental program and provide clarification of the flow phenomena. The basic premise of the analytical work was to take an existing vortex lattice technique and superimpose nacelle and power effects on a given model. The jet emerging from the nacelle is modeled by an expanding, decreasing strength ring vortex system. Thus, "new" singularity panels can be added to account for power effects. 
- Birckelbaw, L.D., "High Speed Aerodynamics of Upper Surface Blowing Aircraft Configurations" AIAA Paper 92-2611, AIAA Applied Aerodynamics Conference, June 22, 1992.

This paper is focused nearly exclusively on experimental results of tests on a USB aircraft configuration. Particular emphasis is placed on the causes and magnitude of drag penalties of twin and 4-engine USB configurations in high speed flow. These models were based on the Japanese "Aska" Quiet STOL aircraft. The effects of variations in the free stream Mach number, angle of attack, engine power setting, and nacelle spanwise location are presented. Based on these effects, a generic set of design recommendations are presented. No analytical methods were presented.

The results of the experiments found that high speed USB flows exhibit a complex interaction of jet induced and wing transonic flow fields. The engine nacelles created severe drag penalties that increased with freestream Mach number, power setting, and angle of attack. The presence of the nacelles and fuselage/nacelle effects also produced strong upwash over the wing leading edge. (In addition to upwash produced from large jet turning angles) While it was found that a twin-engine configuration developed more lift and less drag for engine speeds below 50,000 rpm, all configurations tested produced prohibitive drag increases at high speeds.

Based upon these findings, the author offers some design recommendations for general USB configurations. First, the twin-engine design is preferable in terms of performance, but may not be preferable in terms of safety (engine out problems). Second, special filleting should be used at wing nacelle intersections to eliminate any flow separation. Third, special consideration should be made to the wing L.E. in the presence of increased upwash near the nacelles. Lastly, the engines should be as far outboard and forward as possible to reduce interference effects with the fuselage. (Note that this must be reconsidered for a twin-engine configuration)

\section{A-3: Flight Test Research}

- Sussman, M.B., Reed, J.B., O’Keefe, J.V., and Eldridge, W.M. "USB Environment Assesment based on YC-14 Flight Test Measurements”, AIAA Paper 77-593, 1977.

This paper was a summary of flight tests and static rig tests of the environment around the USB flap of the Boeing YC-14. Static pressures, surface temperatures, surface fluctuations, and vibration levels were measured. This project was done by Boeing in conjunction with a NASA initiative. Wing and USB static pressure ports and thermocouples, along with high-frequency acoustic transducers were installed on the fuselage, engine nacelle, USB flaps, and upper suface behind the nozzle.

The results of testing for the upper surface/flap pressure measurements were presented first. For a fixed USB flap setting, increasing thrust naturally generates increasingly negative pressures on the main flap section. The aft flap section changes were smaller indicating a lesser contribution to turning effectiveness from this flap. For a fixed power setting, increasing USB flap deflection induces larger suctions on the main 
flap. The aft flap suctions increase until approximately 40 degrees where pressures then begin to decrease. Also, as expected, the turning efficiency is somewhat reduces in the presence of a freestream. This is due to the stronger adverse pressure gradient that develops on the aft side of the USB flap.

The temperature measurement were made in low-speed/takeoff and landing conditions where the USB system in full use. While the results may be very dependent on the particular configuration, the results were still reasonable. The main flap experienced temperatures around 400 degrees F, with the aft flap around $250 \mathrm{deg}$. F. The temperatures measured on the body near the flap never exceeded $145 \mathrm{deg}$. F. Also, the flight temperatures were generally 50 to 100 degrees lower than these static rig results. Acoustic environment results were also presented but are not summarized here. It was concluded that the flight data and wind tunnel data agree fairly well, and that USB sonic and temperature data were less severe in flight conditions than predicted by static tests.

- Wimpress, J.K., and Newberry, C.F., "The YC-14 STOL Prototype: It's Design, Development, and Flight Test", AIAA Case Study, Reston, 1998.

The document is an extensive look at Boeing's YC-14 STOL prototype, from the RFP to flight testing. Although it was originally written some time ago, it has been updated recently. Because the high-lift capabilities of the YC-14 were based around the concept of USB, this document contains some interesting information on the background of the idea and its implementation. Only material relating to this is summarized here.

The Boeing company first became interested in the USB concept in the late $60 \mathrm{~s}$ when the design implications of a jet's tendency to adhere to an adjacent physical surface were first being realized. Their wind tunnel test program verified previous results and also showed far greater thrust recovery than externally blown flaps. It was also seen that L.E. BLC must be used to prevent premature separation and adequate control in an engine-out situation. Some of the test data taken was reported in this document and they seem to agree with agree with conclusions reached in other papers - basically that effective jet turning can be accomplished with appropriate design and that small scale USB testing models full scale USB configurations very well.

The nozzle design was a key feature in the success of the YC-14 design. The nozzle outer contours were shaped to prevent high drag under cruise conditions. In addition, a small door was placed on the outboard side of the nozzle that would open during high-lift operation. This door allowed the engine exhaust jet to spread further outboard over the USB flap. It also increased the effective exit area. To increase jet turning performance, retractable vortex generators were placed near the T.E. of the basic wing. These generators came into use when flap deflections became large $\left(>30^{\circ}\right)$ and basically increased mixing of the jet flow with the local boundary layer and delayed jet separation until farther downstream.

An extensive boundary layer control system was also designed. Cross-ducting permitted the continued use of BLC even in engine out cases. Interestingly, precautions had to be made against any ruptures in this ducting, as it was shown that cabin temperatures would rise to fatal levels very quickly. The boundary layer control system, coupled with variable camber Krueger flaps, helped prevent premature flow separation 
near the leading edge. Although this aircraft never reached production, it is still one of the only aircraft in existence to fully utilize upper surface blowing technology effectively.

\section{A-4: Inviscid/Panel Method Developments}

- Lan, C.E., and Campbell, J.F., “A Wing-Jet Interaction Theory for USB

Configurations", Journal of Aircraft, Volume 13, No. 9, pp. 718-726.

This paper develops a linear, inviscid, subsonic compressible flow theory to treat the interaction between a wing and an upper surface thick jet. Upper Surface Blowing produces additional lift through this interaction, and through the jet entrainment producing the Coanda effect. Theoretically, an exact solution could be found from analysis of a three-dimensional turbulent wall jet in a wing flowfield. The method presented is for a wing in freestream Mach, $M_{o}$, with a thick jet of Mach number, $M_{j}$, on or above the wing surface. It is assumed that the flow fields (inside and outside of the jet) are governed by the Prandtl-Glauert equation.

The boundary conditions imposed were that the jet surface be a stream surface with static pressure continuous across it, and that the flow be tangential to the wing surface. The wing is represented by a vortex distribution, while the jet surface is modeled as 2 coincident vortex sheets (one to account for perturbations in the outer flow, one for the jet flow) Using quasi-VLM, additional vortex strengths induced on the wing can be determined. Once the total vortex distribution on the wing is known, expressions were determined for the aerodynamic forces and moments.

Some comparisons were made with experimental data (much more experimental data would be available at this point) and showed good results, particularly at small flap/jet deflections. A comparison was made of predictions using thin jet flap theory, and it was shown that this theory is inadequate for predicting the aerodynamic characteristics of USB configurations.

** A FORTRAN code was written for this method and is available in NASA report TM$\mathrm{X}-73987$

- Mendenhall, M.R., and Spangler, S.B., "Calculation of the Longitudinal Aerodynamic Characteristics of Upper-Surface-Blown Wing-Flap Configurations”, NASA CR-3004, 1978.

This contractor report from NASA outlines the development of a non-linear vortex lattice method for the calculation of USB performance. Subsequently, the code itself is printed in CR-3005. This methodology is the most useful and accurate of the inviscid methods investigated in the course of this work. It incorporates two potential flow models for the wing and jet. The wing can have an arbitrary planform and the jet is assumed to be rectangular and have a fixed aspect ratio. It provides estimates of 
chordwise pressure distributions, spanload distributions, total longitudinal coefficients over a wide range of angles of attack and thrust settings.

The wing planform is unremarkable with the exception of the modeling of a constant radius trailing edge flap. The semi-infinite legs of the trailing vortices are assumed to go to infinity in the plane of the last chordwise surface. The jet wake model is comprised of vortex rings. The strength of the rings is determined through empirical relations concerning the jet spreading rates. The jet exit velocity is determined through isentropic relations and the jet wake is assumed to maintain a constant aspect ratio. The wake model provides an interference effect on the wing that mimics the physical behavior of USB.

Some corrections and extensions were created to provide estimations for the whole aircraft. Fuselage and nacelle effects were modeled using slender body theory to calculate lift forces and centers of pressure. Ram drag was also accounted for in the code. Predictions were made using this methodology for several NASA experimental USB reports. These comparisons indicate that this method performs very well at low angles of attack (unstalled regions) and at moderate thrust coefficients. The spanload distributions were predicted well, but the chordwise distributions were not. Drag was typically under-estimated due to the lack of viscous effects in the calculations.

- Narian, J.P., "Viscous-Inviscid Simulation of Upper Surface Blown Configurations", AIAA Paper 84-2200, AIAA Applied Aerodynamics Conference, Aug. 21, 1984.

This study investigates USB by using a combination of inviscid panel methods and integral methods for viscous wall jets on curved surfaces in non-uniform flow. This study is a step in the direction suggested by Lan (see above). Study suggests that viscous-inviscid methods involving panel methods and parabolized N-S solutions could be very effective, but were computationally expensive. This method uses a more approximate method where the inviscid flow field is solved by panel methods, then the entrainment velocity and plume boundaries of the jet are calculated using a 3-dimensional integral solution. Aft of the trailing edge, a "jet in cross-flow" theory is used.

The following process was used to obtain details on the jet behavior and pressure distributions on the airfoil sections:

-The wing-body-nacelle configuration is analyzed via panel methods for the unpowered (flow-through) case

-The pressure distribution at the nacelle centerline is used to calculate the nonuniform external velocities for the wall jet development

-Integral analysis is used to determine the jet boundaries and jet entrainment velocities at several panel stations

-The vorticity associated with the jet is imposed on the wall jet panels as a doublet gradient

-The jet boundaries, prescribed entrainment velocities, and prescribed doublet gradient are used to create new panels to simulate the effects of jet blowing within the panel method. 
Results on jet decay were then presented, and it was noted that convex curvature of the wall accelerates the decay of the wall jet, which results in rapid expansion of the jet boundaries. A USB model was tested experimentally and compared to theoretical results with favorable agreement. The lift coefficients predicted at various nozzle pressure ratios agreed very well with experimental data. Pressure coefficient distributions were also calculated.

- Shollenberger, C.A., "Analysis of the Interaction of Jets and Airfoils in Two Dimensions”, Journal of Aircraft, Vol. 10, No. 5, pp. 267-273, 1973

A solution for the flow of a jet near one or more airfoils was developed. Flow is assumed to be inviscid, incompressible, and two-dimensional. The problem is governed by Laplace's equation with a no-flow boundary condition enforced on the wing, as well as across the jet. In addition, the jet involves a pressure boundary condition where the static pressure is constant across the jet boundary while velocity can be discontinuous. The velocity discontinuity is related to the total pressure difference between the jet and external streams. The primary difficultly involved defining a jet surface on which to apply these conditions.

The method of solution uses "flat-plate" airfoils modeled using vortex sheets. The jet boundaries can be modeled by vortex sheets whose strengths are related to the local mean velocity. The singularities of the airfoil are easy to determine since we set the airfoil position. The downstream jet is also easy to fix since its direction must asymptotically approach the freestream direction. The near-field jet location can be found along with the singularity strengths in an iterative process. Some convergence issues were discussed, including under-relaxation to improve convergence. Viscous effects, which are of course ignored in an inviscid formulation, are described as confined to the conventional boundary layer, and entrainment of the external fluid. The entrainment could be empirically modeled by altering the boundary condition imposed on the jet.

Some computations using this method were presented. The first is similar to an EBF system with a lower jet impinging on the lower airfoil surface and aft deflected flap. It was found that though a majority of the load was induced on the aft flap (small deflected flat plate), a significant load was induced on the main element even though it was at zero angle of attack. The vertical position of a jet relative to an airfoil was also investigated. While a peak load was experienced when the airfoil was completely immersed, significant loadings were induced as the jet neared the upper or lower surfaces. 


\title{
A-5: Computational Fluid Dynamics Developments
}

\author{
- Bickelbaw, L., Atta, E., and Ragab, S., "Euler Solutions for Aircraft Configurations \\ Employing Upper Surface Blowing”, Journal of Aircraft, Vol 24, No 3 pp 188-194
}

This paper outlines a zonal method for computing the flowfield around aircraft with USB. This method is based on zonal grid generation coupled with an Euler flow solver. A significant portion of this paper dealt with the grid generation and mating. The flow solving technique was based on the Euler equations with a finite-volume timestepping Runge-Kutta scheme. Artificial viscosity was added to the process through "dissipation constants", and boundary conditions were typical for sub/supersonic cases.

Computations were initially run for 2-D cases (with 2 zones which represented the exhaust region and the rest of the flowfield). The process did capture the pressure peaks induced by the jet and showed the expected variation with jet pressure ratio. The results for 3-D were compared with experimental data and less complicated panel methods. The Euler solutions performed very well with only some problems where nacelle effects were dominant and at the T.E. where viscous effects were dominant. This method may prove very effective but is not extremely useful in understanding what factors influence the flowfield changes.

- Roberts, D.W. “A Zonal Method for Modeling Powered-Lift Aircraft Flow Fields", NASA-CR-177521, March 1989.

This paper is similar to the methods described by Narain (see above). The method described is a zonal method based on the coupling of a 3-D Navier Stokes code to a potential code. The coupling allows the appropriate modeling of the viscous/inviscid interactions that dominate powered-lift configurations. Zonal methods involve dividing the flow domain into a network of zones in which the simplest, appropriate flow analysis is used. Of key importance is the boundary conditions between the different zones. Since the potential flow is a subset of the N-S equations, the N-S zone must extend well into the region where viscous phenomena aren't present. However, making the N-S zone too large can greatly increase computation time.

The panel method basically views the viscous zones as extensions of the configuration and are influenced through blockage effects and entrainment. Mass consveration is imposed going from N-S to panel zones. Similarly, the N-S method is influenced by the potential regions. The total pressure, temperature, and direction cosines of velocity are passed from the potential code to the $\mathrm{N}-\mathrm{S}$ code. This paper goes into some detail on the modifications made to the codes, and comparisons of results to wind tunnel data. Although it appears that the correlation is very good, those results will not be summarized here.

This method, while very powerful and accurate, may be too computationally intensive for conceptual-level codes. The underlying principles of zonal methods may, however, be a very good way to approach USB configuration analysis. 


\section{A-6: Analytical Developments}

- Davis, W.H. Jr., "Extension of Spence's Jet-Flap Theory for Thin Airfoils to Account for Camber Effects", Memo EG-ARDYN-79-08, Grumman Aircraft, 1979.

A parallel method was used to extend the analytical work of Spence (see below) to airfoil sections with parabolic camber. Similar-order curve fits were also presented. Since jetflap theory is linear, this work "plugs-in" directly with the classical development. As with Spence, small disturbance theory was used to develop integro-differential equations. These were solved to form an additional term contributing the lift coefficient as:

$$
\Delta C_{l}=4 \pi\left(1+C_{o}\right) \gamma
$$

where gamma is the maximum camber and $C_{o}$ is an empirically defined constant dependent on the blowing coefficient.

- Spence, D.A., "The Lift Coefficient of a Thin, Jet-Flapped Wing”, Proc. of the Royal Society of London, Series A, Vol. 238, Issue 1212, pp. 46-68, 1956.

This paper is widely recognized as one of the most important early works done on powered lift. Spence recognized that lift is supplied not only by the reaction to the vertical momentum of the jet, but also by the modification of the surface pressures due to the changes in the flowfield induced by the jet. The correlating parameter was taken to be the momentum coefficient. It should be noted that the equation for this coefficient (eq 34) does not explicitly show the chord length in the denominator since it is unity for this paper. Since these are 2-D coefficients, it is also implied that these quantities are "per unit span”.

An analytical solution is given for the inviscid, incompressible flow past a thin, two-dimensional airfoil at reasonable angle of attack for which a thin jet emerges at the trailing edge at some deflection angle, $\tau$. The flow is also assumed irrotational, so potential flow methods are used. The airfoil and jet are modeled using distributed singularities and an "integro-differential" equation is obtained that is solved by clever used of Fourier series. The basic equation is presented as:

$$
C_{l}=2 \pi \alpha+4 \pi B_{o} \alpha+4 \pi A_{o} \tau
$$

where $A_{o}$ and $B_{o}$ are coefficients dependent on the momentum coefficient. A close interpolation to these coefficients is given for momentum coefficients between 0 and 10 .

Similar data was given for the pitching moment, but was not developed into the convenient form shown above. Pulling this data together was one purpose of this thesis. Some comparison with experimental data was performed and demonstrated the value of the theory. In the years since, the value of the theory (and the theory's limitations) have been shown more completely. 


\section{Appendix B: Sample of Data Generation for 2-D Method}

The purpose of this appendix is to provide an example of the typical process for generating/collecting the necessary input data for the 2-D methodology. The full 2-D equations can be seen in equations (4-38) through (4-40) and clearly involve terms that require a degree of knowledge about the configuration being studied. Further knowledge about the wing itself is needed to carry out 3-D performance calculations. For the purposes of this appendix, the process of obtaining or calculating the necessary input information for the Boeing YC-14 is presented.

\section{B-1: Overview}

In general, conceptual level analysis of Upper Surface Blown wing performance will require information in 5 categories:

1. Planform Data - wing shape and size, engine locations, spanwise locations of trailing/leading edge devices.

2. Flap Geometry - flap chord ratio, flap curvature and extension, flap deflection angle, trailing edge angle of base airfoil

3. Approximate Nozzle Geometry - Nozzle height and width (or nozzle exit area), chordwise location of nozzle exit

4. Propulsion Data - aicraft total thrust coefficient, nozzle exit conditions

5. Flight State Data - Mach number, altitude

This information is generally readily available for a conceptual design, or reasonable estimates can be made. Flap geometry and flap kinematics is the most detailed category by necessity, since this data will have a large effect on the performance. Once the data of the five categories has been calculated, estimated, or looked-up, the 2-D and 3-D calculation methods described in this work are straight forward to use and obtain good results.

\section{B-2: Planform Data}

Planform data is necessary primarily for the 3-D Weissinger calculations, but some key pieces of information are also required for the 2-D approximations. In the case of the YC-14, a significant amount of aircraft layout information was available. Technical drawings of the aircraft are available in the case study by Wimpress and Newberry ${ }^{(5)}$. In most design cases, the planform information will not be as definite, and some quantities should be judiciously approximated.

Figure B-1 shows a sketch of the YC-14 wing planform: 


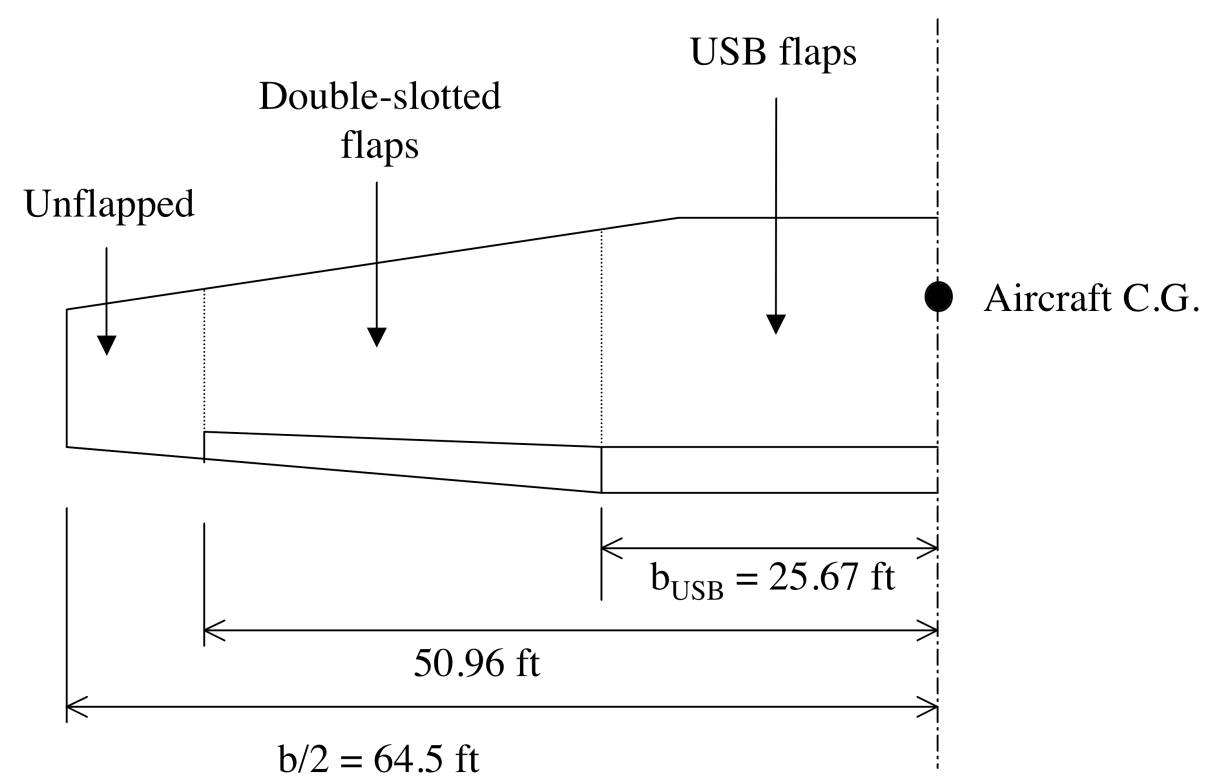

Figure B-1: Sketch of the YC-14 wing planform

From the drawings and reference we gather the following necessary information:

- Planform shape, including taper, twist, and incidence.

- Wing area, $S=1762.4 \mathrm{ft}^{2}$

- Engines located at $\eta=0.254$

- Airfoil section type $=$ supercritical

- Airfoil section camber = maximum displacement of camber line / chord (assumed zero for the YC-14 calculations, airfoil camber plays only a small role)

- $\quad$ Root thickness $=0.16 \mathrm{t} / \mathrm{c}$

- Trailing edge devices include inboard USB flaps up to $\eta=0.398$, then multielement flaps midspan to $\eta=0.790$, no high-lift devices outboard

- Average chord over blown sections $=17.5 \mathrm{ft}$

- Span of blowing (determined in this case by the physical extent of the USB flaps, but in a design case should be approximated in light of nozzle spreading and number of engines per wing), $b_{U S B}=25.667 \mathrm{ft}$

\section{B-3: Flap Geometry}

As mentioned above, these quantities can be the hardest to determine and may be significant players in the design of a USB aircraft. In the case of the YC-14, some data was given in the detail drawings that demonstrate the extension and curvature of the flaps at various deflections. This allowed for accurate modeling of the flap system, but such data will generally not be available. It is assumed that the USB flap system is a nested configuration that deploys to a given deflection maintaining a continuous surface as demonstrated in Figure B-2: 

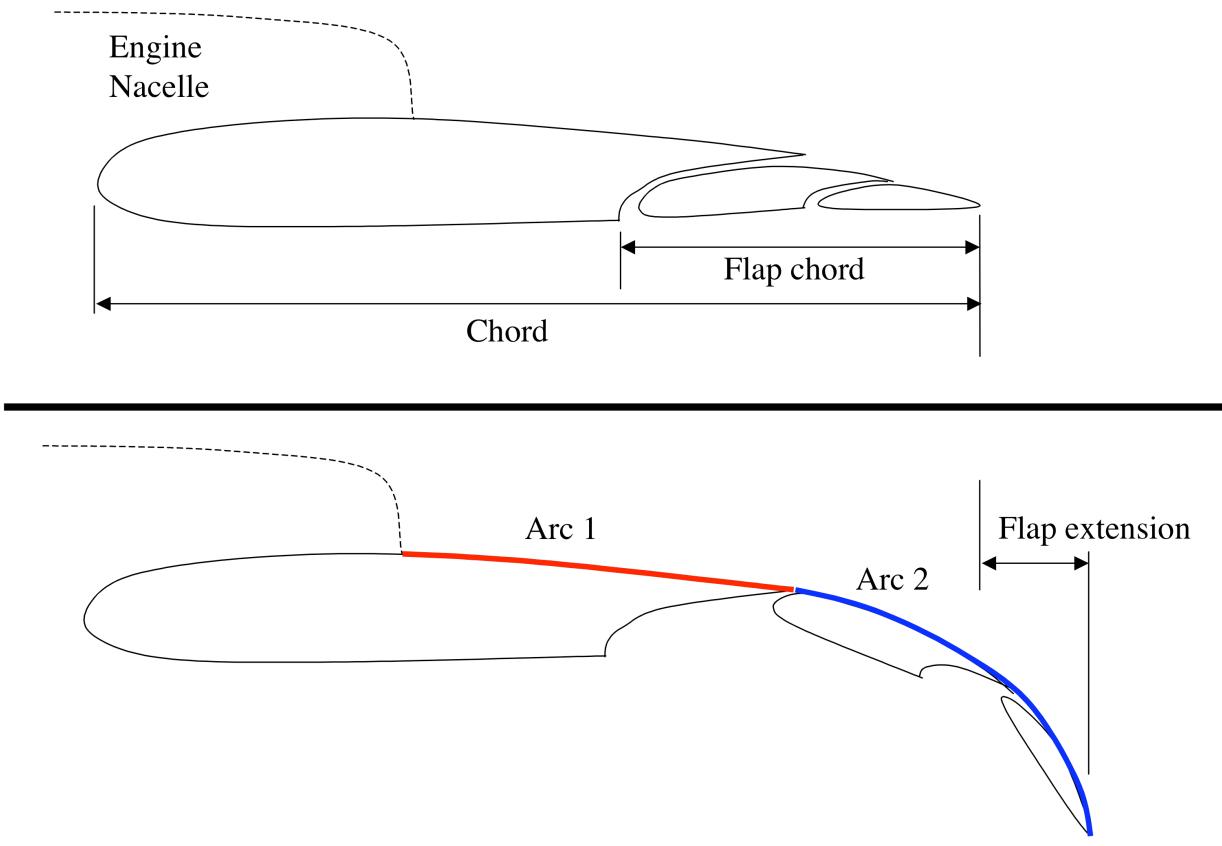

Figure B-2: Typical USB flap configuration

The minimal acceptable information consists of:

- Flap chord ratio, $E=0.30$

- Flap parameter, $\chi=2 \sin ^{-1}(\sqrt{E})$

- $\quad$ Curvature of upper surface $($ Arc 1) $=33 \mathrm{ft}$.

- Curvature of USB flap at given deflection (Arc 2) $=5.6 \mathrm{ft}$.

- Trailing edge angle of base airfoil (XFOIL is very useful for this information), $\theta_{T E}=0.24 \mathrm{rad}$

- Given USB flap deflection, $20^{\circ}$ at takeoff, $60^{\circ}$ at landing

- Total deflection of jet, $\delta_{f}=$ input flap angle $+\theta_{T E}$ (the jet leaves the trailing edge tangent to the surface)

\section{B-4: Nozzle Geometry}

The exact geometry of the nozzle was well known in this case. In a design case, the geometry of the nozzle is typically a detail design feature requiring satisfaction of several disparate requirements. To ensure a good jet shape, the exit area of the nozzles is typically rectangular or nearly rectangular. Thus, this work assumes a rectangular nozzle shape. Input consists of the following variables:

- $\quad$ Nozzle height, $h=2.0 \mathrm{ft}$

- Initial jet thickness, $t=h$ ( $t$ will be assumed equal to $h$ over the full flap)

- $\quad$ Nozzle exit area, $A_{\text {exit }}=17.2 \mathrm{ft}^{2}$

- Chordwise location of nozzle exit, $x / c=0.30$ 


\section{B-5: Propulsion Data}

For the case of the YC-14, it was known that CF6-50D engines were used. Appropriate data sheets contained the gross thrust, exit velocity, and mass flow rates for these engines. Due to the use of Circular Streamline Theory, the exit conditions are vital to the 2-D calculations. In a design case, where a rubber engine approach may be used, typically only the gross thrust may be specified. In this case, isentropic relations with suffice to provide the exit conditions as follows. From the thrust:

$$
T_{\text {Net, Design Condition }} \cong \dot{m}\left(V_{\text {exit }}-U_{\infty}\right)
$$

where the mass flow rate is:

$$
\dot{m}=\rho_{\text {jet }} A_{\text {exit }} V_{\text {exit }}
$$

More exact relations involving nozzle pressure ratios are preferable to these basic relations. The most critical propulsion parameter we must determine is the momentum flux coefficient or blowing coefficient for the 2-D calculations. This parameter assumes that the engine operates at the design condition and is easy to find once the exit conditions are known:

$$
C_{\mu}=C_{j}=\frac{\dot{m} V_{e x i t}}{q_{\infty} c b_{U S B}}
$$

where the dynamic pressure comes from flight-state data and $c$ represents the average chord over the blown section. For the YC-14, given total thrust coefficients of 1.0 and 2.0, the relatively small blowing span produces 2-D blowing coefficients of 1.9 and 3.9, respectively.

\section{B-6: Flight State Data}

This data determines the freestream conditions the aircraft experiences. These may vary significantly between points of interest, such as takeoff, landing, cruise, or maneuver points.

- $\quad$ Altitude/Density, $\rho_{\infty}=0.002377 \mathrm{sl} / \mathrm{ft}^{3}$

- $\quad$ Mach Number, $M_{\infty}=0.1$ 


\section{Vita}

Ernest Brock Keen was born on August 4, 1979 in Lebanon, Virginia. He grew up there with his parents, Jack and Sheila, younger brother, Jordan, and older sister, Stephanie. After high school graduation, he moved to Blacksburg, Virginia to attend Virginia Tech in Aerospace Engineering. As part of his undergraduate curriculum he was a part of the Honors Program and the Co-operative Education Program. He spent one semester with Allied Signal Aerospace Repair \& Overhaul (now Honeywell) in Greer, South Carolina. Another semester was spent working as a test engineer for Pratt \& Whitney, Large Military Engines in Palm Beach Gardens, Florida. During his time at Pratt \& Whitney, he was a member of the Boeing test team for the CTOL qualification engine for the X-32. He graduated Summa Cum Laude in May of 2002. After graduating, he returned home for one year to assist in the development of his father's land surveying business. In May of 2003 he returned to Virginia Tech for his M.S. degree with Dr. William Mason. During his time pursuing his Master's degree, he worked with AVID, LLC in Blacksburg, Virginia in engineering studies for NASA and Honeywell. 